\title{
Identification of resistance sources and characterization of resistance factors in Brassica species to Verticillium longisporum
}

\author{
Identifizierung von Resistenzquellen und Charakterisierung von Resistenzfaktoren \\ in Brassica-Arten gegenüber Verticillium longisporum
}

\author{
Dissertation \\ zur Erlangung des Doktorgrades \\ der Fakultät für Agrarwissenschaften \\ der Georg-August-Universität Göttingen
}

\author{
vorgelegt von \\ Christina Eynck \\ geboren in Bad Karlshafen
}

Göttingen, im Dezember 2007 
D 7

1. Referent : Prof. Dr. Andreas von Tiedemann

2. Referent : Prof. Dr. Heiko Becker

Tag der mündlichen Prüfung : 31. Januar 2008 
meinen Eltern Jürgen und Ingrid Eynck gewidmet 


\section{Contents}

Chapter 1: Introduction ............................................................................... 1

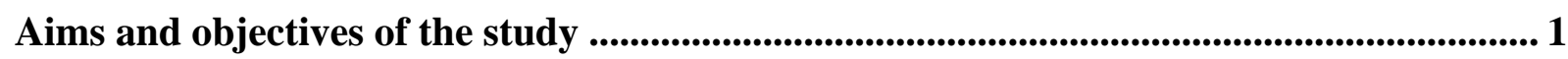

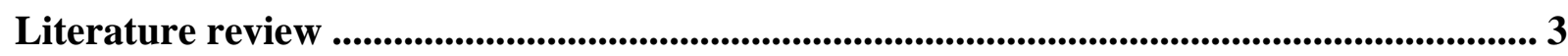

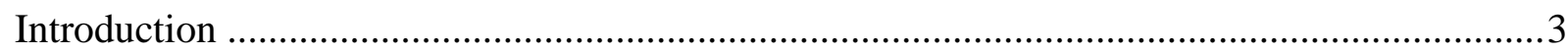

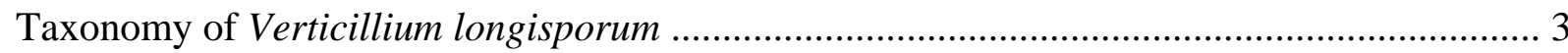

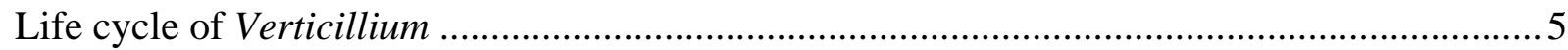

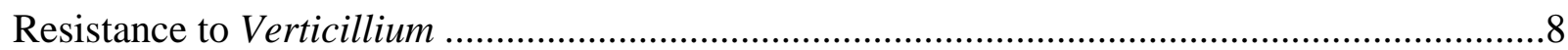

\section{Chapter 2: Identification of Brassica accessions with enhanced resistance to} Verticillium longisporum under controlled and field conditions

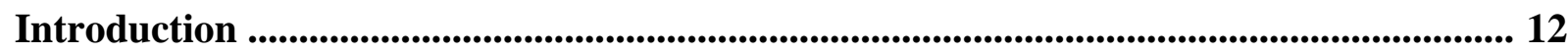

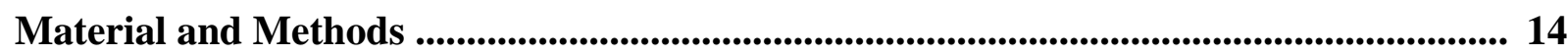

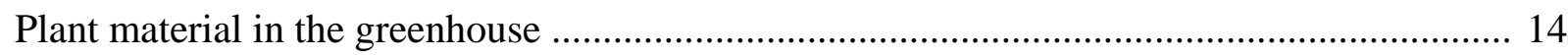

Inoculation and screening procedure in the greenhouse............................................... 15

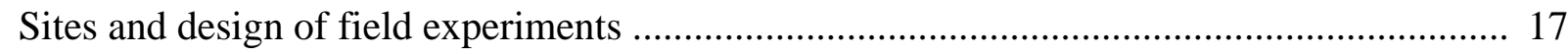

Visual scoring of $V$. longisporum disease symptoms in the field ...................................... 19

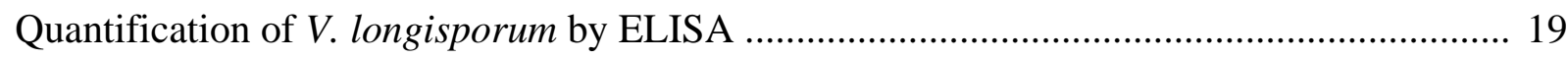

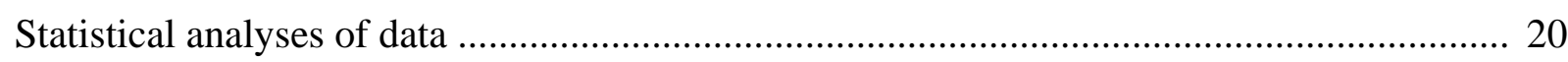

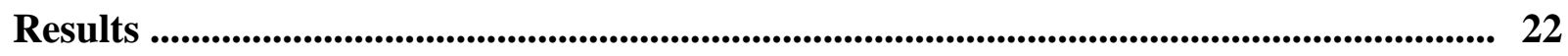

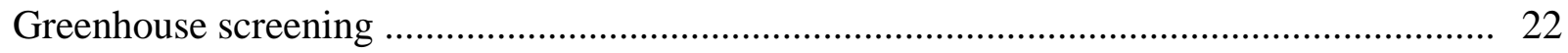

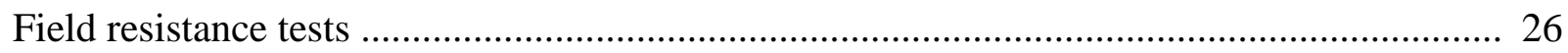

Comparison of results from resistance tests in the greenhouse and in the field .................... 28

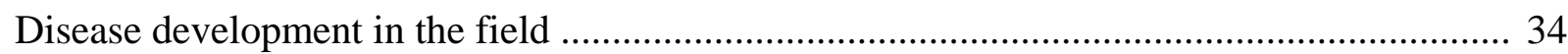

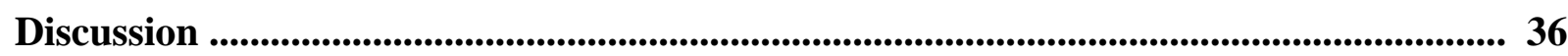

Chapter 3: Differential interactions of Verticillium longisporum and $V$. dahliae with $B$. napus detected with molecular and histological techniques

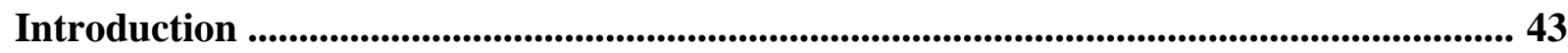

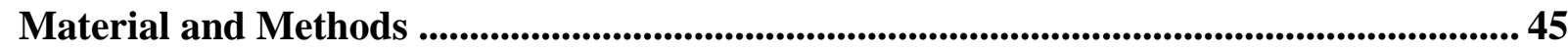




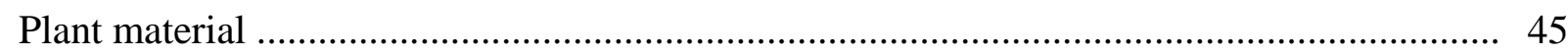

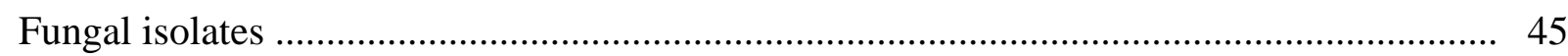

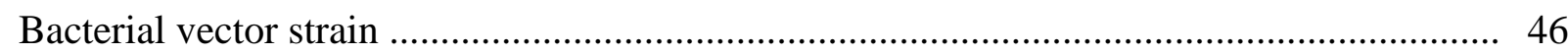

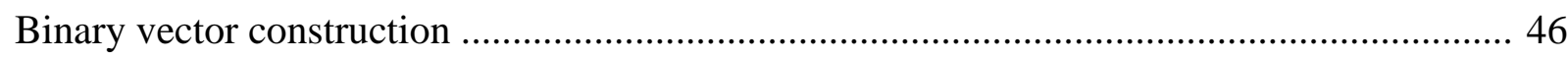

Agrobacterium-mediated transformation of $V$. longisporum and $V$. dahliae ......................... 47

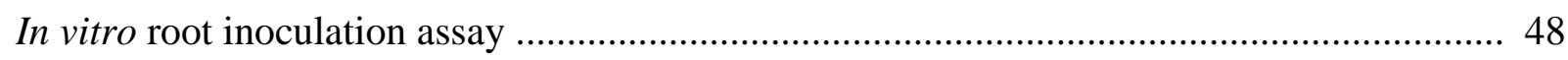

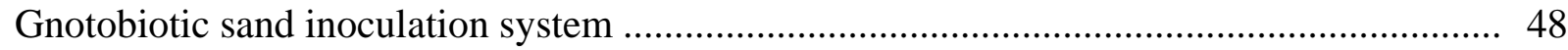

Assessment of disease development ................................................................................ 49

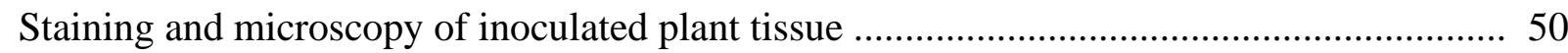

DNA extraction and real-time PCR analyses ........................................................... 51

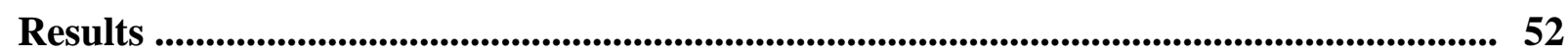

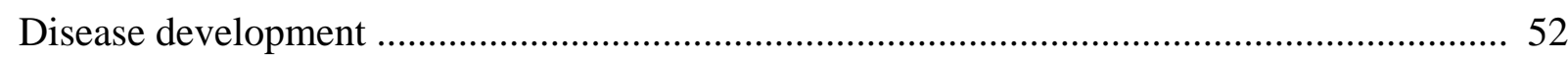

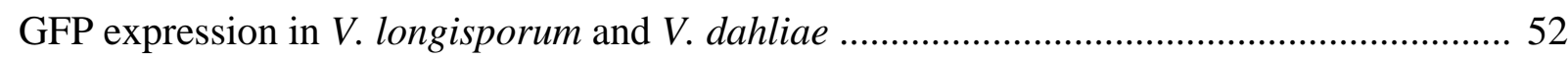

Suitability of GFP-tagged strains vs. classical staining methods ........................................... 53

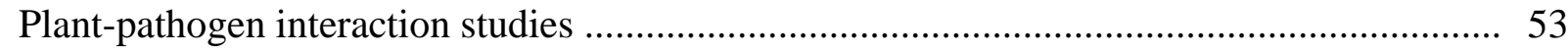

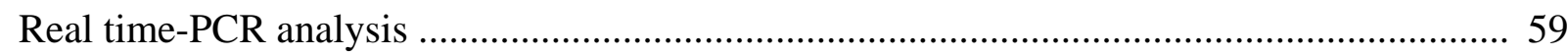

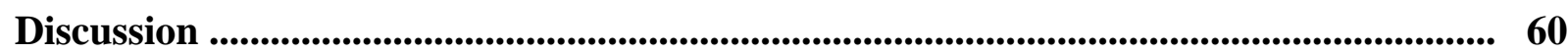

Chapter 4: Mechanisms involved in resistance of Brassica spp. to

V. longisporum .......................................................................................... 63

Introduction ........................................................................................................................................... 63

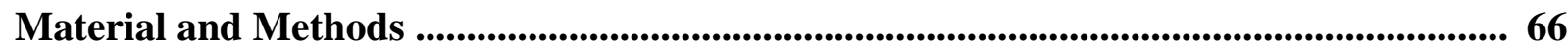

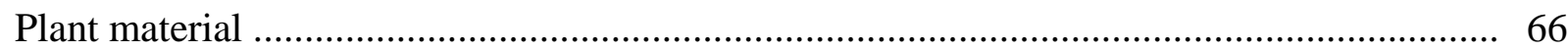

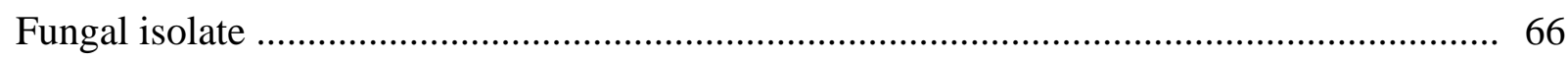

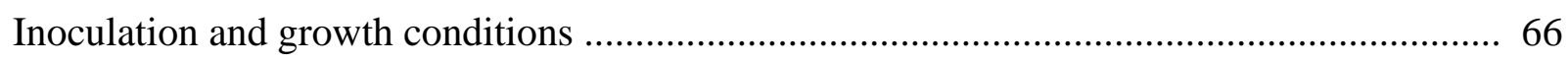

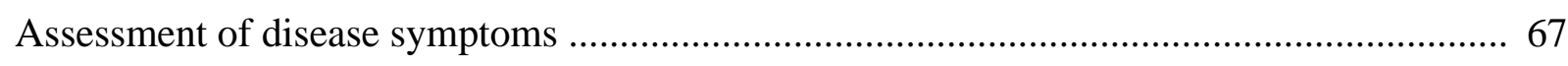

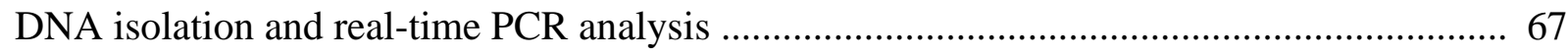

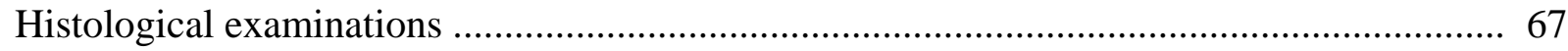

Analysis of soluble phenolic acids, cell wall-bound phenolics and lignin ............................ 68

Quantification of soluble phenolic acids ............................................................ 68

Quantification of cell wall-bound phenolic acids ................................................... 69

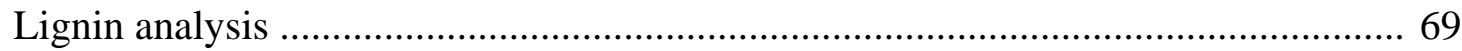

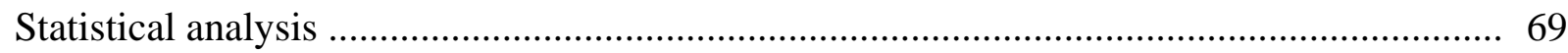

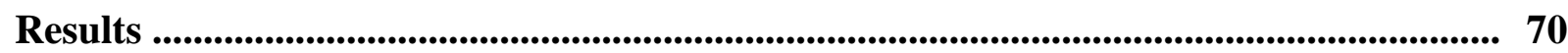




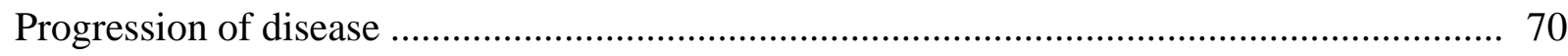

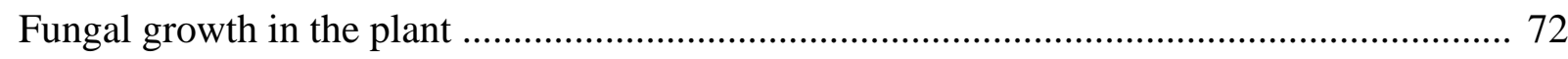

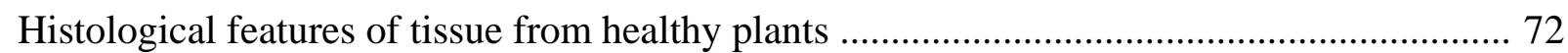

Histological responses in Verticillium-infected B. napus hypocotyls .................................. 73

Quantitative analysis of soluble phenolics, cell wall-bound phenolic acids and lignin .......... 81

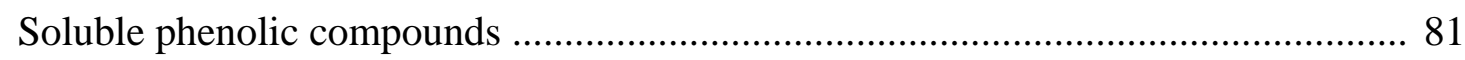

Cell wall-bound phenolic acids ................................................................................ 82

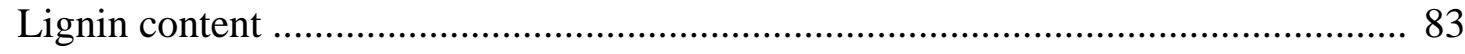

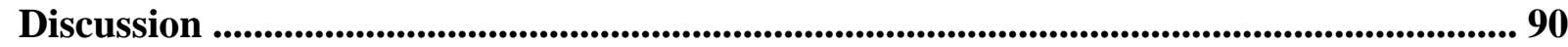

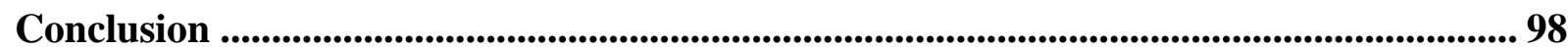

Chapter 5: General Discussion ......................................................................... 99

Host- and non-host- pathogen interaction studies using confocal laser scanning microscopy

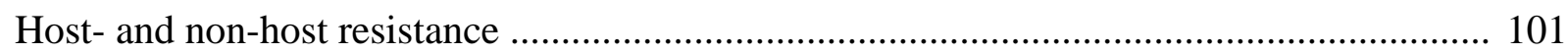

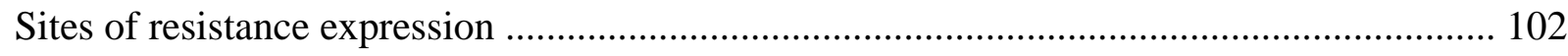

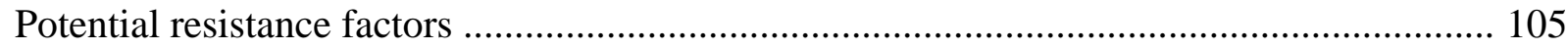

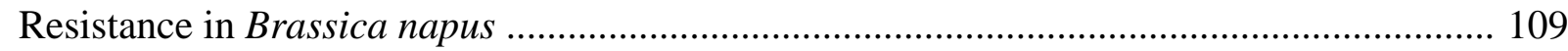

Verticillium longisporum as a model for studying vascular fungal pathogens? ................... 110

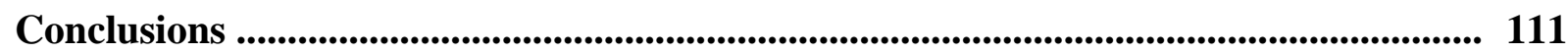

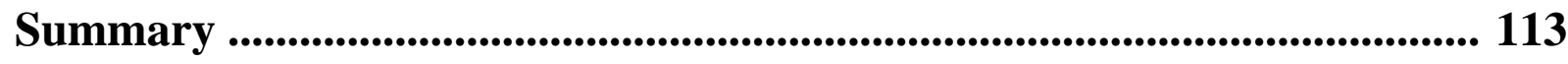

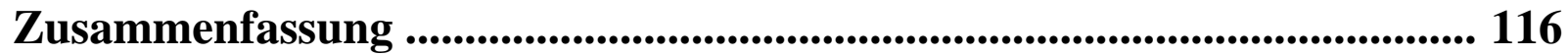

References ...................................................................................................... 119

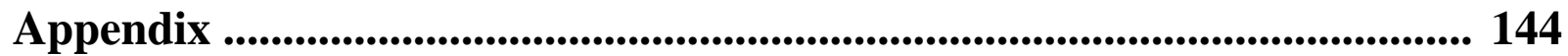

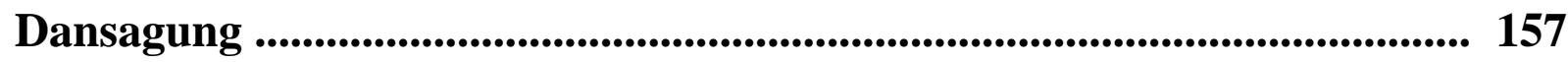

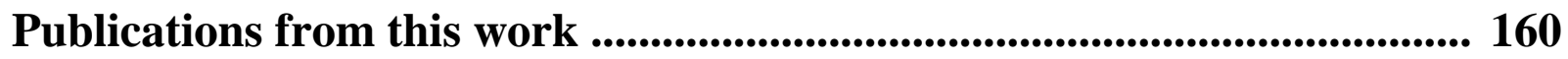

Curriculum Vitae ..................................................................................... 161 


\section{Chapter 1: Introduction}

\section{Aims and objectives of the study}

Oilseed rape (Brassica napus L. oleifera) is at present the most important oilseed crop in Europe. The cultivation area in Germany was more than 1.5 M ha in 2007 (Federal Statistical Office, Germany, 2007; EUROSTAT, 2007). As a result of the increasing area under rapeseed cultivation and the relatively high crop rotation rate disease problems have become a major issue in current breeding efforts.

Verticillium wilt caused by the hemibiotrophic pathogen Verticillium longisporum (ex. V. dahliae var. longisporum Stark; comb. nov. Karapapa) is one of the principal diseases in the cultivation of oilseed rape, besides blackleg and stem canker caused by Phoma lingam and stem rot caused by Sclerotinia sclerotiorum. Soil inoculum in form of microsclerotia can gain densities that lead to a disease incidence of up to $100 \%$ (Zeise \& Steinbach, 2004) in heavily infested fields. The control of these resting structures is particularly difficult because they can survive in the soil for several years (Schnathorst, 1981; Heale \& Karapapa, 1999). Due to the fact that chemical treatments have no effect on the occurrence of $V$. longisporum, the implementation of cultural practices such as crop rotation, time point of sowing, choice of intercrops and the use of resistant cultivars within an integrated disease management approach is the most promising strategy of controlling Verticillium wilt. Unfortunately, until now, breeding for resistance for both winter and spring type oilseed rape has been severely hampered by the absence of sufficient resistance in commercially available breeding material, however, recently, some promising genotypes of cabbage (B. oleracea) and turnip rape (B. rapa) with enhanced resistance were identified (Happstadius et al., 2003; Dixelius et al., 2005). In addition, oilseed rape cultivars with partial resistance also have the potential to reduce crop losses in an environmentally safe, cost-effective manner. Moreover, these cultivars can be combined with other control measurements to achieve optimal levels of disease management (Debode, 2005). Thus, in order to preserve the productivity of the intensive oilseed rape cropping areas in a sustainable, environment-friendly manner, the most promising approach to control Verticillium wilt of oilseed rape is to improve the resistance of the cultivars currently available. This work describes results from a joint project conducted in collaboration with the Institute of Plant Breeding of the Justus-Liebig-University of Giessen and the Association of German Plant Breeders e.V. (GFP) with the ultimate goal of improving the resistance of commercial cultivars to Verticillium. 
The first objective of this work was to seek for sources of resistance in different lines of $B$. oleracea, B. rapa (syn. campestris) and B. napus as well as resynthesized oilseed rape forms to identify genotypes that can be used in future breeding programs. The napus accessions tested included cultivar material as well as breeding lines of the participating breeding companies, the selection of varieties being geared at high-capacity quality material (00quality). B. rapa, B. oleracea as well as resynthesized rapeseed forms served to broaden the genetic variability of the testing material. Resistant $B$. oleracea gene bank material which has been characterized previously by Happstadius et al. (2003) was of particular importance in the project, besides B. rapa and B. oleracea wild species. Thus, a comprehensive greenhouse screening assay, based on former investigations by Zeise (1992), was established, followed by a resistance test of selected $B$. napus accessions under field conditions. The latter was performed at several locations in the North of Germany, namely Fehmarn, Thüle, Futterkamp (season 2005/06) and Rostock (season 2006/07), with a long history of oilseed rape cultivation and a known high natural infestation level. In addition, the selected material from the greenhouse screen was grown on a field site near Göttingen which has been artificially inoculated (Chapter 2).

Based on investigations of napus genotypes and other Brassica species which have been shown to be moderately to highly resistant in preliminary tests, the second objective of this work was to characterize the resistance mechanisms involved. Two different approaches were pursued throughout this project: In the first approach, the interaction of the host-specific species V. longisporum (compatible interaction) with B. napus was compared with the nonhost interaction of $B$. napus with $V$. dahliae. The differential interactions of $V$. longisporum and $V$. dahliae on the root surface and in the root and shoot vascular system of $B$. napus were studied by confocal laser scanning microscopy (CLSM), using GFP tagging and conventional fluorescence dyes. The results of these studies are included in Chapter 3 and focus on the Agrobacterium tumefaciens mediated transformation of both $V$. longisporum and $V$. dahliae resulting in stable expression of GFP, the comparison of the suitability of the GFP reporter gene technique with classical fluorescence staining and the description of yet unknown aspects in the early and advanced stages of infection and colonization of roots and shoots of B. napus by $V$. longisporum and $V$. dahliae.

In the second approach, screening material with different levels of resistance towards $V$. longisporum was used to differentially characterize relevant resistance factors. Based on the results obtained from the studies of the non-host interaction, emphasis was placed on resistance to systemic colonization. Particularly, mechanisms limiting the longitudinal as well 
as the lateral spread of the pathogen in the plant were investigated. This included studies on the formation of mechanical barriers and the kinetics of accumulation of soluble and wallbound phenolics and lignin in a resistant and a susceptible $B$. napus genotype compared to a resistant B. oleracea variety in response to infection with $V$. longisporum (Chapter 4).

The dissertation concludes with a general discussion summarizing the main findings and discussing the potential contributions provided by the work presented.

\section{Literature review}

Introduction

Fungi of the genus Verticillium represent one of the most widespread and devastating groups of plant pathogens worldwide and are frequently encountered in cool and warm temperate regions (Pegg \& Brady, 2002). After comprehensive revision six plant pathogenic species of Verticillium are now generally accepted (Barbara \& Clewes, 2003). The two most important and most studied ones are V. dahliae Klebahn and V. albo-atrum Reinke \& Berthold. While V. dahliae attacks, among others, tomato, pepper, watermelon, mint, muskmelon, lettuce, potato, cotton and strawberry (Gordon et al., 2006), V. albo-atrum causes wilt mainly in alfalfa, hop, tomato and potato (Heale, 2000). Four other species, V. tricorpus Isaac, V. nigrescens Pethybridgs, V. nubilum Pethybridge and V. theobromae Mason \& Hughes are similar but weaker plant pathogens or soil saprophytes. Recently, the proposal has been made for a new Verticillium species, named V. longisporum, including all isolates that are long-spored and host-adapted to Brassica species (Karapapa et al., 1997, 2000).

\section{Taxonomy of Verticillium longisporum}

The occurrence of Verticillium spp. on cruciferous host plants has rarely been described in the past. Only two reports dealt with the isolation of cruciferous Verticillium isolates, from Brussels sprout in the 1950s (Isaac, 1957) and from horseradish in the early 1960s (Stark, 1961). Because of the growing area under rapeseed cultivation and a relatively intense crop rotation the incidence of Verticillium on cruciferous oil crops (particularly oilseed rape and turnip rape) has been reported more frequently since then, especially in Europe (Krüger, 1989; Zielenski \& Sadowski, 1995). In Sweden, for example, Verticillium wilt of oilseed rape, known since the 1960s (Svenson, \& Lerenius, 1987), has become a significant problem since the 1970s (Dixelius et al., 2005). In the main cropping areas for winter oilseed rape in Germany, the occurrence of Verticillium spp. increased rapidly from the mid 1980s (Daebeler 
et al., 1988; Zeise \& Seidel, 1990; Günzelmann \& Paul, 1990). Reports on Verticillium isolates on cruciferous hosts causing serious yield losses have emerged from all over the world (Karapapa et al., 1997; Portenko, 2000; Koike et al, 1994; Eastburn \& Chang, 1994; Kemmochi et al., 2000) since the 1990s.

Since scientists have worked with this fungus, there has been a controversy concerning the recognition of $V$. longisporum as a separate host-specific species. In 1957, Isaac reported on an exceptional isolate of $V$. dahliae from Brussels sprout. From inoculation assays with different Verticillium species on different host plants, he concluded that the fungus isolated from Brussels sprout was a distinct physiological strain with a restricted host range. Stark (1961) for the first time made a differentiation of long-spored Verticillium isolates collected from horseradish, classified as V. dahliae var. longisporum. In 1997, Karapapa et al. proposed a new species, $V$. longisporum, because they were able to clearly distinguish cruciferous Verticillium isolates from isolates of $V$. dahliae and $V$. albo-atrum. Besides the fact that isolates from crucifers produced longer conidia, as had already been noted by Stark (1961), they found additional differences with regard to morphological, enzymatic, molecular and virulence characteristics. Furthermore, due to an about 1.75 fold nuclear DNA content compared to short-spored isolates, they suggested that $V$. longisporum might have emerged from parasexual hybridization between $V$. dahliae and $V$. albo-atrum, therefore explaining its 'near-diploid' state.

The determination of $V$. longisporum as a separate species was questioned by Collins et al. (2003). They argued that the investigations of Karapapa et al. (1997) did not adhere to all long-spored isolates, including the original strain of $V$. dahliae var. longisporum described by Stark (1961). Furthermore, the new taxon V. longisporum did not manage to taxonomically place those crucifer isolates that were short-spored. Based on their results they proposed that the establishment of a new species of long-spored isolates from crucifers was premature and that, by analogy with the terminology used to describe interspecific hybrids in plants, a more appropriate term to describe the retention of two nearly complete haploid genomes in a fused nucleus would be needed such as allodiploid or amphihaploid.

The debate continued with Fahleson et al. (2004) who, based on sequence data, proposed that $V$. longisporum should be regarded as a distinct species closely related to V. albo-atrum. However, with respect to the results of other authors, they admitted that the name $V$. longisporum might be somewhat misleading since within the new species isolates with relatively short conidia could be grouped with long-spored isolates, at least on the molecular level (Steventon et al., 2002a), and different spore lengths have been observed for the same 
isolate (Subbarao et al., 1995; Collins et al., 2003). In their opinion, V. longisporum cannot strictly be related to Brassica host plants since the latter, especially very weak plants, can host several Verticillium species, and V. longisporum can also infect plant species outside the Brassicaceae family (Johannson et al., 2003, 2005). This latter statement contradicts the results of several investigations of Zeise \& von Tiedemann (2001, 2002a, 2002b) who showed that $V$. longisporum is a strictly host adapted pathogen specific for Brassica species. Additional confusion has been caused by some misidentification of $V$. dahliae and $V$. longisporum. In several publications, $V$. dahliae has been considered to be the causal agent of Verticillium wilt in Brassica crops (Xiao \& Subbarao, 2000; Söchting \& Verreet, 2004) or on horseradish (Babadoost et al., 2004), without considering that long-spored isolates may have been involved.

On the basis of microsatellite and other marker analyses, Barbara and colleagues (Barbara \& Clewes, 2003; Barbara et al., 2005; Clewes and Barbara, 2005) addressed the parental origin of the interspecific hybrid Verticillium isolates. They suggested that long-spored crucifer isolates have emerged through parasexual hybridisation between a species that is probably generally similar to other haploid $V$. dahliae isolates and one that is ' $V$. albo-atrum-like' but clearly distinct and of unknown morphology. In summary, the complete nomenclature of the genus Verticillium is still a matter of debate and the understanding of the phylogeny of the strains which infect crucifers is still incomplete. Thus, further research is needed to be able to draw general conclusions.

\section{Life cycle of Verticillium}

$V$. longisporum, like $V$. dahliae and V. albo-atrum, is a soilborne vascular fungal pathogen infecting plants through their roots. It causes a monocyclic disease in the sense that only one cycle of disease with inoculum production occurs throughout a growing season. The life cycle of $V$. longisporum largely equals that of other Verticillium species and can be divided into a dormant, a parasitic and a saprophytic phase. Throughout the dormant phase, which starts when environmental conditions are not suitable for growth, the fungus forms resting structures, so-called microsclerotia, which are melanized aggregates of enlarged hyphal cells which constitute a viable inoculum source in the soil for several years (Schnathorst, 1981; Heale \& Karapapa, 1999). Microsclerotia must withstand microbial degradation, lysis, parasitism and predation, and therefore present an important trophic level in the soil ecosystem (Okubara \& Paulitz, 2005). The germination of microsclerotia is inhibited via microbiostasis or mycostasis (Fradin \& Thomma, 2006), which is compromised by root 
exudates released into the rhizosphere of host or non-host plants (Schreiber \& Green, 1963; Huisman, 1982; Olsson \& Nordbring-Hertz, 1985; Mol et al., 1995; Gödecke, 2007, unpublished results). Supposedly following a nutrient gradient, hyphae which grow out of germinating microsclerotia are able to traverse a limited distance in the soil in order to reach the roots of potential host plants. Thus, it has been calculated that the sphere of influence of any root on microsclerotia of $V$. dahliae is about $300 \mu \mathrm{M}$ wide (Huisman, 1982).

While entering the parasitic phase, Verticillium fungi are able to directly penetrate the epidermal cells of the root. Nevertheless, it is generally assumed that natural openings like sites of lateral root formation as well as wounds caused by nematodes or root feeding insects ease the ingress of the pathogen. In order to reach the xylem vessels the fungus crosses the root cortex and the endodermis which acts as a physical barrier due to suberin depositions (Talboys, 1958; Bishop \& Cooper, 1983a). Several authors have proposed that crossing of the endodermis is accomplished when it is damaged or through penetration and longitudinal growth from regions near the root tip, which are devoid of a mature endodermis (Pegg, 1974; Schnathorst, 1981; Huisman, 1982; Bowers et al., 1996). Afterwards, the fungus colonizes the vascular system and does not leave this environment until senescence and maturity stages of the host plant. Thus, most of the time of its life cycle Verticllium is confined to the vascular system. The fungal spread in this environment is mainly achieved via spore release because colonization by vegetative growth would be too slow due to the poor mycelial growth rate (Presley et al., 1966). As observed for other Verticillium species (Buckley et al., 1969), V. longisporum does not form verticillate conidiophores (own observations) in the plant tissue, thus conidia are probably produced by simple conidiophores or by simple terminal or lateral conidiation (budding). Conidia are carried upwards with the transpiration stream and trapped in pit cavities or at vessel end-walls (i.e. trapping-sites). For the colonization to continue, conidia germinate and germ tubes penetrate through full-bordered pits into adjacent vessel elements (Garas et al., 1986), thus beginning another infection cycle. This mode of host colonization may account for both the observed discontinuous occurrence of mycelium in the plant vascular system (Heinz et al., 1998; Beckman, 1987) and the rapidity with which systemic colonisation occurs (Zinkernagel, 1982; Gold \& Robb, 1995; Heinz et al., 1998; Chen et al., 2004).

In the later stages of the disease cycle, as the host begins to senesce, the pathogen enters a limited saprophytic growth phase in which microsclerotia are formed in the dying stem parenchyma, (Schnathorst, 1981; Neumann \& Dobinson, 2003). 


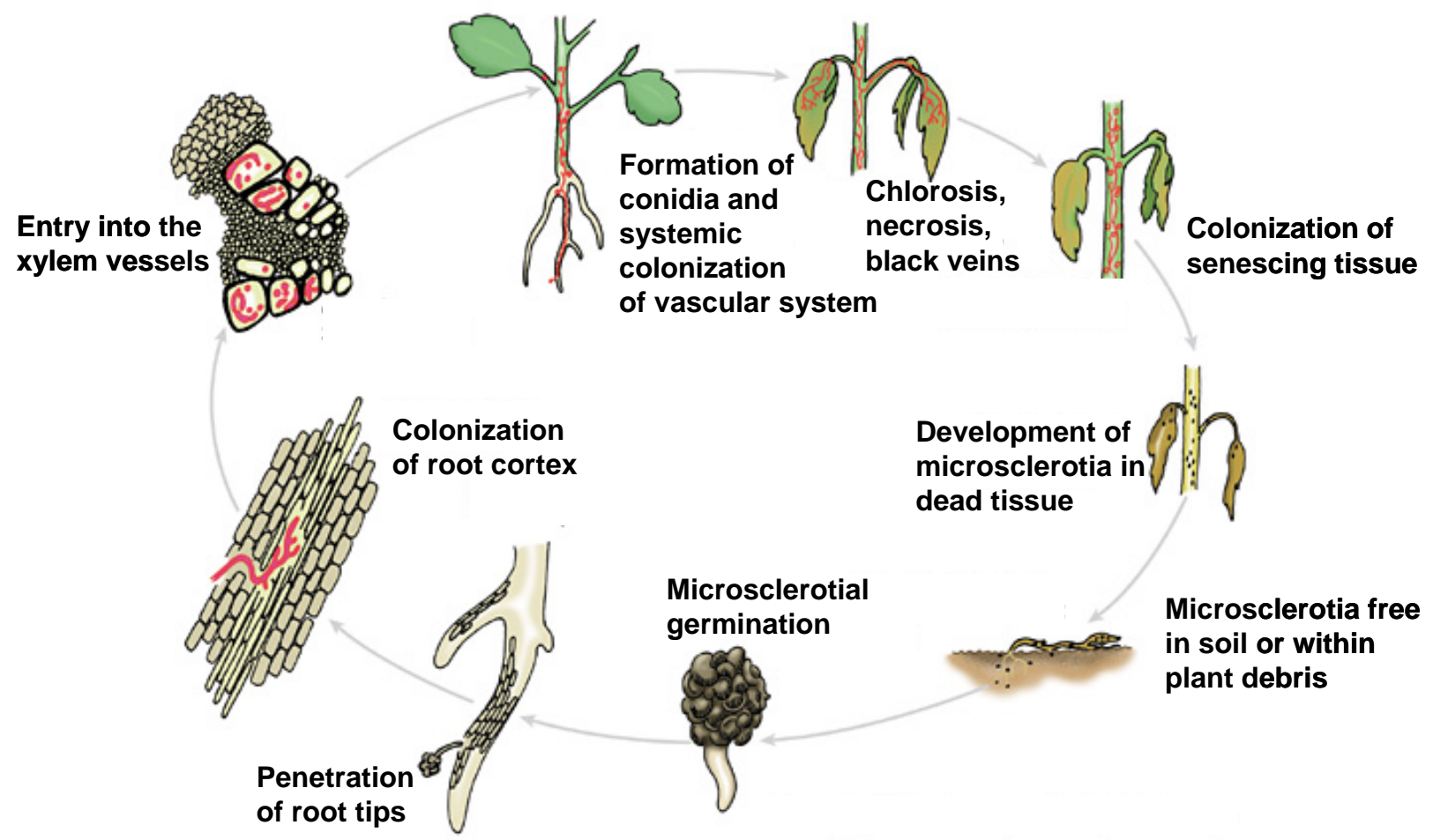

(modified according to Brewster, V., Ewing, J.)

Fig. 1.1. Life cycle of V. dahliae / V. longisporum. From Rowe \& Powelson (2002).

In contrast to diseases induced by other Verticillium species, V. longisporum does not induce wilt symptoms in oilseed rape. Due to the fact that colonization of the plant by $V$. longisporum in the field is characterized by an extended latent phase, early symptoms like streaky yellow to brownish discolorations of the stem and half-sided yellowing of the leaves as well as chlorotic patches between veins which themselves turn blackish, cannot be observed before the beginning of plant maturity after which the pathogen bursts out of the xylem vessels to produce ample masses of microsclerotia underneath the stem epidermis, in the stem pith and in the roots. Further symptoms of the disease are premature bloom and maturity as well as stunted growth, the latter occurring particularly under standardised conditions in the greenhouse or climate chamber. With these considerations, a more appropriate term to describe the disease caused by $V$. longisporum on crucifers would rather be Verticillium premature senescence (VPS) or Verticillium premature ripening (VPR) than Verticillium wilt. 


\section{Resistance to Verticillium}

According to Pegg \& Brady (2002) resistance to Verticillium can be defined as 'the total or partial absence of symptoms in comparison with other host species or cultivars similarly exposed to virulent pathotypes and showing severe damage or death'. Because of the systemic nature of Verticillium infections, the cellular bases of resistance and susceptibility to wilt disease have been difficult to measure (Gold \& Robb, 1995). However, resistance to Verticillium has mostly been studied in cotton (Hill et al., 1999), hop (Talboys, 1957, 1958a) and the solanaceous crops tomato (Bishop \& Cooper, 1984; Gold \& Robb, 1995) and potato (Concibido et al., 1994; Lynch et al., 1997; Jansky, 2000). In cruciferous host plants, resistance to Verticillium has been described in cauliflower (Koike \& Subbarao, 1994; Debode, 2005), Japanese cabbage (Kemmochi et al., 2000), horseradish (Atibalentja \& Eatburn, 1998), cabbage and turnip rape (Happstadius et al., 2003; Dixelius et al., 2005), and in oilseed rape (Steventon et al., 2002b).

Resistance mechanisms against Verticillium may become effective either during initial (prepenetration) or later (post-penetration) stages of infection. According to Talboys (1957), this later stage can be divided into two further phases with the somewhat misleading terms determinative phase (establishment of the fungus in the root) and expressive phase (development of symptoms in aerial parts of the host), probably more appropriately termed as prevascular and vascular phase (Talboys, 1964).

With respect to defence responses taking place during the prevascular phase, the formation of cell wall appositions and papillae (also called lignitubers) in root cells preventing further colonization by the fungus was reported from several authors (Van der Meer, 1925; Talboys 1958; Presley et al., 1966; Griffiths, 1971; Bishop \& Cooper 1983a). Much uncertainty exists with regard to the role and chemical composition of these lignitubers and their role in resistance is unequivocal. Thus, according to Bishop and Cooper (1983a), the frequency of apposition formation appeared similar in both resistant and susceptible varieties, indicating that a significant role in resistance is unlikely. Furthermore, the role of penetration papillae in plant defence has been questioned by Wood (1967) due to the fact that they were also generated as a response to mechanical stress. In general, no correlation has been confirmed between genetic resistance and lignituber formation.

The suberinized endodermis is generally thought to act as a natural barrier against Verticillium infection in roots (Talboys, 1958), although penetration into stelar parenchyma through endodermal cells was observed. Finally, the production of phytoalexins which may act as 
antimicrobial agents is reported to be induced in the root tissue upon infection (Bell, 1969; Daayf et al., 1997).

Another set of factors which has been found to be important regarding plant defence against Verticillium spp., comprises those governing resistance and susceptibility to systemic colonization during the vascular phase. According to Pegg \& Brady (2002) resistant plants hold a pronounced capability of quickly inducing defence responses in the vessels and thus containing the fungus in the root and lower stem tissue, whereas in susceptible plants the fungus succeeds to escape from plant defence. Often, the success of defence responses seems to depend on the ability of the plant to rapidly restrict the systemic spread of the pathogen with a combination of constitutive and induced defence mechanisms (Bell, 1994). Unlike most biotrophic and some necrotrophic interactions, there is no total resistance to infection. Induced defence mechanisms include, among others, vascular occlusion due to the production of vascular gums, gels and tyloses (Beckman \& Talboys, 1981; Beckman, 1987; Benhamou, 1995) which serve to impede the longitudinal spread of the pathogen due to the trapping of released conidia. In addition, as has been described for tomato plants resistant towards $V$. albo-atrum and V. dahliae, the deposition of suberin and other coating materials on vascular cell walls was reported to form a barrier preventing horizontal dispersal of the fungus (Beckman, 1987; Robb et al., 1984, 1989; Street et al., 1986).

Furthermore, the induction of antimicrobial compounds, such as the pathogenesis-related proteins (PR proteins) chitinase and ß-1,3-glucanase (Dubery \& Slater, 1997), and phytoalexins (Cooper et al., 1996; Williams et al., 2002), such as the flavonoid and terpenoid phytoalexins of cotton (Mace et al., 1990; Joost et al., 1995) as well as the accumulation of phenolic compounds (Beckman, 2000) have been suggested to play a pivotal role as resistance mechanisms. The importance of phenolic compounds in plant defence has also been stressed by molecular studies on tomato plants infected with $V$. albo-atrum which revealed that the fungus is able to suppress the transcription of phenylalanine ammonia lyase (PAL) genes in susceptible plants (Lee et al., 1992). PAL is the first key enzyme in the phenylpropanoid pathway and therefore its expression may have an effect on the regulation of phenylpropanoid-based defence compounds such as suberin (Hahlbrock \& Scheel, 1989), lignin (Smit and Dubery, 1997), phytoalexins and some plant hormones (Jones, 1984; MauchMani \& Slusarenko, 1996).

The interplay between physical and antimicrobial defence responses might reveal an efficient way to restrict fungal spread. Thus, it has been suggested by many authors that the fungus is first trapped in so-called trapping-sites, ensued by the elimination of fungal hyphae due to the 
release of antimicrobial compounds (Sinha \& Wood, 1967; Dixon \& Pegg, 1969; Benhamou, 1995; Gold \& Robb, 1995; Chen et al., 2004).

The only inorganic and therefore most striking phytoalexin contributing to Verticillium resistance is elemental sulphur, which has long been known as a fungicide used in orchards (Smith et al., 1995a; Warkentin et al., 1996) and vineyards (Jolivet, 1993). In both resistant cocoa and tomato plants this element has been found to accumulate in concentrations in xylem vessel walls and xylem occluding gels sufficiently high to control fungal growth (Cooper et al., 1996; Williams et al., 2002, Williams \& Cooper, 2003, 2004; Cooper \& Williams, 2004).

Many plants have been found encoding a distinct set of cell wall-associated glycoproteins able to inhibit endopolygalacturonase enzymes which are produced by fungal pathogens, the socalled polygalacturonase-inhibiting proteins, PGIPs (De Lorenzo et al., 2001). It has been proposed that PGIPs and resistance gene products may function as integrated components of a cell surface apparatus that forms part of the plant's immune system (De Lorenzo \& Cervone, 1997). As a matter of fact, PGIPs have been isolated from cotton (James \& Dubery, 2001) and apple (Gazendam et al., 2004) and displayed an inhibitory effect on polygalacturonases of $V$. dahliae in vitro; however, their effect in vivo has yet to be determined. Recently, two PGIPencoding genes from B. napus have been characterized (Li et al., 2003) but knowledge as to their role in defence towards $V$. longisporum is still lacking.

For the vascular pathogen Ophiostoma novo-ulmi on elms it has been demonstrated that the anatomy of the vascular system has a pivotal impact on the development of the disease but this has not yet been reported for Verticillium. Accordingly, wider and successional vessels favour the spread of the fungus, its propagules and metabolites (Elgersma, 1970; Mc Nabb et al., 1970; Solla \& Gil, 2002). Likewise, the length and the extent of branching of vessels might have an influence on fungal dispersion (Van Alfen, 1989). Thus, the absence of vascular pathogens in gymnosperms is related to the short length of tracheid elements (Pegg $\&$ Brady, 2002). Furthermore, the differentiation of new xylem vessels from interfascicular cambium following infection with $V$. albo-atrum was reported for wilt-tolerant hop cultivars (Talboys, 1958b). In this respect, the net increase in fungus-free xylem was assumed to act as a compensatory resistance mechanism.

Most of the mechanisms referred to may contribute to a general expression of resistance rather than to function alone. Above all, they represent non-specific responses, some of which can be found in susceptible as well as resistant hosts (Beckman \& Talboys, 1981; Beckman, 1987). 
The genetic basis and the molecular mechanisms underlying plant defence responses to Verticillium are poorly understood. Many crop species, such as alfalfa and mint contain genes coding for tolerance, but not complete resistance, to Verticillium spp. (Hastie \& Heale, 1981). The resistance of tetraploid cultivated potato (Solanum tuberosum L.) to V. albo-atrum and V. dahliae appears to be polygenic in nature (Simko et al., 2004), and is based predominantly on the additive effect of minor genes (Hunter et al., 1968) whereas in diploid potato hybrids resistance seems to be inherited in a single dominant manner (Lynch et al., 1997; Jansky et al., 2004). Polygenic resistance has also been reported for cotton (Bolek et al., 2005) and strawberry (Lynch, 1997).

The only known Verticillium resistance gene is the tomato Ve gene, which encodes a leucinrich repeat (LRR) protein belonging to the class of so-called receptor-like proteins (Kruijt et al., 2005) and has been fully characterized by Kawchuk et al. (2001). It is inherited in a monogenic, dominant fashion (Gold \& Robb, 1995) and confers resistance to race 1 particularly, of both V. albo-atrum (Kawchuk et al., 2001) and V. dahliae (Schaible, 1951; Diwan et al., 1999). However, it is not known where and how Ve-mediated resistance is established in the plant. According to Fradin \& Thomma (2006) the Ve resistance may possibly be operative in the parenchyma cells surrounding xylem vessels, a phenomenon that has already been implicated with the tomato $I-2$ gene which provides resistance to the vascular fungal pathogen Fusarium oxysporum (Mes et al., 2000).

In recent years, great efforts have been made to identify genes involved in resistance to Verticillium in cruciferous plants. In Arabidopsis thaliana, Veronese et al. (2003) have identified a single dominant locus, Verticillium dahliae-tolerance (VET1) which governs the severity of symptom development, particularly with regard to the ageing process. However, this allele neither led to the reduction of pathogen growth nor did it have any influence on the ability of the pathogen to induce the expression of defence genes which are controlled by different response pathways. According to Kemmochi et al. (2000), resistance of Japanese cabbage is polygenic and inherited in a dominant manner. Quantitative inheritance of Verticillium resistance is also assumed for cauliflower, however, resistance genes have not yet been identified (Happstadius et al., 2003; Debode, 2005). 


\section{Chapter 2: Identification of Brassica accessions with enhanced resistance to Verticllium longisporum under controlled and field conditions*}

*) Parts of this chapter have been submitted for publication in: C. Eynck, B. Koopmann, A. v. Tiedemann (2008) Identification of Brassica accessions with enhanced resistance to Verticillium longisporum under controlled and field conditions

Plant Pathology and Plant Protection Division, Department of Crop Sciences, Faculty of Agriculture, Georg-August University Göttingen, Grisebachstraße 6, D-37077 Göttingen, Germany

\section{Introduction}

Oilseed rape (Brassica napus L. oleifera) is a relatively young species, compared to other crops; however, due to intensive breeding for seed and oil quality traits, oilseed rape nowadays represents one of the most important sources of vegetable oil worldwide (Kimber \& Mc Gregor, 1995), not only for human nutrition but also for livestock feeding and the oleochemical industry (Murphy, 1996). The area under oilseed rape cultivation continues to rise dramatically, because of the high demand for rapeseed oil. This is accompanied by crop rotation cycles which are getting constantly shorter.

As a consequence, Verticillium wilt has become an increasing threat to oilseed rape production particularly in Northern Europe (Krüger, 1989; Zielienski \& Sadowski, 1995). While having become a prevalent disease in Germany (Daebeler et al., 1988; Günzelmann \& Paul, 1990; Zeise \& Seidel, 1990) and Sweden (Svenson \& Lerenius, 1987; Dixelius et al., 2005), Verticillium wilt has also been reported from Poland, France, Russia and the Ukraine (Heale \& Karapapa, 1999).

Verticillium wilt on Brassica oil crops is caused by the soilborne fungus and vascular pathogen Verticillium longisporum (ex. V. dahliae var. longisporum Stark; comb. nov. Karapapa), which quite recently has been considered as being a distinct taxonomic unit separate from other Verticillium species such as $V$. dahliae (Karapapa et al., 1997). The distinction of $V$. longisporum is based on the larger conidia, nuclei containing nearly twice as much DNA due to amphihaploidy, enzymatic characteristics and a significantly higher pathogenicity on cruciferous host plants (Steventon et al., 2002a, b; Zeise \& von Tiedemann, 2002a, b). The proposition of establishing $V$. longisporum as a distinct species taxon was supported by comprehensive molecular analyses done by Steventon et al. (2002a) and Pantou et al. (2006). However, there is still controversy regarding the recognition of $V$. longisporum 
as a separate, host-adapted species (Fahleson et al., 2004). Thus, according to Collins et al. (2003), also short-spored Verticillium isolates can infect cruciferous plants, while $V$. longisporum has been reported to infect plant species which do not belong to the Brassicaceae (Fahleson et al., 2004).

The infection and colonization behaviour of $V$. longisporum exhibits many similarities to that of other Verticillium species, such as V. dahliae and V. albo-atrum. After germination of microsclerotia, which is induced by root exudates of host as well as non-host plants (Olsson \& Nordbring-Hertz, 1985; Mol et al., 1995; Gödecke, 2007, unpublished results), the fungus infects the roots, entering mainly near the root tip. Hyphae cross the root cortex inter- and intracellularly and enter the vessel elements. From here on, the fungus spreads systemically in the vascular system by means of mycelium and conidia released into the transpiration stream (Zhou et al., 2006; Eynck et al., 2007). When senescence of the plant begins, the fungus leaves its vascular environment and produces ample masses of microsclerotia in the dying plant tissue. Soil management practices, in which infested plant material is buried in the soil, provide a pivotal role to microsclerotia as primary inoculum source for the following seasons (Schnathorst, 1981; Neumann \& Dobinson, 2003).

Due to the fact that microsclerotia are able to survive in the soil for several years (Heale \& Karapapa, 1999) and as the application of fungicides is not effective to control $V$. longisporum, resistant cultivars are required (Mert \& Karakaya, 2004). Resistance to Verticillium spp. is known from several other crops, like tomato (Diwan et al., 1999), potato (Simko et al., 2004; Jansky et al., 2004), cotton (Bolek et al., 2005) and strawberry (Lynch, 1997). However, the molecular mechanisms and the genetic basis underlying such plant defence responses to Verticillium are poorly understood. The only known Verticillium resistance gene is the tomato $V e$ gene, which encodes a leucin-rich repeat (LRR) protein belonging to the class of so-called receptor-like proteins (Kruijt et al., 2005). In recent years, breeders have made an extensive effort to identify sources of resistance to $V$. longisporum in the primary oilseed rape gene pool, however without much success (Happstadius et al., 2003). The problem is aggravated by the fact that intensive selection for quality traits has resulted in a narrowed genetic basis of current breeding lines (Becker et al., 1995; Hasan et al., 2005). Nevertheless, also oilseed rape cultivars with partial resistance have the potential to reduce crop losses in an environmentally safe, cost-effective manner. Moreover, cultivars with partial resistance can be combined with other control measurements to achieve optimal disease management (Debode et al., 2005). Furthermore, because B. napus is derived from interspecific hybridisation between turnip rape (B. rapa L. syn. campestris) and cabbage (B. 
oleracea L.) the secondary gene pools of these two progenitor species may also serve as potential gene donors in broadening the resistance base. Recently, some promising genotypes of B. oleracea and B. rapa with enhanced resistance were identified (Happstadius et al., 2003; Dixelius et al., 2005; Rygulla et al., 2007).

Disease screens conducted in infested field sites represent the most accurate and reliable approach to evaluate the resistance response of a certain plant genotype to a pathogen, particularly when it is soilborne. However, field screening has limitations concerning the number of variants possibly tested and due to a heterogeneous disease pressure deriving from variations of environmental factors and the soil inoculum distribution. Due to these constraints most tests to identify resistance in Brassica spp. to Verticillium have been performed under greenhouse conditions (Zeise, 1992; Subbarao et al., 1995; Zeise \& Buchmüller, 1997; Happstadius et al., 2003; Debode et al., 2005). However, it remains unclear whether accessions performing well in controlled conditions would also show an enhanced level of resistance in the field.

Accordingly, the objectives of this work were (i) to screen different Brassica genotypes for resistance towards $V$. longisporum under greenhouse conditions in order to identify resistant or partially resistant varieties for use in future breeding programs, (ii) to test to which extent the results obtained under standardised conditions are valid in the field and (iii) to investigate the time course of $V$. longisporum development under field conditions in oilseed rape plants depending on their particular susceptibility.

\section{Materials and Methods}

Plant material in the greenhouse

In total, 1230 accessions of B. napus, supplied by eight plant breeding companies (Deutsche Saatveredelung Lippstadt Bremen GmbH (DSV), Klein Wanzlebener Saat AG (KWS), Limagrain Nickerson GmbH (NICK), Svalöf Weibull Seed Hadmersleben GmbH (SEM), Syngenta Seeds GmbH (SYN), Raps GbR Saatzucht Lundsgaard (SRG), Norddeutsche Pflanzenzucht Hans-Georg Lembke KG (NPZ), W. von Borries-Eckendorf GmbH \& Co., WVB), were tested in this study. In addition, a total of 80 B. rapa gene bank accessions, including seven cultivar groups, and 33 B. oleracea gene bank accessions, representing six of the fourteen cultivar groups of B. oleracea, were screened for susceptibility to $V$. longisporum in order to identify resistant genotypes useful for breeders to generate resynthesized oilseed rape lines potentially comprising the combination of different resistance loci. These accessions were obtained from eight different gene banks and three commercial seed suppliers 
listed in Table 2.4. Furthermore, thirteen accessions with non-defined species identity and origin were involved in the resistance test. These were supplied by the breeding company DSV. The interspecific hybridisation of B. rapa and B. oleracea resistance sources identified throughout this screening and the generation of resynthesized oilseed rape lines with improved resistance to $V$. longisporum is described in detail by Rygulla et al. (2007a, b).

Inoculation and screening procedure in the greenhouse

Resistance tests were performed with the V. longisporum isolates VL 40 and VL 43, which originate from diseased B. napus plants sampled in the North of Germany. These isolates were chosen on the basis of results of preliminary virulence tests of several Verticillium strains from B. napus described by Zeise \& von Tiedemann (2002a). Long-term storage of fungi was performed as conidial suspensions in a concentration of $1-3 \times 10^{6}$ conidia $\mathrm{mL}^{-1}$ in Czapek-Dox medium (Czapek, 1902; Dox, 1910) supplemented with 25\% glycerol. Inoculum was produced by adding $500 \mu \mathrm{L}$ of spore stock solutions to $250 \mathrm{~mL}$ potato dextrose broth. The cultures were subsequently incubated for 7 days at $23^{\circ} \mathrm{C}$ on a rotary shaker (100 RPM). The resulting suspension was filtered through sterile gauze. Spore concentration was determined with a haemocytometer and diluted to give $1 \times 10^{6}$ spores $\mathrm{mL}^{-1}$.

The winter oilseed rape varieties 'Express' (moderately tolerant) and 'Falcon' (highly susceptible) were used as reference controls in all experiments. Seeds of the control and test plants were surface-sterilized by immersion in $70 \%$ ethanol for $15 \mathrm{~min}$. Subsequently, seeds were washed in sterilized tap water before being sown in double-autoclaved silica sand. Seedlings appeared after 3-7 days, and another 7 days later the roots were carefully washed out of the sand. Inoculation was performed by cutting $2 \mathrm{~cm}$ off the root apex and submerging the capped roots for $30 \mathrm{~min}$ in a mixed conidial suspension of both $V$. longisporum isolates VL 40 and VL 43. Roots of control plants were also cut and submerged in tap water for the same length of time. A total of 20 inoculated seedlings and 20 control plants of each accession were transferred into a mixture of sand, peat and compost $(1: 1: 2)$ in pots containing two plants each. Plants were grown at $23^{\circ} \mathrm{C}$ in a greenhouse with a lighting regime of $14 / 10 \mathrm{~h}$ (light/dark). Plants were not fertilized during these experiments. Weekly scoring of each plant for disease symptoms took place over a four-week period using a slightly modified assessment key (Table 2.1) from Zeise (1992). 
Tab. 2.1. Assessment key for scoring disease severity induced by Verticillium longisporum on Brassica plants in the greenhouse (modified according to Zeise, 1992).

\begin{tabular}{|c|c|}
\hline Score & Symptom development \\
\hline 1 & no symptoms \\
\hline 2 & slight symptoms on the oldest leaf (yellowing, black veins) \\
\hline 3 & slight symptoms on the next younger leaves \\
\hline 4 & about $50 \%$ of the leaves show symptoms \\
\hline 5 & more than $50 \%$ of the leaves show symptoms \\
\hline 6 & up to $50 \%$ of the leaves are dead \\
\hline 7 & more than $50 \%$ of the leaves are dead \\
\hline 8 & only apical meristem is still alive \\
\hline 9 & the plant is dead \\
\hline
\end{tabular}

For each accession the area under the disease progress curve (AUDPC) was calculated from the disease severity values taken four times at weekly intervals, modified according to the following formula from Campbell and Madden (1990):

$$
\mathrm{AUDPC}=\sum_{i=1}^{n}\left(y_{i}+y_{i+1} / 2\right) *\left(t_{i+1}-t_{i}\right)
$$

where $y_{i}$ is the disease severity value for observation number $i, t_{i}$ is the number of days after inoculation at the time point of observation number $i$, and $n$ is the number of observations. In order to consider disparities with regard to the variation in the natural senescence of the different accessions, so as not to overestimate the disease levels of the inoculated variant, an AUDPC value was also calculated for the control variants. This value was subtracted from the AUDPC value of the inoculated variant to obtain the 'net AUDPC value' (AUDPC $\left.{ }_{\text {net }}\right)$ :

$$
A U D P C_{n e t}=A U D P C\left(\mathrm{X}_{\text {inoc. }}\right)-A U D P C\left(\mathrm{X}_{\text {contr. }}\right)
$$


In order to compensate for fluctuating infection levels between trials a normalization of the AUDPC values for each accession was performed based on the reaction of the internal reference controls. The normalized value $\left(\mathrm{AUDPC}_{\text {norm. }}\right)$ was calculated as follows:

$$
A U D P C_{\text {norm. }}=\frac{A U D P C_{\text {net }}(\mathrm{X})}{\left(A U D P C_{\text {net Express }}+A U D P C_{\text {net Falcon }}\right) / 2}
$$

Due to the fact that an infection with $V$. longisporum results in diminished growth, the plant height was measured at $28 \mathrm{dpi}$. The stunting effect was expressed by performing a normalization procedure in analogy to the approach taken for the $\mathrm{AUDPC}_{\text {norm. }}$ values.

Sites and design of field experiments

When new resistance sources are identified under standardized conditions, a further important aspect is to verify these results under natural conditions. Thus, subsets of accessions that had been screened in the greenhouse for their resistance response towards $V$. longisporum were tested in the field. Experiments were conducted from 2004 to 2007.

No appropriate accessions were available for sowings in summer 2004 because the project had just started shortly before. Therefore, promising new winter oilseed rape cultivars such as 'Smart', 'Trabant', 'Oase', 'Mohican' and winter oilseed rape cultivars already established on the German market such as 'Talent' and 'Elektra' were chosen. According to the greenhouse assay, cultivars 'Express' and 'Falcon' served as tolerant and susceptible control varieties, respectively. Prior to sowing, oilseed rape straw, naturally infested with microsclerotia, was deployed and incorporated into the upper soil layers, resulting in $15 \mathrm{~g}$ inoculum material per $\mathrm{m}^{2}$. This material originated from highly infested stubbles that had been collected after the previous harvest, dried and shredded. Accessions were seeded as a randomized complete block design with four replications at a location near Goettingen, Lower Saxony. At this site oilseed rape was not grown for at least ten years, thus natural infestation levels were kept to a minimum. The trial was maintained using standard cultural practices for oilseed rape production in Germany with the control of Phoma lingam and Sclerotinia sclerotiorum by spraying fungicides at the appropriate growth stages. To measure the extent of infestation as well as disease dynamics, samples (5 per plot, fourfold repetition), comprising the root and the lower stem part, were taken from each accession at regular intervals throughout the vegetation period. Subsequently, samples were examined by ELISA in order to determine the 
amount of $V$. longisporum biomass in the particular sample. Additionally, visual scoring of disease severity was carried out on stubbles soon after harvest on 50 stubbles per plot.

In 2005 three to five accessions of each participating breeding company was sown for further resistance testing in the field. In addition to the tolerant and susceptible standards 'Express' and 'Falcon' three further reference cultivars, which had been characterized in the greenhouse for their response to V. longisporum, were used. Those were the cultivars 'Smart' and 'Lion' with high and even very high tolerance to the pathogen, whereas cultivar 'Laser' was highly susceptible to $V$. longisporum. Besides the site near Goettingen, where the field was artificially inoculated by the same procedure as in the previous year, resistance trials were run on three further locations in the North and Northeast of Germany (Futterkamp and Fehmarn in Schleswig-Holstein, Thuele in Lower Saxony). These sites were thought to exhibit a high natural infection pressure, based on observations of disease incidence in previous years. Due to the limited availability of seeds, each accession was sown only in twofold repetition on each site. While root and stem material from all sites was visually scored after harvest (25 stubbles per plot), in Goettingen, as in the season 2004/05, additional samples (5 per plot) were taken consecutively throughout the vegetation period to document the occurrence and systemic spread of the pathogen in plants of particular accessions with ELISA.

In 2006 one further breeding company (Pflanzenzucht Dr. h.c. Carsten, Lübeck, Germany, DM) participated in the resistance tests in the open field. A total of forty accessions were tested, comprising 36 selected by the participating breeders (four accessions per breeder) and four reference varieties. Besides 'Falcon' and 'Express', the latter comprised the cultivars 'Oase' and 'Laser' as highly tolerant and highly susceptible reference varieties, respectively. The experimental sites were the same as in the previous seasons. Due to an extremely low natural infestation level in Futterkamp in the vegetation period 2005/06 this site was replaced by a location near Rostock (Mecklenburg-Western Pomerania). As in the previous year, 25 stubbles per plot were collected after harvest from each experimental site for visual disease scorings. Plant density and cultural practices were identical in all three years. 
Visual scoring of $V$. longisporum disease symptoms in the field

Immediately after harvest, 50 (in 2005) and 25 (in 2006 and 2007) stubbles were collected from each plot at each experimental location. Disease severity and disease incidence due to $V$. longisporum were scored visually based on the amount of microsclerotia under the stem epidermis, in the stem pith and in the roots for each plant, with the help of the rating scale represented in Table 2.2.

Tab. 2.2. Rating scale used to score the disease severity on stubbles collected in the field.

\begin{tabular}{l|l}
\hline Score & Symptom \\
\hline 1 & $\begin{array}{l}\text { healthy, no microsclerotia visible } \\
\text { slight colonization with microsclerotia } \\
2\end{array}$ \\
3 & $\begin{array}{l}\text { strong colonization with microsclerotia } \\
\text { very strong colonization with microsclerotia, epidermis is pealing off } \\
\text { (stem) }\end{array}$ \\
\hline
\end{tabular}

From the number of plants in each class, a disease index (DI) was calculated for each accession as follows:

$$
D I=\frac{\left(1 \times N_{1}\right)+\left(2 \times N_{2}\right)+\left(3 \times N_{3}\right)+\left(4 \times N_{4}\right)}{\left(N_{1}+N_{2}+N_{3}+N_{4}\right)}
$$

where $\mathrm{N}_{\mathrm{n}}$ is the number of plants in the respective class.

\section{Quantification of $V$. longisporum by ELISA}

For quantification of $V$. longisporum biomass in the plant tissue, a direct DAS-ELISA (double antibody sandwich ELISA, Clark \& Adams, 1977) which had been developed in the Institute of Plant Pathology and Plant Protection, University of Goettingen (Cernusko, 1995; Cernusko \& Wolf, 1997) was used comprising biotinylized antibodies and their detection with a streptavidin alkaline phosphatase conjugate (Tian et al., 2005).

Samples, each consisting of five stubbles, were washed under tap water to remove adhesive soil fragments and were divided into roots and shoots (lower $20 \mathrm{~cm}$ of the stem). Samples were air-dried and ground to a fine powder. One g of ground material was mixed with 
extraction buffer (PBS (phosphate buffered saline, $\mathrm{pH}=7.2$ ) $+0.05 \%$ Tween $20+0.2 \%$ PVP (Polyvinylpyrrolidone), 1:20, w/v) and rotated at $4^{\circ} \mathrm{C}$ and $120 \mathrm{RPM}$ over night. One $\mathrm{mL}$ of each extract was transferred to a reaction tube and centrifuged for $10 \mathrm{~min}$ at $13000 \mathrm{xg}$. The supernatant was used as probe for ELISA analyses.

Wells of microtiterplates (Nunc, Wiesbaden, Germany) were filled with $100 \mu \mathrm{L}$ coating buffer and serum-antibodies (1:1000) and incubated at $4^{\circ} \mathrm{C}$ over night. After washing the plates three times with washing buffer for 3 min each, free binding sites were blocked by adding $200 \mu \mathrm{L}$ of blocking buffer $+0.2 \%$ BSA, followed by an incubation of one hour at $37^{\circ} \mathrm{C}$. After another washing step, $100 \mu \mathrm{L}$ samples and protein standards were filled into the wells and incubated for four hours at $37^{\circ} \mathrm{C}$. Again plates were washed three times with washing buffer. Subsequently, $100 \mu \mathrm{L}$ of biotinylized antibodies in PBS/Tween (1:2000) were added and incubated at $4^{\circ} \mathrm{C}$ over night. After three further washing steps $100 \mu \mathrm{L}$ streptavidin alkaline phosphatase $\left(1: 10000\right.$, in PBS/Tween) were added and incubated at $37^{\circ} \mathrm{C}$ for one hour, followed by another washing step. Then, $100 \mu \mathrm{L}$ substrate $(1 \mathrm{mg}$ p-nitrophenyl phosphate per mL substrate buffer) was added and incubated at room temperature in the dark. Extinction was measured after 1,2 and 3 hours at a wavelength of $405 \mathrm{~nm}$ and a reference wavelength of $592 \mathrm{~nm}$ on a plate reader photometer (Spectra 2, SLT Laboratories, Crailsheim). Each sample was measured in two replicates.

\section{Statistical analyses of data}

Data obtained in the greenhouse screening and in scorings of field samples were statistically analyzed using the software package StatGraphics. Differences among means were tested using Fisher's least significant difference (LSD) and considered significant at $p \leq 0.05$. A general linear model procedure was used to identify accessions with a significantly lower $\mathrm{AUDPC}_{\text {norm. }}$ value than the cultivar 'Express', which shows a relatively high tolerance to $V$.

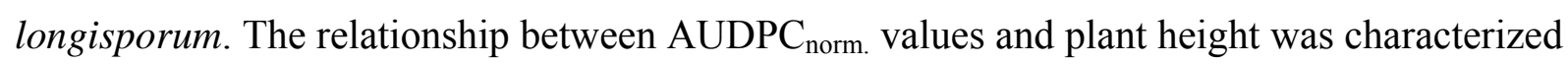
by analysis of correlation. Significance of regression lines was tested with the Durbin-Watson test. Linear regression analysis was also used to determine the relationship between disease severity values of field samples and the corresponding phenotype data from greenhouse experiments and the relationship between disease levels assessed via visual scoring and the extent of colonization by $V$. longisporum, measured by ELISA. 

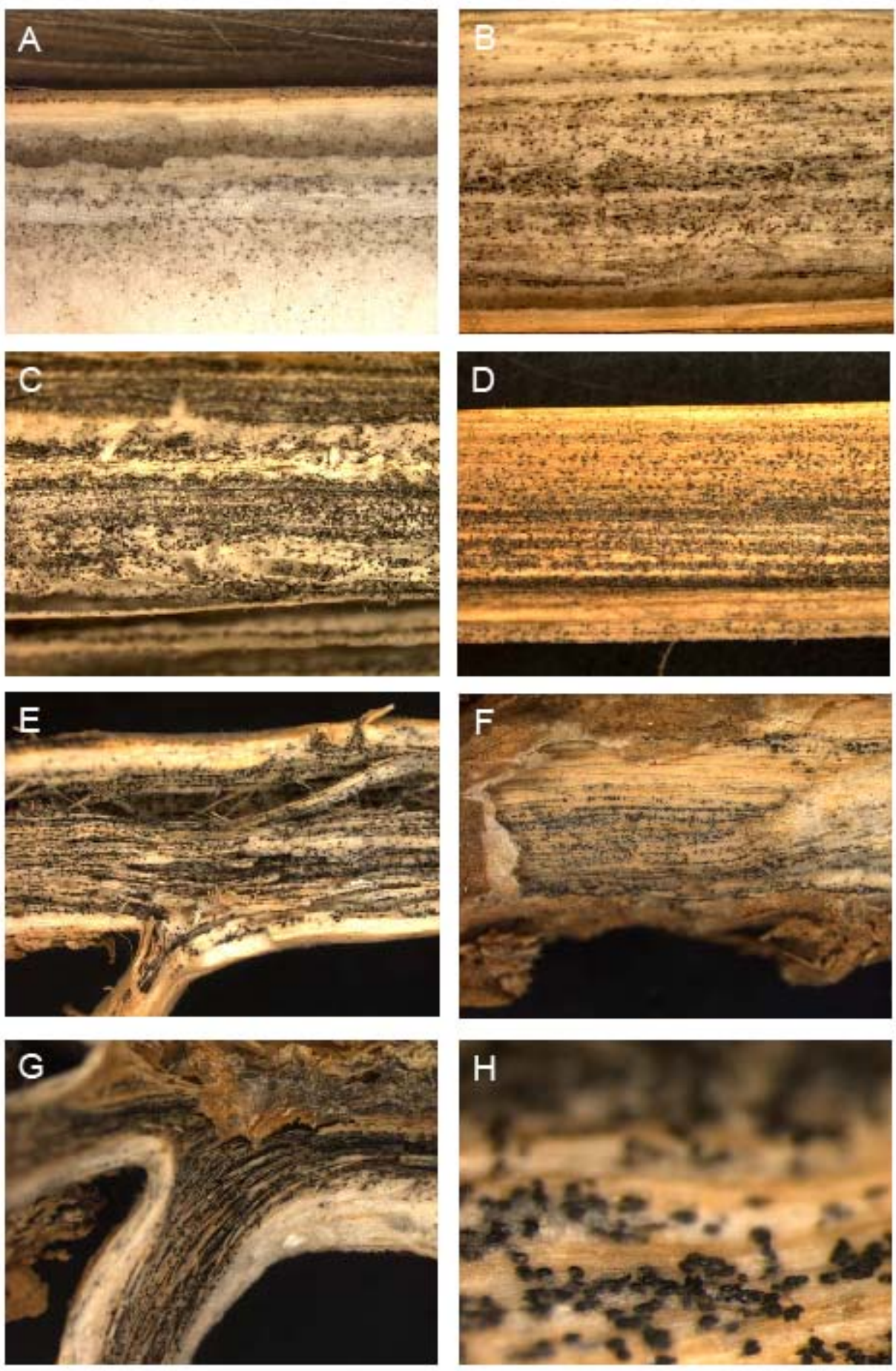

Fig. 2.1. Microsclerotia of $V$. longisporum, produced in the stem pith $(\mathrm{A}-\mathrm{C})$, under the stem epidermis (D), in root tissue (E, G) and on the root surface $(F, H)$. Samples on micrographs A to $\mathrm{C}$ represent scores 2 (A), 3 (B) and 4 (C). 


\section{Results}

Greenhouse screening

Symptoms observed were typical asymmetric yellowing of leaves and the occurrence of black veins (measured as AUDPC value) as well as stunted growth. Plants of the control variant were also scored in order to take into account the unspecific symptoms occurring during the natural ageing process which varied between accessions.

In total, 1348 Brassica accessions comprising genotypes belonging to the group of B. napus, B. rapa, B. oleracea and a small group of undefined varieties were screened for their resistance to $V$. longisporum in 26 independent screening runs over more than 2 years.

There was a large variation in responses to inoculation with $V$. longisporum among the screened accessions. Especially the B. napus genotypes showed a broad range of resistance with $\mathrm{AUDPC}_{\text {norm. }}$ values between 0.02 and 3.21 (Fig. 2.2.). The $\mathrm{AUDPC}_{\text {norm. }}$ value of the cultivars 'Express' and 'Falcon' was 0.76 and 1.26, respectively. A total of 363 B. napus accessions $(29.5 \%)$ possessed AUDPC $_{\text {norm. }}$ values significantly lower $(p \leq 0.05)$ than the tolerant standard 'Express' (Table 2.3).

A similar variation was observed among the progenitor species. Among the B. oleracea accessions, many genotypes showed strong resistance. Thus, with the exception of five, all accessions which had been characterized as resistant to $V$. longisporum in preliminary investigations (Happstadius et al., 2003) also showed an elevated level of resistance in our studies. Additionally, two new resistant B. oleracea genotypes were identified, namely accession 1428 (white cabbage 'Kashirka 202') and accession 2070 (white cabbage 'Ladozhskaya'), listed in Table 2.4. In total, 28 or $84.9 \%$ of the 33 B. oleracea accessions tested had an $\mathrm{AUDPC}_{\text {norm. }}$ value significantly lower than that of 'Express'. 


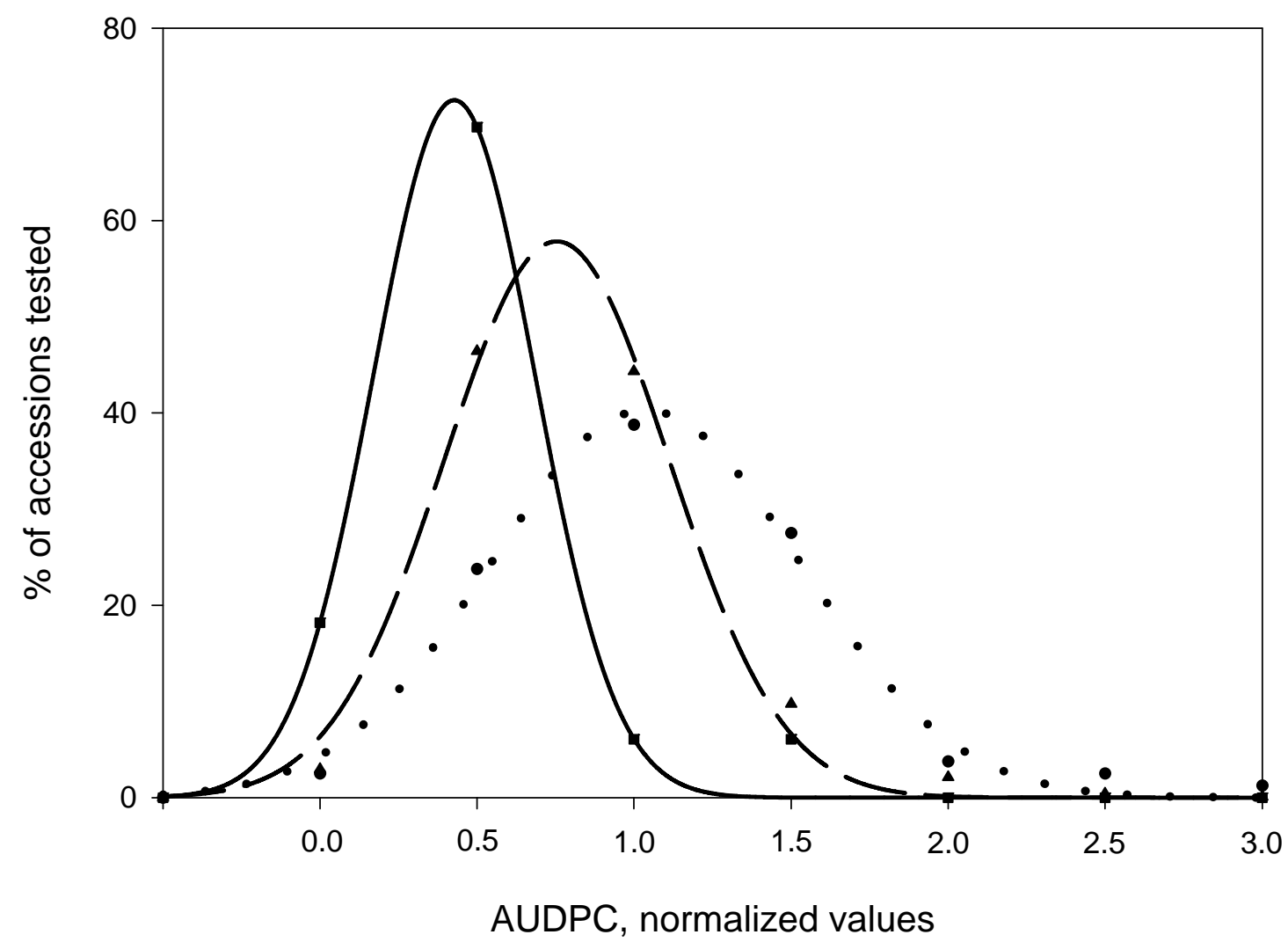

Fig. 2.2: Distribution of resistance responses in the tested Brassica accessions inoculated with $V$. longisporum in 26 independent trials. The frequency distribution of the $\mathrm{AUDPC}_{\text {norm. }}$ values of $B$. oleracea accessions is shown as solid line, that of $B$. napus accessions as dashed line and that of B. rapa accessions as dotted line. The winter oilseed rape cultivars 'Express' $\left(\mathrm{AUDPC}_{\text {norm. }}=0.76\right)$ and 'Falcon' $\left(\mathrm{AUDPC}_{n o r m}=1.26\right)$ served as reference varieties. AUDPC values were calculated based on a rating scale consisting of nine classes and normalized for variation $\left(\mathrm{AUDPC}_{\text {norm. }}\right)$.

Tab. 2.3. Number of Brassica accessions used in this study and the respective percentage of accessions which had an $\mathrm{AUDPC}_{\text {norm. }}$ value significantly lower $(p \leq 0.05)$ than that of the tolerant cultivar 'Express' $\left(\mathrm{AUDPC}_{\text {norm. }}=0.76\right)$.

\begin{tabular}{l|c|c}
\hline Species & No. of tested accessions & \% sign. better than 'Express' \\
\hline B. rapa & 80 & 12.5 \\
B. oleracea & 33 & 84.9 \\
B. napus & 1230 & 29.5 \\
not defined & 13 & 0.0 \\
\hline
\end{tabular}

In contrast to the comparatively high level of resistance in the $B$. oleracea assortment, the $B$. rapa accessions showed a predominantly susceptible or moderately tolerant resistance phenotype. Thus, only $12.5 \%$ of the accessions screened showed a level of resistance which 
was significantly higher than that of the tolerant standard. Particularly the accessions 13444 and G454 'Granat' exhibited moderate resistance phenotypes (Table 2.4). Some of the accessions tested here (B. oleracea accessions 8207, BRA 1398, BRA 1008, 7518, CGN14044; B. rapa accessions 13444, 56515) were used as parental lines for interspecific crossings to create resynthesized oilseed rape plants with improved resistance to $V$. longisporum (Rygulla et al., 2007a, b).

Tab. 2.4. Accession numbers and sources of B. oleracea and B. rapa gene bank accessions and accessions from commercial breeding companies phenotyped for resistance to $V$. longisporum. All accessions listed below showed a resistance level significantly higher than 'Express'.

\begin{tabular}{|c|c|c|c|c|}
\hline Species & Subspecies & Accession & Name & Source \\
\hline B. oleracea & botrytis botrytis & 372897 & Venus & GI \\
\hline B. oleracea & capitata & 1428 & $\begin{array}{l}\text { White cabbage } \\
\text { 'Kashirka 202' }\end{array}$ & NIV \\
\hline B. oleracea & capitata & 2070 & $\begin{array}{l}\text { White cabbage } \\
\text { 'Ladozhskaya' }\end{array}$ & NIV \\
\hline B. oleracea & botrytis botrytis & 3-01 oror & HK Pl. 4/G6 & GI \\
\hline B. oleracea & acephala & 8207 & Kale & HRI \\
\hline B. oleracea & acephala & 5085 & Tainan & HRI \\
\hline B. oleracea & alboglabra & 14044 & Golden & $\mathrm{CGN}$ \\
\hline B. oleracea & alboglabra & 6704 & Chinese Kale & HRI \\
\hline B. oleracea & alboglabra & 6206 & Kainan & HRI \\
\hline B. oleracea & botrytis botrytis & BRA 1398 & Maximus (CSFR) & IPK \\
\hline B. oleracea & botrytis botrytis & 4250 & Kangaroo & HRI \\
\hline B. oleracea & capitata & 5394 & Cappuccio Grosso & HRI \\
\hline B. oleracea & capitata & 10590 & Spring Glory & HRI \\
\hline B. oleracea & capitata & 7096 & Filderkraut & $\mathrm{CGN}$ \\
\hline B. oleracea & capitata capitata & BRA 504 & De Buzan & IPK \\
\hline B. oleracea & capitata capitata & BRA 544 & $\begin{array}{l}\text { Kodanske Trzni Rane } \\
\text { (CSFR) }\end{array}$ & IPK \\
\hline B. oleracea & capitata capitata & BRA 723 & $\begin{array}{l}\text { Wiener Dauer } \\
\text { Weißkraut }\end{array}$ & IPK \\
\hline B. oleracea & capitata & 3775 & $\begin{array}{l}\text { Golden Acre Early } \\
\text { Ironside }\end{array}$ & HRI \\
\hline B. oleracea & capitata & 4622 & Cabbage (?) & HRI \\
\hline B. oleracea & costata & 7799 & Couve Murciana & HRI \\
\hline B. oleracea & gemmifera & 2808 & $\begin{array}{l}\text { Brussels Sprout, } \\
\text { Lenteweelde }\end{array}$ & HRI \\
\hline B. oleracea & gemmifera & 10618 & Brussels Sprout, Tiara & HRI \\
\hline B. oleracea & botrytis italica & BRA 1427 & Brokkoli (Irak) & IPK \\
\hline
\end{tabular}




\begin{tabular}{|c|c|c|c|c|}
\hline Species & Subspecies & Accession & Name & Source \\
\hline B. oleracea & botrytis italica & 7518 & Brokkoli, (PDCTE) & HRI \\
\hline B. oleracea & botrytis italica & 2398 & Brokkoli (PDP) & HRI \\
\hline B. oleracea & botrytis italica & 4707 & Calabrese & HRI \\
\hline B. oleracea & $\begin{array}{l}\text { acephala } \\
\text { sabellica }\end{array}$ & BRA 1008/79 & Grünkohl (DDR) & IPK \\
\hline B. oleracea & sabellica & & Frostara & $\begin{array}{l}\text { Cultivar, } \\
\text { KK }\end{array}$ \\
\hline B. rapa & pekinensis & 13444 & Chinese cabbage & BAZ \\
\hline B. rapa & narinosa & 15407 & unknown & GI \\
\hline B. rapa & pekinensis & G 454 & $\begin{array}{l}\text { Chinese cabbage } \\
\text { 'Granat' }\end{array}$ & $\begin{array}{l}\text { Cultivar, } \\
\text { unknown }\end{array}$ \\
\hline B. rapa & rapa & G 684 & $\begin{array}{l}\text { 'Runde weiße } \\
\text { rotköpfige } \\
\text { Herbstrüben' }\end{array}$ & $\begin{array}{l}\text { Cultivar, } \\
\text { unknown }\end{array}$ \\
\hline B. rapa & oleifera annua & & '00-Sommerrübsen` & NPZ \\
\hline B. rapa & oleifera biennis & Q3F & Q3F & NPZ \\
\hline B. rapa & pekinensis & M241 & $\begin{array}{l}\text { Chinese cabbage 'Pe } \\
\text { Tsai' }\end{array}$ & GI \\
\hline B. rapa & unknown & unknown & unknown & KWS \\
\hline B. rapa & oleifera annua & unknown & Nokonova & $\begin{array}{l}\text { Cultivar, } \\
\text { KWS }\end{array}$ \\
\hline B. rapa & oleifera hiemalis & unknown & Buko & $\begin{array}{l}\text { Cultivar, } \\
\text { KWS }\end{array}$ \\
\hline
\end{tabular}

Abbreviations: BAZ: Gene Bank of the Federal Centre for Breeding Research on Cultivated Plants, Braunschweig, Germany; CGN: Centre for Genetic Resources, Wageningen, Netherlands; GI: Department of Plant Breeding, Research Centre for Biosystems, Land Use and Nutrition, Giessen, Germany; HRI: Horticulture Research International Genetic Resources Unit, Warwick, UK; IPK: Institute of Plant Genetics and Crop Plant Research, Gatersleben, Germany; NIV: Genebank of the NI Vavilov Institute of Plant Industry (VIR), St. Petersburg, Russia; NPZ: Norddeutsche Pflanzenzucht Hans-Georg Lembke KG, Hohenlieth, Germany; KK: Bruno Nebelung GmbH \& Co. KG, Kiepenkerl Pflanzenzüchtung, Everswinkel, Germany; PDCTE: Precoce Di Calabria Tipo Esportazione; PDP: Picolini Di Palermo

With the exception of one trial, there was a highly significant negative correlation between $V$. longisporum resistance levels, assessed via calculation of AUDPC values from symptom scores, and the effect of infection on plant height, expressed as normalized plant height, with correlation coefficients ranging from -0.60 to -0.95 . An example is shown in Figure 2.3. Interestingly, a relatively weak significant relationship between $\mathrm{AUDPC}_{\text {norm. }}$ values and the normalized plant height $(\mathrm{r}=-0.40)$ was found in a trial where only rapa accessions were tested for their resistance response. 


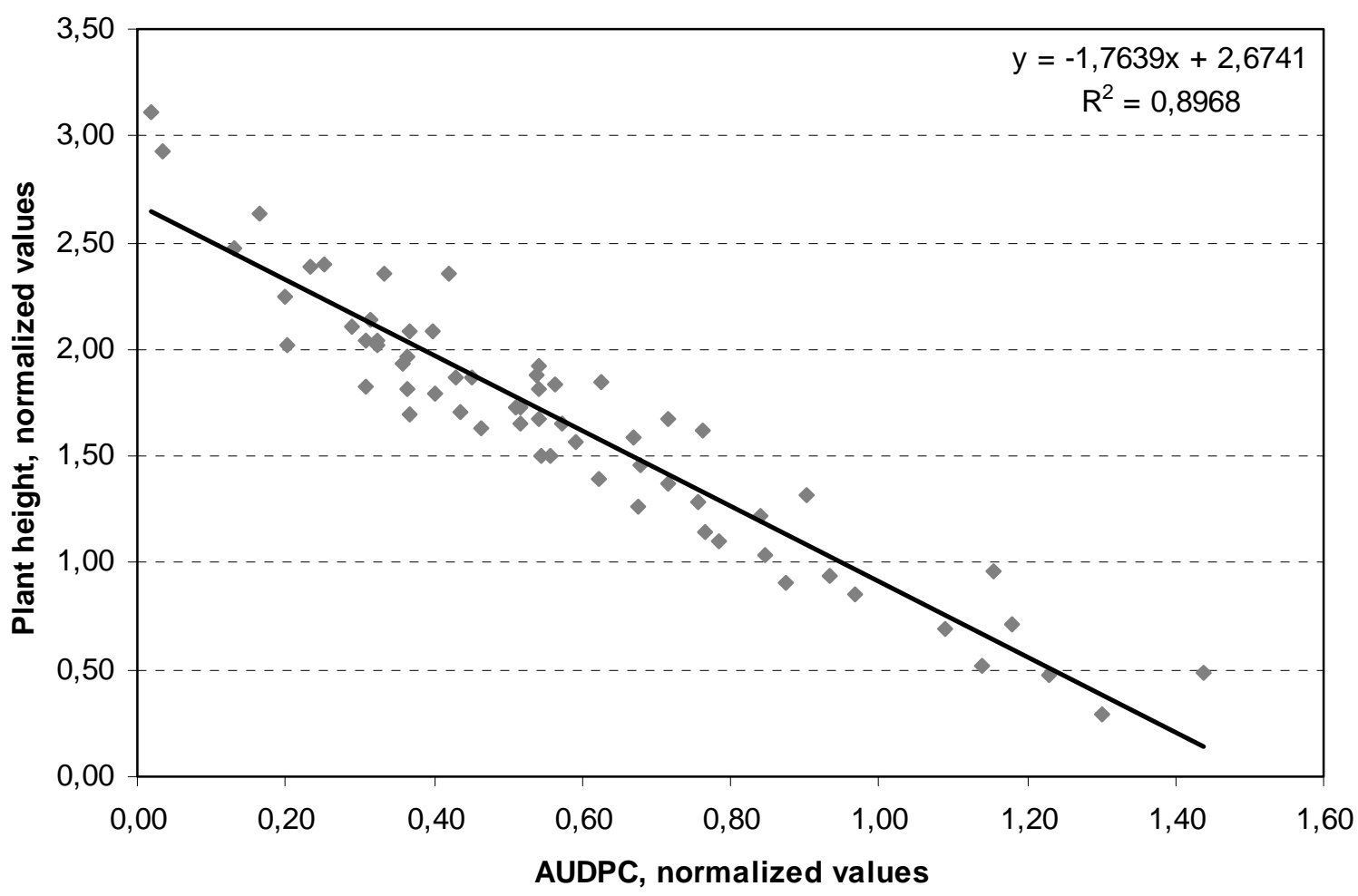

Fig. 2.3. Relationship between $V$. longisporum resistance levels in B. napus accessions, assessed by calculation of AUDPC values from symptom scores, and the effect of an infection on plant height, expressed as normalized plant height. Data of the $22^{\text {nd }}$ screening run are shown, carried out from 13.11. - 21.12 2006.

Field resistance tests

Following the resistance screening in the greenhouse, selected accessions of B. napus were tested under field conditions. After harvest, stubble samples were collected randomly from each plot and scored with regard to the abundance of microsclerotia. Average disease incidence (DI) and disease severity (DS) values were calculated from scores obtained in all three years of field experiments. In parallel, those accessions which were to be evaluated in the field were tested again in one screening run in the greenhouse.

In the year 2004/05, the majority of the eight cultivars tested exhibited a moderate disease level with DI values ranging from 40 to $60 \%$ and DS varying from 1.66 ('Smart') to 2.0 ('Express', 'Mohican',' Talent') on the scale from 0 to 4 . While cultivar 'Elektra' exhibited a very high disease level with a DI of up to $92 \%$ and a DS of 2.12 , cultivar 'Oase' by far showed the lowest infestation level with a DI between 8 and 22\% and a DS of 1.24 (Fig. 2.4). 


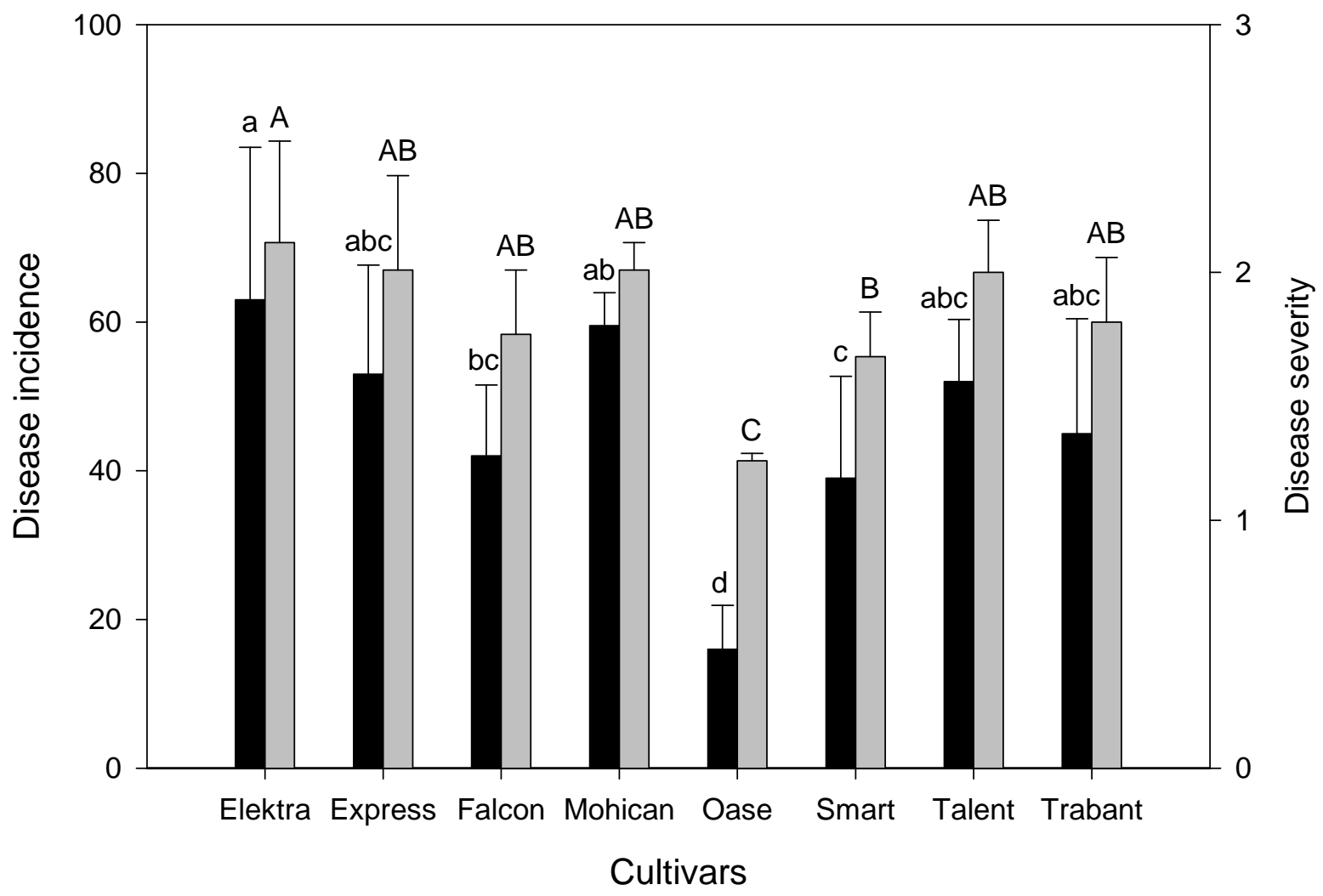

Fig. 2.4. Disease incidence (black) and disease severity (grey) of different winter oilseed rape cultivars on a site that has been artificially inoculated before sowings (Große Lage, Goettingen). Columns and whiskers represent mean values and standard deviations from 50 plants of each cultivar. Data with the same letters do not differ on a significance level of $p \leq$ 0.05 (LSD).

The following season (2005/06) was characterized by a generally low infestation level, with the exception of the site at Fehmarn, a site known for its long tradition of oilseed rape growing. Because the tested accessions showed only poor differentiation with regard to the disease severity levels, only the disease incidence values will be considered in the following part.

The locations Futterkamp, Thuele and Goettingen exhibited DI levels ranging from 0 to $40 \%$. By contrast, the DI reached values of up to $94 \%$ in Fehmarn. The reference varieties possessed DI values as expected from the results of previous greenhouse tests with the exception of cultivar 'Laser' which showed a low infestation level in Futterkamp and cultivar 'Lion' possessing a relatively high DI level in Thuele. Most of the five accessions (SEM 1,

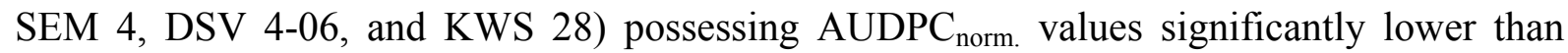
'Express' under standardized conditions also showed comparatively low disease levels in the 
field. However, at each site the other of these promising accessions, with the exception of SW 1, exhibited DI values unexpectedly high (Table 2.6).

In 2006/07 the incidence of $V$. longisporum in Thuele was nearly zero. Hence, this site was not included in further analyses. Contrary to the previous year, accessions showed a good differentiation with regard to disease severity. In Goettingen, the infestation turned out to be on a low to moderate level with a DI varying from 4 to $64 \%$ and DS values ranging from 1.08 to 2.31 . While the disease level was already fairly high in Rostock (DI: 20 - 94\%, DS: $1.16-$ 2.52) the degree of infestation revealed to be extremely high in Fehmarn with the DI being $86 \%$ at minimum and reaching $100 \%$ in six accessions. Accordingly, the disease severity ranged from 1.93 to 3.05. Similar to observations in 2005/06, some of the accessions which performed extremely well in the greenhouse test also showed enhanced resistance levels in the field, however this coherence was less pronounced under the high disease pressure in Fehmarn. On the other hand, some accessions exhibited low disease levels in all three field experiments but not under greenhouse conditions, namely accessions SEM 05019, SEM 05027 and DM 120.05 as well as the reference variety 'Oase'. Others (KWS 66, SRG 2, SRG 1, and NPZ 4) did exhibit high resistance at sites with lower infestation levels, such as Goettingen and Rostock, but did not so in Fehmarn or in the greenhouse (Table 2.7).

Comparison of results from resistance tests in the greenhouse and in the field

Regression analyses of phenotype data obtained in greenhouse experiments and disease levels determined in the field showed no statistically significant relationship, neither between greenhouse and field data nor between results of different field sites. The coefficient of determination ranged from 0.0002 to 0.1529 in the season 2005/06 and 0.0012 to 0.1243 in 2006/07. Only regression analyses between data from Goettingen and Fehmarn in 2006/07 showed a slightly positive correlation with $r=0.55$. The possible reasons for these findings will be discussed later. 


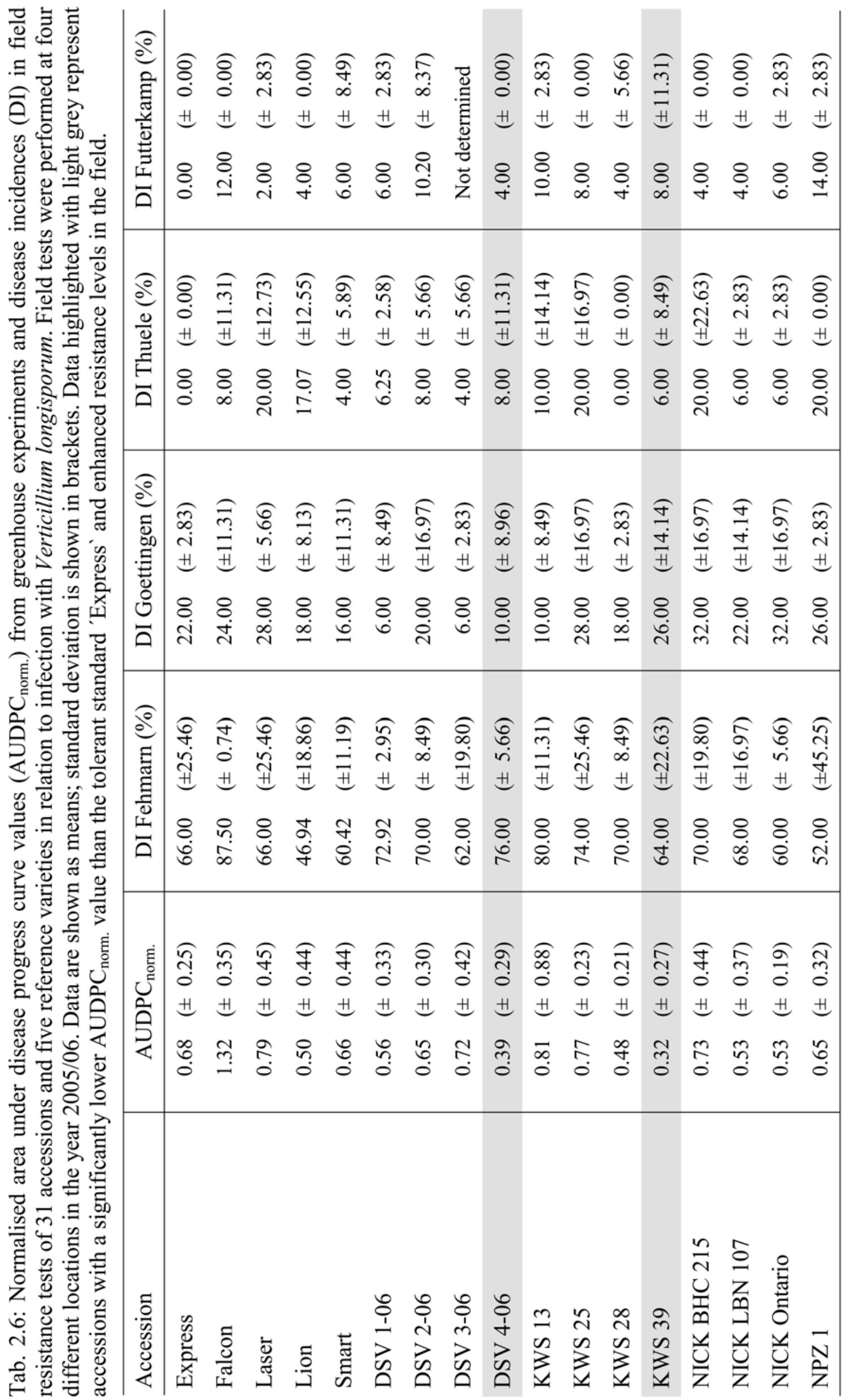




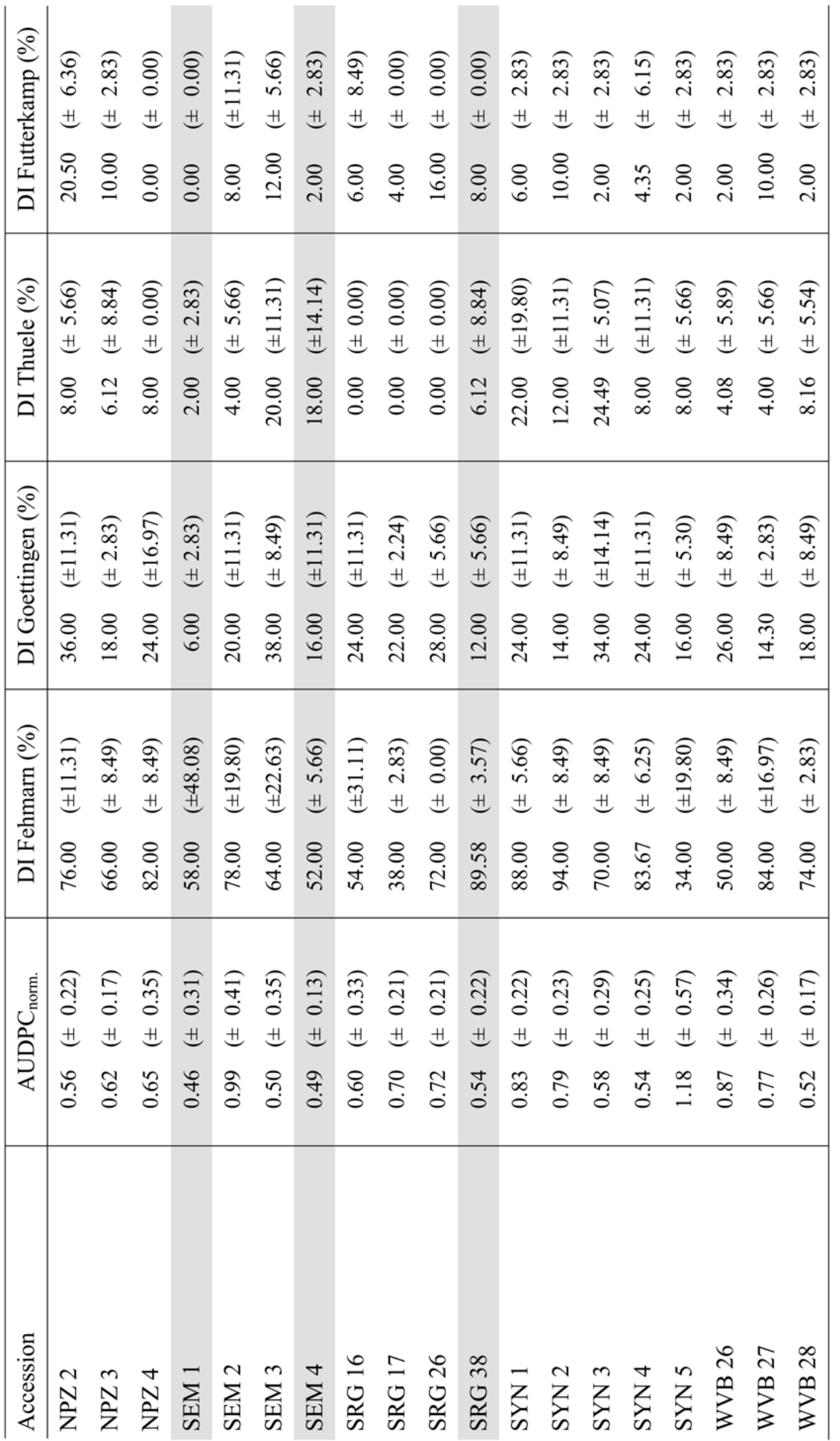




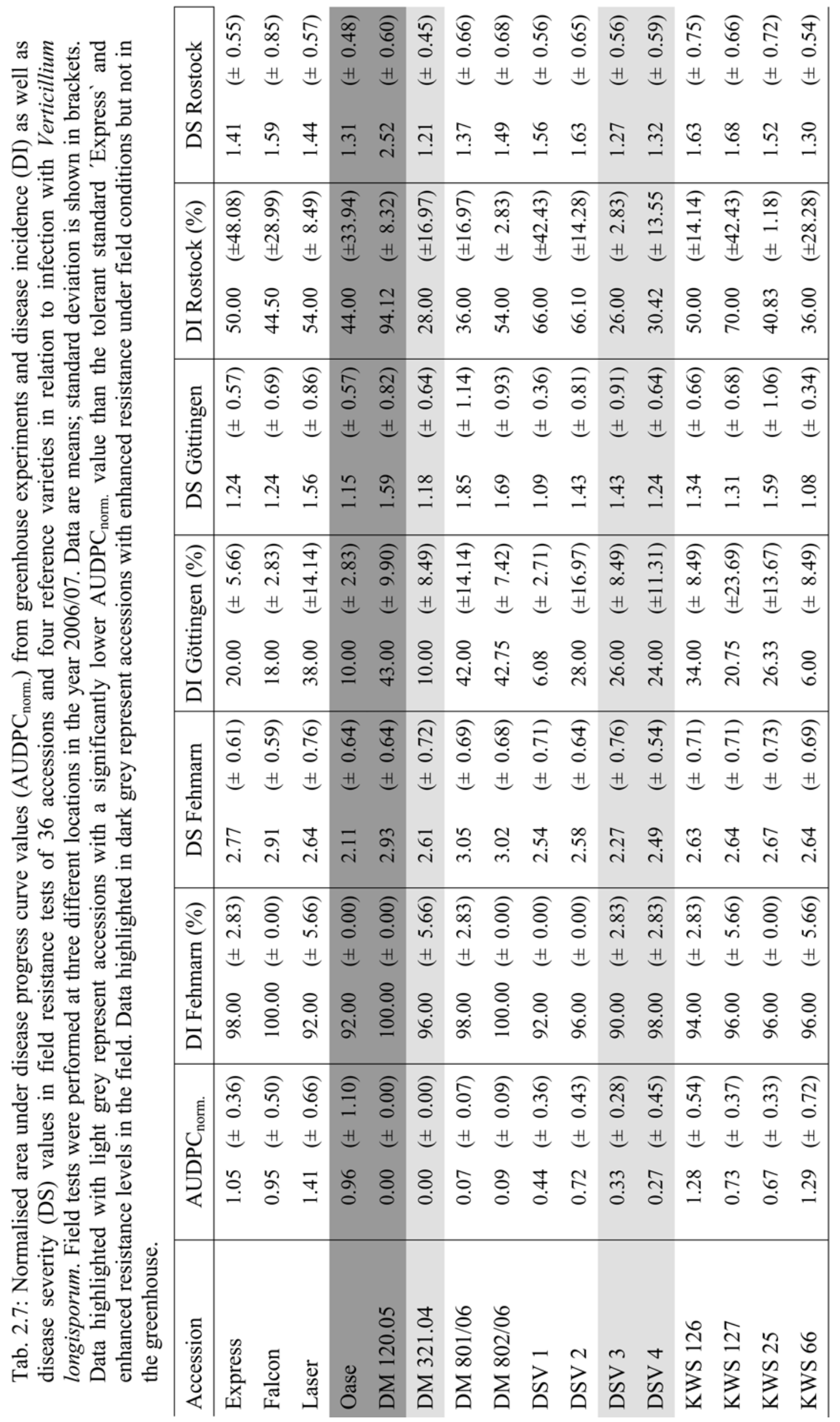




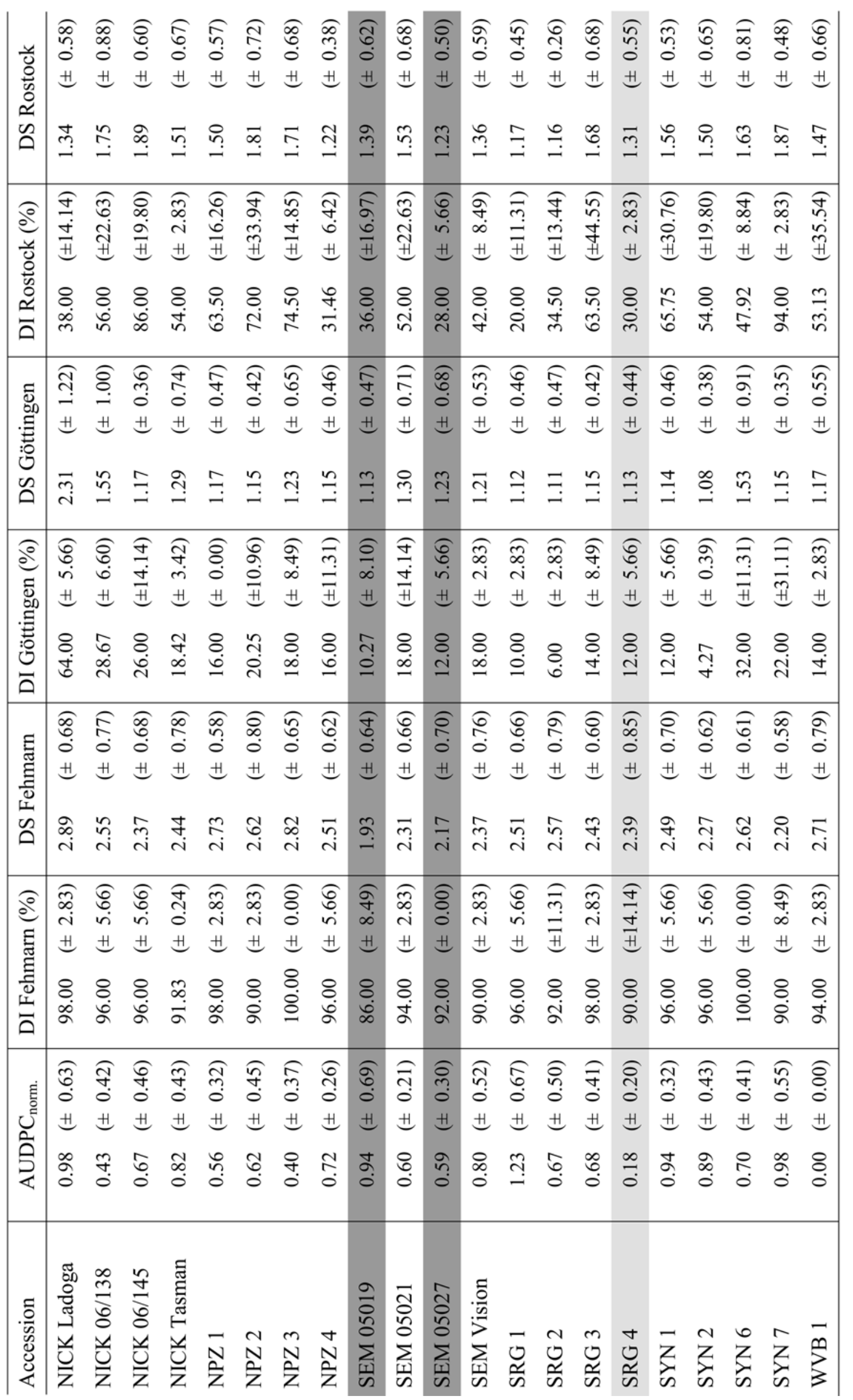




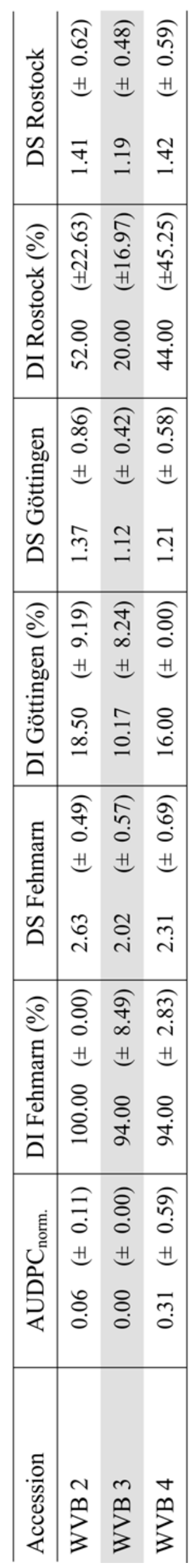


Disease development in the field

The occurrence and systemic spread of $V$. longisporum in different cultivars and accessions after artificial soil infestation was followed with ELISA analysis of samples taken in the course of the vegetation period during two years of field experiments (2004-2006).

In the year 2004/05, in samples taken between autumn and the next following June no Verticillium antigens were detected. It was not before July (GS 89) that the first verifiable amounts of the fungus were found in both root and shoot tissues. Four weeks later, at harvest time, substantial colonization, particularly of the shoots, was observed. Cultivar 'Oase' which showed by far the lowest disease level with respect to incidence and severity, also possessed the lowest amounts of Verticillium protein in the shoots. However, this was not confirmed statistically in comparison to cultivars 'Talent', 'Trabant', 'Falcon', and 'Elektra' (Figure $2.5)$.

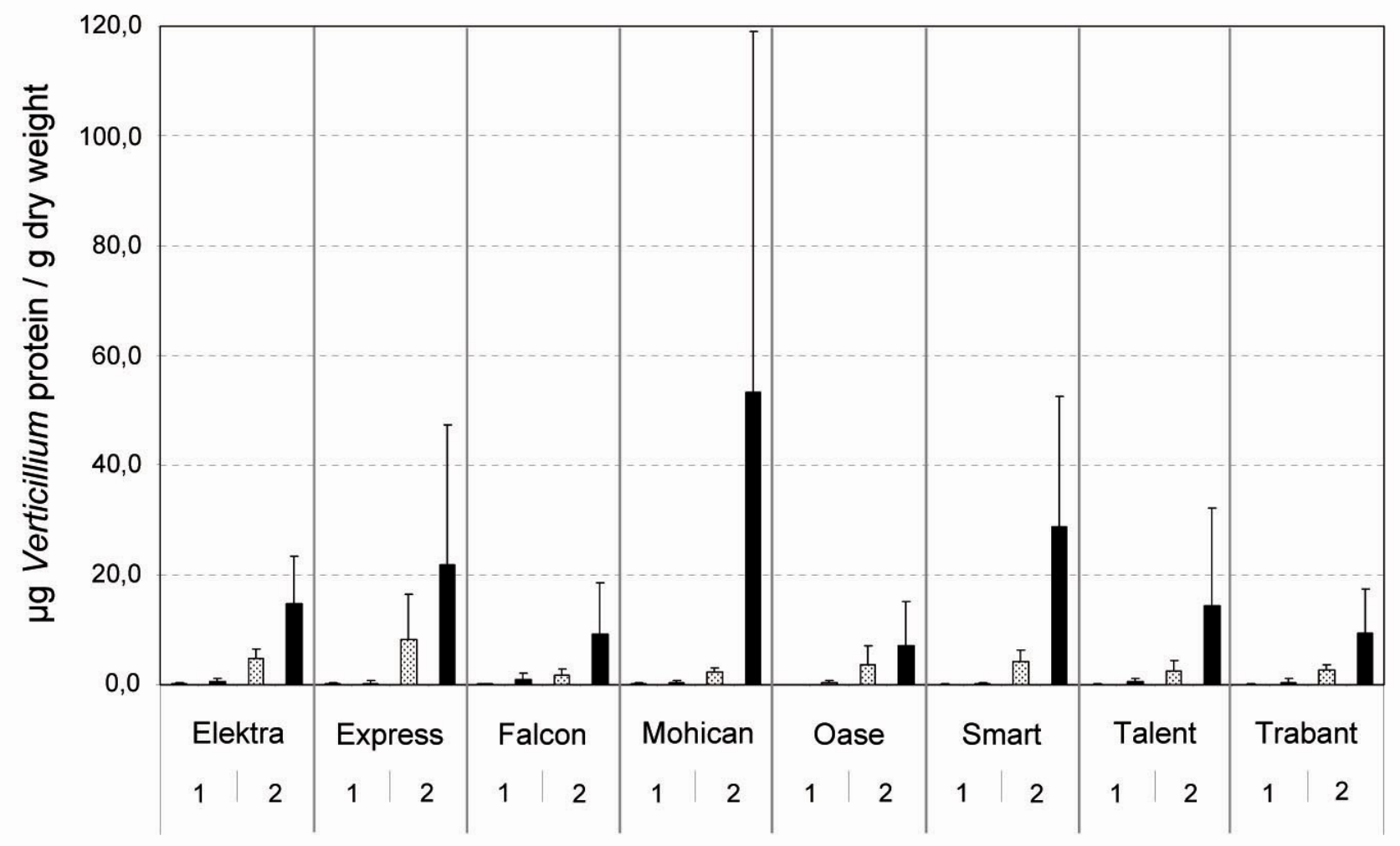

Cultivars

Fig. 2.5. Colonization of roots (dotted bars) and shoots (black bars) of different winter oilseed rape cultivars by $V$. longisporum as detected by ELISA in plant samples from the field taken in July and August 2005 after artificial infestation of the soil before sowing in 2004. Sampling time point (STP) $1=14^{\text {th }}$ July (GS 89), STP $2=2^{\text {nd }}$ August (harvest). Columns and whiskers represent the mean and standard deviations of five samples of two repetitions each; the experiment was conducted in Goettingen, Germany. 
In line with the low infestation level determined by visual scorings in the season 2005/06, substantial amounts of $V$. longisporum in root and shoot tissues were not detected until harvest ( $1^{\text {st }}$ August, GS 99). The amount of fungal protein in the shoots ranged from $0.02 \mu \mathrm{g} /$ g dry weight (dw; DSV 2-06) to $374.43 \mu \mathrm{g} / \mathrm{g}$ dw (NPZ 2). In the roots values reached up to $66.62 \mu \mathrm{g} / \mathrm{g} \mathrm{dw}$ (SRG 16). Accessions which showed a mean disease incidence below 10\% (DSV 1-06, DSV 3-06, DSV 4-06, KWS 13, SEM 1) at this site (Goettingen) also exhibited a limited colonization by the fungus with fungal protein amounts less than $10 \mu \mathrm{g} / \mathrm{g} \mathrm{dw}$; however, regression analyses comprising all accessions tested did not show a statistically significant relationship between disease incidence values assessed via visual scorings and the extent of colonization measured by ELISA after harvest (Table 2.5).

Tab. 2.5. Colonization of roots and shoots of different winter oilseed rape accessions by $V$. longisporum as detected by ELISA in plant samples collected at harvest time in summer 2006 from a field artificially infested before sowings in 2005. Data represent means of five samples from two replicates each. Standard deviation is shown in brackets. The experiment was conducted on an experimental field in Goettingen, Germany. Accessions with a disease incidence level below 10\% are highlighted in light grey.

\begin{tabular}{l|c|c}
\hline \multirow{2}{*}{ Accession } & \multicolumn{2}{|c}{$\mu \mathrm{g}$ protein $/ \mathrm{g}$ dry weight } \\
\hline DSV 2-06 & $0.02( \pm 0.02)$ & Root tissue \\
Lion & $0.02( \pm 0.00)$ & $0.03( \pm 0.01)$ \\
DSV 3-06 & $0.33( \pm 0.46)$ & $0.03( \pm 0.00)$ \\
NICK Ontario & $0.45( \pm 0.58)$ & $2.56( \pm 3.59)$ \\
KWS 13 & $0.67( \pm 0.88)$ & $0.03( \pm 0.01)$ \\
Smart & $2.17( \pm 3.07)$ & $0.05( \pm 0.06)$ \\
Express & $2.27( \pm 3.21)$ & $1.18( \pm 1.49)$ \\
WVB 27 & $3.78( \pm 5.34)$ & $1.07( \pm 1.49)$ \\
DSV 1-06 & $3.88( \pm 5.47)$ & $4.16( \pm 2.08)$ \\
DSV 4-06 & $4.46( \pm 6.18)$ & $3.17( \pm 4.45)$ \\
KWS 25 & $4.71( \pm 5.55)$ & $8.38( \pm 11.64)$ \\
SYN 2 & $4.79( \pm 6.71)$ & $8.78( \pm 3.76)$ \\
NPZ 4 & $4.93( \pm 6.97)$ & $0.85( \pm 0.50)$ \\
SEM 1 & $5.28( \pm 7.19)$ & $7.31( \pm 10.29)$ \\
Falcon & $5.58( \pm 7.77)$ & $2.77( \pm 3.77)$ \\
SEM 3 & $6.04( \pm 2.06)$ & $2.88( \pm 4.07)$ \\
KWS 39 & $8.47( \pm 11.73)$ & $2.73( \pm 2.40)$ \\
\hline & & $1.42( \pm 1.75)$ \\
\hline
\end{tabular}




\begin{tabular}{l|c|c}
\hline \multirow{2}{*}{ Accession } & \multicolumn{2}{|c}{$\mu \mathrm{g}$ protein / g dry weight } \\
\hline WVB 26 & Shoot tissue & Root tissue \\
NPZ 1 & $11.86( \pm 6.51)$ & $39.08( \pm 51.19)$ \\
SRG 38 & $14.94( \pm 10.57)$ & $5.50( \pm 7.54)$ \\
NPZ 3 & $22.16( \pm 28.48)$ & $3.83( \pm 1.16)$ \\
SRG 26 & $23.52( \pm 32.62)$ & $7.13( \pm 5.93)$ \\
SYN 5 & $24.67( \pm 30.19)$ & $4.99( \pm 6.94)$ \\
SYN 4 & $26.16( \pm 24.69)$ & $28.94( \pm 36.18)$ \\
WVB 28 & $31.53( \pm 23.15)$ & $32.55( \pm 42.74)$ \\
SEM 2 & $31.87( \pm 29.73)$ & $5.01( \pm 1.83)$ \\
Laser & $33.57( \pm 03.08)$ & $22.90( \pm 28.00)$ \\
NICK LBN 107 & $37.38( \pm 44.96)$ & $6.53( \pm 0.32)$ \\
SEM 4 & $41.25( \pm 58.34)$ & $19.43( \pm 19.63)$ \\
KWS 28 & $46.18( \pm 65.30)$ & $7.70( \pm 10.66)$ \\
SYN 3 & $54.74( \pm 32.95)$ & $7.39( \pm 10.36)$ \\
SRG 17 & $71.34( \pm 85.36)$ & $14.38( \pm 9.34)$ \\
SRG 16 & $167.17( \pm 210.19)$ & $23.12( \pm 7.18)$ \\
SYN 1 & $179.50( \pm 253.83)$ & $66.62( \pm 26.54)$ \\
NPZ 2 & $374.43( \pm 373.81)$ & $8.98( \pm 12.46)$ \\
\hline A & & $21.44( \pm 19.12)$ \\
\hline
\end{tabular}

Abbreviations: DSV: Deutsche Saatveredelung Lippstadt Bremen GmbH, KWS: Klein Wanzlebener Saat AG, NICK: Limagrain Nickerson GmbH, SEM: Svalöf Weibull Seed Hadmersleben GmbH, SYN: Syngenta Seeds GmbH, SRG: Raps GbR Saatzucht Lundsgaard, NPZ: Norddeutsche Pflanzenzucht Hans-Georg Lembke KG, WVB: W. von BorriesEckendorf $\mathrm{GmbH} \& \mathrm{Co}$.

\section{Discussion}

Great efforts are being made to develop oilseed rape cultivars with total or at least partial resistance to $V$. longisporum. For this purpose large populations of plant accessions and breeding progenies need to be screened for resistance. Evaluation of resistance response is usually performed either by dipping roots of young plants into a conidial suspension, followed by replanting in soil (Zeise, 1992; Koike et al., 1994; Subbarao et al., 1995; Zeise \& Buchmüller, 1997; Steventon et al., 2002b; Happstadius et al., 2003; Debode et al., 2005, Dunker et al., 2006), or by sowing the seeds directly in soil that has been artificially infested with microsclerotia (Debode et al., 2005; Dunker et al., 2006). Most greenhouse resistance 
tests described are based on a method developed by Koike et al. (1994) who compared different inoculation methods and described the root-dip method as producing the most consistent results in repeated experiments. However, this screening procedure is time consuming, plants have to be grown from 14 (Happstadius et al., 2003) to 30 days (Koike et al., 1994; Subbarao et al., 1995) before inoculation and scoring may not be performed until four (Koike et al., 1994), six (Subbarao et al., 1995), seven (Debode et al., 2005) or even ten weeks (Happstadius et al., 2003) after inoculation.

Zeise (1992) developed a screening assay where seven to ten days-old plants were inoculated, with a disease evaluation 28 days post inoculation. This screening method provided consistent results in independent trials and is at least three weeks faster than the other assays listed above, therefore the greenhouse screening in this study followed the method by Zeise.

Steventon et al. (2002b) developed an in vitro test, the so-called 'box test method', in order to provide a rapid and reliable evaluation of the susceptibility of cultivars of B. napus to Verticillium wilt. Besides being in good accordance with the root-dipping method when run in parallel, the in vitro test was 3.5 times faster than the original root-dip method which did not allow for a disease rating until 12 weeks after sowing (Koike et al., 1994). Thus, disease rating was already possible at $25 \mathrm{dpi}$. An adaptation of this method is described by Debode et al. (2005). The 'box-test' method does not have a distinct advantage over the Zeise method with regard to the time required. However, it would have been impracticable to screen such high numbers of accessions as was necessary in our studies (60 to 70 genotypes per run) under sterile conditions. This again made the method developed by Zeise the preferable one for the purposes of this work. However, because the root dip inoculation method requires root damage and the use of high spore inoculum (Heale, 2000) this method a priori does not allow to differentiate for resistance mechanisms at the level of root penetration, since the pathogen may invade the plant xylem by wounded roots. However, according to Pegg \& Brady (2002) who reviewed the comprehensive body of literature on vascular wilts, there are no reports on total resistance to infection. Thus, plant/Verticillium spp. pathosystems with monogenic and/or polygenic resistance obviously share the general feature to allow at least limited fungal root or hypocotyl colonization, regardless whether the roots have been damaged before infection or not. While wounds are not essential for invasion by $V$. longisporum and most other vascular pathogens, the provision of wounded roots in the screening assay at least improves the uniformity of disease development and may thus be equally important as a controlled environment during the screening procedure (Walker, 1965). 
By using the root dip inoculation method the initial phases of the disease can be recorded and highly susceptible genotypes can be eliminated at an early stage. On the other hand, resistant plants can be vernalized and then be allowed to continue to grow under greenhouse conditions for evaluation as mature plants. This also allows for the development of seeds for progeny tests. This approach is of particular interest if only a limited number of seeds is available, for example during the evaluation of newly resynthesised B. napus genotypes in which resistance to $V$. longisporum has been introduced from B. oleracea, as was done with the help of some of the accessions tested in this study within the scope of a project cooperation with the Institute of Plant Breeding of the Justus-Liebig-University of Giessen and the Association of German Plant Breeders e.V. (GFP; Rygulla et al., 2007b), and also in a study of Happstadius et al. (2003).

Brassica species within the gene pool of B. napus, B. oleracea and B. rapa show a large variation in resistance to $V$. longisporum. Using a mixture of isolates VL 40 and VL 43, a severe disease progression was induced in most B. napus and B. rapa accessions. Thus, after two weeks, infected plants showed yellowing of leaves, the occurrence of black veins and a distinct growth reduction. Particularly in the B. rapa germplasm very few genotypes $(12.5 \%)$ exhibited a resistance level significantly higher than that of the relatively tolerant reference cultivar 'Express' (Happstadius et al., 2003). This latter finding is confirmed by results of Yui et al (1985) who, by testing the resistance to Verticillium of several B. rapa accessions, demonstrated that most of the genotypes were highly susceptible and only a few cultivars of the Kanamachi varietal group of B. campestris var. rapifera showed almost no symptoms. In the present studies, resistance screening of $B$. rapa accessions resulted in the identification of two accessions (13444 and G545) of the cultivar group pekinensis with moderate resistance to V. longisporum (see also Rygulla et al., 2007a).

By comparing inoculated plants of B. napus cultivar 'Express' with a collection of 33 cabbage accessions, several accessions with a high level of resistance to Verticillium were identified, meaning that plants exhibited only faint or even no symptoms after infection. This result is consistent with earlier findings of Happstadius et al. (2003) who tested several B. oleracea and wild species accessions, as did Debode et al. (2005) and Zeise \& Buchmüller (1997) who identified $B$. oleracea and $B$. carinata as the most resistant species when compared with $B$. napus, B. rapa, B. nigra, and B. juncea. Contrary to this, Subbarao (1994) described bok choy, Chinese cabbage, cabbage and especially Brussels sprout to be susceptible to Verticillium. Again in other investigations even highly infested commercial broccoli fields remained unaffected (Koike et al., 1994). These contradictory results probably derive from the 
fact that each group assumably used different $V$. longisporum isolates, potentially from distinct geographical regions and therefore representing special features of their origin. However, according to Happstadius et al. (2003), the Brassica C genome is most likely to contain resistance trait(s) to $V$. longisporum. Thus, resistance of Chinese cabbage (B. oleracea L. var. capitata) is reported to be inherited in a dominant manner mediated by multiple genes (Kemmochi et al., 2000). Further possible sources of resistance to the pathogen are Brassica wild species, like B. cretica, B. incana, B. insularis, and B. villosa. However, until now, no higher levels of resistance have been detected in these ancient varieties (Happstadius et al., 2003).

The infection progress of $V$. longisporum in the field is characterized by an extended latency, as was documented by ELISA. Thus, although it has been shown that infections do occur in autumn (Zeise \& Seidel, 1990), the fungus seems to persevere in the hypocotyl and lower stem part with very little fungal biomass and it is not until the beginning of plant senescence that the fungus spreads systemically and produces substantial biomass, together with the appearance of first visible symptoms. These characteristics of the disease dynamics of $V$. longisporum in winter oilseed rape have already been described by other authors (Wolf $\&$ Weinert, 2003; Steinbach et al., 2005; Dunker et al., 2006). The fact that initiation of flowering is a critical period for disease progression in the Verticillium pathosystem has already been reported for Arabidopsis thaliana (Steventon et al., 2001; Veronese et al., 2003) and Solanum tuberosum. Early maturing potatoes generally showed a higher susceptibility to Verticillium (Nachmias et al., 1990; Jansky \& Rouse, 2000; Simko et al., 2004b). According to these observations the low disease scores of winter oilseed rape cultivar 'Oase' might not be due to real resistance or tolerance but to the late ripening type of this cultivar (Federal Cultivar List of the 'Bundessortenamt', 2007). This assumption is supported by the fact that the cultivar 'Oase' exhibits only moderate resistance under greenhouse conditions (data not shown).

This again shows that results obtained in the greenhouse may not be easily transferred to the field situation. Factors such as the ripening behaviour of particular genotypes, which is not considered in a greenhouse assay, may interfere with mechanisms that govern the resistance response of the plant. In general, the fact that environmental factors commonly influence the rate and degree of disease development is well established. Furthermore, it is recognized by many breeders that the expression of resistance may also be enhanced by one or another external factor to which a breeding population is exposed (Walker, 1965). For example, in early investigations on the effect of environmental conditions on the resistance of cabbage to 
yellows caused by Fusarium oxysporum var. conglutinans (Walker \& Smith, 1930) it has been shown that at a constant soil substrate temperature of $24^{\circ} \mathrm{C}$, all susceptible and multigene-resistant plants became diseased, while those ones homozygous or heterozygous for monogenic resistance survived. If the temperature rose much above $24^{\circ} \mathrm{C}$, even monogene-resistant plants succumbed to root decay and if it was much lower than $24^{\circ} \mathrm{C}$, some multigene-resistant plants survived (Walker, 1963).

In the present studies, regression analysis of the results obtained under greenhouse conditions showed no correlation with field resistance tests of individual experimental sites nor with the average of all locations in both years of field experiments $(r=0.014$ to 0.391$)$. The possible reasons for this are manifold. On the one hand this might be due to an uneven distribution of inoculum at different field sites. This may have been the case in Thuele and Fehmarn with extremely low or high infestation levels in 2006/07, respectively, or even in one field, which in turn might lead to sporadic infection levels. The latter was especially true for single repetitions in Futterkamp and Thuele in 2005/06, leading to an extremely high standard deviation (Table 2.6). Even artificial inoculation, as performed on the Goettingen field site, did not guarantee for homogenous infection. This may be considered a general constraint in field testing against soilborne pathogens and thus the tolerance or susceptibility of an accession might be over- or underestimated.

To our knowledge, only limited investigations have been carried out up to now regarding the occurrence of pathotypes differing through their geographic origin. Both isolates used in the greenhouse test constitute strains which have been isolated from diseased plants in the field and have been proven to be highly aggressive to Brassica species in several tests (Zeise \& von Tiedemann, 2002a, b). A varying degree in aggressiveness of particular strains due to the geographic origin may additionally interfere with the field testing of different genotypes at various locations. However, because in most cases a mixture of isolates is present in one particular area, this probably plays a secondary role.

The poor correlation between greenhouse screening and field resistance testing may also be due to several different resistance mechanisms acting under different conditions, as has been observed for Phoma lingam (Koopmann, personal communication). Whereas in the greenhouse the resistance test is performed by a crude inoculation of seedlings, and though infections in the field already occur in autumn, however, V. longisporum exhibits only restricted growth throughout most time of its lifecycle in the plant and it is not until late developmental stages (GS 89) that the fungus can be detected by ELISA. Thus, different defence mechanisms might possibly take effect under natural conditions. However, in both 
the greenhouse and the field, defence mechanisms appear to become operative only after the pathogen has infected the plant and colonized the xylem, demonstrated by the fact that the fungus is placed directly into the vascular system during the greenhouse test and that the pathogen spreads slower and less extensively in less susceptible plants in the field (Figure 2.5).

Finally, the lack of homogeneity of the plant material tested may play a role. The reference cultivars were genetically largely homogenous and showed a consistent response to infection with V. longisporum, albeit the cultivars 'Lion', 'Express', and 'Smart' in 2005/06 and 'Express', 'Falcon', and 'Laser' in 2006/07 did not differentiate in the field as distinctly as compared to the greenhouse tests. On the other hand, the degree of homogeneity in the breeding accessions was unknown. Assuming a lower homogeneity in this material might explain the variability in the test results. Despite the poor correlation between the greenhouse and field resistance trials, several genotypes were identified with equally high levels of resistance in both test systems (SEM 1, SEM 4, DSV 4-06, KWS 28, KWS 39 in 2005/06; WVB 3, SRG 4, Car 321.04, DSV 4 in 2006/07). Particularly with SEM 1 and WVB 3 two genotypes were identified showing an extremely high resistance in the greenhouse screening and in all field trials.

According to Debode et al. (2005), differences in susceptibility of selected cauliflower cultivars (B. oleracea var. botrytis) were related to differences in the presence of $V$. longisporum in the plant. On the basis of re-isolation studies, it was concluded that in cultivars with higher resistance the ascent and proliferation of the fungus was somehow limited. The phenomenon of restricted fungal growth in less susceptible plants is known from other vascular pathogens such as V. dahliae in cotton (Garber \& Houston, 1966; Garas et al., 1986; Wilhelm et al., 1970, 1974) or Fusarium oxysporum in different host plants (Beckman, 1987). Consequently, resistance seems to depend on the rate and extent of defence responses within the vascular system of the host (Beckman, 1990). Further studies on the mechanisms which might operate to restrict the fungal spread in Brassica are presented in Chapter 4.

Summing up these considerations, the results of this study reveal that although the root-dip inoculation method is an efficient and fast tool to characterize the response of Brassica genotypes to $V$. longisporum under greenhouse conditions, this assay has not proven to be sufficient as an isolated screening test method to verify responses under field conditions. Thus, in order to identify resistant genotypes under field conditions, a combination of greenhouse screening and ensuing resistance tests in the field on several locations is 
indispensable. The combination of these two methods, however, appears to be a powerful and reliable approach in the practical selection of resistant breeding lines. 


\section{Chapter 3: Differential interactions of Verticillium longisporum and $V$. dahliae with Brassica napus detected with molecular and histological techniques*}

*) Parts of this chapter have been published in: C. Eynck ${ }^{1}$, B. Koopmann ${ }^{1}$, G. GrunewaldtStoecker $^{3}$, P. Karlovsky ${ }^{2}$, A. v. Tiedemann ${ }^{1}$ (2007) Differential interactions of Verticillium longisporum and V. dahliae with Brassica napus detected with molecular and histological techniques. European Journal of Plant Pathology 118, 259-274

\footnotetext{
${ }^{1}$ Plant Pathology and Plant Protection Division, Department of Crop Sciences, Faculty of Agriculture, Georg-August University Göttingen, Grisebachstraße 6, D-37077 Göttingen, Germany

${ }^{2}$ Molecular Phytopathology and Mycotoxin Research Division, Department of Crop Sciences, Faculty of Agriculture, Georg-August University Göttingen, Grisebachstraße 6, D-37077 Göttingen, Germany ${ }^{3}$ Institute for Plant Diseases and Plant Protection, University of Hannover, Herrenhäuser Strasse 2, D30419 Hannover, Germany
}

\section{Introduction}

Verticillium wilt on oilseed rape (Brassica napus L. spp. oleifera) is caused by the hostadapted, near-diploid fungus Verticillium longisporum (Karapapa et al., 1997b; Zeise \& von Tiedemann, 2001, 2002). The growing area of rapeseed cultivation and the relatively intense crop rotation has rendered this disease an increasing threat to oilseed rape (OSR) production particularly in Europe (Krüger, 1989; Zielenski \& Sadowski, 1995). In Sweden, Verticillium wilt is known to cause economic losses of OSR since the 1960s (Svenson, \& Lerenius, 1987) and has become a significant problem since the 1970s (Dixelius et al., 2005). In the main cropping areas for winter OSR in Germany, the occurrence of $V$. longisporum has increased rapidly since the mid 1980s (Daebeler et al., 1988; Zeise \& Seidel, 1990; Günzelmann \& Paul, 1990). For both winter and spring type OSR, breeding for resistance has been severely hampered by the absence of sufficient resistance in commercially available breeding material, however, recently, some promising genotypes of cabbage (B. oleracea) with enhanced resistance were identified (Happstadius et al., 2003; Dixelius et al., 2005).

A closely related species to $V$. longisporum is $V$. dahliae. This ubiquitous soilborne fungus causes wilt diseases on many economically important crops, including cotton, cucurbits, alfalfa, sunflower, eggplant, mint, strawberry, tomato and potato (Domsch et al., 1980; Schnathorst, 1981; Pegg, 1984; Subbarao et al., 1995; Bhat \& Subbarao, 1999). A 
differentiation of long-spored Verticillium isolates collected from horseradish, classified as Verticillium dahliae var. longisporum, was first made in the early 1960s (Stark, 1961), until the detailed description of distinct morphological, physiological and molecular traits led to the proposition of treating V. longisporum as a distinct species (Karapapa et al., 1997b). Nonetheless, there is still controversy concerning the taxonomy of $V$. longisporum as a separate host-specific species of Verticillium (Fahleson et al., 2004). Thus it has been reported that Brassica crops can occasionally host short-spored Verticillium isolates (Collins et al., 2003) and that $V$. longisporum is able to infect plant species outside the Brassicaceae family (Fahleson et al., 2003; Johansson et al., 2005). Additional confusion has been caused by some misidentification of the two species. In several studies, V. dahliae has been regarded to be the causal agent of Verticillium wilt in Brassica crops (Xiao \& Subbarao, 2000; Söchting \& Verreet, 2004) or on horseradish (Babadoost et al., 2004), without considering that longspored isolates may have been involved.

The host range, epidemiology and infection process on various hosts of $V$. dahliae have been intensely investigated in previous works (Schnathorst, 1981; Beckmann, 1987; Gold et al., 1996; Rowe \& Powelson, 2002). After germination of microsclerotia which is inducible by the root exudates of host plants (Mol \& Scholte, 1995), the fungal hyphae reach the root surface, penetrate the epidermal cells, transverse the root cortex and enter the immature xylem elements (Beckman, 1987). Most of the time of its life cycle $V$. dahliae is constricted to the vascular system, which is a nutrient-limited environment containing low amounts of sugars, inorganic salts, and amino acids, to which the fungus is well adapted (Wood, 1961; Dimond, 1970; Dixon \& Pegg, 1972; Green, 1981; Pegg, 1981, 1985; Van Alfen, 1989). In this liquid environment $V$. dahliae exhibits both hyphal and a "yeast-like" growth, producing conidia budding directly from hyphae, or formed on short phialides (Buckley et al., 1969). With the transpiration stream the released conidia are carried upwards in the vascular elements where they are eventually trapped in bordered pits or at vessel end walls. Colonization proceeds by formation of germ tubes which penetrate into the upstream vessel elements where new conidia are produced (Beckman, 1987; Gold et al., 1996). In the later stages of the disease cycle, as the foliage begins to senesce, the pathogen enters a limited saprophytic growth phase in which microsclerotia are formed in the dying stem parenchyma, thus having a critical role as the primary source of disease in the field (Schnathorst, 1981; Neumann \& Dobinson, 2003).

Currently, little is known about the infection patterns of both $V$. longisporum and $V$. dahliae on roots of B. napus. In order to visualize their colonization of the oilseed rape rhizosphere by confocal laser scanning microscopy (CLSM), we used the green fluorescent protein (GFP) 
from the jellyfish Aequorea victoria (Chalfie \& Kain, 1998; Tsien, 1998) to label both $V$. longisporum and $V$. dahliae. Since the first report on the use of GFP as a vital marker in plantpathogen-interaction studies (Spellig et al., 1996), the GFP transformation has become a common tool in the analysis of various fungal/fungus-like plant pathogens (Lorang et al., 2001). As a particular advantage of the method, plant infection and colonization of GFPexpressing fungi can be followed by fluorescence microscopy in intact plant tissues or tissue sections without requiring cofactors or substrates (Horowitz et al., 2002; Shan \& Godwin, 2004; Bolwerk et al., 2005).

In the present study the GFP-labelling was compared with a technique combining conventional fluorescence staining with confocal microscopy. We describe (i) the Agrobacterium tumefaciens mediated transformation of both $V$. longisporum and $V$. dahliae resulting in stable expression of GFP, (ii) compare the suitability of the GFP reporter gene technique with classical fluorescence staining and (iii) describe new aspects in the early and advanced stages of $V$. longisporum and $V$. dahliae on the roots and in the vascular system of oilseed rape. The microscopical results are further supported by the documentation of disease development by real- time PCR analysis of fungal DNA.

\section{Materials and Methods}

Plant material

The susceptible winter oilseed rape variety 'Falcon', supplied by Norddeutsche Pflanzenzucht Hans-Georg Lembke KG (NPZ), was used in this study. Seeds were double surface-sterilized by sequential immersion in $70 \%$ ethanol for $2 \mathrm{~min}$ and $1 \%$ sodium hypochlorite containing $0.1 \%$ Tween-20 for 15 min under constant shaking (Zou et al., 2004). Subsequently, seeds were washed two times with autoclaved tap water.

\section{Fungal isolates}

Verticillium longisporum isolate VL 43 from Brassica napus and Verticillium dahliae isolate VD 73 (vegetative compatibility group 2B) from Linum usitatissimum were used throughout this work. Both isolates originated from hosts grown in the North of Germany (Zeise \& von Tiedemann, 2001; Zeise \& von Tiedemann, 2002 a; Zeise \& von Tiedemann, 2002 b). Longterm storage was performed as conidial suspensions in a concentration of $1-3 \times 10^{6}$ conidia $\mathrm{mL}^{-1}$ in Czapek Dox medium supplemented with $25 \%$ glycerol at $-80^{\circ} \mathrm{C}$. For propagation, droplets of these suspensions were plated onto potato dextrose agar (PDA) and incubated for 14 days at $23^{\circ} \mathrm{C}$ in the dark. Spores were obtained by gently flooding the dishes with $0.9 \%$ 
$\mathrm{NaCl}$ solution (modified according to Melouk, 1992). Inoculum for root dip inoculation was produced by adding $500 \mu \mathrm{L}$ of the spore stock solution to $250 \mathrm{~mL}$ potato dextrose broth (PDB). The cultures were subsequently incubated for 7 days at $23^{\circ} \mathrm{C}$ on the rotary shaker. The resulting suspension was filtered through sterile gauze. Spore concentration was determined with a haemocytometer and diluted to $1 \times 10^{6}$ spores $\mathrm{mL}^{-1}$.

\section{Bacterial vector strain}

Escherichia coli strain DH5 $\alpha$ (Hanahan, 1983) was used during construction and maintenance of plasmids. Agrobacterium tumefaciens strain AGL-1 (Lazo, Stein \& Ludwig, 1991) was kindly provided by Dr. Susanne Frick, Leibniz Institute of Plant Biochemistry, Halle/Saale. This strain carries the hypervirulent Ti helper plasmid pTiBo542 $\Delta \mathrm{T}$ which lacks one border sequence and thus an intact T-DNA region (Hood et al., 1986; Komari, Halperin \& Nester, 1986; Lazo et al., 1991). For the transformation of plants or fungi, this strain has to carry a binary vector in which the T-DNA is defined by both left and right border sequences (Bevan, 1984). Short-term storage of the Agrobacterium cells was performed on solid LB (Luria Bertani) medium (Maniatis, Fritsch \& Sambrook, 1982), supplemented with $25 \mu \mathrm{g} \mathrm{mL}$ rifampicin and $50 \mu \mathrm{g} \mathrm{mL} \mathrm{m}^{-1}$ carbenicillin at $4^{\circ} \mathrm{C}$. Cells were stored long term as electrocompetent cultures in the freezer at $-80^{\circ} \mathrm{C}$.

\section{Binary vector construction}

The binary vector used in this study was constructed on the backbone of pPK2 (Covert et al., 2001). A fragment of about $2.9 \mathrm{~kb}$ of gGFP (Maor et al., 1998), containing the sgfp gene driven by the Aspergillus nidulans promoter and terminated by the Aspergillus nidulans trpC terminator (Punt et al., 1987), was isolated by digestion with XbaI and EcoRI. sGFP is a variant of GFP carrying S65T mutation, which was constructed with the goal to improve optical properties of GFP expressed in plants and proved superior to wildtype GFP when expressed in fungal mycelium (Maor et al., 1998).

In two different reactions the plasmid pBluescript-SK ${ }^{-}$(Acc. No. X52330, Short et al. 1988) was digested on the one hand with the restriction enzymes AflIII and XbaI and on the other hand with AfIIII and EcoRI, leading to fragments of $450 \mathrm{bp}$ and $2.5 \mathrm{~kb}$ size, respectively. These fragments were ligated with the XbaI/EcoRI fragment from gGFP in a triparental ligation, resulting in the plasmid pBS::gpd::sgfp. Subsequently, the sGFP expression cassette was removed from pBS::gpd::sgfp again by digesting with XbaI and HindIII, which is located downstream to the EcoRI site. The resulting fragment was inserted into the corresponding 
$\mathrm{XbaI} /$ HindIII sites of pPK2. The final construct, pGV04 (Fig. 3.1), was introduced into Agrobacterium tumefaciens by electroporation (Wen-jun \& Forde, 1989) at $2.5 \mathrm{kV}, 400 \mathrm{ohms}$ and $25 \mu \mathrm{F}$ using a cuvette with a $1 \mathrm{~mm}$ gap between the electrodes.

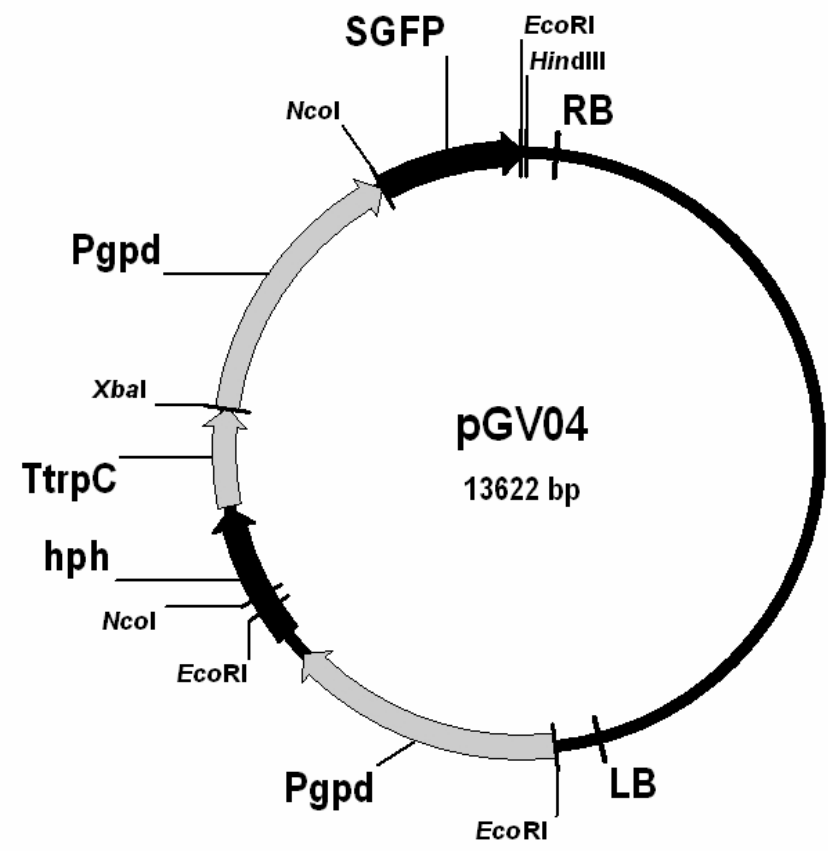

Fig. 3.1. Restriction enzyme map of pGV04, an Agrobacterium binary vector constructed on the backbone of pPK2 (Covert et al., 2001). The T-DNA is running clockwise from the left border (LB) to the right border (RB). Pgpd: Aspergillus nidulans glyceraldehydes 3-phosphate promoter; hph: hygromycin $\mathrm{B}$ resistance as a selection marker; TtrpC: A. nidulans transcriptional terminator.

Agrobacterium- mediated transformation of V. longisporum and V. dahliae

The Agrobacterium tumefaciens strain AGL1, containing the binary vector pGV04, was grown at $28^{\circ} \mathrm{C}$ for $48 \mathrm{~h}$ in $\mathrm{LB}$ medium supplemented with rifampicin $\left(25 \mu \mathrm{g} \mathrm{mL} \mathrm{m}^{-1}\right)$, carbenicillin $\left(25 \mu \mathrm{g} \mathrm{mL}^{-1}\right)$ and kanamycin $\left(50 \mu \mathrm{g} \mathrm{mL}^{-1}\right)$. After reaching an optical density of $\mathrm{OD}_{660}=0.6-0.9$ bacterial cells were harvested and washed with induction medium (IM, Bundock et al., 1995) supplemented with $200 \mu \mathrm{M}$ acetosyringone (AS). Subsequently, the bacterial suspension was diluted with induction medium to $\mathrm{OD}_{660}=0.15$. The cells were grown for an additional period of $6-12 \mathrm{~h}$ before being mixed with an equal volume of a spore suspension of either Verticillium longisporum isolate VL 43 or Verticillium dahliae isolate VD $73\left(1-3 \times 10^{6}\right.$ spores $\left.\mathrm{mL}^{-1}\right)$. From this mixture aliquots of $200 \mu$ l were plated on a cellophane membrane placed on solid cocultivation medium (same composition as IM except that it contains $5 \mathrm{mM}$ glucose instead of $10 \mathrm{mM}$ glucose) supplemented with $200 \mu \mathrm{M}$ 
acetosyringone. After cocultivation at $23^{\circ} \mathrm{C}$ for $60 \mathrm{~h}$ the growing fungal mycelium and the Agrobacteria were suspended in $0.9 \% \mathrm{NaCl}$ solution supplemented with $200 \mu \mathrm{M}$ cefotaxim (for separation from Agrobacterium tumefaciens) and plated on Czapek Dox medium containing hygromycin $\mathrm{B}\left(50 \mu \mathrm{g} \mathrm{mL}^{-1}\right)$ as a selection agent for the transformed fungi and again cefotaxim $(200 \mu \mathrm{M})$. The plates were incubated at $23^{\circ} \mathrm{C}$ and after $8-10$ days discrete colonies developed. Each colony was checked under the fluorescence microscope and those showing the typical GFP fluorescence were subcultured for further studies. To determine their mitotic stability, all transformants were successively cultured in PDB for at least seven generations without supplementation of hygromycin B and checked under the fluorescence microscope. Throughout this subcultivation, the transformants did not show any alterations in growth or colony morphology compared to the wild type.

\section{In vitro root inoculation assay}

Sterilized seeds of Brassica napus cv. 'Falcon' were sown on a cellophane membrane placed on water agar in Petri dishes, preventing the roots from growing into the medium and therefore allowing for an undisturbed microscopic analysis of the interaction between plant roots and fungus. The Petri dishes were sealed with Parafilm and subjected to a light regime of $14 / 10 \mathrm{~h}$ (light/dark) and a temperature of $23 / 20^{\circ} \mathrm{C}$ (day/night) in a climate cabinet. This system kept the intact plants free from contaminations by other micro-organisms. After the plants had developed a well-defined root system, droplets of a spore suspension of either the transformed $V$. longisporum or the transformed $V$. dahliae strain were placed on the cellophane membrane close to the roots.

\section{Gnotobiotic sand inoculation systems}

Sterilized seeds of Brassica napus cv. 'Falcon' were sown in pots $(7 \times 7 \mathrm{~cm})$ containing double-autoclaved silica sand resulting in two plants per pot. This system was used in order to provide conditions for roots approximating the unsterile situation in natural soils, but nevertheless enabling the harvest of complete clean roots without any adhering soil or organic particles. The pots were watered daily and fed two times a week with a full nutrient solution (“Flory Basisdünger", EUFLOR) throughout the experiments. Plants were kept in a controlled environment chamber at $23 / 20^{\circ} \mathrm{C}$ (day/night) and 14-h day length (Philips TL5 HO lamps). Inoculations were either made by direct application of spore suspensions to the sand or by root-dipping. Direct inoculation was conducted one week after germination. The plantlets were inoculated by pouring $8 \mathrm{~mL}$ of a spore suspension of transformed strains of $V$. 
longisporum or $V$. dahliae per plant on the sand surface. After inoculation, all plants were gently watered in order to wash the inoculum into the soil and to evenly spread the spores in the rhizosphere of the plants.

Root-dipping inoculation was used in the experiments for real-time PCR analysis and the documentation of symptom development in the B. napus/V. longisporum and B. napus/V. dahliae interaction. After growing for ten days, seedlings were carefully removed from the sand and the roots washed gently under tap water. Inoculation was performed by dipping the intact roots in a spore suspension of either V. longisporum isolate VL 43 or V. dahliae isolate VD 73 for $30 \mathrm{~min}$. Control plants were dipped in sterilized tap water for the same time. Subsequently, 60 plantlets of each variant (non-inoculated control, VL, VD) were transferred into pots with a sand:soil (1:1) mixture and grown in a climate chamber under standardised environmental conditions (see above).

\section{Assessment of disease development}

Tab. 3.1. Assessment key for scoring disease symptoms induced by Verticillium sp. on young B. napus plants inoculated in the greenhouse (following Zeise, 1992, modified).

\begin{tabular}{|c|c|}
\hline Score & Symptom description \\
\hline 1 & no symptoms \\
\hline 2 & slight symptoms on the oldest leaf (yellowing, black veins) \\
\hline 3 & slight symptoms on the next younger leaves \\
\hline 4 & about $50 \%$ of the leaves show symptoms \\
\hline 5 & more than $50 \%$ of the leaves show symptoms \\
\hline 6 & up to $50 \%$ of the leaves are dead \\
\hline 7 & more than $50 \%$ of the leaves are dead \\
\hline 8 & only apical meristem is still alive \\
\hline 9 & the plant is dead \\
\hline
\end{tabular}

Plants were scored weekly for disease symptoms using an assessment key with nine classes (Table 1; following Zeise, 1992, modified). Scoring was conducted weekly over five weeks. AUDPC values (area under the disease progress curve) were calculated from the disease severity values according to the following formula (Campbell \& Madden, 1990): 


$$
\operatorname{AUDPC}=\sum_{i=1}^{n}\left(y_{i}+y_{i+1} / 2\right) *\left(t_{i+1}-t_{i}\right)
$$

where $y_{i}$ is the disease severity value for observation number $i, t i$ is the corresponding number of days post inoculation of an observation, and $n$ is the number of observations. Statistical analyses were conducted using the computer software StatGraphics. Differences among means were tested using Fisher's least significant difference (LSD).

Staining and microscopy of inoculated plant tissue

All microscopic investigations were conducted with GFP tagged strains. Examinations of the in vitro infection were started $11 \mathrm{~h}$ post inoculation (hpi) and continued at 12-h intervals for the first 4 days using different plants for each time point. Additional observations were made after 3 weeks in order to examine the development of microsclerotia in the root tissue.

Examinations of plants grown in the sand system were started 24 hpi and continued at 24-h intervals for 7 days and at 48 -h intervals in the following week. Furthermore, plants were investigated after 3, 4, and 5 weeks for the extent of colonization of the xylem vessels. For interaction studies with the sGFP tagged strains, whole roots where placed directly on glass slides in drops of water, covered with a cover glass, and examined. For the classical histological studies, whole roots or free hand made sections of roots and stems were stained either with $0.05 \%$ acid fuchsin (C.I. 42685, Merck; $1 \mathrm{~g} 100 \mathrm{ml}^{-1} \mathrm{~A}$. dest.) dissolved 1:10 in lactophenol or in a $1 \%$ acridine orange (C.I. 46005, Merck; 0,1g $100 \mathrm{ml}^{-1}$ A. dest.) solution in water. After rinsing the samples either with lactophenol or water they were mounted on glass slides in drops of lactophenol or water, respectively, closed with a cover slip and immediately examined. Observations were carried out in at least four independent experiments.

Microscopic analyses were performed with a Leica TCS SP2 Confocal Laser Scanning Microscope (CLSM; Leica, Mannheim, Germany). Digital images of GFP tagged strains were acquired by scanning with $488 \mathrm{~nm}$ excitation and 520-540 nm emission filters. Settings for acid fuchsin fluorescence were $543 \mathrm{~nm}$ for excitation and 560-620 nm for emission. Digital images of acridine orange stained specimen were acquired by two-channel-analysis with subsequent drafting of an overlay (488 nm for excitation/ 500-530 nm for emission and 543 $\mathrm{nm}$ for excitation/ $560-650 \mathrm{~nm}$ for emission). Stacks of optical sections were processed to maximum projections. 
DNA extraction and real-time PCR analysis

Ten plants from each treatment were harvested for real-time PCR analysis at weekly intervals from 7 dpi (days post inoculation) until 35 dpi. Hypocotyls and leaves were separated resulting in twenty tissue samples and PCR analyses for each treatment. Roots were not included in the analysis as a proper discrimination between fungal biomass in the roots from fungus merely attached to the root surface through inoculation is not possible.

First, the plant tissue was ground in liquid nitrogen using a mortar and a pestle resulting in a fine powder. DNA extraction was conducted using the DNeasy Plant Mini Kit from Qiagen (Hilden, Germany). Fungal biomass was quantified by determination of fungal DNA in infected plant extracts with real-time PCR. Primers OLG 70 (CAGCGAAACGCGATATGTAG) and OLG 71 (GGCTTGTAGgGgGTTtAGA) (P. Karlovsky, unpublished) served to multiply a fragment specific for both $V$. longisporum and $V$. dahliae. For amplification and melting curve analysis the iCycler System (BioRad, Hercules, CA, USA) was used.

The reaction mixture consisted of $\mathrm{NH}_{4}$-reaction buffer $\left(16 \mathrm{mM}\left(\mathrm{NH}_{4}\right)_{2} \mathrm{SO}_{4}, 67 \mathrm{mM}\right.$ Tris- $\mathrm{HCl}$, $0.01 \%(\mathrm{v} / \mathrm{v})$ Tween-20, $\mathrm{pH} 8.8$ at $25^{\circ} \mathrm{C}$, Bioline, Luckenwalde, Germany), $3 \mathrm{mM} \mathrm{MgCl}_{2}$ (Bioline, Luckenwalde, Germany), $0.2 \mathrm{mM}$ of each dATP, dTTP, dCTP and dGTP (Bioline, Luckenwalde, Germany), $0.3 \mu \mathrm{M}$ of each primer, $0.25 \mathrm{u}$ BIOTaq DNA polymerase (Bioline, Luckenwalde, Germany), 10 nM fluorescein (BioRad, Hercules, CA, USA), 100,000 times diluted SYBR Green I solution (Invitrogen, Karlsruhe, Germany), $2 \mu 1$ of template DNA and $\mathrm{ddH}_{2} \mathrm{O}$ up to $25 \mu \mathrm{l}$. The quantification of PCR products was performed using filters with optimal settings for SYBR Green I which are $490 \pm 10 \mathrm{~nm}$ for excitation and $530 \pm 15 \mathrm{~nm}$ for emission.

PCR amplification was carried out using an initial denaturation step for $2 \mathrm{~min}$ at $94^{\circ} \mathrm{C}$, which is followed by 36 reaction cycles consisting of a $20 \mathrm{~s}$ denaturation step at $94^{\circ} \mathrm{C}$, an annealing step for $30 \mathrm{~s}$ at $59^{\circ} \mathrm{C}$ and $40 \mathrm{~s}$ at $72^{\circ} \mathrm{C}$. The final elongation was performed for $5 \mathrm{~min}$ at $72^{\circ} \mathrm{C}$. During the amplification process, the detection of fluorescence was carried out in the annealing step of each cycle. To verify amplification of the specific target DNA, a melting curve analysis was included. Melting curves were acquired by heating the samples to $95^{\circ} \mathrm{C}$ for $1 \mathrm{~min}$, cooling to $55^{\circ} \mathrm{C}$ for another min and then slowly increasing the temperature from $65^{\circ} \mathrm{C}$ to $95^{\circ} \mathrm{C}$ at the rate of $0.5^{\circ} \mathrm{C} \mathrm{s}^{-1}$, with a continuous measurement of the fluorescence. The amount of DNA of $V$. longisporum and $V$. dahliae, respectively, was estimated from a calibration curve using increasing amounts of genomic $V$. longisporum DNA from 0.5 to 64.0 pg. Preliminary studies confirmed that the amplification is not hampered by the plant matrix. 
The concentration of $V$. longisporum DNA used for the construction of the calibration curve was estimated by densitometry of agarose gels stained with ethidium bromide, using Lambda Phage DNA as a standard.

\section{Results}

Disease development

Fourteen days after inoculation, first disease symptoms appeared on V. longisporum infected plants, in form of chlorosis and dark coloured veins especially on older leaves (Table 2). At 21 dpi, all plants inoculated with $V$. longisporum showed stunted growth and moderate to less severe disease symptoms. Thus, $20 \%$ of the plants had symptoms on 50 or more percent of the leaves. This corresponds to disease scores from 4 to 8 (see Table 3.1). In the following weeks the disease proceeded until 35 dpi when nearly half of the inoculated plants showed severe disease symptoms. Plants inoculated with $V$. dahliae did not express any symptoms significantly different from the control. Occasionally, they even looked healthier than the control plants. The corresponding AUDPC values were 46.6 for the control, 45.9 for $V$. dahliae and 71.1 for plants inoculated with V. longisporum.

Tab. 3.2: Means of disease scores on B. napus plants inoculated with $V$. longisporum (+ VL) or $V$. dahliae (+ VD) compared to control plants treated with water. Standard error is shown in brackets. Values followed by the same letter do not differ on a significance level of $\mathrm{P} \leq$ 0.05 (LSD). For disease assessment key see table 1.

\begin{tabular}{l|c|c|c|c|c}
\hline & \multicolumn{5}{|c}{ Time post inoculation (dpi) } \\
Treatment & 7 & 14 & 21 & 28 & 35 \\
\hline control & $1.00( \pm 0.00) \mathbf{a}$ & $1.00( \pm 0.00) \mathbf{a}$ & $2.00( \pm 0.00) \mathbf{a}$ & $2.40( \pm 0.15) \mathbf{a}$ & $2.50( \pm 0.17) \mathbf{a}$ \\
$+\mathrm{VD}$ & $1.00( \pm 0.00) \mathbf{a}$ & $1.00( \pm 0.00) \mathbf{a}$ & $2.00( \pm 0.00) \mathbf{a}$ & $2.31( \pm 0.15) \mathbf{a}$ & $2.50( \pm 0.16) \mathbf{a}$ \\
$+\mathrm{VL}$ & $1.00( \pm 0,00) \mathbf{a}$ & $1.42( \pm 0.23) \mathbf{b}$ & $3.31( \pm 0.27) \mathbf{b}$ & $3.35( \pm 0.31) \mathbf{b}$ & $4.58( \pm 0.79) \mathbf{b}$ \\
\hline
\end{tabular}

GFP expression in $V$. longisporum and $V$. dahliae

Overall, 33 transgenic V. longisporum and $20 \mathrm{~V}$. dahliae isolates were obtained. Eighty percent of the hygromycin B-resistant $V$. dahliae and $60 \%$ of the obtained $V$. longisporum isolates expressed the GFP, which was a success rate similar to reports either on Agrobacterium-mediated transformation (Lagopodi et al., 2002; Oren et al., 2003) or electroporation (Robinson \& Sharon, 1999). GFP expression was generally high and uniform in conidia and hyphae with the exception of vacuoles that did not show any fluorescence and appeared as dark areas in the fungal cytoplasm (Fig. 2, A-D). In contrast to this, 
microsclerotia did not fluoresce at all, probably because of the melanin deposited in the cell walls. GFP expression remained stable after successive transfers on Czapek Dox medium with and without hygromycin B.

Suitability of GFP-tagged strains vs. classical staining methods

In this study, we compared the suitability of tagging fungi with GFP with conventional fluorescence staining for plant-fungus interaction studies. Due to the fact that GFP is constitutively expressed in the cytoplasm of the transformed fungi, only younger hyphae show bright fluorescence and are thus well visible under the fluorescence microscope. This hampers a comprehensive analysis of all stages of colonization and infection at one particular time point. In order to localize the fungal structures in the plant tissue we used the overlay feature of the CLSM in order to generate compound images of normal transmission underneath the corresponding fluorescence image. The result, however, was not satisfactory and allowed only for a rough localization of the fungus in the plant tissue (Fig. 3.2 E, F).

By applying acid fuchsin or acridine orange as conventional dyes we obtained a strong staining of all fungal structures but also a faint but distinct staining of cell walls and the plasma membranes allowing for accurate studies of the pathogen invading the plant tissue. As a further problem with the GFP samples, photobleaching occurred after repeated and extended scanning on the same sample sites. In general, this problem can be overcome by reducing the energy level of the laser; however, this option is limited by the fluorescence intensity of the referring object. Regarding all these facts, we concluded that the conventional staining techniques combined with CLSM were superior to the GFP tagging for in situ studies of pathogens at least in the present pathosystems and we therefore decided to continue further studies with fuchsin and acridinorange.

Plant-pathogen interaction studies

Spores of $V$. longisporum and $V$. dahliae started to germinate after in vitro infection $11 \mathrm{hpi}$. Usually, a secondary germ tube developed in opposite position to the emergence site of the primary germ tube. In the sand culture system, hyphae of both $V$. longisporum and V. dahliae were found 24 hpi intensely interwoven with the root hairs close to the main and lateral roots. A tight attachment of hyphae to the root hairs was observed at random positions along the root hair zone for both fungi (Fig. 3.3, A-C). The root tip was the only part of the root which was not colonised. At $24 \mathrm{hpi}$, V. dahliae already showed colonization of the root to a much lesser extent than $V$. longisporum and started to produce ample masses of conidia in the 
vicinity of root hairs (Fig. 3.3, B, D). In contrast, the formation of conidia by V. longisporum was never observed outside the roots throughout the investigations.

Hyphae of $V$. longisporum showed a directed growth on and along the root hairs towards the root surface. At 36 hpi, the first contacts between hyphae and the root surface were observed. After attachment to the root surface, hyphae of $V$. longisporum grew strictly following the grooves of the junctions of the epidermal cells. At 48 hpi, the root surface was covered with a closely attached hyphal net depicting the cellular structure of the root epidermis (Fig. 3.3, E). In contrast, growth of $V$. dahliae hyphae on the root surface was random not following any such pattern (Fig. 3.3, F).

At $60 \mathrm{hpi}$, first penetrations of $V$. longisporum into the roots were observed. The fungus entered the root tissue by direct penetration of the epidermal cells without forming any conspicuous infection structures like appressoria or hyphopodia. Only slight hyphal swellings were formed by hyphae before entering epidermal cells (Fig. 3.4, A), probably due to the accumulation of cytoplasm in the hyphal tip as a response to the mechanical resistance of the plant tissue. Plant cell walls were perforated by a thin penetration peg. In the lumen of the epidermal cells, the hyphae regained their regular diameter (Fig. 3.4, B). Subsequently, hyphae grew intracellularly and intercellularly in the root cortex, in a more or less directed manner towards the central cylinder (Fig. 3.4, C). Whenever growing through cell walls, hyphae showed the typical sequence of swelling, constriction to a narrow infection peg and regaining the regular size after penetration (Fig. 3.4, D). Remarkably, the roots, although intensely colonised by $V$. longisporum, did not show any responses such as discoloration or necrotic lesions. Even host cells invaded by intracellular hyphae showed an intact structure of the cytoplasm.

$V$. dahliae was also able to penetrate the root tissue, but this occurred much less frequent (Fig. $3.4, \mathrm{E})$. In the root cortex, $V$. dahliae was preferentially found in the intercellular rather than the intracellular space (Fig. 3.4, F). Also, in the in vitro inoculation system $V$. dahliae started to produce microsclerotia already after 2 weeks. Interestingly, microsclerotia of $V$. dahliae were not formed in the roots or on the root surface but were scattered on the surrounding medium. In contrast, microsclerotia of $V$. longisporum were consistently formed in the root cells which were filled with microslerotia 3 weeks after inoculation.

Colonisation of the xylem vessels of the shoot with $V$. longisporum started three weeks after inoculation. However, the fungus never managed to colonise the entire vascular system. Moreover, it was restricted to individual vessels which, however, were entirely filled up with mycelium (Fig. 3.5, A, B). Adjacent xylem vessels were easily invaded through 
plasmodesmata (Fig. 3.5, A). Conidia were formed either by budding (Fig. 3.5, C, D) or on simple conidiophores (not shown).
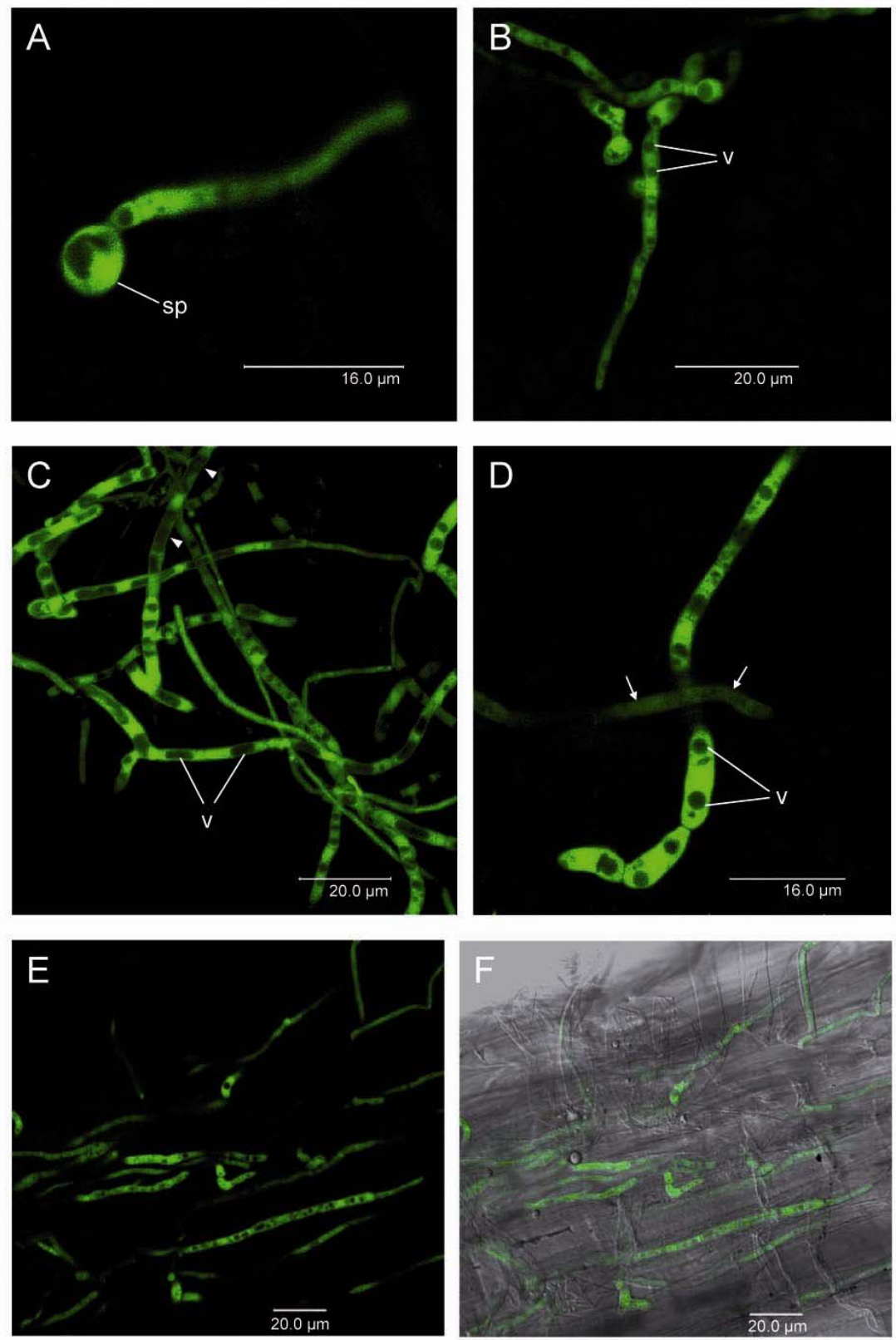

Fig. 3.2. Confocal microscopy image of Verticillium longisporum and V. dahliae expressing GFP. A. Germinating spore of $V$. longisporum. B. Hyphae of $V$. dahliae, vacuoles of fungal cells are visible as dark areas in the fluorescing cytoplasm. C. Mycelium of V. longisporum showing hyphal dimorphism with very thin and normal, vacuolated hyphae. Arrow heads mark areas of weak fluorescence. D. Hyphae of $V$. longisporum. Arrows point at a very weak glowing hypha. $\mathrm{sp}=$ conidiospore, $\mathrm{v}=$ vacuole. E. Fluorescence image of GFP-tagged mycelium of $V$. longisporum on a root of B. napus (48 hpi). F. Compound micrograph of bright field transmission and corresponding fluorescence images (same as $\mathbf{E}$ ). 

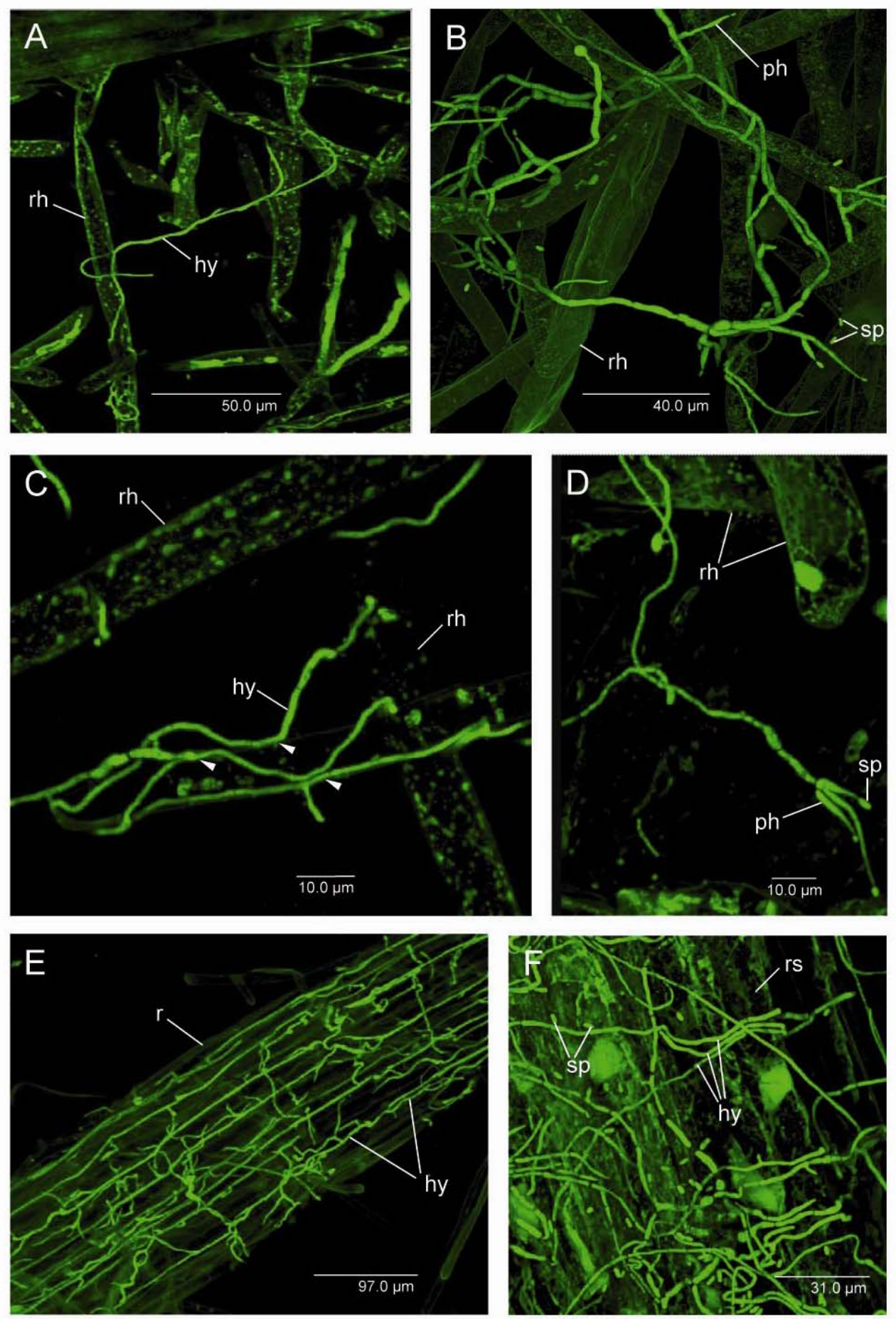

Fig. 3.3. Early stages of root colonization by $V$. longisporum and $V$. dahliae on oilseed rape, as observed by confocal laser scanning microscopy after staining with acid fuchsin. The classical staining method results in a stable and homogenous bright green fluorescence of the entire fungal biomass and a faint background staining of the plant tissue. A-D: 24 hpi; E, F: 48 hpi. A. Contact of hyphae of $V$. longisporum with root hairs. B. Intermingling of hyphae of $V$. dahliae with root hairs. Already at this stage the fungus produces spores. C. Attachment (arrow heads) of fungal hyphae of $V$. longisporum to root hairs. D. V. dahliae, production of conidiospores in between root hairs. E. Hyphae of $V$. longisporum growing along the junctions of the epidermal cells forming a network. F. V. dahliae growing in an undirected manner on the root surface of $B$. napus. hy $=$ hypha, $\mathrm{ph}=$ phialide, $\mathrm{r}=$ root, $\mathrm{rh}=$ root hair, $\mathrm{sp}$ $=$ conidiospore. 

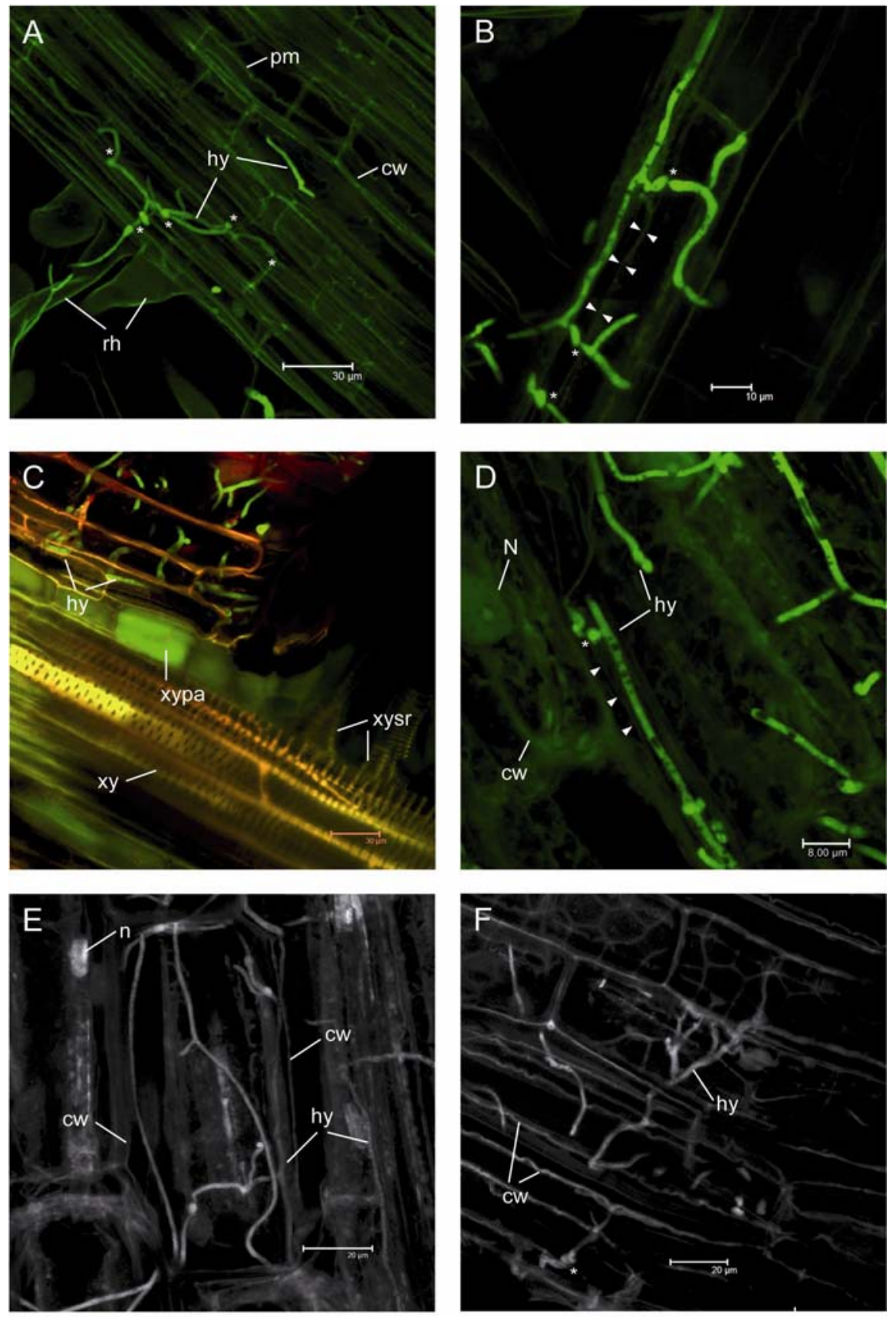

Fig. 3.4. Advanced stages of the interaction of $V$. longisporum and $V$. dahliae with roots of $B$. napus. Confocal laser scanning microscopy analysis after staining with acid fuchsin and acridine orange (C.). A-C, E, $\mathbf{F}=60 \mathrm{hpi} ; \mathbf{D}=96$ hpi. A. Hyphae of $V$. longisporum growing along a root hair towards the root surface, penetrating an epidermal cell and growing further into the root cortex. Asterisks mark the points of intracellular penetration through plant cell walls. B. Intracellular growth of $V$. longisporum in the root cortex. Arrow heads mark the plant cell wall which is penetrated two times (asterisks). After penetration, hyphae regain their regular diameter. C. Directed growth of $V$. longisporum in the root cortex towards the xylem. Arrow heads assign points of penetration. D. Magnified view on the penetration of a plant cell wall (arrow heads) by $V$. longisporum in the root cortex. Asterisk marks the swelling of the hypha before penetrating with a thin penetration peg. E. Growth of $V$. dahliae in the root cortex of $B$. napus. Asterisk marks a primary penetration event. F. Mainly intercellular growth of $V$. dahliae in the root cortex. $\mathrm{cw}=$ cell wall, hy = hypha, $\mathrm{n}=$ nucleus, $\mathrm{rh}=$ root hair, $\mathrm{xy}=$ xylem, $x y p=$ xylem parenchyma, $x y s r=$ xylem of secondary root. 

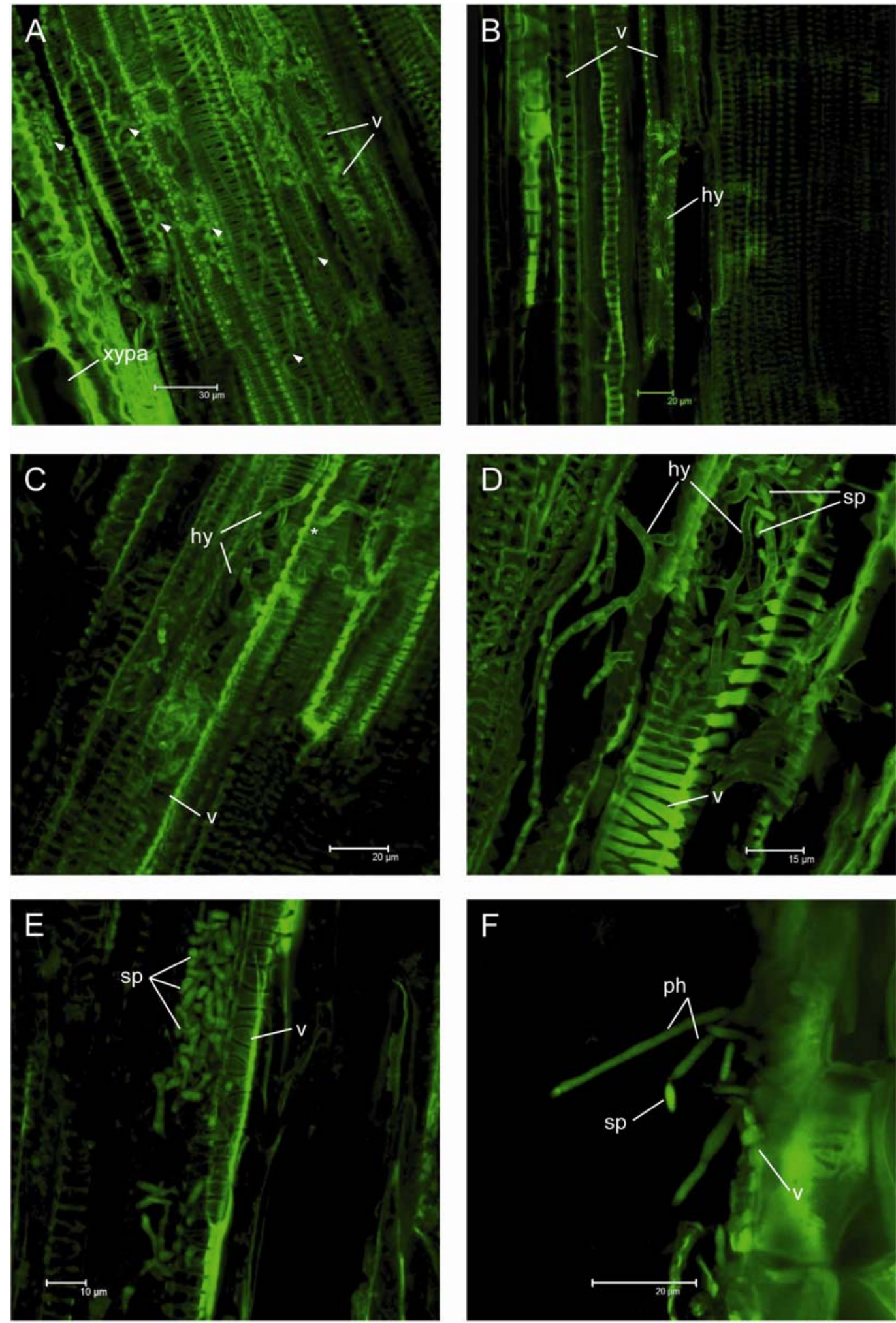

Fig. 3.5. Colonization of the xylem of $B$. napus by $V$. longisporum. Confocal laser scanning microscopy analysis after staining with acid fuchsin $(21 \mathrm{dpi})$. A. Hyphae of $V$. longisporum in xylem vessels of $B$. napus (arrow heads). B. Colonization of a single vessel element which is filled with mycelium. C. Hyphae of $V$. longisporum in xylem vessels of $B$. napus. Proliferation of mycelium into adjacent vessels through plasmodesmata (asterisk). D. Hyphal growth and production of conidiospores in a xylem element. E. Conidiospores clumped together at the end of a tracheid. F. Conidia produced by simple conidiophores in a vessel element. Hy = hypha, $\mathrm{ph}=$ phialides, $\mathrm{sp}=$ conidiospores, $\mathrm{v}=$ vessel elements. 
Real time-PCR analysis
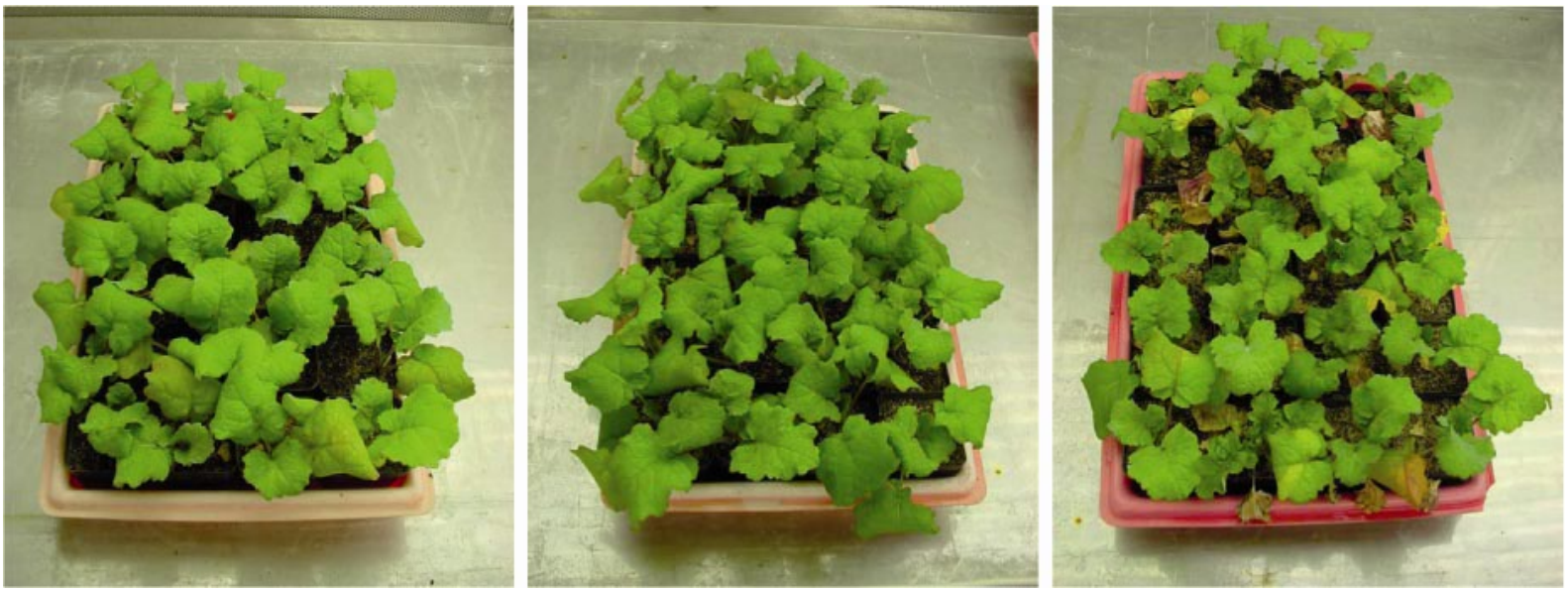

Fig. 3.6. Winter oilseed rape cultivar 'Falcon' inoculated with $V$. dahliae (center) and $V$. longisporum (right) compared to mock-inoculated control (left) (21 dpi).

Seven days post inoculation both $V$. longisporum and $V$. dahliae were detectable in the hypocotyls of infected plants (Table 3.3). The amount of $V$. longisporum DNA was substantially higher than that of $V$. dahliae, corroborating the histological studies. The amount of $V$. longisporum continued to increase in the hypocotyl until $35 \mathrm{dpi}$, whereas $V$. dahliae remained on a very low level. In leaves, $V$. longisporum was first detectable in one single plant at $14 \mathrm{dpi}$, then slightly increased until $28 \mathrm{dpi}$, and sharply spread at $35 \mathrm{dpi}$. In contrast, no significant colonisation of $B$. napus leaves with $V$. dahliae was detected throughout the time of observation.

Tab. 3.3: Detection of Verticillium-DNA with real-time PCR in tissue samples (means of the amount of fungal DNA in leaves and hypocotyls; \pm standard error) of B. napus seedlings inoculated with $V$. longisporum or $V$. dahliae.

\begin{tabular}{|c|c|c|c|c|}
\hline \multirow{2}{*}{ Tissue } & \multicolumn{2}{|c|}{ leaves } & \multicolumn{2}{|c|}{ hypocotyl } \\
\hline & ng VL-DNA/g FW & ng VD-DNA/g FW & ng VL-DNA/g FW & ng VD-DNA/g FW \\
\hline 7 & $0.00 \quad( \pm 0.00)$ & $0.00 \quad( \pm 0.00)$ & $2.88 \quad( \pm 0.89)$ & $0.53 \quad( \pm 0.18)$ \\
\hline 14 & $0.01 \quad( \pm 0.01)$ & $0.00 \quad( \pm 0.00)$ & $9.94 \quad( \pm 2.79)$ & $0.18 \quad( \pm 0.09)$ \\
\hline 21 & $0.51 \quad( \pm 0.29)$ & $0.02 \quad( \pm 0.02)$ & $3.95 \quad( \pm 1.65)$ & $0.05 \quad( \pm 0.02)$ \\
\hline 28 & $0.59 \quad( \pm 0.26)$ & $0.01 \quad( \pm 0.01)$ & $10.41 \quad( \pm 1.52)$ & $2.62 \quad( \pm 1.18)$ \\
\hline 35 & $6.89 \quad( \pm 5.37)$ & $0.06( \pm 0.06)$ & $25.58 \quad( \pm 6.79)$ & $0.24( \pm 0.12)$ \\
\hline
\end{tabular}




\section{Discussion}

This study reports on the Agrobacterium tumefaciens mediated transformation of the phytopathogenic fungi $V$. longisporum and $V$. dahliae, resulting in the stable expression of GFP. This transformation method proved to be an effective tool to introduce foreign genes into the genome of Verticillium species, as indicated by the relatively high number of gained transformants. In comparison, attempts of a DNA-mediated transformation of $V$. dahliae spheroplasts resulted in a low transformation efficiency (Dobinson, 1994). The transformants obtained in this study were indistinguishable from the wild type strain concerning colony morphology, growth rate and pathogenicity/aggressiveness (data not shown). However, the transformants showed differences in the fluorescence intensity due to different levels of GFP expression. This may be caused by positional effects resulting from different sites of integration into the fungal genome. The phenomenon of older hyphae showing a reduced or no expression of GFP has been earlier described in studies on Leptosphaeria spp. and Oculimacula spp (Eckert et al., 2005). This may be due to the fact that the cytoplasm in senescing mycelium is translocated from the older into younger hyphae.

In our studies we directly compared GFP fluorescence with conventional staining with a fluorescence dye. This analysis clearly revealed a superior performance of the applied fluorochromes as the entire mycelium was made visible regardless of its physiologic state. In addition, the faint unspecific staining of the plant tissue enabled a proper localization of the fungus in the host. Nevertheless, there are still several advantages in the GFP labelling of plant pathogens. First, GFP fluorescence requires no cofactors or substrates and allows for a fast processing of the plant material. Further, the risk of potential artefacts is negligible due to the absence of additional chemicals. This enables time-lapse observations in vivo (Lagopodi et al., 2001). Also, studies of pathogens in non-sterile conditions would make GFP tagging the preferential approach.

There are several similarities from our microscopic studies of $V$. longisporum on and in $B$. napus roots with earlier reports on the infection process of $V$. dahliae on a wide range of host plants (Schnathorst, 1981; Beckmann, 1987; Gold et al., 1996). However, our studies also provide significant novel information about colonization and infection. This particularly applies to the early interaction, including recognition and first contacts between host and pathogen. We observed that $V$. longisporum initially approaches the roots by following the root hairs. This may be due to a stronger chemical attraction of the fungus to the root hairs than the root surface, as suggested in an earlier study with Fusarium oxysporum f. sp. radicislycopersici on tomato roots (Lagopodi et al., 2001). Thereafter, hyphae of V. longisporum 
attach to the root surface where they preferably spread in a typical pattern of superficial hyphae. Primary infection occurs either at the junctions of epidermal root cells or directly into epidermal cells. We never observed penetration at the very root tip or into root hairs. This is in contrast to a former study (Zhou et al., 2006) where infection by V. longisporum was primarily localized in lateral roots or root hairs. Further, there was no indication that natural wounds from emerging lateral roots are necessary for infection, which is in agreement with previous studies on GFP-expressing Fusarium oxysporum f. sp. radicis-lycopersici on tomato roots (Lagopodi et al., 2001) and Fusarium verticillioides on maize roots (Oren et al., 2002). It may therefore be speculated whether there exists a common mode of root colonization and infection by vascular pathogens, in which the pathogens are chemically directed to the root hair zone where they first attach and then penetrate directly into the epidermal cells (Oren et al., 2002).

The observation that even massive inoculation with $V$. longisporum results in a colonisation merely restricted to individual xylem vessels, while others remain entirely free of the fungus, has not been reported so far. Nonetheless, $V$. longisporum was able to penetrate into adjacent vessels after being trapped at vessel end walls, which was similar to recent studies of $V$. longisporum (Zhou et al., 2006) and V. albo-atrum (Heinz et al., 1998), but has not been shown for $V$. dahliae so far. This partial colonisation may be an explanation for the absence of wilting symptoms in $V$. longisporum infected oilseed rape, as observed both in the greenhouse and in the field. However, one has to keep in mind that wilting is not only caused by the physical blocking of vessels but may also be induced by wilting toxins, which however are yet unknown for $V$. longisporum.

The interaction of $V$. dahliae with $B$. napus roots completely differs from $V$. longisporum. The undirected growth of hyphae which are only loosely attached to the root surface and the early, massive production of conidia and microsclerotia outside the root tissue strongly suggest that B. napus is not a suitable host plant for $V$. dahliae. This colonization pattern might be the result of a stress or deficiency situation derived from a non-host interaction. Nevertheless, $V$. dahliae was infrequently able to penetrate and colonize the root tissue, however, it rarely reached the shoots or leaves as indicated by histological and real-time PCR analyses. In conclusion, the present results demonstrate that the poor susceptibility of $B$. napus to $V$. dahliae is only partly due to the restriction of penetration but mainly related to inhibition of systemic growth into the shoot, which is in agreement with earlier observations (Zhou et al., 2006). While $V$. longisporum, upon penetration, readily spreads into the vascular system, the systemic growth of $V$. dahliae is strongly inhibited due to yet unknown factors. This kind of 
restricted invasion of a vascular pathogen represents an interesting intermediate case of parasitism, as root penetration is not followed by invasive spread in the host. As disease symptoms are lacking, the lifestyle of $V$. dahliae after infection resembles an endophytic state and implies a systemic non-host resistance. Similarly, a restriction to the basal plant parts has been found with certain other wilt pathogens in plants with enhanced host plant resistance (Beckman, 1987).

The mechanisms governing the expression of this specific type of non-host resistance are not known. Increased synthesis of the aromatic glucosinolate gluconasturtiin in the roots appeared to be a major factor in the active resistance response of oilseed rape against a non-pathogenic $V$. dahliae strain which failed to colonize the plant (Karapapa et al., 1997a). The pathogenic $V$. longisporum strain was either capable to suppress gluconasturtiin synthesis in roots, or failed to trigger its synthesis by avoiding recognition. This implies an adaptation of $V$. longisporum to oilseed rape as host, potentially by being less sensitive to this kind of hostspecific defence, as compared to $V$. dahliae. This is corroborated by studies in which the pathogenicity of $V$. longisporum on high and low glucosinolate producing genotypes of $B$. napus did not differ (Heale \& Karapapa, 1999). Similarly, Zhou et al. (2006) did not find any differences in the growth of $V$. longisporum on agar containing plant extracts from high or low glucosinolate oilseed rape varieties.

Finally, the present study provides cytological evidence that infrequent infection of oilseed rape with $V$. dahliae may occur, but a vascular colonization and disease development can be excluded. This finding confirms earlier studies on host range (Zeise \& von Tiedemann, 2002) and is of importance in fields where oilseed rape is grown in rotation with susceptible hosts of V. dahliae like potato, strawberry or sugar beet (Pegg \& Brady, 2002), and where enhanced soil infestation with microsclerotia from both Verticillium species may occur. 


\section{Chapter 4: Mechanisms involved in resistance of Brassica spp. to $V$. longisporum*}

*) Parts of this chapter have been submitted for publication in: Eynck, C. ${ }^{1}$, Koopmann, B. ${ }^{1}$, Karlovsky, P. ${ }^{2}$, von Tiedemann, A. ${ }^{1}$ : Internal resistance in winter oilseed rape (Brassica napus L. oleifera) inhibits systemic spread of the vascular pathogen Verticillium longisporum.

${ }^{1}$ Plant Pathology and Plant Protection Division, Department of Crop Sciences, Faculty of Agriculture, Georg-August University Göttingen, Grisebachstraße 6, D-37077 Göttingen, Germany

${ }^{2}$ Molecular Phytopathology and Mycotoxin Research Division, Department of Crop Sciences, Faculty of Agriculture, Georg-August University Göttingen, Grisebachstraße 6, D-37077 Göttingen, Germany

\section{Introduction}

Verticillium longisporum (ex. V. dahliae var. longisporum Stark; comb. nov. Karapapa) is a soilborne fungal pathogen causing vascular diseases of cruciferous plants (Karapapa et al., 1997b; Zeise \& von Tiedemann, 2001, 2002). Verticillium wilt is a novel disease on oilseed rape (Brassica napus L. spp. oleifera), threatening its production particularly in the northern European countries (Krüger, 1989; Zielenski \& Sadowski, 1995; Dunker et al., 2007).

Like the closely related species $V$. dahliae, $V$. longisporum accumulates durable microsclerotia in the soil from which plant roots are attacked (Schnathorst, 1981; Beckmann, 1987; Gold et al., 1996). After germination of microsclerotia, which is triggered by root exudates from the host plant (Schreiber \& Green, 1963; Huisman, 1982; Olsson \& NordbringHertz, 1985; Mol et al., 1995; Gödecke, 2007, unpublished data) the fungal hyphae grow towards the root surface and penetrate the root epidermal cells near the root tips (Zhou et al. 2006; Eynck et al., 2007). Then, the fungus traverses the root cortex inter- and intracellularly and enters into the xylem. During most of its life cycle $V$. longisporum is confined to the vascular system, a nutrient-limited environment to which the fungus is well adapted (Wood, 1961; Dimond, 1970; Dixon \& Pegg, 1972; Green, 1981; Pegg, 1981, 1985; Van Alfen, 1989). The pathogen spreads with growing hyphae and/or segregated conidiospores floating with the transpiration stream into upper parts of the plant vascular system. As the host tissue turns to senescence, the pathogen enters a final saprophytic growth stage in which microsclerotia are abundantly formed in the dying stem parenchyma.

The fact that Verticillium in oilseed rape cannot be controlled with fungicides and the extended survival of microsclerotia in the soil (Heale \& Karapapa, 1999) limits the control of 
the disease on either cultural practices such as wider crop rotation or the use of resistant cultivars. Until recently, breeding for resistance has been hampered by the lack of sufficient resistance in commercially available breeding material. However, a promising level of resistance to $V$. longisporum has been identified in cabbage (B. oleracea; Happstadius et al., 2003; Dixelius et al., 2005). More recently, significant improvement in resistance of B. napus to $V$. longisporum has been achieved by hybridization of resistant progenitor species of $B$. oleracea with B. rapa (Rygulla et al., 2007a, b).

Resistance to wilt pathogens such as Verticillium is supposed to depend predominantly on the physical restriction and chemical inhibition of the pathogen during the systemic phase of colonization (Nicholson \& Hammerschmidt, 1992). Unlike in many biotrophic and some necrotrophic interactions, there is no complete resistance to vascular infection on the host plant resistance level (Beckman, 1987). Thus host plants may lack severe symptoms although being systemically colonized which is commonly denoted as 'resistant' instead of 'tolerant' (Garber \& Houston, 1966; Bishop \& Cooper, 1984; Pegg \& Brady, 2002). This specific type of 'internal' resistance is usually based on the rapid build-up of mechanical barriers, vascular occluding gels or tyloses preventing or delaying the linear spread of the pathogen in the vascular system (Talboys, 1958; Sinha \& Wood, 1968; Elgersma et al., 1972; Tjamos \& Smith, 1974, 1975; Beckman \& Talboys, 1981; Beckman, 1987; Beckman, 1990).

Phenolic compounds from the plant phenylpropanoid pathway play an important role in defence to pathogen infection either as preformed or post-infectional defence factors (Mansfield, 1983; Goodman et al., 1986; Candela et al., 1995). Thus, they have been assigned to various important biological functions in defence such as cell wall reinforcement and antimicrobial activity (Bell, 1970; Baranowski et al., 1980; Shuen \& Buswell, 1992; Snook et al., 1992; Tuncel \& Nergiz, 1993), as modulators of plant hormones or in defence signalling as well as scavengers of reactive oxygen species (Nicholson \& Hammerschmidt, 1992; Dixon \& Paiva, 1995; Dixon et al., 1996; Ryals et al., 1996; Wink, 1997; Weisshaar \& Jenkins, 1998; Dixon \& Steele, 1999; Beckman, 2000).

Lignin and cell wall lignification (Lewis \& Yamamoto, 19990; Bernards \& Lewis, 1992; Whetten \& Sederoff, 1995) are important principal structural components of secondary vascular tissues and fibres in higher plants (Humphreys \& Chapple, 2002; Dixon et al., 1996) and may therefore play a crucial role in the defence of vascular pathogens. Lignin plays a fundamental role in mechanical support, solute conductance and disease resistance (Barber \& Mitchell, 1997; Harakava, 2005). Deposition of lignin, lignin-like polymers and other wallbound phenolic materials may be responses to mechanical damage or wounding or to 
microbial infection (Boudet et al., 1995). In addition to cell wall strengthening and increased cell wall rigidity, lignin deposition is supposed to decrease the diffusion of toxins released from pathogenic fungal hyphae, and to reduce the transfer of nutrients from the host to the pathogen (Vance et al., 1980; Nicholson \& Hammerschmidt, 1992).

Resistance to Verticillium has been found in several cruciferous host plants, like cauliflower (Koike \& Subbarao, 1994; Debode et al., 2005), Japanese cabbage (Kemmochi et al., 2000), horseradish (Atibalentja \& Eatburn, 1998), cabbage and turnip rape (Happstadius et al., 2003; Dixelius et al., 2005; Rygulla et al., 2007a, b) as well as in oilseed rape (Steventon, 2002b). Notably the Brassica oleracea gene pool, i.e. the Brassica $\mathrm{C}$ genome contains resistance traits to V. longisporum (e.g. Happstadius et al., 2003). Oilseed rape (genome AACC) is an amphihaploid species derived from interspecific hybridizations between turnip rape (B. rapa L. syn. campestris; AA) and cabbage (B. oleracea, genome CC). Hence, resistance in B. napus might rely on similar mechanisms as in the progenitor species B. oleracea. However, until now, mechanisms underlying plant defence responses of $B$. napus to $V$. longisporum have not been studied and a possible coherence between the modes of resistance in the closely related Brassica species has not been investigated so far.

This study reports on histochemical and physiological data elucidating the mechanisms involved in the genotype-specific resistance of B. napus to V. longisporum. In particular, resistance is related to changes in the phenolic composition of root and stem tissues, to alterations in the vascular cell walls and to vessel occlusions occurring in the vascular tissue during various stages of infection and systemic colonization. In parallel, the pathogen spread in a susceptible and a resistant B. napus cultivar is followed by qPCR. This study provides the first histological and biochemical characterization of quantitative resistance in rapeseed cultivars against $V$. longisporum. 


\section{Material and Methods}

Plant material

A susceptible variety of winter oilseed rape, 'Falcon', provided by Norddeutsche Pflanzenzucht Hans-Georg Lembke KG (NPZ, Hohenlieth, Germany) and two partially resistant genotypes, B. napus SEM 05-500256 from Svalöf Weibull (Svalöf, Sweden) and B. oleracea genotype BRA 1008/01 from the Institute of Plant Genetics and Crop Plant Research, Gatersleben, Germany, were used in this study. Seeds were double surfacesterilized by sequential immersion in $70 \%$ ethanol for $2 \mathrm{~min}$ and $1 \%$ sodium hypochlorite containing $0.1 \%$ Tween-20 for 15 min under constant shaking (Zou et al., 2004). After washing the seeds twice with autoclaved tap water they were sown in double-autoclaved silica sand. Plantlets were grown in a climate chamber at $23 / 20^{\circ} \mathrm{C}$ (day/night) and 14 -h day length. For real-time PCR analyses, disease scoring and the histochemical studies, 14 days-old plants were transferred to an acclimation chamber and kept at $4{ }^{\circ} \mathrm{C}$ for ten weeks with light conditions as above to ensure vernalisation.

\section{Fungal isolate}

Verticillium longisporum isolate VL 43 from B. napus was used throughout this work. The isolate originates from diseased oilseed rape plants collected from a field in Northern Germany (Zeise \& von Tiedemann, 2001; Zeise \& von Tiedemann, 2002 a; Zeise \& von Tiedemann, $2002 \mathrm{~b}$ ). Long-term storage was performed as conidial suspension with 1 to $3 \mathrm{x}$ $10^{6}$ conidia $\mathrm{mL}^{-1}$ in Czapek Dox medium supplemented with $25 \%$ glycerol at $-80^{\circ} \mathrm{C}$. Inoculum for the artificial infection was produced by adding $500 \mu \mathrm{L}$ of the spore stock solution to $250 \mathrm{~mL}$ potato dextrose broth (PDB). The cultures were subsequently incubated for 7 days at $23^{\circ} \mathrm{C}$ on the rotary shaker. The resulting suspension was filtered through sterile gauze. Spore concentration was determined with a haemocytometer and adjusted to $1 \times 10^{6}$ spores $\mathrm{mL}^{-1}$ for inoculation.

Inoculation and growth conditions

In all experiments described in this report, seedlings were inoculated with the root-dipping method. After vernalisation and recovering for one week (real-time PCR analysis, assessment of disease development, histochemistry) or cultivation for fourteen days after germination (analysis of phenolic compounds), seedlings were carefully removed from the substrate and the roots gently washed under tap water. Inoculation was performed by dipping the intact roots in a spore suspension of $V$. longisporum isolate VL 43 for $30 \mathrm{~min}$. Control plants were 
dipped in sterilized tap water for the same time. Plantlets for real-time PCR analysis and disease phenotyping (symptom development, plant height and morphological features) were transferred into pots with a sand:soil (1:1) mixture and grown under standardised conditions at $20^{\circ} \mathrm{C}$ and $14 \mathrm{~h}$ day length. Plantlets for biochemical analyses were grown in the same substrate and kept in a climate chamber at $23 / 20^{\circ} \mathrm{C}$ (day/night) and 14-h day length (Philips TL5 HO lamps).

\section{Assessment of disease symptoms}

Disease symptoms were evaluated using an assessment key with nine classes, as described earlier (Eynck et al., 2007). Scoring was conducted at various time points from 16 to 79 days post inoculation (dpi). AUDPC values (area under the disease progress curve) were calculated from the disease severity values according to the following formula (Campbell \& Madden, 1990):

$$
\operatorname{AUDPC}=\sum_{i=1}^{n}\left(y_{i}+y_{i+1} / 2\right) *\left(t_{i+1}-t_{i}\right)
$$

where $y_{i}$ is the disease severity value for observation number $i$, $t i$ is the corresponding number of days post inoculation of an observation, and $n$ is the number of observations.

\section{DNA isolation and real-time PCR analysis}

In order to investigate the fungal spread in the plant, real-time PCR analysis was performed with the two oilseed rape genotypes 'Falcon' and SEM 05-500256. Ten inoculated and five control plants were harvested for real-time PCR analyses at five time points from 16 to 79 dpi. Samples were collected from the hypocotyl (below cotyledon node) and an above stem part (below basal leaf), resulting in twenty and ten tissue samples and PCR analyses for the treated and the control variant, respectively. Roots were not analysed because a proper discrimination between fungal biomass in the roots from fungus attached on the root surface through inoculation was not possible. DNA isolation and PCR analysis were performed as described previously (Eynck et al., 2007).

\section{Histological examinations}

For histological investigations all three genotypes were used. Sampling was conducted 21 days after infection on ten inoculated and ten healthy plants. From the hypocotyls a piece of 2 
$\mathrm{cm}$ in length was excised and preserved in a mixture of formalin, acetic acid and ethanol (AFE). For histochemical analyses, tissue samples were transverse cross-sectioned on a vibration microtome (Leica VT 100M, Leica, Bensheim, Germany), treated with histochemical reagents within $5 \mathrm{~min}$ and immediately examined with light or epifluorescence microscopy (Leica DMRB, Leica, Bensheim, Germany). Histochemical stains included toluidine blue (Feder \& O`Brien, 1968), phloroglucinol-HCl (Jensen, 1962), and FolinCiocalteu reagent (Singleton et al., 1999).

In order to detect vascular occlusions, transverse stem sections were treated with $0.05 \%$ toluidine blue in Aqua dest. After staining, vascular gels and tyloses exhibited a pink colour due to their pectic composition. The presence of polyphenolic compounds was determined with toluidine blue and the Folin-Ciocalteu reagent which induced a dark blue or brown to black colour, respectively. The stem sections (20 to $30 \mu \mathrm{m}$ thick) were mounted in water. Polyphenolic compounds were also detected by harnessing their autofluorescence under near UV light using the following filter set: $340-380 \mathrm{~nm}$ excitation filter, dichromatic mirror at 400 $\mathrm{nm}$ and longpass suppression filter at $425 \mathrm{~nm}$. Lignin was visualized with phloroglucinol-HCl (Wiesner test). Sections were immersed in phloroglucinol in $96 \%$ ethanol for $2 \mathrm{~min}$, rinsed with $32 \% \mathrm{HCl}$ and mounted in $\mathrm{HCl}(32 \%)$. After this procedure lignin compounds stain bright purple-red.

Analysis of soluble phenolic acids, cell wall-bound phenolics and lignin

Ten inoculated as well as control plants of all three genotypes were harvested for biochemical analysis at weekly intervals from 7 until $28 \mathrm{dpi}$. While plants harvested at 7 and 14 dpi were separated into roots and shoots, plants at 21 and 28 dpi were further divided into roots, hypocotyls and the youngest fully developed leaves. The total soluble and cell wall-bound phenolic content was determined with the Folin-Ciocalteau assay (Swain \& Hillis, 1959).

Quantification of soluble phenolic acids

Up to $200 \mathrm{mg}$ of plant tissue were extracted in $1.5 \mathrm{~mL}$ of $80 \%$ aqueous methanol at room temperature for $1 \mathrm{~h}$ and centrifuged $(13000 \mathrm{xg})$ for $10 \mathrm{~min}$ at $4^{\circ} \mathrm{C}$. The supernatant was saved and the process repeated once. After extraction, the supernatants were merged and used for the Folin-Ciocalteau assay (modified from Pritchard et al., 1997) as follows: $100 \mu \mathrm{L}$ of extract were added to $100 \mu \mathrm{L}$ distilled water and $200 \mu \mathrm{L}$ of Folin-Ciocalteau reagent. After an incubation of $3 \mathrm{~min}$ at room temperature, $200 \mu \mathrm{L}$ of $1 \mathrm{M} \mathrm{Na}_{2} \mathrm{CO}_{3}$ were added. After further incubation on a rotary shaker for $45 \mathrm{~min}$ at room temperature, the absorbance of the samples 
was measured at $725 \mathrm{~nm}$. Tannin was used as an external standard, and total soluble phenolic content was reported as tannin equivalents based on a calibration curve. Aliquots of the particular samples were frozen at $-20^{\circ} \mathrm{C}$ for HPLC analysis.

Quantification of cell wall-bound phenolic acids

For quantification of the cell wall-bound phenolic acids, a method described by Strack et al. (1988) was used. After thoroughly washing the remaining pellets from the extraction of the free phenolic acids (see above), $1 \mathrm{~mL}$ of $1 \mathrm{M} \mathrm{NaOH}$ was added and the samples incubated for one hour at $80^{\circ} \mathrm{C}$ and another $12 \mathrm{~h}$ at room temperature (alkaline hydrolysis). Subsequently the samples were acidified to $\mathrm{pH}<4.0$ with $100 \mu \mathrm{L} 86 \% \mathrm{H}_{3} \mathrm{PO}_{4}$. Ethyl acetate $(500 \mu \mathrm{L})$ was added and after incubating on a rotary shaker for $30 \mathrm{~min}$, the samples were centrifuged $(13000 \times \mathrm{g}$ ) for $5 \mathrm{~min}$. After collecting the upper phase, the lower phase was extracted again with ethyl acetate. The combined ethyl acetate phases were then fully evaporated. The residue pellets obtained were resuspended in $500 \mu \mathrm{L}$ methanol and used for further quantitative and qualitative analysis. Thus, one part of the samples was analysed using the Folin-Ciocaleau method as described above and the other was frozen at $-20^{\circ} \mathrm{C}$ for HPLC analysis.

\section{Lignin analysis}

Lignin was analysed with the thioglycolic acid procedure according to Bruce \& West (1989), modified by Otter (1996). After washing the pellet of the alkaline hydrolysis was treated with $1.5 \mathrm{~mL} 2 \mathrm{M} \mathrm{HCl}$ and $0.3 \mathrm{~mL}$ thioglycolic acid for $4 \mathrm{~h}$ at $94^{\circ} \mathrm{C}$. After short cooling, the pellets were collected by centrifugation, washed two times with distilled water and the residues incubated with $1 \mathrm{~mL} 0.5 \mathrm{M} \mathrm{NaOH}$ for $12 \mathrm{~h}$. Subsequently, the suspension was centrifuged, extracted again with $0.5 \mathrm{~mL} 0.5 \mathrm{M} \mathrm{NaOH}$ and centrifuged again. The corresponding supernatants were merged and acidified with $300 \mu \mathrm{L}$ of $32 \% \mathrm{HCl}$ to precipitate the lignothioglycolic acid complex. After $4 \mathrm{~h}$ incubation at $4^{\circ} \mathrm{C}$ on a rotary shaker, the precipitates were collected by centrifugation and the pellets were resuspended in $2 \mathrm{~mL}$ of $0.5 \mathrm{M} \mathrm{NaOH}$. Finally, the absorbance of the samples was measured at $280 \mathrm{~nm}$ after adequate dilution with $0.5 \mathrm{M} \mathrm{NaOH}$. The assay was calibrated with alkaline lignin.

\section{Statistical analysis}

Results are given as means \pm standard deviation of ten replicates. Statistical analyses were conducted using the computer software StatGraphics. Differences among means were tested with Fisher's least significant difference (LSD) test and significances determined at $p \leq 0.05$. 


\section{Results}

Progression of disease

Sixteen days after inoculation infected plants of the susceptible cultivar 'Falcon' showed first disease symptoms, namely yellowing and the occurrence of black veins on the oldest leaves, whereas no visible symptoms appeared on leaves of accession SEM 05-500256 (Table 1). At the same time, both genotypes exhibited significant stunted growth upon inoculation (Table 2). While at 30 dpi only a few plants of SEM 05-500256 showed faint symptoms on the first true leaves, cultivar 'Falcon' exhibited severe symptoms with $90 \%$ of the plants having $50 \%$ or more damaged leaves. Again a significant reduction in plant height was visible in both genotypes. Until 79 dpi, the disease progressed in 'Falcon' and until the end of the experiment all plants developed severe disease symptoms. Concomitantly, plant growth was impaired during this time period in the infected plants, which was most obvious at 51 dpi and tended to be partially compensated until 79 dpi. In contrast, SEM 05-500256 plants looked similar to the non-inoculated control plants showing only weak symptoms (yellowing) on the oldest leaves and no significant stunting. 


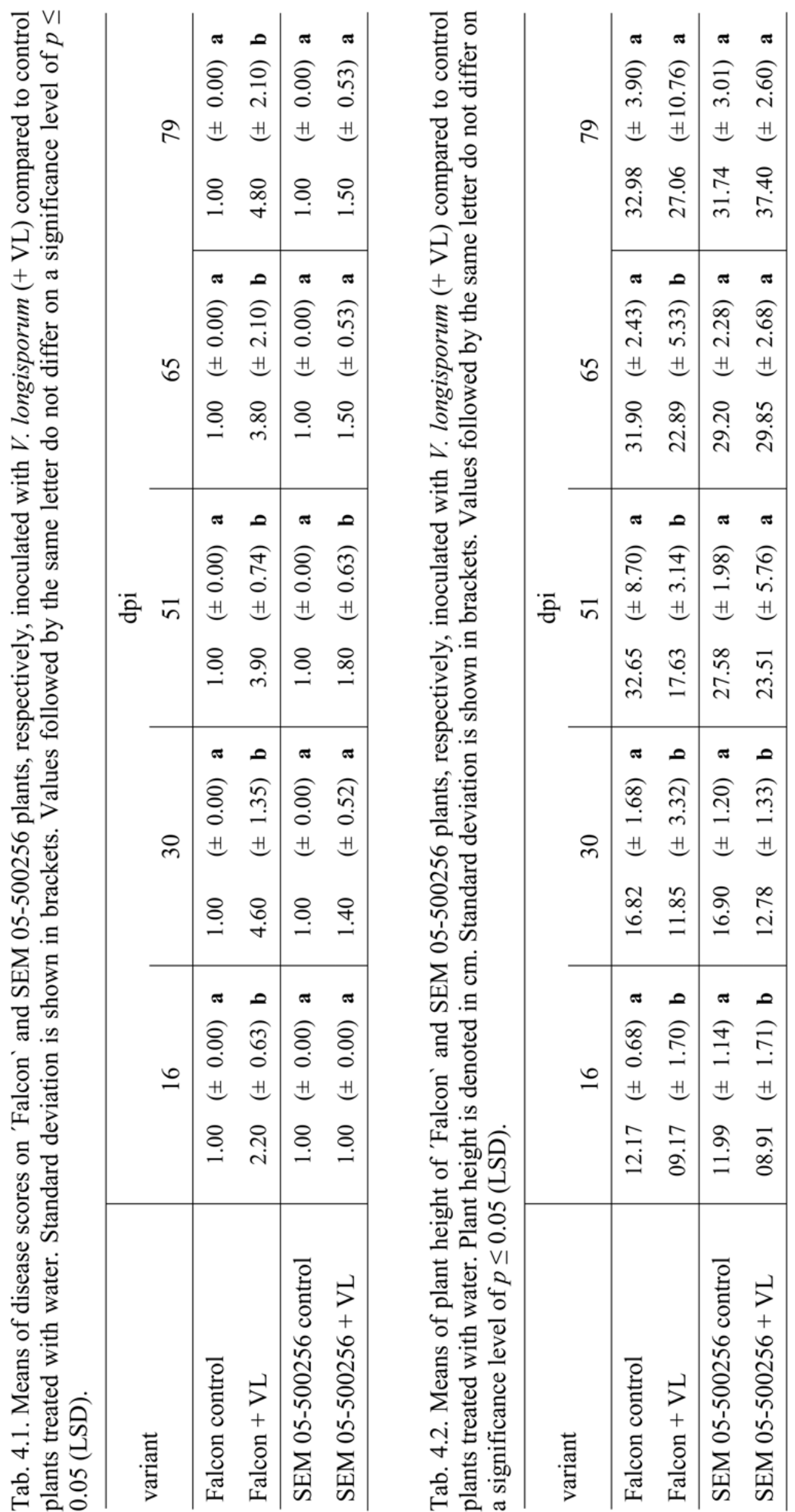


Fungal growth in the plant

At 16 dpi, V. longisporum occurred in both 'Falcon' and SEM 05-500256 in about equal amounts but was restricted to the hypocotyls. Only at 30 dpi Verticillium DNA was detectable in the upper plant parts, however, displaying substantially higher amounts in the susceptible cultivar (Figure 4.1). The amount of V. longisporum further rose in 'Falcon' in both tissue types transiently arrested at $65 \mathrm{dpi}$ in the hypocotyl. At 79 dpi there was twice as much Verticillium DNA in the upper stem parts (203.45 ng/g fresh weight) than in tissues from the plant base (120.35 ng/g FW). By contrast, the amount of Verticillium in stems of SEM 05500256 was substantially lower than in the susceptible cultivar, and never exceeded $25.0 \mathrm{ng} / \mathrm{g}$ $\mathrm{FW}$ in the hypocotyl or about $5 \mathrm{ng} / \mathrm{g} \mathrm{FW}$ in the upper stem.

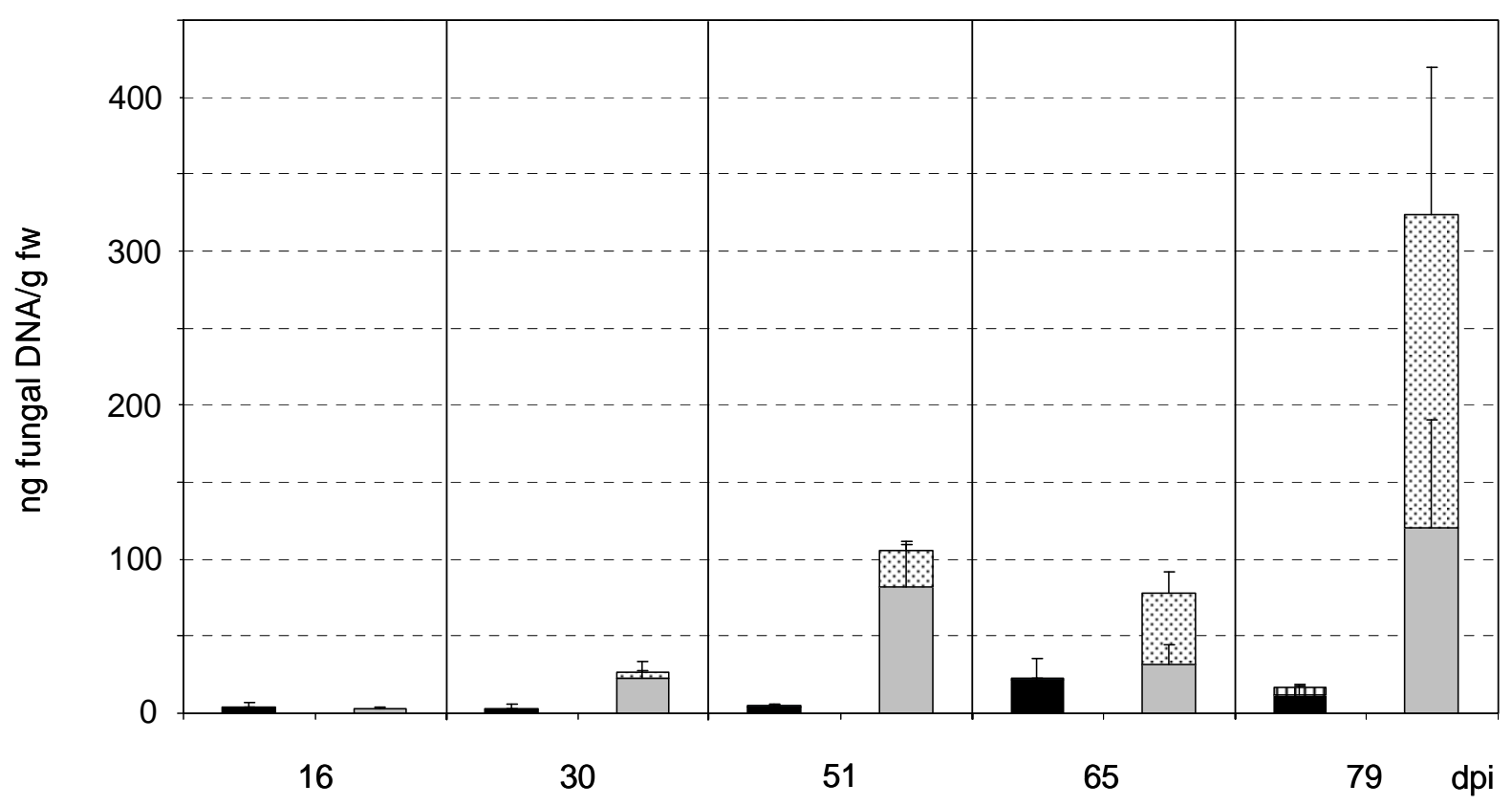

Fig. 4.1. Amount of Verticillium DNA detected with real-time PCR in tissue samples (means of fungal DNA in lower and upper stem parts \pm standard deviation) of B. napus cv. 'Falcon' (right bars) and SEM 05-500256 (left bars) inoculated with $V$. longisporum. Lower stem part of SEM 05-500256: black bars; upper stem part of SEM 05-500256: dashed bars; lower stem part of Falcon: grey bars; upper stem part of Falcon: dotted bars.

Histological features of tissue from healthy plants

There were no differences in the histology of healthy susceptible and resistant plant hypocotyls with regard to the staining of cell walls after treatment of sections with toluidine blue, Folin-Ciocalteu reagent or after observation under fluorescent light, the latter revealing that a background autofluorescence was a general feature of healthy hypocotyl tissue. 
However, after treatment with phloroglucinol-HCl, hypocotyl sections from resistant control plants exhibited a stronger staining of xylem vessel walls and parenchyma cell walls.

Histological responses in Verticillium-infected B. napus hypocotyls

Twenty-one days after inoculation some anatomical changes associated with the infection with $V$. longisporum were visible in the hypocotyls (Figure 4.2 to 4.8 ). Thus, after treatment with toluidine blue, occlusions were noticeable in the xylem vessels of all Brassica varieties (Figure 4.2 B, D, F), the resistant SEM 05-500256 (Figure 4.2 D) featuring a higher number of occluded vessels than the susceptible 'Falcon` (Figure 4.2 B). BRA 1008/01 showed by far the highest proportion of occluded vessels (Figure 4.2 F; Figure 4.3 F). The majority of these obstructions appeared in vessels located near the centre of the hypocotyl transverse section. Furthermore, most of the vascular occlusions stained pink with toluidine blue, indicating that they originate from primary cell wall material (Gerlach, 1984). Some of the vessel plugs exhibited a faint blue colour (e.g. Figure 4.2 D) which might be an indication for beginning infusion of phenolic compounds into the plant material. Only in the cabbage genotype, some obstructions produced a black staining, clearly indicating an impregnation with phenolic material (Figure 4.2 F; Figure 4.3 F). Staining with toluidine blue also revealed structurally unmodified parenchyma cells adjacent to xylem vessels which were filled with material stained dark-blue or black (Figure 4.2 B, D, F; Figure 4.3 B, D, F). After sections were stained with Folin-Ciocalteu reagent, microscopic analysis likewise showed that xylem parenchyma cells in close proximity to xylem vessels exhibited a dark discoloration after infection with $V$. longisporum, indicating the presence of phenolic compounds (Figure $4.4 \mathrm{~B}$ D, F). Although for all genotypes the occurrence of these so-called phenolic-storing cells (Beckman, 2000) was observed, their distribution in the host tissue and the accumulation of phenolic compounds differed. While in the susceptible cultivar phenolic-storing cells only occurred around vessels located in the hypocotyl centre, the resistant varieties showed darkstained paravascular contact cells all over the cross section area. Furthermore, xylem vessels of the resistant genotypes were surrounded by sheath cells with a more pronounced and stronger staining (Figure 4.4 D, F) than in susceptible plants where phenolics appeared less concentrated around vessels and more diffusively scattered over the section area (Figure 4.4 B).

The use of the Wiesner test revealed red-stained xylem vessel walls as well as parenchyma cell walls (Figure 4.5; Figure 4.6). After infection all genotypes exhibited a stronger colouration of vessel walls and walls of adjacent cells as compared to other parenchyma cells 
(Figure 4.5 B, D, F; Figure 4.6 B, D, F). Thus, lignification appeared to occur predominantly in the vascular tissue. Nevertheless, vessel elements of the susceptible genotype stained weaker for lignin than the corresponding cells from the resistant accessions. Similarly, a positive reaction was detected with material that plugged xylem vessels mainly in the centre region of the hypocotyl cross-sections, a reaction that was strongly developed in the susceptible 'Falcon' (Figure 4.5 B; Figure 4.7 C). Thus, xylem vessels of both infected susceptible and resistant plants were plugged by pectic and lignin-like material.

Observations on semi-thin sections under near UV illumination (Figure 4.8) revealed autofluorescence to be much stronger in lignified xylem areas of infected plants (Figure 4.8 B, D) in comparison to healthy plants (Figure $4.8 \mathrm{~A}, \mathrm{C}$ ). However, this reaction occurred at a significantly higher intensity in the resistant plants (Figure 4.8 D). This enhanced autofluorescence indicating the presence of phenolic compounds was observed both in plugging material filling vessel elements and the paravascular contact cells. 

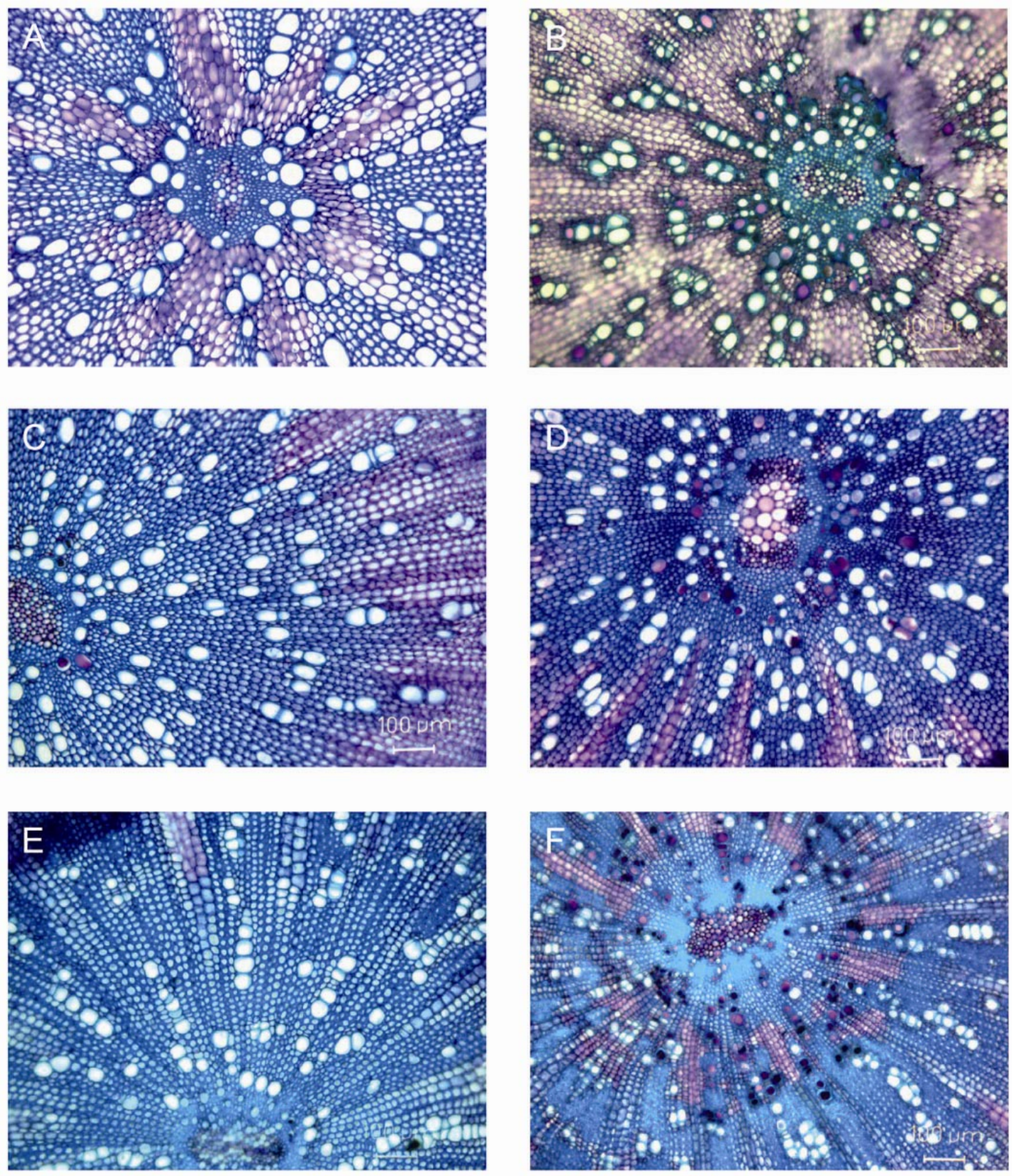

Fig. 4.2. Light microscopy images of hypocotyl cross sections of cultivar 'Falcon', B. napus accession SEM 05-500256 and B. oleracea accession BRA 1008/01 after staining with toluidine blue for detection of occlusions and phenolic substances in control plants and after infection by $V$. longisporum (21 dpi). A, 'Falcon', control; xylem vessels completely free of obstructions. B, 'Falcon', infected; occlusion of vessels particularly in the centre region due to purple to pink stained substances (tyloses, vascular gels). Dark staining of xylem parenchyma cells indicates an accumulation of phenolic substances. C, SEM 05-500256, control plants; infrequent closure of single vessels can be observed. D, SEM 05-500256, infected; considerably more vessels are blocked than in $\mathbf{B}$ (purple to pink colouration of accumulated material). E, BRA 1008/01, control; no obstructions visible, already control plants exhibit substantially thicker cell walls. F, BRA 1008/01, infected; a high percentage of vessels is blocked with pink material, some of the obstructions stain black, indicating that phenolic substances have been infused. Bars represent $100 \mu \mathrm{m}$. 

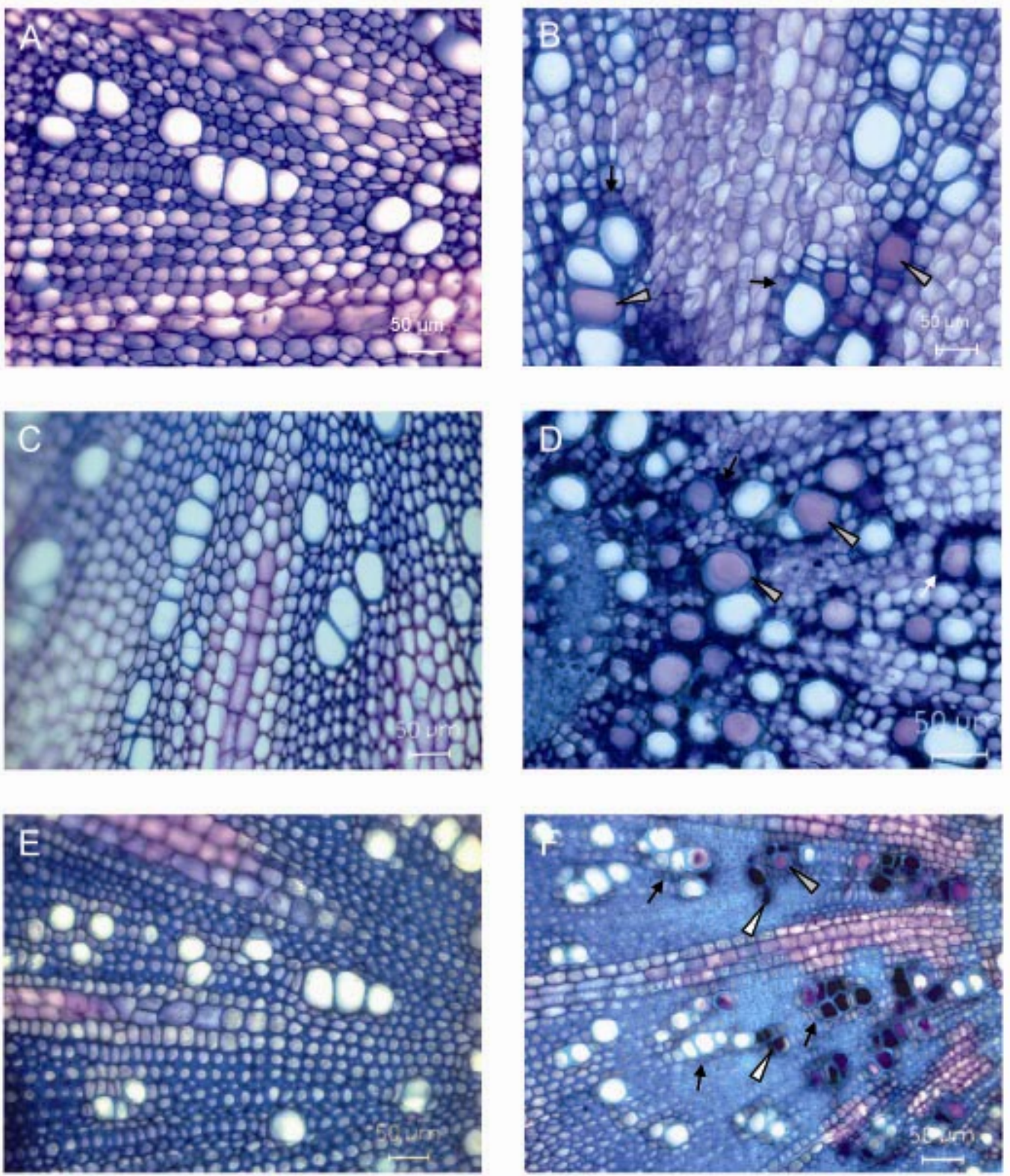

Fig. 4.3. Light microscopy images of hypocotyl cross sections of cultivar 'Falcon', B. napus accession SEM 05-500256 and B. oleracea accession BRA 1008/01 after staining with toluidine blue for detection of occlusions and phenolic substances in control plants and after infection by $V$. longisporum (21 dpi). Same coherences as in $\mathbf{A}$ to $\mathbf{F}$ in Figure 4.1., depicted at a higher magnification (see bars). A, 'Falcon', control; xylem vessels completely free of obstructions. B, 'Falcon', infected; dark colouration (arrow) indicates a pronounced accumulation of phenolics in xylem parenchyma cells. Arrowheads highlight obstructed vessels. C, SEM 05-500256, control plants; vessels and surrounding xylem parenchyma completely free and clear, respectively. D, SEM 05-500256, infected; the pronounced dark colouration of cells directly neighbouring the xylem vessels is a sign of massive accumulation of phenols. E, BRA 1008/01, control; dark (grey) background staining of the tissue indicates that this genotype exhibits a higher constitutive level of phenolics. F, BRA 1008/01, infected; strong accumulation of phenolic substances in xylem parenchyma (arrow). Grey arrowhead: occlusion of vessels due to tyloses or vascular gels (pink staining). White arrowhead: deposition of phenolic substances in blocking material leads to a black staining. Bars represent $50 \mu \mathrm{m}$. 

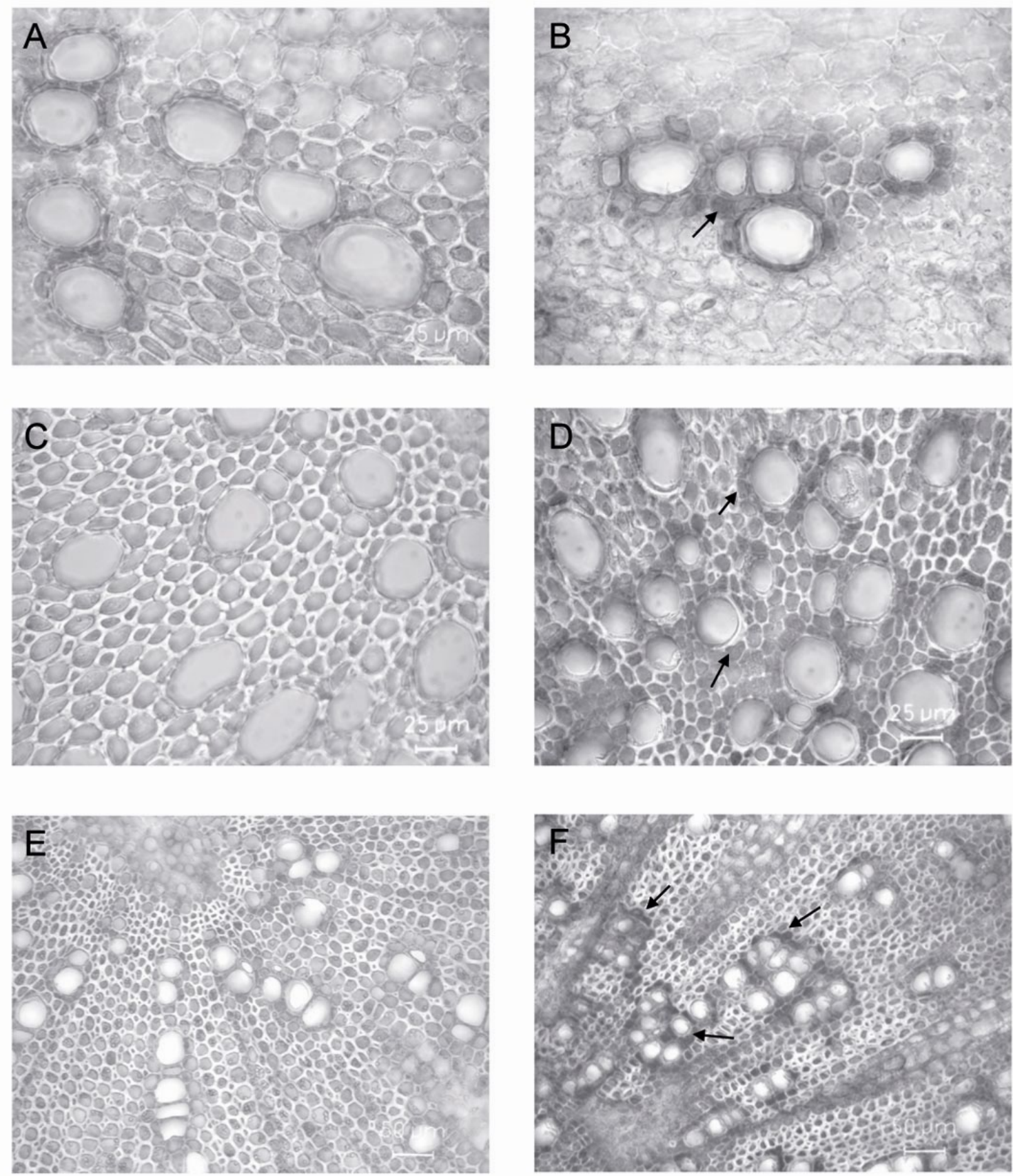

Fig. 4.4. Light microscopy images of hypocotyl cross sections of cultivar 'Falcon', B. napus accession SEM 05-500256 and B. oleracea accession BRA 1008/01 after staining with FolinCiocalteau reagent for detection of phenolic substances in control plants and after infection by V. longisporum (21 dpi). A, 'Falcon', control. B, 'Falcon', infected; accumulation of phenolic substances in xylem parenchyma cells (arrows). C, SEM 05-500256, control. D, SEM 05500256, infected; strong accumulation of phenolic substances is restricted to cells directly neighbouring xylem vessels (arrows). E, BRA 1008/01, control. F, BRA 1008/01, infected; massive accumulation of phenolic substances in cells surrounding the xylem (arrows). Bars in $\mathbf{A}-\mathbf{D}$ represent $25 \mu \mathrm{m}$, in $\mathbf{E}$ and $\mathbf{F} 50 \mu \mathrm{m}$. 

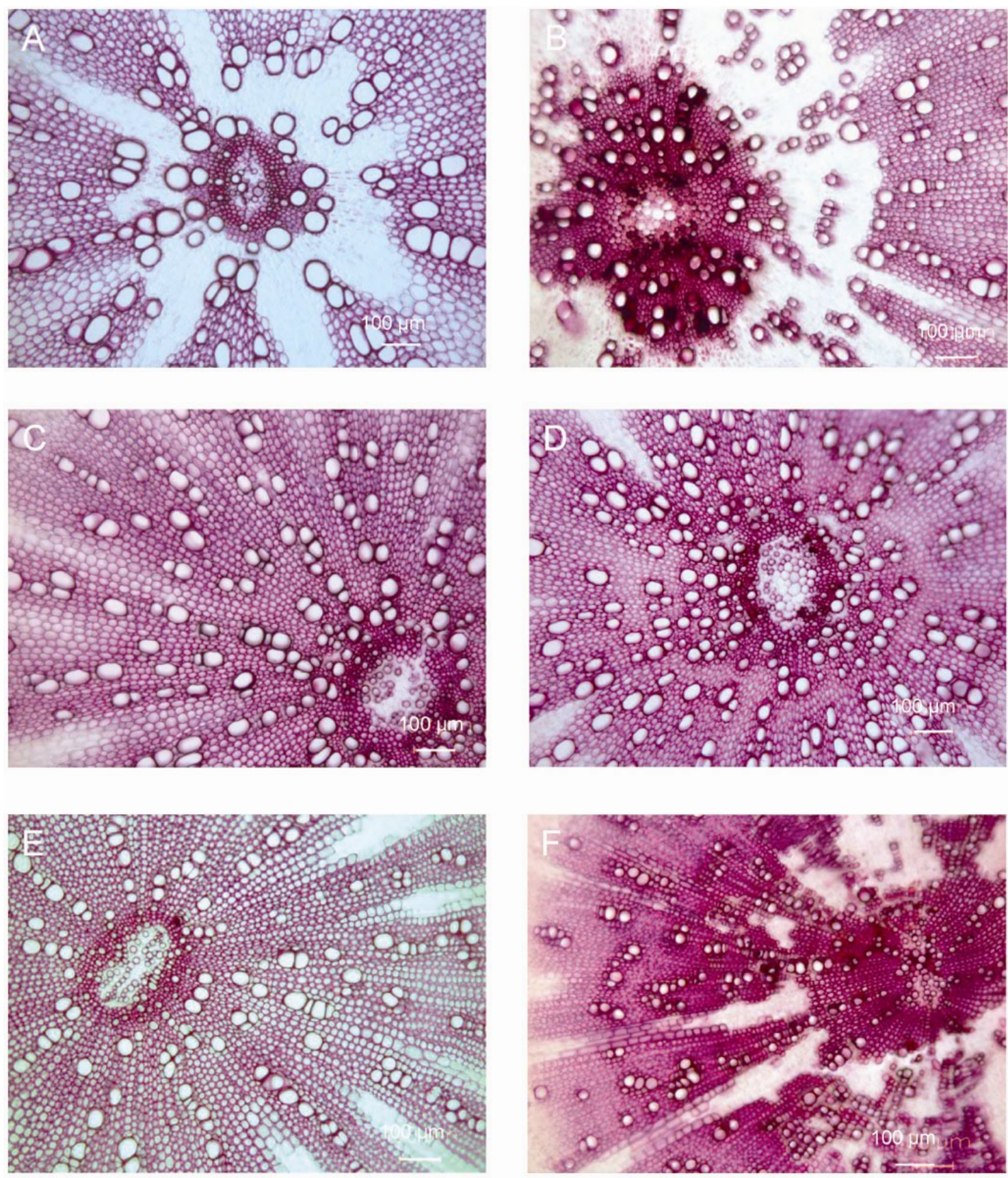

Fig. 4.5. Light microscopy images of hypocotyl cross sections of cultivar 'Falcon', B. napus accession SEM 05-500256 and B. oleracea accession BRA 1008/01 after staining with phloroglucinol- $\mathrm{HCl}$ for detection of lignin in control plants and after infection by $V$. longisporum (21 dpi). A, 'Falcon', control. B, 'Falcon', infected; massive lignification in the centre region of hypocotyl cross sectional area as well as occlusion of vessels. In border areas only a weak reaction could be detected. C, SEM 05-500256, control. D, SEM 05-500256, infected; increased lignification over the whole cross section, particularly of vessels and xylem parenchyma. E, BRA 1008/01, control. F, BRA 1008/01, infected; massive lignification over the complete cross sectional area, strongly thickened cell walls and obstruction of vessels. Bars represent $100 \mu \mathrm{m}$. 

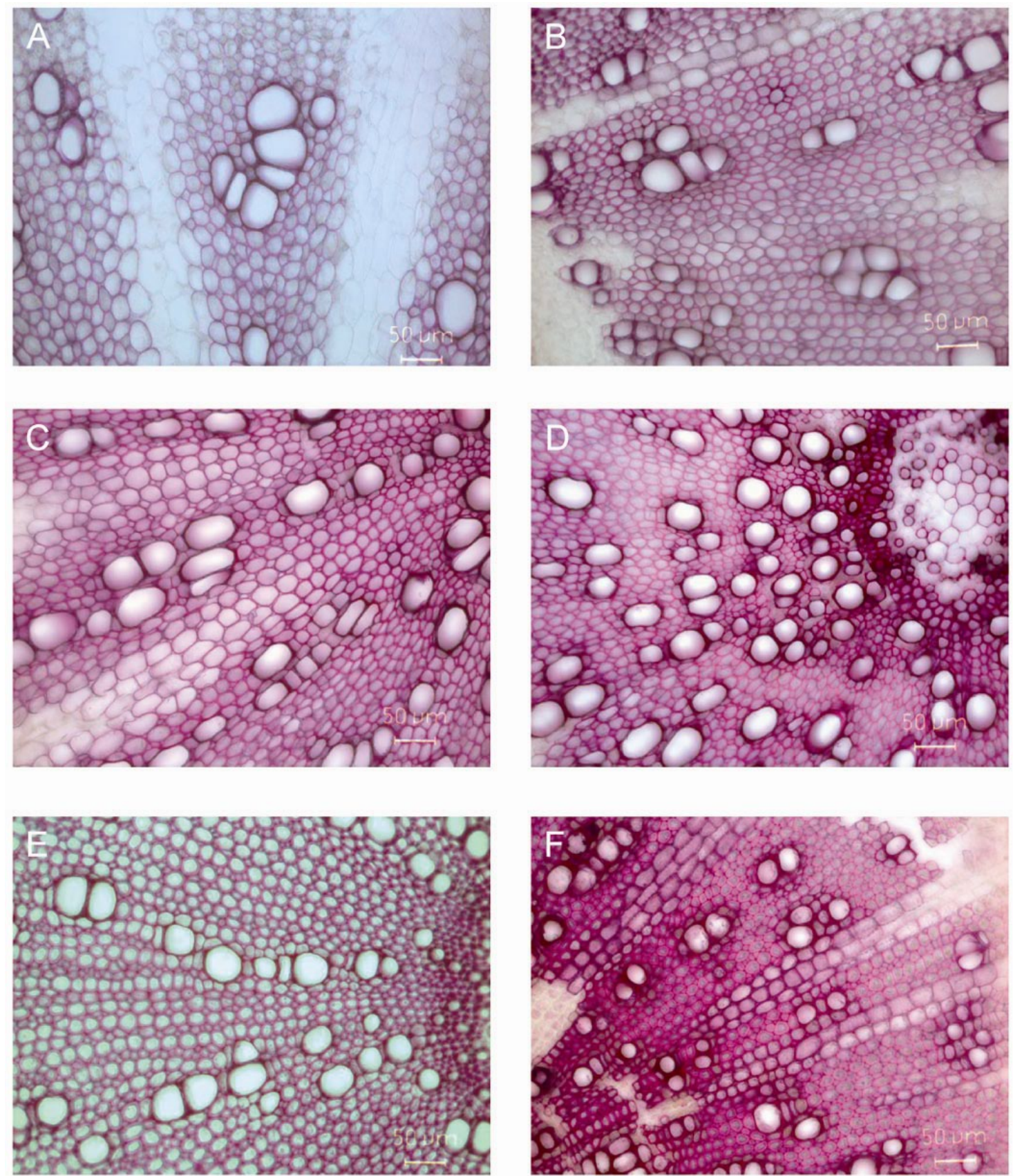

Fig. 4.6. Light microscopy images of hypocotyl cross sections of cultivar 'Falcon', B. napus accession SEM 05-500256 and B. oleracea accession BRA 1008/01 after staining with phloroglucinol-HCl for detection of lignin in control plants and after infection by $V$. longisporum (21 dpi). Same coherences as in $\mathbf{A}$ to $\mathbf{F}$ in Figure 4.4., depicted at a higher magnification (see bars). A, 'Falcon', control. B, 'Falcon', infected; sparsely reinforced lignification of vessel elements and surrounding xylem parenchyma cells. C, SEM 05500256, control. D, SEM 05-500256, infected; increased lignification particularly of xylem vessels and surrounding parenchyma cells. E, BRA 1008/01, control; F, BRA 1008/01, infected; massive deposition of lignin particularly in vessel walls and walls of adjacent xylem parenchyma cells. Bars represent $50 \mu \mathrm{m}$. 

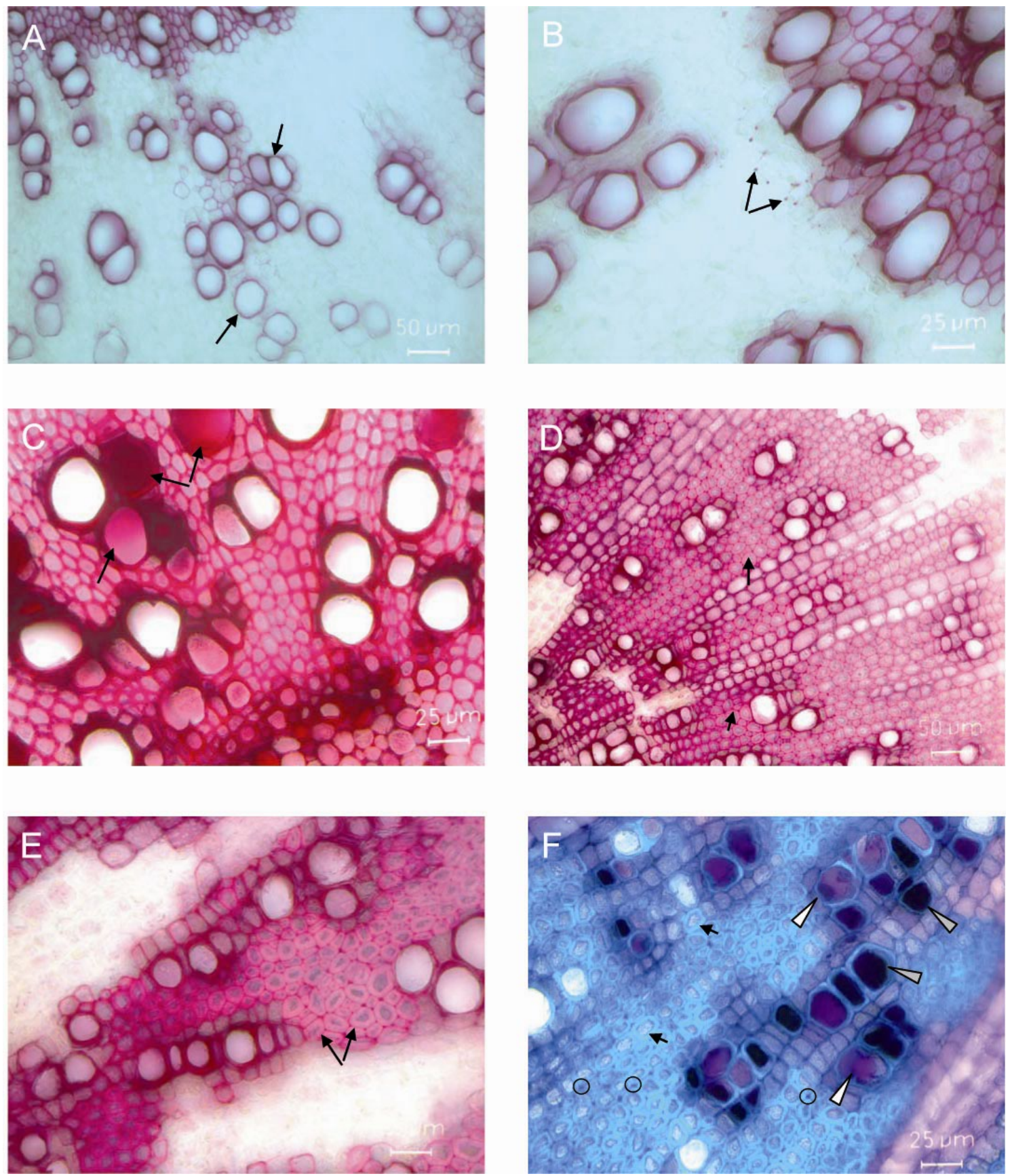

Fig. 4.7. Light microscopy images of hypocotyl cross sections of cultivar 'Falcon', B. napus accession SEM 05-500256 and B. oleracea accession BRA 1008/01 after staining with phloroglucinol- $\mathrm{HCl}$ for detection of lignin after infection by $V$. longisporum, 21 dpi (A - E) as well as toluidine blue for the detection of vascular occlusions and phenolic substances $(\mathbf{F})$. A, SEM 05-500256, lignin deposition in cell walls of xylem vessels and surrounding xylem parenchyma cells (arrows). B, SEM 05-500256, lignification of middle lamella starts in intercellular spaces (arrows). C, Falcon, strong lignification of cell walls in the centre of the cross sectional area and blocking of vessels by lignin-like material (arrows). D, BRA 1008/01, strong thickening of cell walls also in parenchyma cells due lignin deposition (arrows). E, BRA 1008/01, distinct middle lamellae (arrows). F, BRA 1008/01, strong thickened cell walls (arrows), occlusion of vessels due to tyloses, vascular gels (white arrow heads) and deposition of phenolic substances into the blocking material (grey arrow heads), beginning lignification in intercellular spaces is marked with circles. Bars represent $50 \mu \mathrm{m}$ in $\mathbf{A}$ and $25 \mu \mathrm{m}$ in $\mathbf{B}-\mathbf{F}$. 

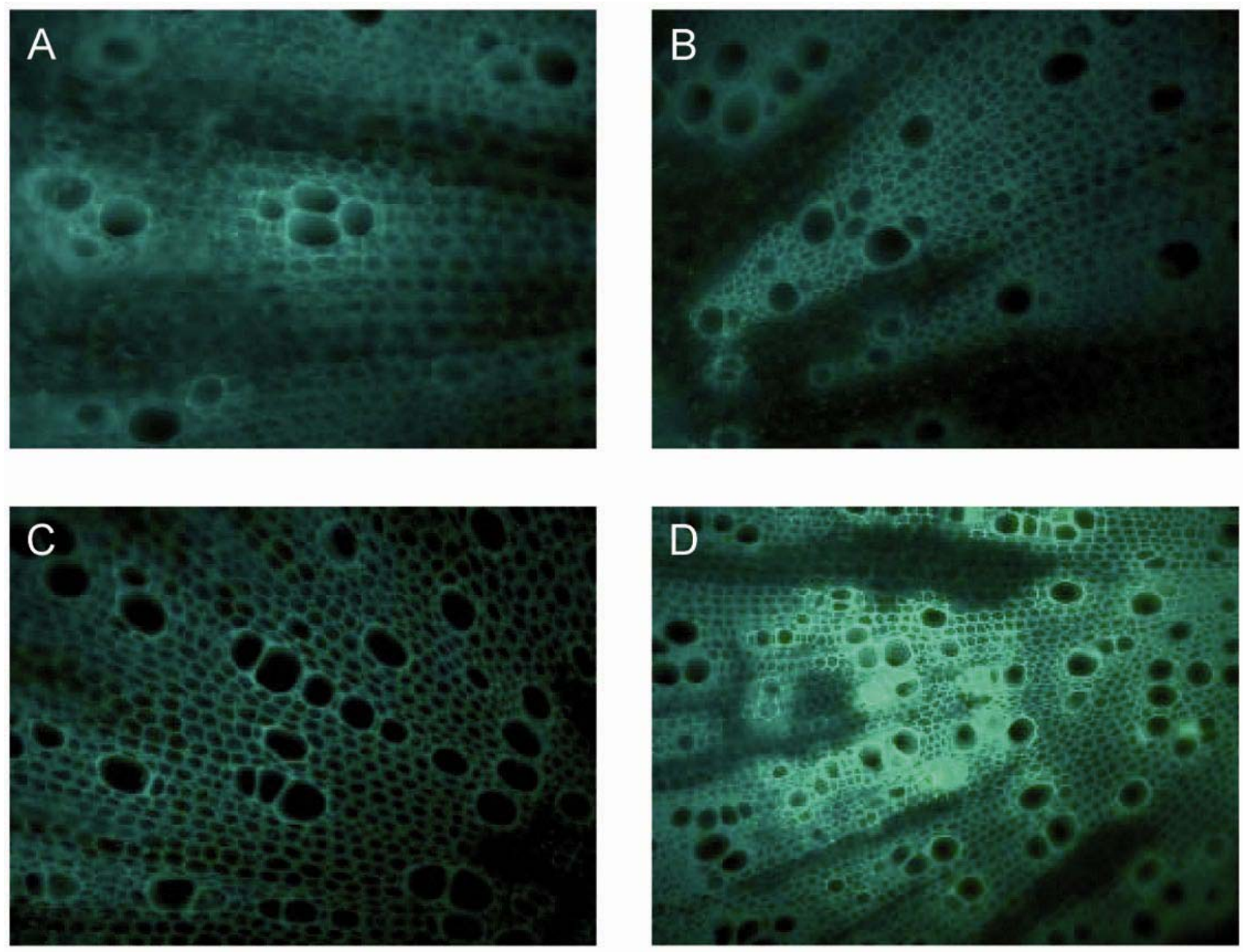

Fig. 4.8. Epifluorescence microscope images of hypocotyl cross sections of cultivar 'Falcon' (A, B) and B. napus accession SEM 05-500256 (C, D). Documentation of autofluorescence in the near-UV range typical for phenolics in control plants $(\mathbf{A}, \mathbf{C})$ and after infection with $V$. longisporum (B, D; 21 dpi). The resistant genotype SEM 05-500256 shows by far a stronger accumulation of phenolic substances than the susceptible genotype 'Falcon'; 200fold magnification.

Quantitative analysis of soluble phenolics, cell wall-bound phenolic acids and lignin

Soluble phenolic acids

At 7 dpi both the resistant $B$. napus and B. oleracea accessions possessed significantly higher amounts of soluble phenolic acids in both roots and shoots (Table 4.3), BRA 1008/01 exhibiting even higher amounts than SEM 05-500256. While in 'Falcon' the content of soluble phenolics decreased upon infection, both SEM 05-500256 and BRA 1008/01 showed a significant induction in the roots of inoculated compared to control plants. Furthermore, BRA 1008/01 exhibited a clear induction of phenols in the shoot. At 14 dpi, the level of phenolic acids in roots and shoots was substantially higher in control plants of the resistant genotypes in comparison to the susceptible genotype. However, while SEM 05-500256 exhibited a significant rise of the phenolic content in both roots and shoots after infection, the 
cabbage genotype did not show a significant difference between the control and inoculated variant and susceptible plants revealed an increase only in the shoot tissue. Along the lines of the controls, the amounts of free phenolic acids in the infected variant were considerably higher in the resistant $B$. napus variety $(6.1 \mathrm{mg} / \mathrm{g}$ dry weight (roots) and $11.1 \mathrm{mg} / \mathrm{g} \mathrm{dw}$ (shoots) in SEM 05-500256 and $4.5 \mathrm{mg} / \mathrm{g} \mathrm{dw}$ (roots) and $12.7 \mathrm{mg} / \mathrm{g}$ dw (shoots) in BRA $1008 / 01$ compared to $3.8 \mathrm{mg} / \mathrm{g} \mathrm{dw}$ (roots) and $8.2 \mathrm{mg} / \mathrm{g} \mathrm{dw}$ (shoots), respectively. Three weeks after infection, in the susceptible cultivar 'Falcon', no significant differences in the amount of phenolic acids between both roots and shoots of infected and control plants were detectable. Only hypocotyls of infected plants exhibited a slightly higher amount of soluble phenolics than the control. In contrast, the resistant SEM 05-500256 exhibited a considerably higher level of phenolics in all investigated tissues after infection, with the non-inoculated plants featuring values similar to 'Falcon' control plants. The cabbage variety showed a higher amount of soluble phenolics only in infected roots and hypocotyls, the particular value being on the same level as the corresponding values of SEM 05-500256 for the roots and 'Falcon' for the hypocotyls. The total amount of soluble phenolics in leaves of control and infected plants of BRA 1008/01 exceeded by far the corresponding amounts of the B. napus genotypes. Within one week, thus at $28 \mathrm{dpi}$, the total amount of soluble phenolics decreased or remained at the same level in the roots as well as the hypocotyls of both genotypes.

\section{Cell wall-bound phenolic acids}

At $7 \mathrm{dpi}$, the content of cell wall-bound phenolic acids clearly decreased in infected plants of the susceptible cultivar 'Falcon' compared to control plants (Table 4.4). This is in contrast to the resistant genotypes SEM 05-500256 and BRA 1008/01, displaying no significant differences between the infected and control shoot (SEM 05-500256) and root (BRA 1008/01) tissues, however, both showing significantly higher contents than 'Falcon'. Surprisingly, shoots in control cabbage plants exhibited the lowest amounts of bound phenolics. However the particular infected tissue samples featured values similar to the corresponding samples of oilseed rape. Analysis of 'Falcon' plants sampled at 14 dpi again revealed no difference concerning the amount of wall-bound phenolics in inoculated versus control plants. Wallbound phenolics in roots of SEM 05-500256 were twice as high as in Falcon roots, however, they were unchanged due to the inoculation. In contrast, infection of shoots induced a significantly higher amount of phenolics in the resistant genotype. No difference due to infection was observed in shoots of BRA 1008/01, but in infected roots, which exhibited values similar to the resistant $B$. napus genotype, and showed a significantly higher amount in 
comparison to controls. At $21 \mathrm{dpi}$, all examined tissues of the resistant B. napus genotype showed a substantially higher level of wall-bound phenolic acids in the infected variant compared to the control. In the susceptible cultivar, this was only true for hypocotyls and leaves, while the roots showed a lower level of phenolics due to infection. Similar results were observed for BRA 1008/01. The accumulation of cell wall-bound phenolic acids was most pronounced in the hypocotyls of all three genotypes as was already implied by the histochemical studies. Thus, cell walls of hypocotyls from infected plants contained $27 \%$ (susceptible oilseed rape) or 55\% (resistant oilseed rape) more ester-bound phenolics than controls. At 28 dpi, wall-bound phenolics in infected plants of the susceptible cultivar were lower in roots and leaves but remained elevated in the hypocotyls compared to the control. In comparison, roots of SEM 05-500256 showed similar levels of bound phenolic acids and no changes due to infection, while hypocotyls and leaves displayed a significantly higher amount in the infected variants. BRA 1008/01 exhibited equal amounts of esterified phenolic acids in roots and leaves and a significant induction in hypocotyls.

\section{Lignin content}

Until one week after infection, there were no changes in lignin content in the root tissue of all three genotypes (Table 4.5). However, the resistant genotypes possessed a higher constitutive level of total lignin. While the amount of lignin decreased in the shoots of the infected susceptible genotype, it significantly rose in the shoots of the corresponding resistant plants (4.8 $\mathrm{mg} / \mathrm{g} \mathrm{dw}$ compared to $3.5 \mathrm{mg} / \mathrm{g} \mathrm{dw}$ in SEM 05-500256 and $3.9 \mathrm{mg} / \mathrm{g}$ dw compared to $2.8 \mathrm{mg} / \mathrm{g} \mathrm{dw}$ in BRA 1008/01). From 14 to $28 \mathrm{dpi}$, lignin contents in roots and shoots were substantially higher in control and infected plants of the resistant genotypes. This was most pronounced at $21 \mathrm{dpi}$ in the hypocotyl tissue. Thus, cell walls of infected susceptible or resistant $B$. napus genotypes contained about $30 \%$ or $70 \%$ more lignin than the controls, respectively. At $28 \mathrm{dpi}$, infection decreased lignin contents in roots of susceptible plants, while it increased lignin in roots of SEM 05-500256 and BRA 1008/01. At this time point, no effect of infection was found in the hypocotyls of susceptible plants, but in the resistant varieties a significantly higher level of lignin was detectable in infected plants. 


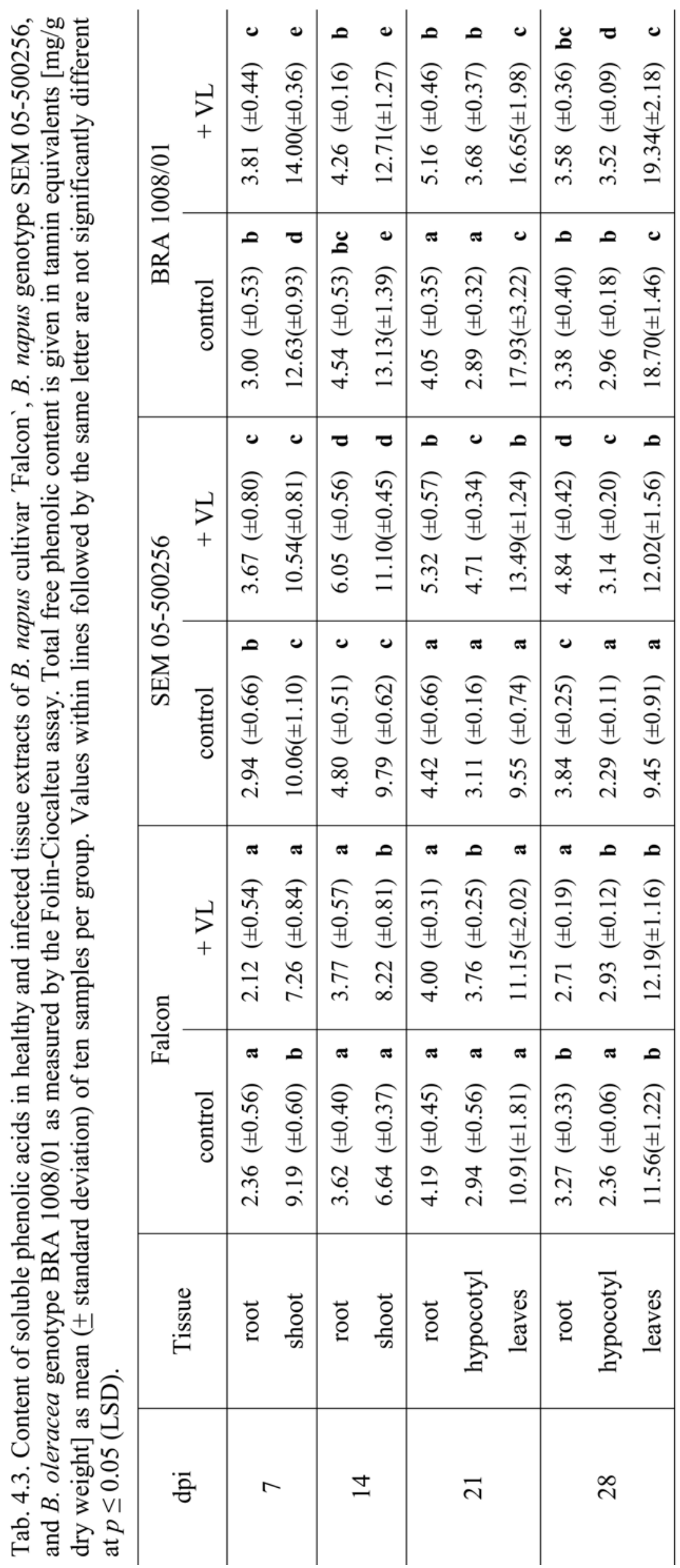




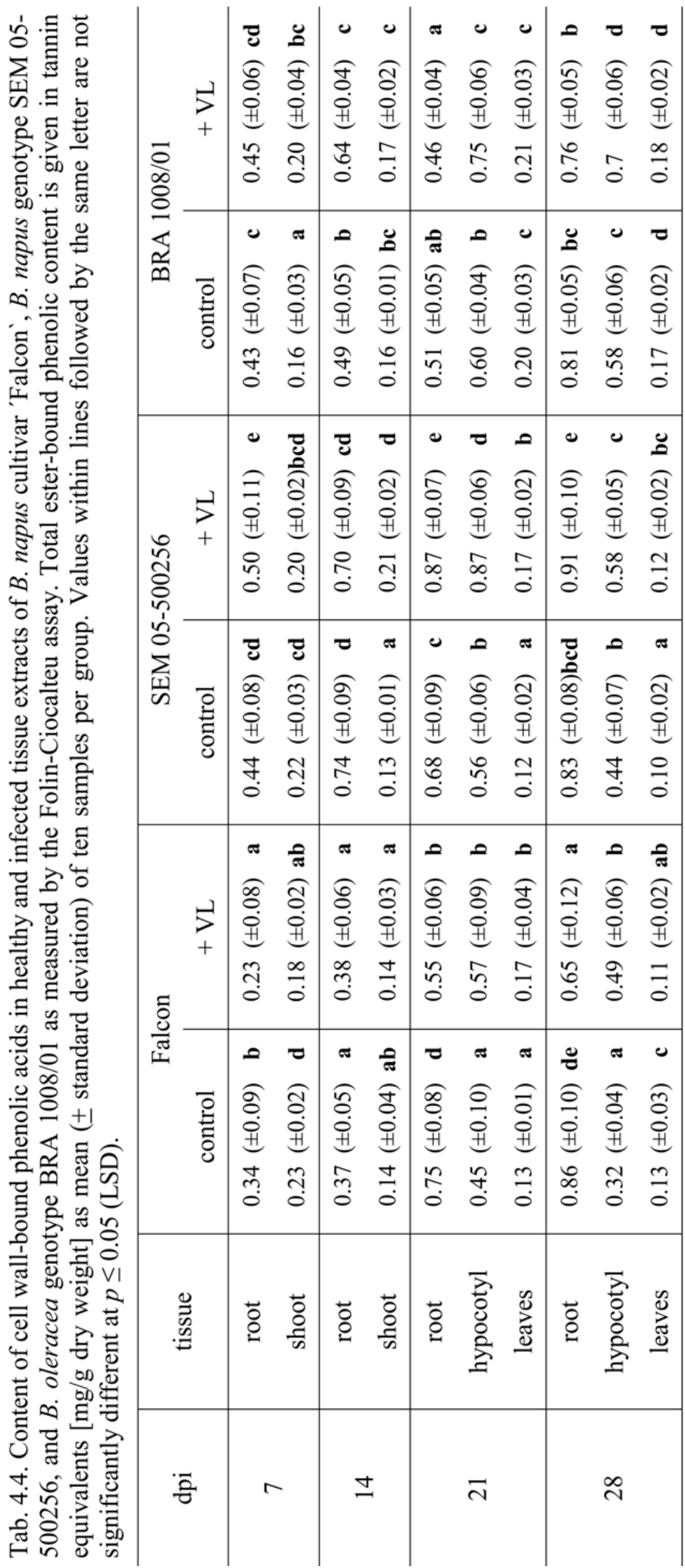




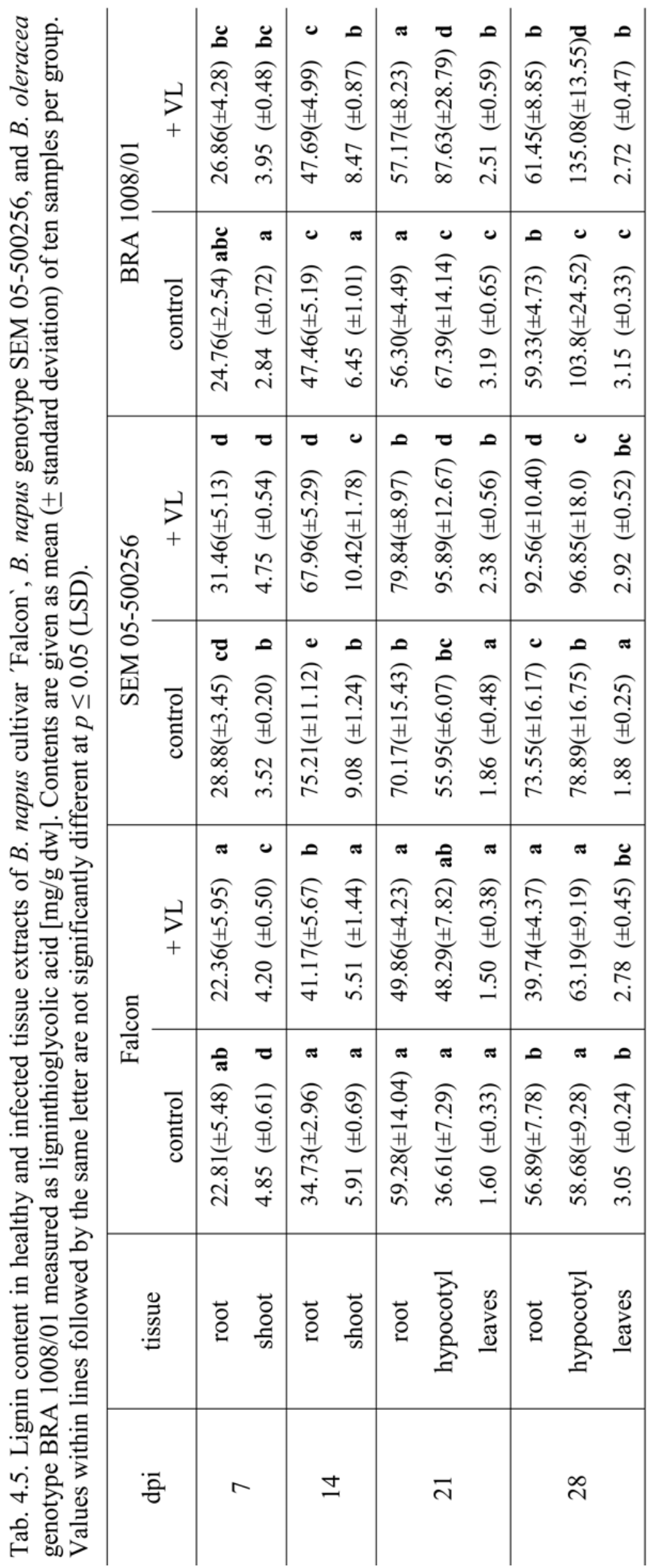


The total content of phenolics, which included soluble phenolic acids, cell wall-bound phenolics and lignin, revealed that the resistant oilseed rape exhibited a higher constitutive levels of phenols in roots and hypocotyls throughout the experiment compared to susceptible plants. However, this was not always true for shoots and leaves. Interestingly, the resistant cabbage always showed significantly higher constitutive as well as induced amounts of total phenols in the upper plant parts, e.g. in shoots (at 7 and 14 dpi), hypocotyls and leaves (at 21 and 28 dpi). In contrast, no clear differences between the resistant genotype and the susceptible oilseed rape was detected in the roots. Changes due to infection, i.e. an increased accumulation of phenolic compounds was most pronounced in the hypocotyl region of resistant SEM 05-500256 and BRA 1008/01 (see Figures 4.9 and 4.10). 

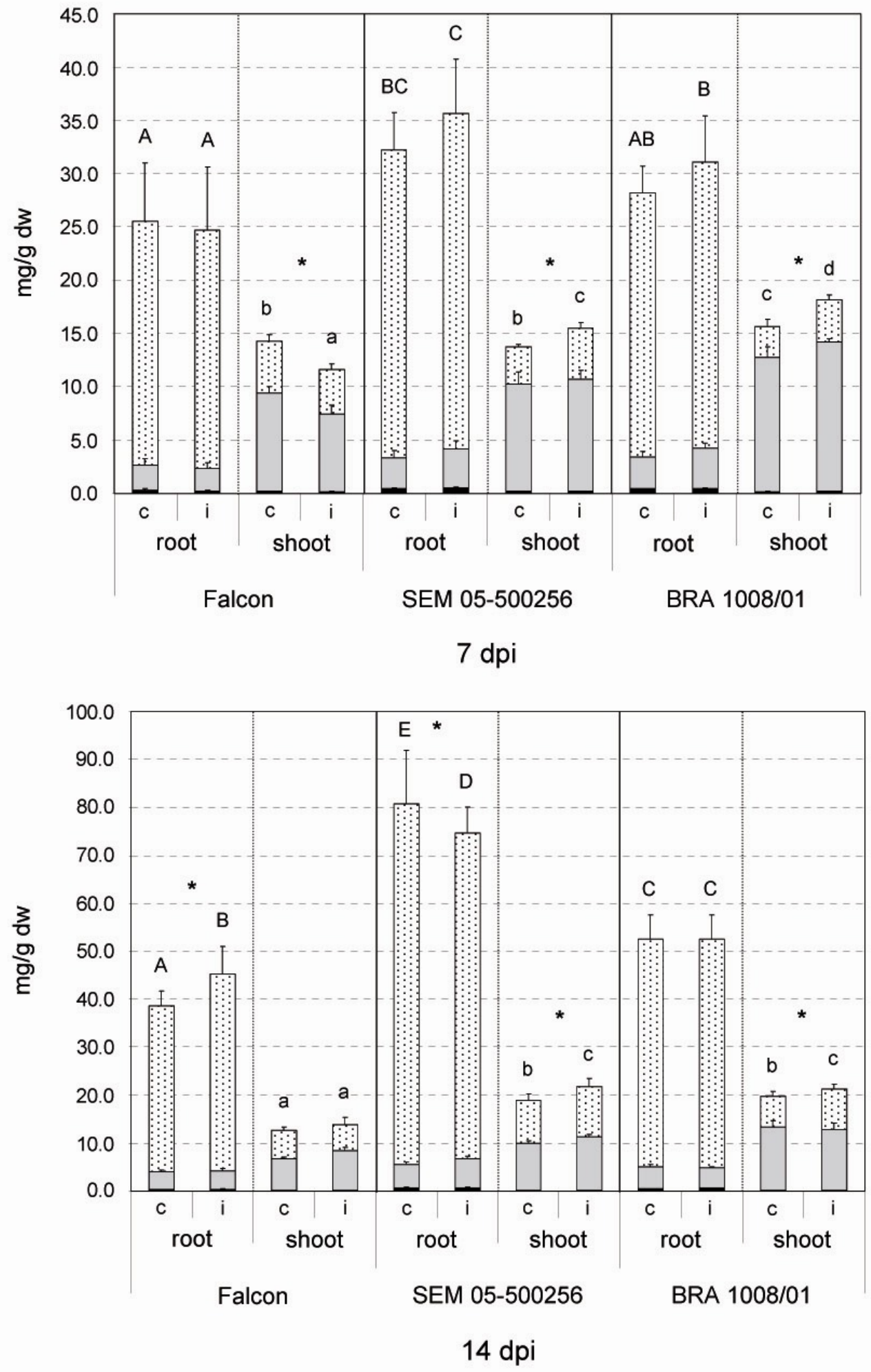

Fig. 4.9. Total phenolic contents, including cell wall-bound phenolic acids (black bars), soluble phenolic acids (grey bars) and lignin (dotted bars) in root and shoot tissue samples of B. napus, cultivar 'Falcon' and genotype SEM 05-500256, and the B. oleracea genotype BRA $1008 / 01$ at 7 and 14 days post inoculation. Content is given in $\mathrm{mg} / \mathrm{g} \mathrm{dw}$ as mean $( \pm$ standard deviation) of 10 samples per group. 

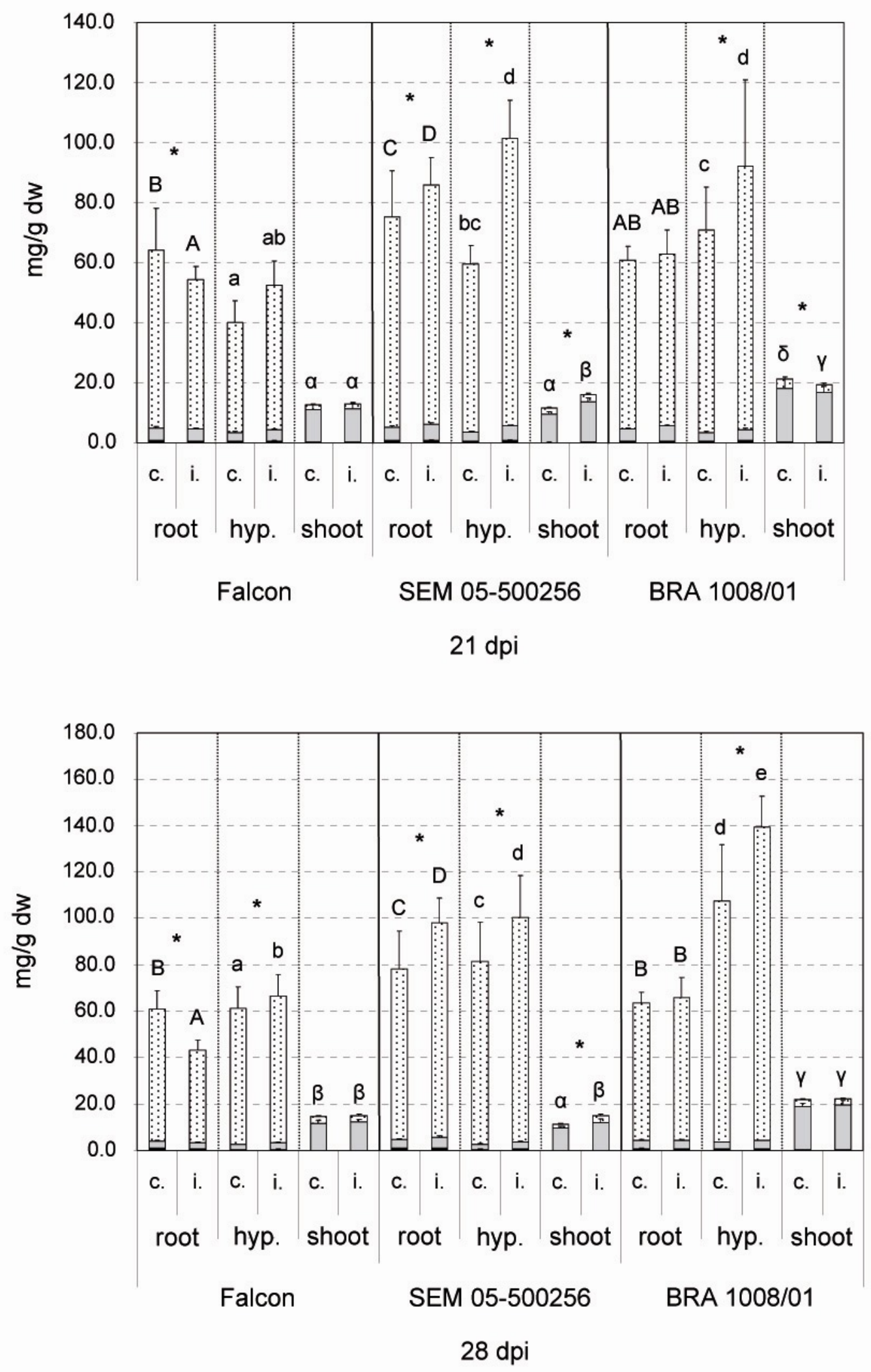

Fig. 4.10. Total phenolic contents, including cell wall-bound phenolic acids (black bars), soluble phenolic acids (grey bars) and lignin (dotted bars) in root and shoot tissue samples of B. napus, cultivar 'Falcon' and SEM 05-500256, and the B. oleracea genotype BRA 1008/01 at 21 and 28 days post inoculation. Content in $\mathrm{mg} / \mathrm{g} \mathrm{dw}$ is shown as mean $( \pm$ standard deviation) of 10 samples per group. 


\section{Discussion}

Successful backfield to infection in vascular diseases depends on the plant's capacity to react with a precise temporal and spatial coordination of induced defence responses during colonization of the vascular tissue (Hammond-Kosack \& Jones, 1996, Kpémoua et al., 1996). When a vascular pathogen, such as Verticillium, has reached the xylem vessels, the progress of the systemic invasion of the plant is facilitated by water flow. At this stage, only xylem parenchyma cells are involved in the defence gene expression which therefore determine the level of plant resistance (Beckman, 1987).

The aim of this work was to characterize resistance mechanisms of $B$. napus to $V$. longisporum and, based on the assumption that resistance had been introgressed from $B$. oleracea to B. napus during interspecific hybridisation (Happstadius et al., 2003; Rygulla et al., 2007a, b), to elucidate whether defence in B. napus and its progenitor species B. oleracea is mediated by similar mechanisms. Emphasis was put on resistance to systemic colonization, including the formation of mechanical barriers and the accumulation kinetics of soluble and wall-bound phenolics as well as the phenolic polymer lignin in a resistant B. oleracea accession and a resistant as well as a susceptible $B$. napus genotype in response to an infection with $V$. longisporum. However, although similar responses were observed in vascular tissues of both susceptible and resistant plants, they occurred with a higher intensity in the resistant varieties. Thus, differences in the response of susceptible and resistant host genotypes were not qualitative but quantitative in nature.

A significant inhibition of fungal spread into upper stem tissues of the resistant oilseed rape variety was revealed by real-time PCR in comparison to susceptible plants. Thus host resistance does not consist in defence to penetration of the root but apparently efficiently restricts the fungus from further spreading upwards into the vascular system of the shoot. The phenomenon of 'internal resistance barriers' restricting fungal growth in less susceptible plants has already been described in other plant-vascular pathogen interactions. In investigations on the cultivar resistance to $V$. dahliae in inoculated cotton plants, the pathogen was isolated less frequently from the apical vascular stem and leaf petiole tissue of the resistant cultivar than from the tolerant or susceptible cotton cultivars. Therefore, it was suggested that factors are involved which inhibit the formation of conidiospores in the xylem (Garas et al., 1986). Studies with Fusarium oxysporum infection of different host plants revealed that resistance is expressed after infection and that roots of both the resistant and susceptible plants are infected (Beckman, 1987). Similarly, in a comprehensive field screening in which numerous cotton lines were tested for resistance to Verticillium wilt, 
Wilhelm et al. (1970, 1974) showed that, although different levels of disease severity were exhibited, none of the tested cultivars were immune to vascular infection. Similarly, Garber and Houston (1966) found no differences in root penetration of resistant and susceptible cotton cultivars by V. albo-atrum. Consequently, success or failure of resistance seems to depend on the rate and extent of defence responses within the vascular system of the host rather than inhibition of root invasion (Beckman, 1990).

A common phenomenon in vascular wilt diseases is the occlusion of vessel elements by gums, gels and tyloses which prevents the spread of the pathogen in the vascular system, a mechanism comprehensively reviewed by Beckman and Talboys (1981) and Talboys (1984). Vander Molen et al. (1977) showed that vascular occlusion by gums and gels can be regarded as a non-specific response of plants to vascular invasion. Beckman and Talboys (1981) hypothesized that the timely occurrence of such blockages serves to limit the entry into the vascular system and thereby functions as a defence mechanism.

The phenomenon of vascular occlusion and particularly the occurrence of vascular gels has been extensely studied in the past and several possible explanations have been offered. For example, they were thought to result from swellings of perforation plates, end walls and side walls of vessels (Beckman \& Zaroogian, 1967) or from the action of pectolytic enzymes on the primary cell walls and middle lamellae (Dimond, 1970; Gothoskar et al., 1955; Deese \& Stahmann, 1962b), therefore explaining their principally pectic composition (Deese \& Stahmann, 1962b). The most recent hypothesis is that in the event of pits being too small to allow the formation of tyloses, synthesis of wall material is induced during pathogenesis, however, and the wall material is extruded to form gels in the vessel lumen. Gel plugs have been described, amongst others, in banana (Beckman et al., 1962; Beckman, 1964; Dimond, 1970), tomato (Scheffer \& Walker, 1953) and carnation (Pennypacker \& Nelson, 1972) and often occur together with tyloses (Pegg \& Brady, 2002).

Globular-shaped tyloses, which result from outgrowths of vessel-associated parenchyma cells through pit cavities into adjacent xylem vessels, often containing nuclei and cell components, are considered to be a common response to vascular pathogens by higher plants (Reichenbach, 1845; Chattaway, 1949), like banana (Beckman \& Halmos, 1962; Beckman et al., 1962), tomato (Chanbers \& Corden, 1963; Dimond, 1970), and sweet potato (Mc Clure, 1950). They contribute to halting the pathogen conveyance within tracheary elements (Beckman \& Talboys, 1981; Vander Molen et al., 1987; Bell, 1992; Ouelette \& Rioux, 1992). Sometimes, tyloses become lignified or suberized, thus becoming resistant to enzymatic degradation (Robb et al., 1979). In a series of investigations, Talboys (1957, 1958a, b) 
showed that there is a negative relationship between the extent of tyloses formation, the degree of colonisation by the pathogen and symptom expression in the interaction Humulus spp./V. dahliae. This is coherent with results from Sinha and Wood (1967a) on tomato plants. Likewise, Tjamos \& Smith (1975) as well as Bishop \& Cooper (1984) attributed resistance of tomato isolines to the formation of tyloses. These findings are in agreement with the results obtained in this study. Here, a positive relation between the abundance of vascular occlusions and the level of resistance was also observed. Furthermore, the blue and red colouration of vascular obstructions in B. napus and the even black staining of vascular occlusions in $B$. oleracea infected with $V$. longisporum after treatment with toluidine blue and phloroglucinol$\mathrm{HCl}$, indicated that these occlusions are impregnated with phenolics and lignin as well as lignin-like polymers.

There are numerous reports on either $V$. dahliae or $V$. albo-atrum providing evidence that mechanisms which result in the occlusion of vessels play a role in plant defence but may also induce water stress, i.e. the expression of wilt symptoms (Bewley, 1922; Threlfall, 1959; Talboys, 1968; Robb et al., 1975a, b). In contrast, the pathosystem Brassica spp./V. longisporum is an example where infection does not result in the typical wilt syndrome. This may have several reasons: First, in contrast to V. dahliae (Jiang et al., 2005; Zhen et al., 2004) and V. albo-atrum (El Assami, 1999), no wilting toxins of $V$. longisporum have been identified so far. Another strategy of infected resistant plants may be to compensate for the loss of vessels by regenerating new xylem elements as has been described in hops and tomato plants after infection with V. albo-atrum (Talboys, 1958b, Pegg \& Selman, 1959). To what extent this compensation mechanism is operative in the interaction B. napus/V. longisporum remains to be elucidated. Finally, even massive inoculation with $V$. longisporum results in a colonisation restricted merely to individual xylem vessels, which might be an explanation for the absence of wilting symptoms in V. longisporum infected oilseed rape, as observed both in the greenhouse and in the field (Eynck et al., 2007).

The phenomenon of vascular occlusion by gels or tyloses cannot be considered without including the involvement of one of the most important compound classes of secondary metabolism as whole and plant defence in particular: the phenylpropanoids. A rapid release of formerly compartmentalized phenolic compounds is thought to be responsible for the host reaction leading to the formation of tyloses and gels that restrict the movement of wilt pathogens within the vascular system (Beckman, 1987, 2000). In this context, so-called phenolic-storing cells play an important role. These are specialized cells that synthesize phenolics and store them in their vacuoles during the normal process of cell differentiation 
(Beckman, 2000). While in most tissues phenolic-storing cells are distributed randomly, in some tissues they appear to be located at strategically important sites where they play a signalling and often a direct role in defence (Wink, 1997). Thus, phenolic-storing cells have been described in xylem tissues of banana, potato, tomato and roots of cotton (Waggoner \& Dimond, 1956; Mace, 1963; Mace et al., 1972; Mace \& Howell, 1974; Müller \& Beckmann, 1974, 1976; Mace et al., 1978). As a response to infection, the phenolic content of these cells is first decompartmentalized and free phenolic acids are secreted into the cell lumen as well as into the extracellular space. There they are immediately oxidized by an enzymatic reaction under consumption of $\mathrm{H}_{2} \mathrm{O}_{2}$. These phenylpropanoid derivatives are involved in the adjustment of the cellular redox status in plants responding to stress (Chong et al., 1999). Furthermore, oxidized phenolic compounds polymerize with each other to form lignin which contributes to sealing off infections at the immediate site of attempted penetration. Finally, if this defence mechanism fails and the stress persists, these same processes promote the prolonged build-up of auxin and ethylene causing a further metabolic cascade which includes secondary metabolism and growth responses. Thus, auxin was shown to promote lateral growth in paravascular contact cells which surround the xylem vessels resulting in the formation of tyloses which, when enlarged, encompass the lumen of vessels (Gordon \& Paleg, 1961; Mace \& Solit, 1966; Matta \& Gentile, 1968; Beckman, 2000).

Mace, (1963) for the first time reported on so-called 'tannin' cells that were randomly distributed in the xylem parenchyma of freshly prepared roots of banana and did not contain polymerized or condensed tannins, but rather a free o-dihydroxyphenol. The observation that phenolics are maintained in a reduced state in the vacuole was confirmed by numerous investigations on phenolic-storing cells of various higher plants (Beckmann \& Müller, 1970; Beckman et al., 1972) and we assume that this is also true for Brassica. This is an important finding because phenolics which are in a free state are normally oxidized and polymerize rapidly. Perpetuation of the reduced state is supposed to be based either on a high $\mathrm{H}^{+}$-gradient between vacuole and cytoplasm (Whetten et al., 1998) or on glucosylation (Dixon \& Paiva, 1995). Following vascular infection of Brassica by V. longisporum, these stored phenolics might be released and diffuse out of their vacuolar compartments into the cell at large. Here, they might become oxidized and polymerize with each other to form polyphenols. These polymerized compounds give a positive staining reaction with toluidine blue as well as FolinCiocalteu reagent, as was observed in the susceptible as well as the resistant interaction. The fact, that resistant SEM 05-500256 and BRA 1008/01 plants exhibited a more distinct accumulation of polyphenols confined to cells immediately adjacent to xylem vessel elements 
all over the cross sectional area of hypocotyls of infected plants, which is distinctly different to the means of accumulation and distribution in the susceptible 'Falcon', leads to the assumption that resistance is at least partly due to a more efficient use of free phenolic compounds. Polymerization with other host constituents, i.e. cellular proteins and cell wall carbohydrates, leads to the formation of "lignified" structures, a phenomenon which was evenly detectable in susceptible and resistant plants infected with $V$. longisporum.

One major effect of soluble phenolic acids, next to their role as regulators of host metabolism, is to directly inhibit microbial growth (Fisch et al, 1973). Thus, protocatechuic acid is known to be responsible for the resistance of pigmented onions to Colletotrichum circinans. Furthermore, chlorogenic acid is supposed to have a critical role in defence responses of plants against fungal pathogens (Yao et al., 1995). It is thought to be involved in the resistance of potato tubers to Phytophthora infestans (Schöber, 1971) and to Streptomyces scabies (Dickinson \& Lucas, 1987). De Ascensao \& Dubery (2000, 2003) reported a substantial increase in total soluble phenolics in FHIA banana roots early after treatment with an elicitor from F. oxysporum f. sp. cubense race 4 . In addition, soluble phenolic compounds have been linked to the resistance of Eucalyptus marginata to P. cinnamomi (Cahill et al., 1993) and of Capsicum annuum to P. capsici (Candela et al., 1995). In this study, the constitutive amounts of soluble phenolic acids present in one B. oleracea and two B. napus cultivars associated with differing resistance to $V$. longisporum showed quantitative differences, as well as the contents in infected tissues. Thus, we provide evidence that there are clear differences in phenolic contents in healthy roots and shoots of the susceptible and the resistant varieties at early time points ( 7 and $14 \mathrm{dpi}$ ), which may contribute to the passive defence of the resistant genotypes. After inoculation, pathogen invasion was associated with marked metabolic changes in soluble phenolics which occurred most strongly in the resistant oilseed rape plants.

Interestingly, changes in phenol concentration in tomato plants infected by different forms of F. oxysporum specific to other hosts were very similar to those occurring as a reaction to race 1 of f. sp. lycopersici in resistant tomato plants (Matta et al., 1969). To which extent this finding can be translated into the incompatible interaction between $B$. napus and $V$. dahliae, can only be speculated.

Phenolic compounds have also been proposed to act as barriers to fungal colonization in pathogenic relationships (Woodward \& Pearce 1988; Kenn, 1992). Thus, the primary reinforcement of the cell wall, which takes place before lignification, is due to the incorporation of hydroxycinnamoyl esters which is initiated immediately on exposure to 
pathogen challenge (Ride, 1983). The ensuing esterification of hydroxycinnamics, such as ferulic acid, to cell wall polysaccharides (Fry, 1982, 1983; Ampomah \& Friend, 1988; Iiyama et al. 1990, 1994) and glyoproteins (Whitmore, 1978a, 1978b) renders these compounds less susceptible to cell wall-degrading enzymes of pathogens. Esters bound to cell wall polysaccharides become cross-linked through the action of extracellular peroxidases and stabilize the cell wall by restricting wall extensibility and digestibility (Fry, 1982, 1987). Furthermore, they are supposed to provide the platform for lignin and suberin biosynthesis (Kolattukudy, 1981; Cottle \& Kolattukudy, 1982; Bolwell et al., 1985; Farmer, 1985; Hahlbrock \& Scheel, 1989; Schmutz et al., 1993; Lam et al., 1992; Bernards \& Lewis, 1992; Matern et al., 1995).

Esterification of phenols to cell-wall materials is a common mechanism in the expression of resistance (Fry, 1987), and the presence of phenols in host cell walls usually increases the resistance to fungal enzymes and represents a physical barrier against fungal penetration. Thus, in the interaction of barley with powdery mildew cell wall-bound phenolics have been reported to contribute to fungal arrest in cell wall appositions (Shiraishi et al., 1989; Zeyen et al., 1995; Lynkjaer et al., 1997) and they have been shown to play a role in the resistance of some wheat cultivars against F. culmorum (Siranidou et al., 2002). The present investigations are in accordance with these findings and show that cell wall-bound phenolic acids are highly likely to contribute to the resistance of Brassica to $V$. longisporum. Thus, similar to the soluble phenolics, the constitutive levels of ester-bound phenolics were substantially higher in the roots of both resistant genotypes, SEM 05-500256 and BRA 1008/01, compared to the roots of susceptible plants, in the first two weeks. At later time points, a pronounced accumulation of cell wall-bound phenolics particularly in the hypocotyls of resistant genotypes was recorded.

Ester-bound phenolics appear to play a role not only in parasitic but also in symbiotic interactions. In investigations on Pinus banksia cells that were treated with elicitors from the ectomycorrhizal fungus Thelephora terrestris accumulated much larger quantities of cell wall-bound phenolic material than the corresponding controls (Campbell \& Ellis, 1992a, b).

Lignin is a three-dimensional phenolic polymer, derived from the free-radical polymerization of the monomeric p-coumaryl, coniferyl and sinapyl alcohols in the plant cell wall, which give rise to p-hydroxyphenyl, guaiacyl and syringyl lignins in various ratios. The relative abundance of these different monolignol residues in lignin varies depending on the plant species (Ride, 1983; Boudet et al., 1995). Vessel walls are supposed to contain only coniferyl alcohol derived guaiacyl lignin (Fergus \& Goring, 1970; Wolter et al., 1974). However, lignin 
synthesized as defence response, may differ in composition, adding an anti-microbial function to the structural one. In normal lignifying cells, lignin is reported to appear first in the apoplastic cellular junctions. Then it spreads between adjacent cells, i.e. in the middle lamella region, and from there through the primary and secondary wall layers (Wardrop, 1971). Accordingly, our histochemical findings of lignin assembly in B. napus and B. oleracea revealed that induced lignification in hypocotyls of infected plants also started in the cell 'corners'.

Besides the fact that a reduction in lignin content has serious physiological consequences on the vascular apparatus (Anterola \& Lewis, 2002) lignin plays a pivotal role in plant defence (Ride, 1978; Nicholson \& Hammerschmidt, 1992). First, lignin increases resistance of walls to mechanical penetration, i.e. compressive forces (Wardrop, 1971; Ride, 1983). Thus, investigations on banana roots infected with $F$. oxysporum revealed that within the context of inducible defence mechanisms cell wall strengthening due to the deposition of lignin plays a major part (De Ascensao \& Dubery, 2000). Secondly, lignification of cell walls at the point of attack may render them resistant to dissolution by fungal enzymes. For example, saccharides are physically shielded from fungal saccharidases by lignin (Friend, 1976). In addition, lignin deposition may restrict diffusion of enzymes and toxins from the fungus to the host, and of water and nutrients from the host to the fungus, eventually starving the fungus. Finally, the hyphal tip of the invading pathogen may become lignified and may lose plasticity required for growth (Ride, 1978).

Numerous reports have emphasised the importance of lignification in plant-pathogeninteractions. Thus, accumulation of lignin and lignin-like polymeric phenolic material has been associated with attempted fungal infection (Bruce \& West, 1989). Friend et al. (1973) reported an increase in lignin $12 \mathrm{~h}$ after inoculation of potato tubers with an incompatible race of $P$. infestans. Accordingly, elicitation of tobacco cells in suspension by elicitor molecules from $P$. nicotianae led to the accumulation of lignin complexes in cell walls $4 \mathrm{~h}$ after elicitation and increased up to ten-fold within 96 h (Oelofse \& Dubery, 1996). Similarly, an elicitor of $P$. sojae was shown to induce a rapid and massive accumulation of phenolic polymers in Glycine max cotyledon cells proximal to the point of elicitor application (Graham \& Graham, 1991). Interestingly, potato tuber tissue that was challenged with a non-pathogen accumulated higher amounts of lignin than tissue inoculated with the pathogen F. roseum $\mathrm{f}$. sp. sambucinum (Hammerschmidt, 1983). It would be worthwhile to elucidate to what extent this also happens in B. napus infected with the non-pathogen $V$. dahliae. From microarray analyses of a tolerant banana cultivar infected with F. oxysporum f. sp. cubense Van den Berg 
et al. (2004) concluded that the disease response involves lignin production and cell wall strengthening due to the incorporation of phenolic compounds into host cell walls. Suppression subtractive hybridization (SSH) revealed gene fragments which showed homology to defence-associated genes, including cell wall-strengthening genes. A significant increase in cell wall-bound esterified phenolics could be observed in this cultivar in response to F. oxysporum f. sp. cubense (Van den Berg, 2007) supporting these results.

Similar to soluble and cell wall-bound phenolics, the biochemical analysis of oilseed rape and cabbage tissue revealed a significant higher constitutive amount of lignin in roots of resistant compared to susceptible plants at early sampling time points. Particularly at 21 days post inoculation, a striking accumulation of lignin was present in the hypocotyls of infected resistant plants, this observation being in good agreement with the histochemical analysis. This finding is supported by results from Smit \& Dubery (1996) demonstrating that cotton hypocotyl tissue responded with increased lignification following treatment with an elicitor from $V$. dahliae. When comparing susceptible and resistant genotypes, the latter exhibited higher levels of lignin-like polymers and induced them earlier. These observations lead to the assumption that the hypocotyl tissue might have a key function in the defence against vascular pathogens.

Considering the total phenolic fraction analysed, resistance of Brassica to $V$. longisporum is differentially expressed according to the time points of the prevailing interaction. Thus, at earlier time points, preformed phenolic compounds play an important role for limiting the extent of infection and colonization by the fungal pathogen. This observation is in agreement with findings where the rate of development of lesions induced by Cercospora nicotianae was considerably higher in plants containing reduced levels of constitutive phenolic compounds as a result of sense suppression of phenylalanine ammonia lyase (PAL) expression (Maher et al., 1994). Similar results were obtained in transgenic potato tubers expressing the Cantharanthus roseus tryptophan decarboxylase gene (Dixon \& Paiva, 1995). In these plants, redirection of tryptophan into tryptamine resulted in decreased phenylalanine pools, corresponding decreases in wound- and elicitor-induced chlorogenic acid and wall-bound phenolics, as well as increased susceptibility to the potato late blight fungus $P$. infestans.

Later stages in the interaction of a resistant Brassica genotype with $V$. longisporum as detected by histochemical and biochemical methods are characterised by a strong accumulation of lignin, lignin-like polymers and other phenolic compounds in the hypocotyls. A similar observation concerning the accumulation of phenolics after infection has been made for stem tissue of Olea europaea infected with V. dahliae (Baidez et al., 2007). Here, the total 
phenol content of the whole stem of infected plants was doubled compared to uninfected plants with oleuropein being the major active compound.

Besides the many similarities between the expression of resistance in $B$. napus and $B$. oleracea found in the present studies, there was also one major difference. While the defence response of $B$. napus was most pronounced in the roots and hypocotyls, $B$. oleracea resistance appears to be expressed primarily in the hypocotyls and leaves. Nonetheless, in both host species defence occurs most pronounced in the hypocotyl tissue and is based on similar mechanisms, suggesting that resistance traits have been introgressed from the B. oleracea into the B. napus gene pool.

\section{Conclusion}

The results obtained in this study are a first step towards understanding the biochemical basis of the genetically conferred resistance of B. napus accessions to $V$. longisporum. Thus, the obstruction of xylem vessels is a conspicuous phenomenon of host response, however, of yet unknown significance. Furthermore, compared to cultivar 'Falcon', the genotype SEM 05500256, similar to the cabbage accession BRA 1008/01, revealed a greater capacity for enhanced phenolic metabolism both with regard to the constitutive and the induced levels of metabolites. Although the present results indicate the involvement of phenolic compounds in defence, their actual role in resistance remains to be analysed in more detail. In conclusion, defence in Brassica to Verticillium can be regarded as a multicomponent dynamic response involving different protective mechanisms which have complementary roles in the overall expression of disease resistance. A better understanding of these mechanisms would contribute to the selection of useful traits for plant breeders attempting to create varieties with improved resistance to $V$. longisporum. 


\section{Chapter 5: General Discussion}

The genus Verticillium contains a number of soilborne, plant pathogenic species with worldwide distribution (Pegg \& Brady, 2002), which have long been known as important plant pathogens. The two most common species, $V$. dahliae and $V$. albo-atrum, are hemibiotrophic fungi causing vascular wilts in a variety of mainly dicotyledonous hosts in temperate and subtropical regions, including hosts such as cotton, cucurbits, alfalfa, sunflower, eggplant, mint, strawberry, tomato and potato (Gordon et al., 2006; Heale, 2000). More recently, a closely related Verticillium species has gained importance representing a distinct taxon consisting of all long-spored isolates with host-adaptation to Brassica species, called V. longisporum (Karapapa et al., 1997, 2000; Steventon et al., 2002a; Fahleson et al., 2003; Fahleson et al., 2004).

Until today, there has been controversy concerning the taxonomic status of $V$. longisporum. Thus, the scientific community is still arguing about whether $V$. longisporum should be regarded as a distinct, host-specific species or not. The proposal for raising $V$. longisporum to species rank was first made by Karapapa et al. (1997) and was supported by many authors who conducted morphological, physiological and molecular analyses (Zeise \& von Tiedemann, 2001; Zeise \& von Tiedemann, 2002a, b; Steventon et al., 2002a, Fahleson et al., 2003; Fahleson et al., 2004). However, reports also exist which suggest that $V$. longisporum should be regarded as a subspecies of $V$. dahliae (e.g. Collins et al., 2003), as originally proposed by Stark (1961), rather than a distinct species. However, just recently, molecular evidence has emerged supporting the status of this fungus as a separate species (Pantou et al., 2006). Besides the controversial taxonomic status, the means of speciation of $V$. longisporum still remains to be clarified. Due to a nuclear DNA-content about 1.8 fold greater than that of V. dahliae isolates, Karapapa et al. (1997) assumed that long-spored Verticillium isolates may have evolved by parasexual hybridization between two different haploid Verticillium species, therefore representing amphihaploids. They suggested that the parental strains concerned both a haploid V. dahliae and a haploid V. albo-atrum isolate. Recent studies (Barbara \& Clewes, 2003; Barbara et al., 2005; Clewes and Barbara, 2005) have shown that V. albo-atrum is most likely not one of the parents but, however, failed to resolve which parental species were actually involved in the parasexual hybridisations that resulted in the emergence of amphihaploid Verticillium isolates. Given the ambiguous state of research, one realizes what challenging task it is 'to taxonomically define a hybrid or hybrid complex in a way that is of practical use' (Brasier et al., 1999). However, as noted by Barbara et al. (2003) it is a 
remarkable coincidence that one of the major hosts of the presumed amphihaploid Verticillium isolate is itself an amphidiploid species, viz. B. napus.

$V$. longisporum, just as V. dahliae and V. albo-atrum, behaves like a classical soilborne pathogen in that its propagules can survive in the soil during extended periods of time in the absence of a host plant (Schnathorst, 1981; Heale \& Karapapa, 1999). After germination and infection of the host roots, the fungus enters the vascular system and subsequently spreads within the sheltering confines of the plant. As the host plant becomes senescent, $V$. longisporum leaves its vascular environment, to which it has been restricted for most of its life cycle and again produces resting structures, so-called microsclerotia, in the dying stem parenchyma and in the roots. Once microsclerotia have returned to the soil, a dormant phase with no saprotrophic growth follows. Therefore, the fungus might be considered as an ecologically obligate pathogen (Barbara \& Clewes, 2003).

\section{Host- and non-host- pathogen interaction studies using confocal laser scanning microscopy}

To our knowledge, this is the first study comparing the infection behaviour of both $V$. longisporum and $V$. dahliae on roots of $B$. napus. The green fluorescent protein was used to label both $V$. longisporum and $V$. dahliae by means of Agrobacterium tumefaciens mediated transformation to visualize their colonization behaviour in the oilseed rape rhizosphere by confocal laser scanning microscopy (CLSM). The GFP methodology was compared with conventional fluorescence staining for its suitability in plant-pathogen interaction studies. Our investigations clearly showed the applied fluorochromes (acid fuchsin, acridine orange) to be superior, because the entire mycelium became visible regardless of its physiologic state. In addition, the faint unspecific staining of the plant tissue enabled a proper localization of the fungus in the host. Nevertheless, labelling plant pathogens with the green fluorescent protein still has several advantages. The method requires no cofactors or substrates, which allows for a fast processing of the plant material, and the risk of potential artefacts is negligible due to the absence of additional chemicals, a feature that enables time-lapse observations in vivo (Lagopodi et al., 2001). Also, GFP tagging is the preferential approach in studies of pathogens under non-sterile conditions.

Novel and significant information about the colonization and infection processes of $V$. dahliae and $V$. longisporum on roots of $B$. napus is provided in this work. This particularly applies to the early interaction, including recognition and first contacts between pathogen and host or non-host. Thus, while $V$. longisporum exhibited a colonization and infection behaviour similar to that observed for other root-infecting vascular pathogens (Lagopodi et al., 2001), the 
interaction of $V$. dahliae with oilseed rape roots entirely differed from $V$. longisporum, indicated by mycelium which is only loosely attached to the root surface and the early and massive production of conidia and microsclerotia outside the root tissue. This observation is in agreement with microscopic investigations from Levy \& Isaac (1976) who were able to show that there is more extensive growth and microsclerotia formation on the root surface of the resistant plant than the susceptible plant which may be regarded as analogous to a host and non-host/pathogen interaction, respectively. Levy \& Isaac suggested that this phenomenon might be related to the failure of the fungus to penetrate the resistant tissue. In our studies, however, both fungi were able to infect epidermal cells, although penetration was observed less frequently for $V$. dahliae. Generally, there was no indication that natural wounds from emerging lateral roots were required for infection, which confirmed previous studies on $F$. oxysporum f. sp. radicis-lycopersici on tomato roots (Lagopodi et al., 2001), F. verticillioides on maize roots (Oren et al., 2002) and V. albo-atrum on roots of cotton (Smith \& Walker, 1930; Garber \& Houston, 1966). In contrast, Reid (1958) suggested that penetration of emerging lateral roots may provide a mechanism for a vascular fungus to avoid the penetration barrier at the endodermis level.

\section{Host and non-host resistance}

Although $V$. dahliae was infrequently able to penetrate and colonize the root tissue, it rarely reached the shoots or leaves as indicated by histological and real-time PCR analyses. The present results demonstrate that the poor susceptibility of $B$. napus to $V$. dahliae is only partly due to the restricted penetration but mainly related to the inhibition of systemic growth, as reported earlier (Zhou et al., 2006). The following questions about the mechanisms governing the expression of this specific type of non-host resistance remain to be answered: Does it embrace inducible defence elicited by the recognition of molecules specific for the particular pathogen, or is it controlled in a non-specific manner by constitutive defence or is defence induced by unspecific stimuli? However, not only non-host resistance but also host-specific resistance expressed in the oilseed rape accession SEM 05-500256 appears to be based on mechanisms preventing the fungus from systemic propagation (Chapter 3, Fig. 3.3; Chapter 4, Fig. 4.1). Thus, while V. longisporum, upon penetration, readily spreads into the vascular system of the susceptible oilseed rape cultivar 'Falcon', systemic growth of the fungus in the resistant genotype was strongly inhibited. These results are in agreement with a number of investigations leading to the assumption that plants often respond in similar ways in host and non-host pathogen interactions, implying the existence of similar defence responses in both 
situations (Heath, 2000; Heath, 2001; Kamoun, 2001; Thordal-Christensen, 2003). However, plants responses greatly depend on the biology of the interactions; therefore it is a difficult task to make useful comparisons between a non-host pathogen interaction and an incompatible host-pathogen interaction. Thus, according to Thordal-Christensen (2003), any statement on the similarity of responses should be based on closely related host and non-host pathogens as given in our investigations.

Some studies dealing with the genetic basis for non-host resistance suggested that non-host resistance that relies on the presence of single genes of the pathogen might be controlled by single plant genes (Fillingham et al., 1992; Wood et al., 1994). Therefore, one may assume that non-host resistance includes similar gene-for-gene interactions than pathogen-specific resistance within a host species. As a matter of fact, there is molecular evidence of gene-forgene interactions controlling non-host resistance of some cereals to incongruous races of powdery mildew fungi (Matsumura \& Tosa, 1995). However, based on the assumption that host resistance of $B$. napus to $V$. longisporum is mediated by several resistance loci (Happstadius et al., 2003) it is assumed that non-host resistance of oilseed rape to V. dahliae relies on the expression of several genes. According to Heath $(1985,1997)$ non-host resistance to fungi is generally assumed to be under complex genetic control and therefore may involve a multitude of defence mechanisms. Thus, polygenic non-host resistance might be explained by the way that several plant resistance gene alleles respond to the products of several corresponding avirulence gene alleles in the pathogen (Heath, 2001). Furthermore, it is assumed that non-host resistance genes, like genes that code for host-specific resistance, may be distributed differentially among plant genotypes within the non-host species (Fillingham et al., 1992; Wood et al., 1994). Finally, the fact that the non-host pathogen $V$. dahliae is able to penetrate root epidermal cells and colonize the root and stem tissue of oilseed rape to a certain, although limited extent, might indicate that this sort of non-host resistance is governed by genes directly involved in defence rather than pathogen recognition.

\section{Sites of resistance expression}

The most common type of expression of host resistance, also frequently found with non-host resistance, is the hypersensitive response (HR), which comprises a rapid cell death immediately at the site of infection. This phenomenon is associated with the limitation of the pathogen as well as with the activation of defence-related genes (Goodman \& Novacky, 1994). However, in this study no indication for the elicitation of hypersensitivity in affected tissue was detectable, neither during root infection by the non-host pathogen $V$. dahliae nor 
during the early stages of interaction between $V$. longisporum and a resistant $B$. napus genotype. According to Pegg \& Brady (2002), who compiled a comprehensive review on the vast literature on vascular wilts, there are generally no reports on a hypersensitive response (HR) in roots to vascular pathogens. Furthermore, complete resistance to infection by this class of pathogens has never been proven. Thus host plants with monogenic and polygenic resistance identified so far, consistently show at least limited root or hypocotyl colonization. In general, HR has seldomly been described as a defence response to root-infecting fungi. Examples are the pathosystems Arabidopsis thaliana/Plasmodiophora brassicae (Fuchs \& Sacristan, 1996), Gycine max/Phytophthora sojae (Kosslak et al., 1996) and Linum usitatissimum/Fusarium oxysporum (Olivain et al., 2003). However, the HR described in soybean can be regarded as an exception because it resulted from a recessive mutation and at least for the $P$. brassicae-Arabidopsis pathosystem the occurrence of HR in the roots was questioned (Hermanns et al., 2003), because cell death was clearly associated with lignification. Due to the fact that the microscopic investigations by Fuchs \& Sacristan were performed fairly late after infection, the authors thus failed to prove that cell death was really a consequence of an HR in the sense of programmed cell death rather than a long-term effect of lignification. The majority of root-infecting fungi are necrotrophs like Pythium, or hemibiotrophs like Phytophthora, and resistance to these categories of pathogens is generally not associated with an HR (Klement, 1986). Additionally, non-host resistance to fungi often does not involve cell death but inhibition within intercellular spaces or growth restriction within cell walls (Heath, 2000). A possible reason for this might be the fact that strong defence responses such as HR-mediated cell death in the roots would endanger the whole plant. This appears particularly relevant as the roots are associated with a strongly enriched microflora. Thus, if roots would initiate a HR when confronted with any possibly deleterious microorganisms, they would die and thus endanger the existence of the whole plant (Hermanns et al., 2003).

Given these considerations, it appears reasonable that primary resistance determinants in the roots are principally depending on mechanisms other than HR (Mansfield, 2000), such as the formation of so-called lignitubers which prevent further colonization of the roots by the respective fungi. Those have been reported by several authors (Van der Meer, 1925; Talboys 1958; Presley et al., 1966; Griffiths, 1971; Bishop \& Cooper, 1983a). However, much uncertainty has been associated with the role and chemical composition of these structural barriers and thus their role in resistance is still considered as ambiguous (Wood, 1967; Bishop \& Cooper, 1983a). Accordingly, in our microscopic studies, we could not detect any sort of 
cell wall appositions as a response of the plant to infection in either host- or non-hostpathogen interaction. The Casparian strip, a suberized part of the endodermis surrounding the central cylinder, is generally thought to act as a natural physical barrier against Verticillium infection in roots (Talboys, 1958). This obstacle can be easily overcome either when damaged or through longitudinal growth from the elongation zone, where the vascular system is not yet fully developed and the endodermis has not yet differentiated (Pegg, 1974; Schnathorst, 1981; Huisman, 1982; Bowers et al., 1996).

The lack of an effective defence response during the early stages of infection suggests that the initiation of resistance mechanisms might be displaced from the infection site to more distal tissue parts where strong defence responses including cell death are less harmful for the plant. This relocation phenomenon has been described for sunflower infected with the biotrophic oomycete Plasmopara halstedii, the causal agent of downy mildew (Mouzeyar et al., 1993; Radwan et al., 2005). Although resistance of sunflower is associated with HR in the roots, it fails to halt the parasite resulting in the colonization of both susceptible and resistant cultivars. However, the HR triggers a systemic disease response in the sense of a systemic aquired resistance (SAR) which takes place in the upper part of the hypocotyls and leads to the arrest of the pathogen. This general phenomenon in sunflower after infection with downy mildew is called Cotyledon Limited Infection (CLI, Gulya et al., 1991). Accordingly, in our system, both in the host and non-host interaction, defence mechanisms become operative in the vascular system of the plant resulting in the impairment of the acropetal spread of the pathogen, as indicated by means of real-time PCR analyses. Principally, the localization of vascular infections is widely recognized as a primary resistance mechanism to wilt pathogens, meaning that fungal dissemination is limited in resistant cultivars, while extensive colonization occurs in susceptible plants. In the genus Fusarium it has been reported that symptom expression is determined by the extent of colonization (Elgersma et al., 1972; Conway \& Mc Hardy, 1978). With regard to this, Verticillium is exceptional as there is little or no correlation between fungal proliferation and symptom development (Schnathorst, 1981; Brandt et al., 1984; Corsini et al., 1988; Gold et al., 1996; Lynch et al., 1997; Heinz et al., 1998; own investigations, data not shown), although the ability of the pathogen to rapidly colonize the host might play a role in disease development and severity (Gold et al., 1996). However, we obtained a clear negative correlation between the level of resistance of the host plant and the extent of fungal colonization of the stem. For tomato infected with $F$. oxysporum f. sp. lycopersici the slope of the regression line fitted between the height reached by the fungus up in the stem and the time after inoculation provided a measure of resistance in 
different cultivars (Rodriguez-Molina et al., 2003). Other authors, using the same pathosystem, calculated a colonization ratio to obtain a quantitative expression of the rate at which the vascular system of plant stems had been colonized, which in turn served as a parameter of disease resistance (Gao et al., 1995a, b). The localization of infections within basal vascular tissues as a primary defence mechanism has been reported in several previous reports. A short review is given in the introductory part of this thesis.

\section{Potential resistance factors}

Considering these observations and given the challenge to identify potential resistance factors in the host-pathogen interaction, we placed emphasis on defence reactions occurring in the plant's vascular system during the incompatible interaction between $V$. longisporum and a resistant B. napus genotype. These reactions included the formation of mechanical barriers and the accumulation kinetics of soluble and wall-bound phenolics as well as the phenolic polymer lignin. Additionally, this study provides evidence for the assumption that resistance in B. napus originates from B. oleracea and has probably been introgressed through interspecific hybridisations between the ancestral species B. oleracea (cabbage) and B. rapa (turnip rape).

Microscopic observations clearly revealed the occurrence of structural alterations in both susceptible and resistant plants in response to infection with $V$. longisporum. In addition to the occurrence of vascular obstructions, non-specific wall-associated defence responses were triggered in resistant as well as susceptible plants. Although similar responses, viz. changes in the redox state and assembly of soluble phenolics, reinforcement of constitutive barriers and formation of vessel occlusions occurred in vascular tissues, they were observed with a higher intensity in the resistant accession of B. napus and B. oleracea. Thus, differences in responses between the susceptible and the resistant interaction seem to be quantitative rather than qualitative. However, intensities of responses were difficult to quantify, which is a general disadvantage of approaches based on microscopic imaging. Furthermore, histochemical analyses do not differentiate individual classes of compounds. In contrast, biochemical analyses revealed significant differences with regard to the constitutive quantities of the prevailing classes of phenolics (soluble or cell wall-bound phenolic acids, and lignin) in control plants of genotypes exhibiting different levels of resistance and with respect to events induced after infection.

Similar to several previous studies on hops and tomato (Talboys, 1957, 1958a, b; Sinha and Wood, 1967a; Tjamos \& Smith, 1975; Bishop \& Cooper, 1984), our investigations indicated a 
positive correlation between the occurrence of vascular occlusions and the level of resistance. Vascular gels and tyloses belong to those defence mechanisms against fungal vascular pathogens that have been considered most frequently to contribute to pathogen confinement in the vascular system (e.g. Talboys, 1958; Sinha \& Wood, 1968; Tjamos \& Smith, 1974, 1975; Beckman, 1990). Based on the assumption, that vascular occlusions represent a non-specific response of plants to vascular invasion (Vander Molen et al., 1977), Beckman \& Talboys (1981) hypothesized that the timely occurrence of blockages serves as a defence mechanism. However, our histological data reflect the situation at one definite time point and do not depict a time-course development. Due to this, the question of to what extent the timely occurrence of occlusions, besides their quantitative incidence, might play a role in the interaction Brassica / Verticillium remains to be answered.

Though we did not elucidate whether gels or tyloses caused the obstruction of vessels, however, the blue and red colouration of these vascular obstructions in B. napus and the black staining of vascular occlusions in B. oleracea after treatment with specific dyes indicates that oxidized phenols diffused from xylem parenchyma cells into the gel plugs or tyloses in the vessels, rendering them less susceptible to degradation by fungal enzymes. The capacity of phenolics to infuse and stabilize wall structures has been verified in vitro and in vivo (Beckman et al., 1974). However, phenolic compounds do not only serve as soaking substances. In fact, some phenolic compounds are of great significance in constitutive plant resistance while others are synthesized after elicitation (Mansfield, 1983) such as the flavonoid and terpenoid phytoalexins of cotton (Joost et al., 1995). Phytoalexins that play a role in the defence of Brassica spp. against fungal pathogens are for example brassinin, spirobrassinin, cyclobrassinin, brassilexin, rapalexin or rutalexin (Pedras et al., 2007). All theses compounds are not of phenolic origin; furthermore the existence of any phenolic phytoalexin has not been described for Brassica species so far.

In general, phenolic compounds play an important role in stress physiology, and it is likely that their major effect is not direct inhibition of microbial growth but regulation of host metabolism in disease resistance. Thus, formerly decompartmentalized phenolics that are released by so-called phenolic-storing cells (Beckman, 1987, 2000) are thought to be responsible for the host reaction leading to the formation of tyloses and gels, under consumption of $\mathrm{H}_{2} \mathrm{O}_{2}$ (Chong et al., 1999) and the action of auxin and ethylene (Gordon \& Paleg, 1961; Mace \& Solit, 1966; Matta \& Gentile, 1968; see chapter 4). Furthermore, oxidized phenolic compounds polymerize with each other to form lignin which contributes to sealing off infections at the immediate site of attempted penetration (Nicholson \& 
Hammerschmidt, 1992). Cell wall reinforcement due to phenolic infusion has also been described in the interaction of several plants with different formae speciales of Fusarium oxysporum (Baayen et al., 1989; Benhamou \& Garand, 2001; Rodriguez-Galvez \& Mendgen, 1995; Salerno et al., 2000). In our studies, genotype SEM 05-500256 and cabbage accession BRA 1008/01 revealed a greater capacity for phenolic metabolism with regard to the basal as well as the induced levels of metabolites in comparison with cultivar 'Falcon'.

The basic mode of resistance expression of both Brassica genotypes to $V$. longisporum turned out to change during the course of the interaction. Thus, at earlier time points, analyses revealed an important role for preformed phenolic compounds in limiting infection and colonization by the fungal pathogen, especially in the roots. Later stages are characterised by a strong accumulation of lignin, lignin-like polymers and other phenolic compounds in the hypocotyls. This finding is in agreement with results from Smit \& Dubery (1996) who demonstrated that cotton hypocotyls responded with an increased lignification after treatment with an elicitor from $V$. dahliae whereby the resistant genotype exhibited higher levels of lignin-like polymers and induced them earlier than the susceptible one. These observations lead to the assumption that the hypocotyl has a key function in the blocking of vascular pathogens.

The browning of vascular elements and adjacent tissue, indicative of the oxidation of free phenolic compounds released after infection, is a frequently observed phenomenon of wilt diseases similar to the presence of mycelium in the vessels (Pegg \& Brady, 2002). Accordingly, this macroscopically visible phenomenon was also observed after infection with $V$. longisporum, being most pronounced in the susceptible oilseed rape cultivar. To date, there is still ambiguity as to whether an increased level of phenolics after infection might be regarded as a consequence of disease expression or as part of the plant's defence apparatus and a distinction between these two phenomenons is diffcult to draw. However, one has to keep in mind that host metabolites might have multiple, as well as interacting functions that might have an effect on symptom development (Pegg \& Brady, 2002). Davis \& Dimond (1954), Davis et al. (1953) and Waggoner \& Dimond (1956) presented data in support of the hypothesis that free phenols responsible for vascular browning in tomato stems are derived from conjugated phenols which undergo hydrolysis and enzymatic oxidation in the diseased plant. Same was reported for banana infected with F. oxysporum f. sp. cubense (Mace \& Wilson, 1964). The constant association between initial vascular browning and the appearance of histochemically reactive phenols in scattered xylem parenchyma cells of the stem axis indicates that the substrates for early vascular browning are localized in these cells. The 
failure to detect histochemically reactive phenols in the xylem parenchyma of healthy stems implies, as already suggested by Davis et al (1953) and Waggoner \& Dimond (1956) that phenols may occur in conjugated forms in healthy tissue and are released as free, reactive phenols only after enzymatic hydrolysis in the diseased plants. In this context, the oxidation of phenols to brown products diverts the supply of precursors for lignin biosynthesis. This assumption is supported by the results of our biochemical analysis, showing a decrease of the total content of free phenolics in roots of the susceptible genotype. In turn, the accumulation of oxidized phenolic compounds might have resulted in the brown discoloration of xylem vessels and adjacent parenchyma tissue in both the roots and hypocotyls. We observed an inverse correlation between the frequency of tyloses and the occurrence of vascular browning in Brassica plants with different levels of resistance when infected with V. longisporum. According to Beckman (1966), phenolic infusion responsible for vascular browning can prevent the normal plasticizing of walls and inhibit the growth of tyloses at infection sites. It seems that a sensitive balance and timing must be maintained between the mediation of auxin by phenolics, and the infusion of phenolics into wall structures, if the defensive system is to be effective.

During this work, many similarities in the expression of resistance in $B$. napus and $B$. oleracea have been observed. However, there was one major difference. While the defence response of $B$. napus was most pronounced in the roots and hypocotyls, resistance in $B$. oleracea appeared to be expressed primarily in the hypocotyls. In both species defence occurs most strikingly in the hypocotyl tissue and is based on the same mechanisms. This supports the hypothesis that resistance traits in B. napus have been introgressed from B. oleracea. 


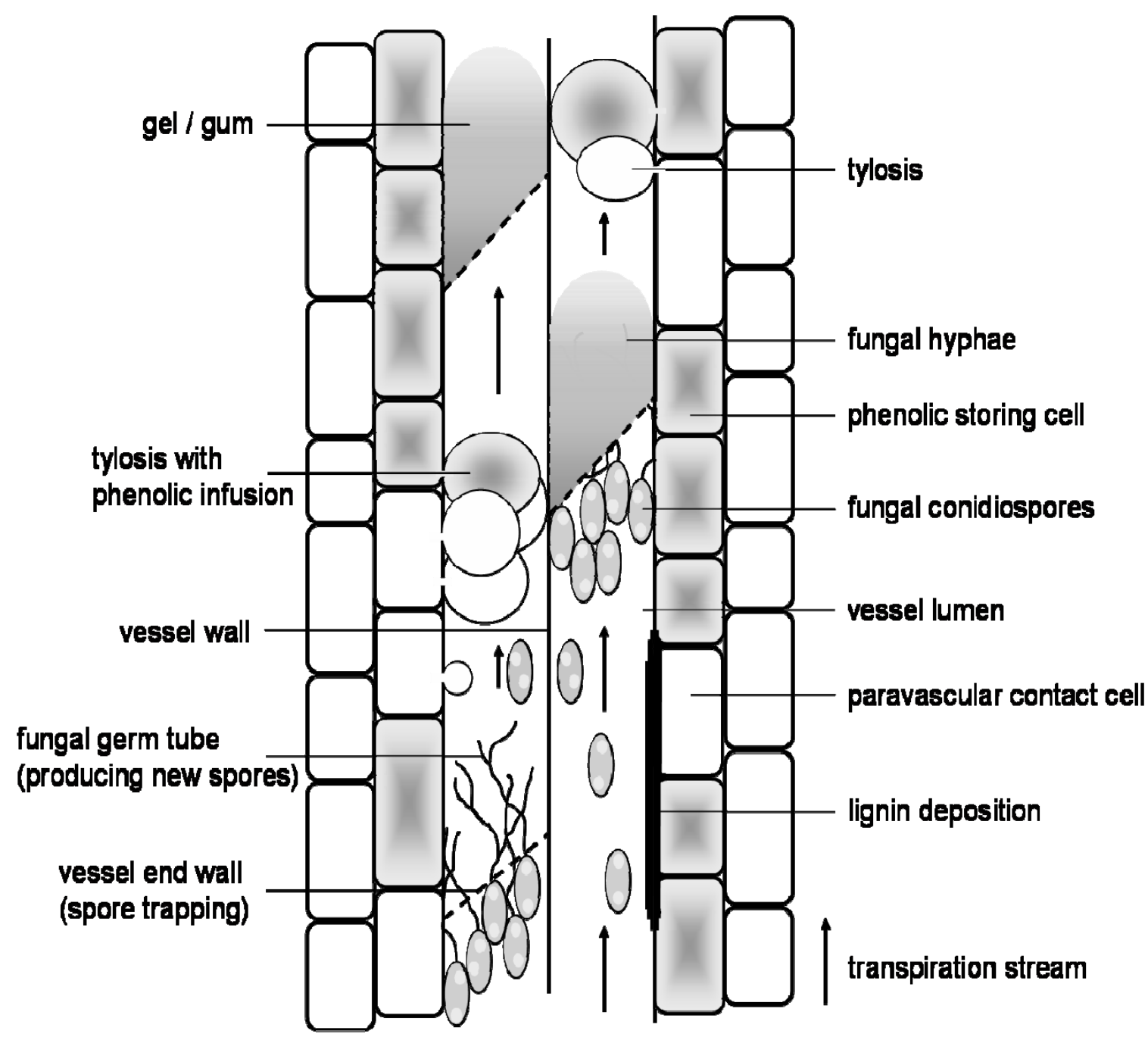

Fig. 5.1. Model of longitudinal and lateral acting host resistance factors within the vessel elements and the surrounding vascular parenchyma tissue in the interaction between Brassica spp. and $V$. longisporum. The scheme is based on histochemical as well as biochemical data obtained during this work.

\section{Resistance in Brassica napus}

B. napus is a spontaneous interspecific hybrid between turnip rape (B. rapa syn. campestris, genome AA) and cabbage (B. oleracea, genome $\mathrm{CC}$ ) and therefore is an amphidiploid species (genome AACC). Intensive breeding for oil and seed quality has resulted in a fairly narrow genetic basis and potentially a relatively low level of resistance to V. longisporum. However, some promising progress in resistance breeding has recently been reported (Happstadius et al., 2003) indicating that the related species B. oleracea represents a valuable source of resistance. In a comprehensive greenhouse screening, the existence of high resistance levels in several cabbage gene bank accessions was confirmed. In order to enlarge the gene pool available for resistance breeding, some of the interesting genotypes were used in interspecific crossings with B. rapa with the long-term goal of improving the level of resistance to $V$. longisporum in 
B. napus (Rygulla et al., 2007a, b). Similar to the results of Happstadius et al. (2003), resynthesized oilseed rape lines with a resistance level considerably higher than the tolerant winter oilseed rape cultivar 'Express' were obtained. More detailed information about the disease response of the parental accessions and the achieved level of resistance in the newly generated oilseed rape genotypes is contained in the paper by Rygulla et al. (2007a, b).

In the past, the strategy of resynthesizing novel B. napus genotypes from the two diploid progenitor species has been applied for the introduction of resistance genes against turnip yellows virus (TuYV, Dreyer et al., 2001), turnip mosaic virus (TMV, Walsh et al., 1999) and also fungal pathogens, such as Leptosphaeria maculans (Crouch et al., 1994) and Plasmodiophora brassicae (Diederichsen \& Sacristan, 1996). In all these studies, the introduced resistance alleles originated from the B. rapa parent. However, the use of a specific resistance from only a single donor source in some cases might exert a strong selection pressure on the pathogen that can result in the resistance being overcome rapidly, as has recently been shown for the resistance against Plasmodiophora (Fähling et al., 2003) and Phoma (Sprague et al., 2006). Thus, the aim of the study by Rygulla et al. (2007a, b) was to identify new resistance sources against $V$. longisporum in $B$. rapa gene bank material, and to combine these with known B. oleracea resistance sources in novel resynthesized B. napus lines in order to generate long-term resistance and to avoid the formation of new pathotypes with enhanced aggressiveness. By combining different $\mathrm{A}$ and $\mathrm{C}$ genome resistance sources in new resynthesized $B$. napus lines, interesting new breeding material has been generated for the introgression of genetic variation for quantitative resistance to $V$. longisporum into elite oilseed rape cultivars.

\section{Verticillium longisporum as a model for studying vascular fungal pathogens}

In order to address the key issues in fungal pathogenesis, model systems allowing easy experimental manipulation and a rapid testing of hypotheses are required (Roncero et al., 2003). For airborne plant pathogens, a number of models exist, most prominently Magnaporthe grisea (now: M. oryzae; Hamer \& Talbot, 1998) and Ustilago maydis (Banuett, 1995). No such model exists for soilborne fungal pathogens or particularly vascular fungal pathogens. This is hardly understandable given (i) the unique mode of parasitism by Verticillium and other species colonizing the xylem, (ii) the growing importance particularly in extensive oilseed rape cropping areas, (iii) the easy in vitro handling, (iv) established genetic transformation techniques for both Verticillium dahliae and $V$. longisporum (Dobinson, 1994; Eynck et al., 2007), (v) the abundance of biological, physiological and 
biochemical data on pathogenesis of $V$. dahliae and $V$. albo-atrum, (vi) the existence of a major gene for resistance to Verticillium in tomato, cotton, sunflower and potato, and finally (vii) the existence of Arabidopsis thaliana as a closely related model host plant for Verticillium (Cooper, 1999). Thus, Verticillium holds considerable potential as an experimental system to advance the knowledge of fungal infection, both for aspects of general interest and for those specific for vascular pathogens. However, there are some constraints that hamper the molecular handling of the pathogen. Thus, tests for the contribution of particular genes to pathogenicity are thwarted by the amphihaploidy of the fungus, which is due to the fact that $V$. longisporum comprises two nearly complete sets of haploid genomes in one single nucleus. Therefore the production of knock-out mutants completely lacking a particular feature or activity through the inactivation of the responsible gene represents an extraordinary challenge. However, the function of genes can be elucidated through the generation of knock-down mutants based on sequence-specific gene silencing via transgenic DNA or various RNAi techniques (dsRNA interference) which result in preventing the translation of a particular mRNA to the corresponding protein. Since sequence data at least from $V$. dahliae will be available soon (NSF/USDA Microbial Genome Sequencing Program FY 2006) and functional genomic tools are being established (Pantou et al., 2006; Tappe et al., unpublished; Weiberg et al., unpublished), Verticillium may become an invaluable model for studying pathogenesis of vascular fungal plant pathogens.

\section{Conclusions}

Presently, growers have limited options to control Verticillium wilt on oilseed rape due to the longterm survival of inoculum in the soil and the systemic growth of the pathogen which renders any attempts of chemical or cultural control ineffective. Thus, planting resistant varieties has the potential of becoming a unique and prime strategy in the management of this root pathogen. Given these considerations, the results obtained in this work are a first step towards understanding the basis of the genetically conferred resistance of $B$. napus to $V$. longisporum. Although our data indicate the involvement of phenolic compounds, particularly lignin, in defence, their actual role in resistance needs to be subjected to further ensuing studies. In conclusion, defence in Brassica to Verticillium can be regarded as a dynamic response comprising a multiplicity of mechanisms acting in a distinct time course pattern and having complementary roles in the overall expression of disease resistance. The combination of greenhouse and field resistance trials with further investigations regarding the molecular and biochemical mechanisms which underlie defence may contribute to a better understanding 
of resistance to Verticillium and thus strengthen the efforts in breeding varieties with improved resistance to $V$. longisporum. 


\section{Summary}

The increasing area of oilseed rape cultivation particularly in the northern European countries has significantly promoted Verticillium longisporum, the causal agent of 'Verticillium wilt' of Brassica crops, and thus has rendered this disease a major challenge for current resistance breeding efforts.

V. longisporum (ex. V. dahliae var. longisporum Stark; comb. nov. Karapapa et al.) is a soilborne fungus which exhibits a life cycle similar to other plant pathogenic Verticillium species, like $V$. dahliae and V. albo-atrum. Infection is initiated by hyphae from germinating microsclerotia which invade the plant vascular system through penetration of the fine roots. Most of its life cycle, $V$. longisporum is confined to the vascular system of the plant. In oilseed rape, no typical wilt symptoms are induced but a reduction in growth and premature senescence are observed which may severely reduce yields. Microsclerotia produced in infected plant tissue are released into the soil after rapeseed harvest and represent a durable contamination in the soil for several years. Furthermore, V. longisporum can not be controlled with fungicides. The most promising means of controlling Verticillium within the scope of an integrated pest management in addition to the implementation of cultural practices is the use of resistant cultivars. Until now, for both winter and spring type oilseed rape, breeding for resistance has been severely hampered by the absence of sufficient resistance in commercially available breeding material. However, recently, some promising genotypes of cabbage ( $B$. oleracea) with enhanced resistance were identified. With these considerations, the chief objectives of this dissertation were to identify new sources of resistance and to characterize resistance factors in oilseed rape genotypes as well as in related Brassica forms with the longterm goal of improving the resistance of winter oilseed rape against $V$. longisporum.

One major part of this work is concerned with the screening of different Brassica species, including B. napus, resynthesized oilseed rape forms and the progenitor species B. oleracea and B. rapa (syn. campestris) both in greenhouse and field studies. Our results confirm the elevated level of resistance in B. oleracea genotypes. Furthermore, it is demonstrated that the tested B. rapa assortment primarily exhibited a low to moderate resistance phenotype whereas accessions of B. napus on the average took a medial position. Due to the lack of correlation between greenhouse and field results, it is concluded that the eligibility of the screening assay as an isolated test method is equivocal. Thus, in order to identify genotypes that are undoubtedly resistant not only under standardized but also under field conditions, a 
combination of upstream greenhouse screenings and ensuing resistance tests in the field at several locations is indispensable.

A further objective of this work was to identify mechanisms contributing to the overall resistance phenotype of Brassica varieties to $V$. longisporum and potentially useful for oilseed rape breeding programmes. In principle, two different approaches were pursued throughout this project: In the first instance, potential levels of resistance were identified by investigating the interaction of $B$. napus with a virulent, host-specific isolate of $V$. longisporum in comparison to the interaction with a host-heterologous strain of $V$. dahliae.

In the first approach, the differential interactions of $V$. longisporum and $V$. dahliae on the root surface and in the root and shoot vascular system of B. napus were studied by confocal laser scanning microscopy (CLSM), using GFP tagging and conventional fluorescence dyes. The results of these studies revealed a superior performance of the fluorescence dyes in comparison to the use of GFP. Furthermore, this study provided novel information about the early stages of infection and colonization in the host and non-host-pathogen-interaction. Although $V$. dahliae was infrequently able to penetrate roots of $B$. napus, it failed to spread further into the shoot, as shown with real-time PCR. This kind of restricted invasion of a vascular pathogen implies a systemic non-host resistance, the mechanisms governing its expression being not known.

Furthermore, screening material with different levels of resistance to $V$. longisporum, including a susceptible and a resistant $B$. napus genotype and a resistant B. oleracea variety, was used to differentially characterize relevant resistance factors. Based on the results obtained from the non-host interaction, emphasis was placed on resistance to systemic colonization. As a result, mechanisms were found which become operative after the pathogen has entered the plant, such as the build up of mechanical barriers like tyloses or vascular gels, as well as the reinforcement of constitutive barriers through the deposition of cell wall-bound phenols and lignin. Furthermore, the accumulation of soluble phenolics potentially acting as inhibitors of microbial growth or also as regulators of host metabolism in disease resistance was observed. Although similar responses occurred in vascular tissues of both resistant and susceptible plants, they occurred with a higher intensity in the resistant B. napus and $B$. oleracea accessions. Thus, from these studies, differences in responses between the susceptible and the resistant interaction can be regarded as being quantitative rather than qualitative in nature. In both resistant Brassica genotypes the most active defence occurs in the hypocotyl tissue and seems to be based on similar if not the same mechanisms. This 
clearly corroborates the hypothesis that resistance traits in B. napus have been introgressed from B. oleracea.

The results obtained from this study are a first step towards understanding the biochemical basis of the genetically conferred resistance of $B$. napus accessions to V. longisporum. Future research should aim at further elucidating the underlying mechanisms of host as well as nonhost resistance. Thus, an integrated approach combining issues of plant pathology and plant breeding could contribute to the selection of traits which might be useful for plant breeders attempting to generate varieties with improved resistance to $V$. longisporum. 


\section{Zusammenfassung}

Die zunehmende Anbaufläche von Raps hat insbesondere in Ländern Nordeuropas zu einem verstärkten Auftreten von Verticillium longisporum, dem Erreger der sogenannten Verticillium-Welke an Brassica- Arten, geführt.

V. longisporum (ex. V. dahliae var. longisporum comb. nov. Karapapa et al.) ist, wie V. dahliae und $V$. albo-atrum, ein bodenbürtiger Pilz mit einem monozyklischen Infektionszyklus. Ausgehend von keimenden Mikrosklerotien werden vornehmlich Feinwurzeln infiziert. Den größten Teil des Lebenszyklus ist $V$. longisporum auf das vaskuläre System der Pflanzen beschränkt. Im Gegensatz zu anderen Verticillium- Arten verursacht $V$. longisporum an Raps keine Welkesymptome, statt dessen treten Stauche und verfrühte Abreife auf, aufgrund derer die Bezeichnung der Krankheit als „VerticilliumWelke“ irreführend ist und eine Umbenennung in Betracht gezogen werden sollte. Mikrosklerotien, die in infiziertem Pflanzengewebe gebildet werden, gelangen nach der Ernte in den Boden und verseuchen diesen auf Jahre hinaus. Des Weiteren kann der Pilz nicht mit Hilfe von Fungiziden bekämpft werden. Ein alternativer Ansatz zur Bekämpfung von $V$. longisporum im Rahmen eines integrierten Pflanzenschutzes ist der Anbau resistenter Sorten. Bislang wurde die Resistenzzüchtung sowohl im Winter- als auch Sommerraps erheblich durch das Fehlen einer ausreichenden Toleranz bzw. Resistenz im vorhandenen Zuchtmaterial erschwert, wenn auch kürzlich einige vielversprechende Kohl-Genotypen mit einem erhöhten Resistenzniveau identifiziert wurden. Vor diesem Hintergrund war es das Ziel dieser Arbeit, neue Resistenzquellen $\mathrm{zu}$ identifizieren und mögliche Resistenzfaktoren in Raps und verwandten Brassica- Formen zu charakterisieren, um die Resistenz von Winterraps gegenüber $V$. longisporum langfristig zu verbessern.

Verschiedene Brassica-Arten, darunter B. oleracea- und B. rapa- (syn. campestris) Varietäten sowie Raps- und resynthetisierte Genotypen, wurden im Gewächshaus wie auch unter Feldbedingungen hinsichtlich ihrer Resistenzeigenschaften getestet. So sollten resistente oder zumindest teilresistente Genotypen identifiziert werden, die in künftigen Zuchtprogrammen Anwendung finden können. In den Untersuchungen im Gewächshaus wies eine Vielzahl von Akzessionen aus dem Kohl-Formenkreis ein erhöhtes Resistenzniveau auf, was vorherige Ergebnisse bestätigt. Des Weiteren konnten wir zeigen, dass das getestete B. rapa- Sortiment vornehmlich einen niedrigen bis moderaten Resistenzphänotyp besaß, während die getesteten napus- Typen in der Regel hinsichtlich ihrer Resistenz eine Mittelstellung einnahmen. Obwohl zwischen den Ergebnissen aus den Gewächshaus- Untersuchungen und den 
Beobachtungsprüfungen im Freiland nur eine geringe Gesamtkorrelation bestand, erwies sich die Kombination beider Verfahren als geeignet, um robuste Resistenzquellen zu identifizieren. Ob niedrige Befallswerte im Feld hierbei auf im Spross greifende Toleranzbzw. Resistenzmechanismen zurückzuführen sind oder sich zumindest teilweise mit der späten Abreife der jeweiligen Akzession erklären lassen, womit es sich um eine Scheinresistenz handeln würde, ist weiterhin unklar.

Um Mechanismen zu charakterisieren, die zu dem Resistenzphänotypen von Brassica-Arten gegenüber V. longisporum beitragen und für Rapszüchter potentiell nutzbar sind, wurden grundsätzlich zwei verschiedene Ansätze verfolgt. Zum einen sollte versucht werden, effiziente Resistenzfaktoren in Inokulationen mit virulenten Isolaten von $V$. longisporum gegenüber wirtsheterologen Stämmen von V. dahliae zu charakterisieren. So wurde die Interaktion von $V$. longisporum bzw. $V$. dahliae mit Raps im Bereich der Wurzeln und im vaskulären System des Sprosses mit Hilfe der Confocalen Laser Scanning Mikroskopie (CLSM) unter Verwendung sowohl GFP-markierter Stämme als auch konventioneller Fluoreszenzfarbstoffe analysiert. Die Ergebnisse dieser Studien ergaben eine bessere Eignung der Fluoreszenzfarbstoffe im Vergleich zu der Verwendung von GFP als konstitutiv exprimierten Markerprotein im Rahmen von Wirt- Parasit- Interaktionsstudien. Des Weiteren lieferten diese Untersuchungen neue Informationen zur Frühphase in der Wirt- und NichtwirtPathogen- Interaktion. Real- time PCR- Untersuchungen im Verlauf der Pathogenese zeigten, dass $V$. dahliae nicht in der Lage war, sich systemisch in den Spross auszubreiten, obwohl Infektionen der Wurzeln stattfanden. Die Nichtwirtsresistenz von Raps gegenüber V. dahliae beruht somit auf Mechanismen, welche die systemische Ausbreitung des Pilzes hemmen.

Des Weiteren wurde Screeningmaterial mit vorhandenen Resistenzunterschieden zur differentiellen Charakterisierung von Resistenzmechanismen herangezogen. Unter den ausgewählten Genotypen befanden sich ein resistenter Kohl und ein anfälliger sowie ein resistenter Rapsgenotyp. Auf der Grundlage der Ergebnisse der Untersuchungen der Nichtwirt- Interaktion standen Resistenzmechanismen im vaskulären System im Mittelpunkt. Im Rahmen dieser Untersuchungen wurden Mechanismen identifiziert, die in Kraft treten, nachdem das Pathogen in die Pflanze eingedrungen ist. So wurde V. longisporum bei der Interaktion mit einem resistenten Wirt auf den unteren Bereich des Stängels begrenzt, eine akropetale Ausbreitung in den Spross war nicht möglich. Ein Aspekt der Resistenz von Brassica spp. gegenüber Verticillium manifestiert sich somit als Ausbreitungsresistenz. Histologische Untersuchungen der wirtspezifischen Interaktion führten zu der Identifikation folgender unspezifischer Abwehrmechanismen: Bildung von Thyllen und/oder vaskulärer 
Gele, Akkumulation von Phenolen im Xylemparenchym und verstärkte Lignifizierung der Zellwände des Xylems und des Xylemparenchyms. Die Beteiligung von phenolischen Stoffen während der Abwehr konnte durch quantitative biochemische Analysen bestätigt werden. Bei der Ausprägung aller genannten Prozesse zeigte sich ein positiver Zusammenhang zwischen dem Resistenzniveau des betrachteten Genotyps und der Stärke der Reaktion (Gefäßverschluss) bzw. dem Gehalt der betrachteten Komponenten. Bei der Resistenz von Brassica spp. handelt es sich somit um eine quantitative Resistenz. Sowohl in dem resistenten Kohl- Genotypen als auch der resistenten Raps- Akzession traten Abwehrreaktionen am stärksten im Hypokotyl zutage und schienen zumindest zum Teil auf den gleichen Mechanismen zu beruhen; dies stützt die Annahme, das Resistenzmerkmale von B. oleracea auf B. napus im Laufe interspezifischer Hybidisierungen zwischen B. rapa und B. oleracea übertragen worden sind.

Die Ergbnisse dieser Arbeit sind ein erster Schritt auf dem Weg zum Verständnis der biochemischen Grundlagen der genetisch determinierten Resistenz von Raps genüber $V$. longisporum. Die Kombination von Fragestellungen der Pflanzenpathologie und der Pflanzenzüchtung in einem integrierten Ansatz kann dazu beitragen, Merkmale zu selektieren, die züchterisch nutzbar sind, mit dem Ziel, die Sortenresistenz von Winterraps gegenüber $V$. longisporum zu verbessern. 


\section{References}

Ampomah, Y.A., Friend, J. (1988) Insoluble phenolic compounds and resistance of potato tuber discs to Phytophthora and Phoma. Phytochemistry 27, 2533-2541

Anterola, A.M., Lewis, N.G. (2002) Trends in lignin modification: a comprehensive analysis of the effects of genetic manipulations/mutations on lignification and vascular integrity. Phytochemistry 61, 221-294

Atibalentja, N., Eastburn, D.M. (1998) Verticillium dahliae resistance in horseradish germ plasm from the University of Illinois collection. Plant Disease 82, 176-180

Baayen, R.P., Van Eijk, C., Elgersma, D.M. (1989) Histology of roots of resistant and susceptible carnation cultivars from soil infested with Fusarium oxysporum f. sp. dianthi. Netherland Journal of Plant Pathology 95, 3-13

Babadoost, M., Chen, W., Bratsch, A.D., Eastman, C.E. (2004) Verticillium longisporum and Fusarium solani: two new species in the complex of internal discoloration of horseradish roots. Plant Pathology 53: 669-676

Baidez, A.G., Gomez, P., Del Rio, J.A., Ortuno, A. (2007) Dysfunctionality of the xylem in Olea europaea L. plants associated with the infection process by Verticillium dahliae Kleb. Role of phenolic compounds in plant defense mechanism. Journal of Agricultural and Food Chemistry 55, 3373-3377

Banuett F. (1995) Genetics of Ustilago maydis, a fungal pathogen that induces tumors in maize. Annual Review of Genetics 29, 179-208.

Barabara, D.J., Clewes, E. (2003) Plant pathogenic Verticillium species: how many of them are there? Molecular Plant Pathology 4, 287-305

Barabara, D.J., Morton, A., Miller, N.J. (2005) Isolation of micro satellite markers from an interspecific hybrid isolate of the fungal plant pathogen Verticillium dahliae. Molecular Ecology Notes 5, 857-859

Baranowski, J.D., Davidson, P.M., Nagel, C.W., Branen, A.L. (1980) Inhibition of Saccaromyces cerevisiae by naturally occurring hydroxycinnamates. Journal of Food Science $45,592-594$

Barber, M.S., Mitchell, H.J. (1997) Regulation of phenylpropanoid metabolism in relation to lignin biosynthesis in plants. International Review of Cytology 172, 243-293

Barz, W., Hoesel, W. (1979) Metabolism and degradation of phenolic compounds in plants. In: Swain, T., Harborne, J.B., Van Sumere, C.F. (eds.) Biochemistry of Plant Phenolics. New York and London: Plenum Press, pp. 339-369

Becker, H.C., Engquist, G.M., Karlssom, B. (1995) Comparison of rapeseed cultivars and resynthesized lines based on allozyme and RFLP markers. Theoretical and Applied Genetics $91,62-67$ 
Beckman, C.H. (1964) Host responses to vascular infection. Annual Review of Phytopathology 2, 231-252

Beckman, C.H. (1966) Cell irritability and localization of vascular infections in plants. Phytopathology 56, 821-824

Beckman, C.H. (1969) The mechanics of gel formation by swelling of simulated plant cell wall membranes and perforation plates of banana root vessels. Phytopathology 52, 893-897

Beckman, C.H. (1987) The nature of wilt diseases of plants. The American Phytopathological Society, St Paul, MN

Beckman, C.H. (1990) Host responses to the pathogen. In: Fusarium wilt of the banana. Ploetz, R.C. (ed.) The American Phytopathological Society, St Paul, MN. Pp. 107-114

Beckman, C.H. (2000) Phenolic-storing cells: Keys to programmed cell death and periderm formation in wilt disease resistance and in general defence responses in plants? Physiological and Molecular Plant Pathology 57, 101-110

Beckman, C.H., Elgersma, D.M., Mac Hardy, W.E. (1972) The localization of fusarial infections in the vascular tissue of single-dominant-gene resistant tomatoes. Phytopathology $62,1256-1260$

Beckman, C.H., Halmos, S. (1962) Relation of vascular occluding reactions in banana roots to pathogenicity of root-invading fungi. Phytopathology 52, 893-897

Beckman, C.H., Halmos, S., Mace, M.E. (1962) The interaction of host, pathogen, and soil temperature in relation to susceptibility to Fusarium wilt of bananas. Phytopathology 52, 134140

Beckman, C.H., Müller, W.C., Mace, M.E. (1974) The stabilization of artificial and natural cell wall membranes by phenolic infusion and its relation to wilt resistance. Phytopathology $64,1214-1220$

Beckman, C.H., Müller, W.C., Mac Hardy, W.E. (1972) The localization of stored phenols in plant hairs. Physiological Plant Pathology 2, 69-74

Beckman, C.H., Roberts, E. (1995) On the nature and the genetic basis for resistance and tolerance to fungal wilt diseases of plants. Advances in Botanical Research 21, 35-77

Beckman, C.H., Talboys, P.W. (1981) Anatomy of resistance. In: Fungal wilt diseases of plants. Mace, M.E., Bell, A.A., Beckman, C.H. (eds.) Academic Press, New York, pp. 487521

Beckman, C.H., Zaroogian, G.E. (1967) Origin and composition of vascular gel in infected banana roots. Phytopathology 57, 11-13

Beckmann CH (1987) The Nature of Wilt Diseases of Plants. St. Paul, MN, USA: APS Press

Bell, A.A. (1969) Phytoalexin production and Verticillium resistance in cotton. Phytopathology 59, 1119-1127 
Bell, A.A. (1970) 4-Hydroxybenzaldehyde and vanillin as toxins formed in leaf wound sap of Phaseolus lunatus. Phytopathology 60, 161-165

Bell, A.A. (1992) Verticllium wilt. In: Cotton diseases. Hillocks, R.J. (ed.) CAB International, Wallingford, U.K. pp. 87-126

Bell, A.A. (1994) Mechanisms of disease resistance in Gossypium species and variation in Verticillium dahliae. In: Constable, G.A., Forrester, N.W. (eds.) Challenging the future: Proceedings of the World Cotton Conference 1, Brisbane, Australia

Benhamou, N., Garand, C. (2001) Cytological analysis of defense-related mechanisms induced in pea root tissues in response to colonization by nonpathogenic Fusarium oxysporum Fo47. Phytopathology 91, 730-740

Bernards, M.A., Lewis, N.G. (1992) Alkyl ferulates in wound healing potato tubers. Phytochemistry 31, 3409-3412

Bevan M (1984) Binary Agrobacterium vectors for plant transformation. Nucleic Acids Research 12: 8711-8721

Bewley, W.F. (1922) Sleepy disease of the tomato. Annals of Applied Biology 9, 116-134

Bhat RG, Subbarao KV (1999) Host range specificity in Verticillium dahliae. Phytopathology 89: $1218-1225$

Bishop, C.D., Cooper, R.M. (1983a) An ultrastructural study of root invasion in three vascular wilt diseases. Physiological Plant Pathology22, 15-27

Bishop, C.D., Cooper, R.M. (1984) Ultrastructure of vascular colonization by fungal wilt pathogens. II. Invasion of resistant cultivars. Physiological Plant Pathology 24, 277-289

Bolek, Y., El-Zik, K.M., Pepper, A.E., Bell, A.A., Magill, C.W., Thaxton, P.M., Reddy, O.U.K. (2005) Mapping of Verticillium wilt resistance in cotton. Plant Science 168, 15811590

Bolwell, G.P., Robbins, M.P., Dixon, R.A. (1985) Metabolic changes in elicitor-treated bean cells. European Journal of Biochemistry 148, 571-578

Bolwerk A, Lagopodi A, Lugtenberg BJJ, Bloemberg GV (2005) Visualization of interactions between a pathogenic and a benefical Fusarium strain during biocontrol of tomato foot and root rot. Molecular Plant-Microbe Interactions 18: 710-721

Boudet, A.M., Lapierre, C. and Grima-Pettenati, J. (1995) Biochemistry and molecular biology of lignification. New Phytologist 129, 203-236.

Bowers, J.H., Nameth, S.T., Riedel, R.M., Rowe, R.C. (1996) Infection and colonization of potato roots by Verticillium dahliae as affected by Pratylenchus penetrans and P. crenatus. Phytopathology 86, 614-621

Brandt, W.H., Lacy, M.L., Horner, C.E. (1984) Distribution of Verticillium in stems of resistant and susceptible species of mint. Phytopathology 74, 587-591 
Brasier, C.M., Cooke, D.E.L., Duncan, J.M. (1999) Origin of a new Phytophthora pathogen through interspecific hybridisation. Proceedings of the National Academy of Science USA, $96,5878-5883$

Bruce, R.J., West, C.A. (1989) Elicitation of lignin biosynthesis and isoperoxidase activity by pectic fragments in suspension cultures of Castor bean. Plant Physiology 91, 889-897

Buckley P.M., Wyllie T.D., DeVay J.E. (1969) Fine structure of conidia and conidium formation in Verticillium albo-atrum and V. nigrescens. Mycologia 61: 240-250

Bundock P., den Dulk-Ras A., Beijersbergen A., Hoykaas P.J.J. (1995) Trans-kingdom TDNA transfer from Agrobacterium tumefaciens to Saccharomyces cerevisiae. European Molecular Biology Organization 14: 3206-3214

Cahill, D.M., Bennett, I.J., McComb, J.A. (1993) Mechanisms of resistance to Phytophthora cinnamoni in clonal, micropropagated Eucalyptus marginata. Plant Pathology 42, 865-872

Campbell C.L., Madden L.V. (1990) Introduction to Plant Disease Epidemiology. New York, USA. John Wiley.

Campbell, M.M., Ellis, B.E. (1992a) Fungal elicitor-mediated responses in pine cell cultures. I. Induction of phenylpropanoid metabolism. Planta 186, 409-417

Campbell, M.M., Ellis, B.E. (1992b) Fungal elicitor-mediated responses in pine cell cultures: cell wall-bound phenolics. Phytochemistry $31,737-742$

Candela, M.E., Alcázar, M.D., Espín, A., Egea, C., Almela, L (1995) Soluble phenolic acids in Capsicum annuum stems infected with Phytophthora capsici. Plant Pathology 44, 116-123

Cernusko, R. (1995) Entwicklung und Erprobung enzymatischer und immunologischer Nachweismethoden für Verticillium dahliae Kleb. im Raps (Brassica napus). Dissertation, Georg-August-Universität Göttingen

Cernusko, R., Wolf, G.A. (1997) Immunological methods for the detection of Verticillium dahliae in oilseed rape. In: Dehne, H.-W., Adam, G., Diekman, M., Frahm, J., MaulerMachnik, A, van Halteren, P. (eds.) Diagnosis and identification of plant pathogens. Kluwer Academic Publishers, Dordrecht, Boston, London

Chalfie M, Kain S (1998) Green Fluorescent Protein. Properties, Applications and Protocols. Wiley-Liss, Inc., New York

Chambers, H. L., Corden, M.E. (1963) Semeiography of Fusarium wilt of tomato. Phytopathology 53, 1006-1010

Chattaway, M.M. (1949) The development of tyloses and secretion of gum in heartwood formation. Australian Journal of Scientific Research Series B, Biological Science 2, 227-240

Chen, A., Chai, Y., Li, J., Chen, L. (2007) Molecular cloning of two genes encoding cinnamate 4-hydoxylase $(\mathrm{C} 4 \mathrm{H})$ from oilseed rape (Brassica napus). Journal of Biochemistry and Molecular Biology 40, 247-260 
Chen, P., Lee, B., Robb, J. (2004) Tolerance to a non-host isolate of Verticillium dahliae in tomato. Physiological and Molecular Plant Pathology 64, 283-291

Chong, J., Baltz, R., Fritig, B., Saindrenan, P. (1999) An early salicylic acid-, pathogen- and elicitor-inducible tobacco glucosyltransferase: role in compartmentalization of phenolics and $\mathrm{H}_{2} \mathrm{O}_{2}$ metabolism. FEBS Letters 458, 204-208

Clark, M.F., Adams, A.N. (1977) Characteristics of the microplate method of enzyme-linked immunosorbent assay for the detection of plant viruses. Journal of General Virology 34, 475483

Clewes, E., Barbara, D.J. (2005) Hopeful monsters. In: Program and Book of Abstracts of the Ninth International Verticillium Symposium. California, USA, 17-21 June, p.18

Collins, A., Okoli, A.N., Morton, A., Parry, D., Edwards, S.G., Barbara, D.J. (2003) Isolates of Verticillium dahliae pathogenic to crucifers are of at least three distinct molecular types. Phytopathology 93, 364-376

Concibido, V.C., Secor, G.A., Jansky, S.H. (1994) Evaluation of resistance to Verticillium wilt in diploid, wild potato interspecific hybrids. Euphytica 76, 145-152

Conway, W.S., MacHardy, W.E. (1978) Distribution and growth of Fusarium oxysporum f. sp. lycopersici race 1 or race 2 within tomato plants resistant or susceptible to wilt. Phytopathology 68, 938-942

Cooper, R.M., Resende, M.L.V., Flood, J., Rowan, M.G., Beale, M.H., Potter, U. (1996) Detection and cellular localization of elemental sulphur in disease resistant genotypes of Theobroma cacao. Nature 246, 309-311

Cooper, R.M., Williams, J.S. (2004) Elemental sulphur as an induced antifungal substance in plant defence. Journal of Experimental Botany 55, 1947-1953

Cooper, R.M. (1999) Verticillium-Host Interactions: past achievements and future molecular prospects. In: Advances in Verticillium research and disease management. Tjamos, E.C., Rowe, R.C., Heale, J.B., Fravel, D.R. (eds.), St. Paul, Minnesota, APS Press, pp 144-150

Corsini, D.L., Pavek, J.J., Davis, J.R. (1988) Verticillium wilt resistance in noncultivated tuber-bearing Solanum species. Plant Disease 72, 148-151

Cottle, W., Kolattukudy, P.E. (1982) Biosynthesis, deposition, and partial characterization of potato suberin phenolics. Plant Physiology 69, 393-399

Covert SF, Kapoor P, Lee M Briley A, Nairn CJ (2001) Agrobacterium tumefaciens-mediated transformation of Fusarium circinatum. Mycological Research 105: 259-264

Crouch, J.H., Lewis, B.G., Mithen, R.F. 81994) The effect of A genome substitution on the resistance of $B$. napus to infection by Leptosphaeria maculans. Plant Breeding 112, 265-278

Czapek, F. 1902. Untersuchungen über die Stickstoffgwinnung und Eiweißbildung der Pflanzen. Breitr Chem Physiol Pathol 2:540-560. 
Daayf, F. Nicole, M., Boher, B., Pando, A., Geiger, J.P. (1997) Early vascular defence reactions of cotton roots infected with defoliating mutant strain of Verticillium dahliae. European Journal of Plant Pathology 103, 125-136

Daebeler F, Amelung D, Zeise K (1988) Verticillium-Welke an Winterraps - Auftreten und Bedeutung. Nachrichtenblatt Pflanzenschutzdienst DDR 42: 71-73

Davis, D., Dimond, A.E. (1954) The source and role of phenols in Fusarium wilt symptoms. Phytopathology 44: 485-486

Davis, D, Waggoner, P.E., Dimond, A.E. (1953) Conjugated phenols in the Fusarium wilt syndrome. Nature 172: 959-961

De Ascensao, A.R. F. D.C., Dubery, I.A. (2003) Soluble and cell wall-bound phenolics and phenolic polypmers in Musa acuminata roots exposed to elicitors from Fusarium oxysporum f. sp. cubense. Phytochemistry 63, 679-686

De Ascensao, A.R.D.C.F., Dubery, I.A. (2000) Panama disease: cell wall reinforcement in banana roots in response to elicitors from Fusarium oxysporum f. sp. cubense race four. Phytopathology 90: 1173-1180

Debode, J., Declercq, B., Höfte, M. (2005) Identification of cauliflower cultivars that differ in susceptibility to Verticillium longisporum using different inoculation methods. Journal of Phytopathology 153, 257-263

Deese, D.C., Stahmann, M.A. (1962a) Pectic enzymes and cellulase formation by Fusarium oxysporum f. sp. cubense on stem tissues from resistant and susceptible banana plants. Phytopathology 52, 247-255

Deese, D.C., Stahmann, M.A. (1962b) Pectic enzymes in Fusarium-infected susceptible and resistent tomato plants. Phytopathology 52, 255-260

De Lorenzo, G., Cervone, F. (1997) Polygalacturonase-inhibiting proteins (PGIP): their role in specificity and defense against pathogenic fungi. In: Stacey, G., Kenn, N.T. (eds.) Plantmicrobe interactions, Chapman \& Hall, New York, vol 3, pp. 76-93

De Lorenzo, G., D’Ovidio, R., Cervone, F. (2001) The role of polygalacturonase-inhibiting proteins (PGIPs) in defense against pathogenic fungi. Annual Review of Phytopathology 39, 313-335

Dickinson, C.H., Lucas, J.A. (1987) Interacción hospedero-patógeno a nivel molecular. In: Patología Vegetal y Patógenos de Plantas (ISBN 968-18-21117-3). Limusa, México: 187-228

Diederichsen, E., Sacristan, M.D. (1996) Disease response of resynthesized Brassica napus L. lines carrying different combinations of resistance to Plasmodiophora brassicae. Plant Breeding 115, 5-10

Dimond AE (1970) Biophysics and biochemistry of the vascular wilt syndrome. Annual Review of Phytopathology 8: 301-322 
Diwan, N., Fluhr, R., Eshed, Y., Zamir, D., Tanksley, S.D. (1999) Mapping of Ve in tomato: a gene conferring resistance to the broad-spectrum pathogen, Verticillium dahliae, race 1. Theoretical and Applied Genetics 98, 315-319

Dixelius, C., Happstadius, I., Berg, G. (2005) Verticillium wilt on Brassica oil crops - a swedish perspective. Journal of the Swedish Seed Association 115: 36-48.

Dixon G.R., Pegg G.F. (1969) Hyphal lysis and tylose formation in tomato cultivars infected by V. albo-atrum. Transactions of the British Mycological Society 53, 109-118

Dixon G.R., Pegg G.F. (1972) Changes in the amino acid content of tomato xylem sap following infection with strains of Verticillium albo-atrum. Annals of Botany 36: 147-154

Dixon, R.A., Lamb, C.J., Masoud, S., Sewalt, V.J.H., Paiva, N.L. (1996) Metabolic engineering: prospects for crop improvement through the genetic manipulation of phenylpropanoid biosynthesis and defence responses - a review. Gene 179, 61-71

Dixon, R.A., Paiva, N.L. (1995) Stress-induces phenylpropanoid metabolism. Plant Cell 7, 1085-1097

Dixon, R.A., Steele, C.L.(1999) Flavonoids and isoflavonoids - a gold mine for genetic engineering. Trends in Plant Science 4, 394-400

Dobinson K.F. (1994) Genetic transformation of the vascular wilt fungus Verticillium dahliae. Canadian Journal of Botany, 73: 710-715

Domsch KH, Gams W Anderson T-H (1980) Nectria (Fr) 1849, Verticillium Nees ex Link 1824. In: Compendium of Soil Fungi, Vol 1, 829-845. Academic Press, New York.

Dox, A. W. 1910. The intracellular enzymes of Penicillium and Aspergillus with special references to those of P. camenberti. US Dept Agric Bur Anim Ind Bull 120:1-170.

Dreyer, F., Graichen, K., Jung, C. (2001) A maljor quantitative trait locus for resistance to turnip yellows virus (TuYV, syn. beet western yellows virus, BWYV) in rapeseed. Plant Breeding 120, 457-462

Dubery, I.A., Slater, V. (1997) Induced defence responses in cotton leaf disks by elicitors from Verticillium dahliae. Phytochemistry 44, 1429-1434

Dubery, I.A., Smit, F. (1994) Phenylalanine ammonia-lyase from cotton (Gossypium hirsutum) hypocotyls: properties of the enzyme induced by a Verticillium dahliae induced phytotoxin. Biochem. Biophys. Acta 1207, 24-30

Dunker, S., Keunecke, H., von Tiedemann, A. (1996) Verticillium longisporum in winter oilseed rape - Impact on plant development and yield. IOBC/wprs Bulletin Vol. 29(x), 365374

Eastburn, D.M., Chang, R.J. (1994) Verticillium dahliae: a causal agent of root discoloration of horseradish in Illinois: Plant Disease 78: 496-498 
Eckert M, Maguire K, Urban M, Foster S, Fitt B, Lucas J, Hammond-Kosack K (2005) Agrobacterium tumefaciens-mediated transformation of Leptosphaeria spp. and Oculimacula spp. with the reef coral gene DsRed and the jellyfish gene gfp. FEMS Microbiology Letters 253: $67-74$

Elgersma, D.M. (1970) Length and diameter of xylem vessels as a factor of resistance of elms to Ceratocystis ulmi. Netherland Journal of Plant Pathology 76, 179-182

Elgersma, D.M., Machardy, W.E., Beckman, C.H. (1972) Growth and distribution of Fusarium oxysporum f. sp. lycopersici in near-isogenic lines of tomato resistant and susceptible to wilt. Phytopathology 62, 1232-1237

EUROSTAT: http://epp.eurostat.ec.europa.eu

Eynck C, Koopmann B, Grunewaldt-Stöcker G, Karlovsky P, von Tiedemann A (2007) Differential interactions of Verticillium longisporum and Verticillium dahliae with Brassica napus detected with molecular and histological techniques. European Journal of Plant Pathology 118, 259-274

Fähling, M., Graf, H., Siemens, J. (2003) Pathotype separation of Plasmodiophora brassicae by the host plant. Journal of Phytopathology 151, 425-430

Fahleson J, Lagercrantz U, Hu Q, Steventon LA, Dixelius C (2003) Estimation of genetic variation among Verticillium isolates using AFLP analysis. European Journal of Plant Pathology 109: 361-371

Fahleson, J., Hu, Q., Dixelius, C. (2004) Phylogenetic analysis of Verticillium species based on nuclear and mitochondrial sequences. Archives of Microbiology 181, 435-442

Farmer, E.E. (1985) Effects of fungal elicitor on lignin biosynthesis in cell suspension cultures of soybean. Plant Physiology 78, 338-342

Feder, N., O`Brien, T.P. (1968) Plant microtechniques: Some principles and new methods. American Journal of Botany 55, 123-142

Fergus, B.J., Goring, D.A.I. (1970) The location of guaiacyl and syringyl lignins in birch xylem tissue. Holzforschung 24, 113-117

Fillingham, A.J., Wood, J., Bevan, J.R., Crute, I.R., Mansfield, J.W., Taylor, J.D., Vivian, A. (1992) Avirulence genes from Pseudomonas syringae pathovars phaseolicola and pisi confer specificity towards both host and non-host species. Physiological and Molecular Plant Pathology 40: 1-15

Fisch, M.H., Flick, B.H., Arditti, J. (1973). Structure and antifungal activity of hircinol, loroglossol and orchinol. Phytochemistry 12, 437-441

Fradin, E.F., Thomma, B.P.H.J. (2006) Physiology and molecular aspects of Verticllium wilt diseases caused by V. dahliae and V. albo-atrum. Molecular Plant Pathology 7, 71-86

Friend, J. (1976) Lignification in infected tissue. In: Biochemical Aspects of Plant-Parasite Relationships. Friend, J., Threfall, D.R. (eds.) Academic Press, New York, pp. 291-303 
Friend, J., Reynolds, S.B., Aveyard, A. (1973) Phenylalanine ammonia lyase, chlorogenic acid, and lignin in potato tuber tissue inoculated with Phytophthora infestans. Physiological Plant Pathology 3, 495-507

Fry, S.C. (1982) Phenolic components of the primary cell wall. Biochemical Journal 203, 493-504

Fry, S.C. (1987) Intracellular feruloylation of pectic polysaccharides. Planta 171, 205-211.

Fry, S.C. (1993) Feruloylated pectins from the primary cell wall: their structure and function. Planta 157, 111-123

Fuchs, H. \& Sacristan, M.D. (1996) Identification of a gene in Arabidopsis thaliana controlling resistance to clubroot (Plasmodiophora brassicae) and characterization of the resistance response. Molecular Plant-Microbe Interaction 9, 91-97

Gams W, Zare R (2001) A revision of Verticillium sect. Prostrata. III. Generic classification. Nova Hedwigia 72:329-337

Gao, H., Beckman, C.H., Mueller, W.C. (1995a) The rate of vascular colonization as a measure of the genotypic interaction between various cultivars of tomato and various formae or races of Fusarium oxysporum. Physiological and Molecular Plant Pathology 46, 29-43

Gao, H., Beckman, C.H., Mueller, W.C. (1995b) The nature of tolerance to Fusarium oxysporum f.sp. lycopersici in polygenically field-resistant marglobe tomato plants. Physiological and Molecular Plant Pathology 46, 401-412

Garber, R.H., Houston, B.R. (1966) Penetration and development of Verticillium albo-atrum in the cotton plant. Phytopathology 56, 993-1118

Garas, N.A., Wilhelm, S., Sagen, J.E. (1986) Relationship of cultivar resistance to distribution of Verticillium dahliae in inoculated cotton plants and to growth of single conidia on excised stem segments. Phytopathology 76, 1005-1010

Gazendam, I., Oelofse, D., Berger, D.K. (2004) High-level expression of apple PGIP1 is not sufficient to protect transgenic potato against Verticillium dahliae. Physiological and Molecular Plant Pathology 65, 145-155

Gold J., Lee B., Robb J. (1996) Colonization of tomatoes by Verticillium dahliae: determinative phase II. Canadian Journal of Botany 74: 1279-1288

Gold, J., Robb, J. (1995) The role of the coating response in Craigella tomatoes infected with Verticillium dahliae, races 1 and 2. Physiological and Molecular Plant Pathology 47, 141-157

Goodman, R.N., Király, Z., Wood, K.R. (1986) The Biochemistry and Physiology of Plant Disease. University of Missouri Press, Columbia

Goodman, R.N., Novacky, A.J. (1994) The Hypersensitive Reaction in Plants to Pathogens. St. Paul: APS Press 
Gordon, S.A., Paleg, L.G. (1961) Formation of auxin from tryptophan through action of polyphenols. Plant Physiology 36, 838-845

Gordon, T.R., Kirkpatrick, S.C., Hansen, J., Shaw, D.V. (2006) Response of strawberry genotypes to inoculation with isolates of Verticillium dahliae differing in host origin. Plant Pathology 55, 766-769

Gothoskar, S.S., Scheffer, R.P., Walker, J.C., Stahmann, M.A. (1955) The role of enzymes in the development of Fusarium wilt of tomato. Phytopathology 45, 381-387

Graham, T.E. (1998) Flavanoid and flavanol glycosid metabolism in Arabidopsis. Plant Physiology and Biochemistry 36, 135-144

Green RJJ (1981) An overview. In: Mace ME, Bell AA, Beckman CH (eds.) Fungal Wilt Diseases of Plants. Academic Press, New York, pp. 1-24

Griffiths, D.A. (1971) The development of lignitubers in roots after infection by Verticillium dahliae Kleb. Canadian Journal of Microbiology 17, 441-444

Günzelmann, H., Paul, V.H. (1990) Zum Auftreten und zur Bedeutung der VerticilliumWelke an Raps in der Bundesrepublik Deutschland in 1989. Raps 8: 23-25

Gulaya, T.J., Sackston, W.E., Viranyi, F., Masirevic, S., Rashid, K.Y. (1991) New races of the sunflower downy mildew interaction pathogen (Plasmopara helstedii) in Europe and North and South America. Journal of Phytopathology 132, 303-311

Hahlbrock, K, Scheel, D. (1989) Physiology and molecular biology of phenylpropanoid metabolism. Annual Review of Plant Physiology and Plant Molecular Biology 40, 347-369

Hamer JE, Talbot NJ. (1998) Infection-related development in the rice blast fungus Magnaporthe grisea. Current Opinions in Microbiology 1:693-7

Hammerschmidt, R. (1983) Rapid deposition of lignin in potato tuber tissue as a response to fungi non-pathogenic on potato. Physiological Plant Pathology 24, 33-42

Hammerschmidt, R. (2005) Phenols and plant-pathogen interactions: the saga continues. Physiological and Molecular Plant Pathology 66, 77-78

Hammond-Kosack, K.E., Jones, J.D.G. (1996) Resistance gene-dependent plant defense responses. Plant Cell 8, 1773-1791

Hanahan J. (1983) Studies on transformation of Escherichia coli with plasmids. Journal of Molecular Biology 166: 557-580

Happstadius I., Ljunberg A., Kristiansson B., Dixelius C. (2003) Identification of Brassica oleracea germplasm with improved resistance to Verticillium wilt. Plant Breeding 122: 30-34

Harakava, R. (2005) Genes encoding enzymes of the lignin biosynthesis pathway in Eucalyptus. Genetics and Molecular Biology 28, 601-607 
Hasan, M., Seyis, F., Badani, A.G., Pons-Kuhnemann, J., Lühs, W., Friedt, W., Snowdon, R.J. (2006) Surveying genetic diversity in the Brassica napus gene pool using SSR markers. Genetic Resources and Crop Evolution 53, 793-802

Hastie, A.C., Heale, J.B. (1981) Genetics of Verticillium. In: Crulli, M. (ed.) Pathobiology of Verticillium species. Mediterranean Phytopathological Union, Firenze, Italy, pp. 36-68

Heale J.B., Karapapa V.K. (1999) The Verticillium threat to Canada's major oilseed crop: Canola. Canadian Journal of Plant Pathology 21:1-7

Heale, J.B. (2000) Diversification and speciation in Verticillium - An overview. In: Advances in Verticillium research and disease management. Tjamos, E.C., Rowe, R.C., Heale, J.B., Fravel, D.R. (eds.), St. Paul, Minnesota, APS Press, pp. 175-177

Heale, J.B., Karapapa, K.V. (1999) The Verticillium threat to Canada's major oilseed crop: canola. Canadian Jouranl of Plant Pathology 21, 1-7

Heath, M.C. (1985) Implications of non-host resistance for understanding host-parasite interactions. In: Groth, J.V., Bushnell, W.R. (eds.) Genetic Basis of Biochemical Mechanisms of Plant Disease. St. Paul: APS Press, pp. 25-42

Heath, M.C. (1997) Evolution of plant resistance and susceptibility to fungal parasites. In: Carroll, G.C., Tudzynski, P (eds.) The Mycota V, Part B, Plant Relationships. Berlin: Springer, pp. 257-276

Heath, M.C. (2000) Nonhost resistance and nonspecific plant defenses. Current Opinion in Plant Biology 3, 315-319

Heath, M.C. (2001) Non-host resistance to plant pathogens: nonspecific defense or the result of specific recognition events? Physiological and Molecular Plant Pathology 58, 53-54

Heinz, R., Lee, S.W., Saparno, A., Nazar, R.N., Robb, J. (1998) Cyclical systemic colonization in Verticillium-infected tomato. Physiological and Molecular Plant Pathology 52, 385-396

Hermanns, M., Slusarenko, A.J., Schlaich, N.L. (2003) Organ-specificity in a plant disease is determined independently of $\mathrm{R}$ gene signaling. Molecular Plant-Microbe Interactions 16, $752-$ 759

Hill, M.K., Lyon, K.J., Lyon, B.R. (1999) Identification of disease response genes expressed in Gossypium hirsutum upon infection with the wilt pathogen Verticillium dahliae. Plant Molecular Biology 40, 289-296

Hood, E.E., Helmer, G.L., Fraley, R.T., Chilton, M.D. (1986) The hypervirulence of Agrobacterium tumefaciens A281 is encoded in a region of pTiBo542 outside of T-DNA. Journal of Bacteriology 168: 1291-1301

Horowitz, S., Freeman, S., Sharon, A. (2002) Use of green fluorescent protein-transgenic strains to study pathogenic and nonpathogenic lifestyles in Colletotrichum acutatum. Phytopathology 92: 743-749 
Huisman, O.C. (1982) Interrelations of root growth dynamics to epidemiology of rootinvading fungi. Annual Review of Phytopathology 20, 303-327

Humphreys, J.M., Chapple, C. (2002) Rewriting the lignin road map. Current Opinion in Plant Biology 5, 224-229

Hunter, D.E., Darling, H.M., Stevenson, F.J., Cunningham, C.E. (1968) Inheritance of resistance to Verticillium wilt in Wisconsin. American Potato Journal 45, 72-78

Iiyama, K., Lam, T.B.T., Stone, B.A. (1990) Phenolic acid bridges between polysaccharides and lignin in wheat internodes. Phytochemistry 29, 733-737

Iiyama, K., Lam, T.B.T., Stone, B.A. (1994) Covalent cross-links in the cell wall. Plant Physiology 104, 315-320

Isaac, I. (1957) Verticillium wilt of Brussels sprout. Annals of Applied Biology 45: 276-283

James, J.T. \& Dubery, I.A. (2001) Inhibition of polygalacturonase from Verticillium dahliae by a polygalacturonase inhibiting protein from cotton. Phytochemistry 57, 149-156

Jansky, S. (2000) Breeding for disease resistance in potato. Plant Breeding Review 19, 69-155

Jansky, S., Rouse, D.I. (2003) Identification of potato interspecific hybrids resistant to Verticillium wilt and determination of criteria for resistance assessment. Potato Research 43, 239-251

Jensen, W.A. (ed., 1962) Botanical histochemistry: Principles and practice. Freeman and Co, San Francisco, California

Johannson, A., Fahleson, J., Zhou, L., Hu, Q., Dixelius, C. (2003) Genomic characterisation of Verticillium longisporum. Eigth International Congress on Plant Pathology. Christchurch, New Zealand, 2-7 Feb 2003, p. 347

Johannson, A., Goud, J.-K., Dixelius, C. (2005) Plant host range of Verticillium longisporum and microsclerotia density in Swedish soils. European Journal of Plant Pathology 114, 139149

Jolivet, P. (1993) Elemental sulfur in agriculture. In: De Kok, L., Stulen, I., Rennenberg, H., Brunold, C., Rauser, W. (eds.) Sulfur nutrition and assimilation in higher plants. The Hague: SPB Academic Publishing, 193-206

Jones, D.H. (1984) Phenylalanine ammonia-lyase: regulation of its induction and its role in plant development. Phytochemistry 23, 1349-1351

Joost, O., Bianchini, G., Bell, A.A., Benedict, C.R., Magill, C.W. (1995) Differential induction of 3-hydroxy-3-methylglutaryl CoA reductase in two cotton species following inoculation with Verticillium. Molecular Plant-Microbe Interactions 8, 880-885

Kamoun, S. (2001) Nonhost resistance to Phytophthora: novel prospects for a classical problem. Current Opinion in Plant Biology 4, 295-300 
Karapapa, VK, Bainbridge, BW, Heale JB (1997) Morphological and molecular characterisation of Verticillium longisporum comb. nov., pathogenic to oilseed rape. Mycological Research 101: 1281- 1294

Karapapa, V.K., Bainbridge, B.W., Heale, J.B. (2000) Classical and molecular characterization of the new species of Verticillium longisporum ( $V$. dahliae var. longisporum Stark 1961; comb. nov. Karapapa et al., 1997) pathogenic to oilseed rape. In: Advances in Verticillium research and disease management. Tjamos, E.C., Rowe, R.C., Heale, J.B., Fravel, D.R. (eds.), St. Paul, Minnesota, APS Press, pp. 196-203

Kawchuk, L.M., Hachey, J., Lynch, D.R., Klcsar, F., van Rooijen, G., Waterer, D.R., Robertson, A., Kokko, E., Byers, E., Howard, R.J., Fischer, R., Prüfer, D. (2001) Tomato Ve disease resistance genes encode cell surface-like receptors. Proceedings of the National Academy of Science USA 98, 6511-6515

Keen, N.T. (1992) The molecular biology of disease resistance. Plant Molecular Biology 19, $109-122$

Kemmochi, I., Kobayashi, I., Tsuchiya, M., Sakai, H., Shimizu, M (2000) Breeding materials for resistance to Verticillium wilt in Japanese cabbage (Brassica oleracea L. var. capitata). Journal of the Japanese Society for Horticultural Science 69, 483-491

Kimber, D.S., McGregor, D.I. (1995) The species and their origin, cultivation and world production. In: Kimber, D.S., McGregor, D.I. (eds.) Brassica Oilseeds. Production and Utilization. CAB International, Cambridge, UK, pp. 1-7

Klement, Z. (1986) Hypersensitivity. In: Phytopathogenic Prokaryotes. Mount, M.S., Lacy, G.H. (eds.) Academic Press, New York, pp. 149-177

Koike, S.T., Subbarao, K.V., Davis, R.M., Gordon, T.R., Hubbard, J.C. (1994) Verticillium wilt of cauliflower in California. Plant Disease 78, 1116-1121

Kolattukudy, P.E. (1981) Structure, biosynthesis and biodegradation of cutin and suberin. Annual Review of Plant Physiology 32, 539-567

Komari T, Halperin W, Nester EW (1986) Physical and functional map of supervirulent Agrobacterium tumefaciens tumor-inducing plasmid pTiBo542. Journal of Bacteriology 166: 88-94

Kosslak, R.M., Dieter, J.R., Ruff, R.L., Chamberlin, M.A., Bowen, B.A., Palmer, R.G. (1996) Partial resistance to root-borne infection by Phytophthora sojae in three allelic necrotic root mutants in soybean. Journal of Heredity 87, 415-422

Kpèmoua, K., Boher, B, Nicole, M, Calatayud, P., Geiger, J.P. (1996) Cytochemistry of defense responses in cassava infected by Xanthomonas campestris pv. manihotis. Canadian Journal of Microbiology 42, 1131-1143

Krüger W (1989) Untersuchungen zur Verbreitung von Verticillium dahliae Kleb. und anderen Krankheits- und Schaderregern bei Raps in der Bundesrepublik Deutschland. Nachrichtenblatt des Deutschen Pflanzenschutzdienstes 41: 49-56 
Kruijt, M., de Kock, M.J.D., de Wit, P.J.G.M. (2005) Receptor-like proteins involved in plant disease resistance. Molecular Plant Pathology 6, 85-97

Lagopodi, A.L., Ram, A.F.J., Lamers, G.E.M., Punt, P.J. (2001) Novel aspects of tomato root colonization and infection by Fusarium oxysporum f. sp. radicis-lycopersici revealed by confocal laser scanning microscopic analysis using the green fluorescent protein as a marker. Molecular Plant-Microbe Interactions 15: 172-179

Lam, T.B.T., Iiyama , K., Stone, B.A. (1992) Cinnamic acid bridges between cell wall polymers in wheat and phalaris internodes. Phytochemistry 31, 1179-1183

Lazo, G.R., Stein, P.A., Ludwig, R.A. (1991) A DNA transformation-competent Arabidopsis genomic library in Agrobacterium. Bio/Technology 9: 963-967

Lee, S.W., Nazar, R.N., Powell, D.A., Robb, J. (1992) Reduced PAL gene suppression in Verticillium-infected resistant tomatoes. Plant Molecular Biology 18, 345-352

Levy, J., Isaac, I. (1976) Colonization of host tissue of varying resistance to Verticillium dahliae. Transactions of the British Mycological Society 67, 91-94

Lewis, N.G., Yamamoto, E. (1990) Lignin: Occurrence, biogenesis and biodegradation. Annu. Rev. Plant Physiol. Plant Mol. Biol. 41, 455-496

Li, R., Rimmer, R., Yu, M., Sharpe, A.G., Séguin-Swartz, G., Lydiate, D., Hegedus, D.D. (2003) Two Brassica napus polygalacturonase inhibitory protein genes are expressed at different levels in response to biotic and abiotic stresses. Planta 217, 299-308

Lorang, J.M., Tuori, R.P., Martinez, J.P., Sawyer, T.L., Redman, R.S., Rollins, J.A., Wolpert, T.J., Johnson, K.B., Rodriguez, R.J., Dickman, M.B., Ciufetti, L.M. (2001) Green fluorescent protein is lighting up fungal biology. Applied and Environmental Microbiology 67: 19871994

Lynch, D.R., Kawchuk, L.M., Hachey, J., Bains, P.S., Howard, R.J. (1997) Identification of a gene conferring high levels of resistance to Verticillium wilt in Solanum chacoense. Plant Disease 81, 1011-1014

Lynkjaer, M.F., Carver, T.L.; Zeyen, R.J. (1997) Suppression of resistance to Erysiphe graminis f.sp. hordei conferred by the mLo5 barley powdery mildew resistance gene. Physiological and Molecular Plant Pathology 36, 63-72

Mace, M.E. (1963) Histochemical localization of phenols in healthy and diseased banana roots. Physiologia Plantarum 16, 915-925

Mace, M.E., Bell, A.A., Stipanovic, R.D. (1978) Histochemical identification of flavanols in Verticillium wilt-resistant and susceptible cottons. Physiological Plant Pathology 13, 143-149

Mace, M.E., Howell, C.R. (1974) Histochemistry and identification of condensed tannin precursors in the roots of cotton seedlings. Canadian Journal of Botany 52, 2423-2426

Mace, M.E., Solit, E. (1966) Interactions of 3-indolacetic acid and 3-hydroxytyramine in Fusarium wilt of banana. Phytopathology 56, 245-247 
Mace, M.E., Stipanovic, R.D., Bell, A.A. (1990) Relation between sensitivity to terpenoid phytoalexins and virulence to cotton of Verticillium dahliae strains. Pesticide Biochemistry and Physiology 36, 79-82

Mace, M.E., Veech, J.A., Beckman, C.H. (1972) Fusarium wilt of susceptible and resistant tomato isolines: histochemistry of vascular browning. Phytopathology 62, 651-654

Mace, M.E., Wilson, E.M. (1964) Phenol oxidases and their relation to vascular browning in Fusarium-invaded banana roots. Phytopathology 54, 840-842

Maher, E.A., Bate, N.J., Ni, W., Elkind, Y, Dixon, R.A., Lamb, C.J. (1994) Increased disease susceptibility of transgenic tobacco plants with suppressed levels of preformed phenylpropanoid products. Proceedings of the National Academy of Science USA 91, 78027806

Maniatis T, Fritsch EF, Sambrook J (1982) In: Molecular cloning: a laboratory manual. Cold Spring Harbor Laboratory, Cold Spring Harbor.

Mansfield, J.W. (1983) Antimicrobial compounds. In: Biochemical Plant Pathology. Callow, C.A. (ed.) John Wiley \& Sons, Chichester, pp. 237-265

Maor R, Puyesky M, Horwitz BA, Sharon A (1998) Use of green fluorescent protein (GFP) for studying development and fungal-plant interaction in Cochliobolus heterostrophus. Mycological Research 102: 491-496

Matern, U., Grimmig, B., Kneusel, R.E. (1995) Plant cell wall reinforcement in the diseaseresistance response: molecular composition and regulation. Canadian. Journal of Botany 73, S511-S517.

Matsumura, K., Tosa, Y. (1995) The rye mildew fungus carries avirulence genes corresponding to wheat genes for resistance to races of the wheat mildew fungus. Phytopathology 85, 753-756

Matta, A., Gentile, I., Giai, I (1969) Accumulation of phenols in tomato plants infected by different forms of Fusarium oxysporum. Phytopathology 59, 512-513

Mauch-Mani, B., Slusarenko, A.J. (1996) Production of salicylic acid precursors is a major function of phenylalanine ammonia lyase in the resistance of Arabidopsis to Peronospora parasitica. Plant Cell 8, 203-212

McClure, M.E. (1950) Anatomical aspects of the Fusarium wilt of sweet potatoes. Phytopathology 40, 769-775

McLusky, S.R., Bennett, M.H., Beale, M.H., Lewis, M.J., Gaskin, P., Mansfield, J.W. (1999) Cell wall alterations and localized accumulation of feruloyl-3'-methoxytyramine in onion epidermis at sites of attempted penetration by Botrytis allii are associated with actin polarisation, peroxidase activity and suppression of flavonoid biosynthesis. Pant Journal 17, 523-534

McNabb, H.S. Jr, Heybroek, H.M., McDonald, W.L. (1970) Anatomical factors in resistance to Dutch elm disease. Netherland Journal of Plant Pathology 76, 196-204 
Melouk, H. (1992) Verticillium. In: Singleton LL, Mihail JD, Rush CM (eds.) Methods for research on soilborne pathogenic fungi. St Paul, MN, USA: APS Press, 175-178

Mert, M., Kurt, S., Gencer, O., Akiscan, Y., Boyaci, K., Tok, F.M. (2005) Inheritance of resistance to Verticillium wilt (Verticillium dahliae) in cotton (Gossypium hirsutum L.). Plant Breeding 124, 102-104

Mes, J.J., van Doorn, A.A., Wijbrandi, J., Simons, G., Cornelissen, B.J.C., Haring, M.A. (2000) Expression of the Fusarium resistance gene I-2 colocalizes with the site of fungal containment. Plant Journal 23, 183-193

Mol, L., Scholte, K. (1995) Formation of microsclerotia of Verticillium dahliae Kleb. on various plant parts of two potato cultivars. Potato Research 38: 143-150

Mol, L., Scholte, K., Vos, J. (1995) Effects of crop rotation and removal of crop debris on the soil population of two isolates of Verticillium dahliae. Plant Pathology 44, 1070-1074

Mouzeyar, S., Tourvieille De Labrouhe, D., Vear, F. (1993) Histopathological studies of resistance of sunflower (Helianthus annus L.) to downy mildew (Plasmopara halstedii). Journal of Phytopathology 139, 289-297

Müller, W.C., Beckman, C.H. (1974) Ultrastructure of the phenol- storing cells in roots of banana. Physiological Plant Pathology 4, 187-190

Murpy, D.J. 81996) Engineering oil production in rapeseed and other crops. Tibtech 14, 206213

Nachmias, A., Caligari, P.D.S., Brown, J. (1990) Measurement of field-resistance of potatoes to Verticillium wilt (Verticillium dahliae). Potato Research 33, 201-209

Neumann, M.J., Dobinson, K.F. (2003) Sequence tag analysis of gene expression during pathogenic growth and microsclerotia development in the vascular wilt pathogen Verticillium dahliae. Fungal Genetics and Biology 38: 54-62

Nicholson, R.L., Hammerschmidt, R. (1992) Phenolic compounds and their role in disease resistance. Annual Review of Phytopathology 30, 369-389

Okubara, P.A., Paulitz, T.C. 82005) Root defense responses to fungal pathogens: A molecular perspective. Plant and Soil 274, 215-226

Olivain, C., Trouvelot, S., Binet, M.-N., Cordier, C., Pugin, A., Alabouvette, C. (2003) Colonization of flax roots and early physiological responses of flax cells inoculated with pathogenic and nonpathogenic strains of Fusarium oxysporum. Applied and Environmental Microbiology 69, 5453-5462

Olsson, S., Nordbring-Hertz, B. (1985) Microsclerotial germination of Verticillium dahliae as affected by rape rhizosphere. FEMS Microbiological Ecology 31, 293-299

Oren, L., Ezrati, S., Cohen, D., Sharon, A. (2003) Early events in the Fusarium verticillioidesmaize interaction characterized by using a Green Fluorescent Protein-expressing transgenic isolate. Applied and Environmental Microbiology 69:1695-1701 
Otter, T.J. (1996) Untersuchungen zur Ligninbildung bei der Fichte (Picea abies [L.] Karst.). Dissertation Freiburg, pp 45-47

Ouellette, G.B.O., Rioux, D. (1992) Anatomical and physiological aspects of resistance to Dutch elm disease. In: Defense mechanisms of woody plants against fungi. Blanchette, R.A., Biggs, A. (eds.) Springer Verlag, Berlin. Pp. 257-307

Pantou, M.P., Kouvelis, V.N., Typas, M.A. (2006) The complete mitochondrial genome of the vascular wilt fungus Verticillium dahliae: a novel gene order for Verticillium and a diagnostic tool for species identification. Current Genetics 50, 125-136

Pedras, M.S.C., Zheng, Q.-A., Gadagi, R.S., Rimmer, S.R. (2007) Phytoalexins and polar metabolites from the oilseeds canola and rapeseed: Differential metabolic responses to the biotroph Albugo candida and abiotic stress. Phytochemistry, Early Online Article

Pegg, G.F. (1981) Biochemistry and physiology of pathogenesis. In: Mace ME, Bell AA, Beckman CH (eds.) Fungal Wilt Diseases of Plants. Academic Press, New York, 193-253

Pegg, G.F. (1984) The impact of Verticillium diseases in agriculture. Phytopathologia Mediterranea 23: 176-192

Pegg, G.F. (1985) Life in a black hole - the micro-environment of the vascular pathogen. Transactions of the British Mycological Society 85: 1-20

Pegg, G.F. (1974) Verticillium diseases. Review of Plant Pathology 53, 157-182

Pegg, G.F., Brady, B.L. (2002) Verticillium Wilts. Wallingford, UK: CAB Publishing

Pennypacker, B.W., Nelson, P.E. (1972) Histopathology of carnation infected with Fusarium oxysporum f. sp. dianthi. Phytopathology 62: 1318-1326

Portenko, L.G. (2000) Verticillium longisporum - Agent of Verticillium wilt of winter rape in Russia. Mykologiya I Fitopatologiya 34, 52-57

Presley, J.T., Carns, H.R., Taylor, E.E., Schnathorst, W.C. (1966) Movement of conidia of Verticillium albo-atrum in cotton plants. Phytopathology 56, 375

Pritchard, S., Peterson, C., Runion, G.B., Prior, S., Rogers, H. (1997) Atmospheric $\mathrm{CO}_{2}$ concentration, $\mathrm{N}$ availibility, and water status affect patterns of ergastic substance deposition in longleaf pine (Pinus palusitris Mill.) foliage. Trees Structure and Function 118, 494-503

Punt, P.J., Oliver, R., Dingemanse, M.A., Pouwels, P.H., van den Hondel, C.A.M.J.J. (1987) Transformation of Aspergillus based on the Hygromycin B resistance marker from Escherichia coli. Gene 56: 117-124

Radwan, O., Mouzeyar, S., Venisse, J.S., Nicolas, P., Bouzidi, M.F. (2005) Resistance of sunflower to the biotrophic oomycete Plasmopara halstedii is associated with a delayed hypersensitive response within the hypocotyls. Journal of Experimental Botany 56, 26832693

Reichenbach, G.L. (1845) Icones Florae Germanicae et Helveticae, Vol. 7 
Reid, J. (1958) Studies on the fusaria that cause wilt in melon. Canadian Journal of Botany $36,394-410$

Ride, J.P. (1978) The role of cell wall alterations in resistance to fungi. Annals of Applied Biology 89, 302-306

Ride, J.P. (1983) Cell walls and other structural barriers in defense. In: Biochemical Plant Pathology. Callow, J.A. (ed.) Wiley Publishers, Chichester, U.K., pp 215-236

Robb, J., Brisson, J.D., Busch, L., Lu, B.C. (1979) Ultrastructure of wilt syndrome caused by Verticillium dahliae. VII. Correlated light and transmission electron microscope identification of vessel coating and tyloses. Canadian Journal of Botany 57, 822-834

Robb, J., Busch, L.V., Lu, B.C. (1975b) Ultrastructure of wilt syndrome caused by Verticillium dahliae. II. In sunflower leaves. Canadian Journal of Botany 53, 2725-2739

Robb, J., Powell, D.A., Street, P.F.S. (1984) Time-course of wall-coating secretion in Verticillium-infected tomatoes. Physiological and Molecular Plant Pathology 31, 217-226

Robb, J., Powell, D.A., Street, P.F.S. (1989) Vascular coating: a barrier to colonization by the pathogen in Verticillium wilt of tomato. Canadian Journal of Botany 67, 600-607

Robinson, M., Sharon, A. (1999) Transformation of the bioherbicide Colletotrichum gloeosporioides f. sp. aeschynomene by electroporation of germinated conidia. Current Genetics 36: 98-104.

Rodriguez-Galvez, E., Mendgen, K. (1995) Cell wall synthesis in cotton roots after infection with Fusarium oxysporum. Planta 197, 535-545

Rodriguez-Molina, M.C., Medina, I., Torres-Vila, L.M., Cuartero, J. (2003) Vascular colonization patterns in susceptible and resistant tomato cultivars inoculated with Fusarium oxysporum f.sp. lycopersici races 0 and 1. Plant Pathology 52, 199-203

Roncero, M.I.G., Hera, Concepcion, Ruiz-Rubio, M., Garcia Maceira, F.I., Madrid, M.P., Caracuel, Z., Calero, F., Delgado-Jarana, J., Roldán-Rodríguez, R., Martínez-Rocha, A.L., Velasco, C., Roa, J., Martín-Urdiroz, M., Córdoba, D., Die Pietro, A. (2003) Fusarium as a model for studying virulence in soilborne plant pathogens. Physiological and Molecular Plant Pathology 62, 87-89

Ryals, J.A., Neuenschwander, U.H., Willits, M.G., Molina, A., Steiner, H.-Y. (1996) Systemic Acquired Resistance. Plant Cell 8, 1809-1819

Rygulla, W., Snowdon, R.J., Eynck, C., Koopmann, B., von Tiedemann, A., Lühs, W., Friedt, W. (2007a) Broadening the genetic basis of Verticillium longisporum resistance in Brassica napus by interspecific hybridisation. Plant Breeding, Online Early Article

Rygulla, W., Seyis, F., Lühs, W., Eynck, C., von Tiedemann, A., Friedt, W, Snowdon, R.J. (2007b) Combination of resistance to Verticillium longisporum from zero erucic acid Brassica oleracea and oilseed Brassica rapa genotypes in resynthesized rapeseed (Brassica napus) lines. Phytopathology 97 (11), 1391-1396 
Salerno, M.I., Gianinazzi, S., Gianinazzi-Pearson, V. (2000) Effects on growth and comparison of root tissue colonization patterns of Eucalyptus viminalis by pathogenic and nonpathogenic strains of Fusarium oxysporum. New Phytologist 146, 317-324

Sarkanen, K.V., Hergert, H.L. (1971) Classification and distribution. In: Lignins: Occurrence, Formation, Structure, and Reactions. Sarkanen, K.V., Ludwig, C.H. (eds.) Wiley-Interscience,

Schaible, L., Cannon, O.S., Waddoups, V. (1951) Inheritance of resistance to Verticillium wilt in a tomato cross. Phytopathology 41, 986-990

Scheffer, R.P., Walker, J.C. (1953) The physiology of Fusarium wilt of tomato. Phytopathology 43, 116-125

Schmutz, A., Jenny, T., Amrhein, N., Ryser, U. (1993) Caffeic acid and glycerol are constituents of the suberin layers in green cotton fibres. Planta 189, 453-460

Schnathorst WC (1981) Life cycle and epidemiology of Verticillium. In: Mace ME, Bell AA, Beckmann CH (eds.) Fungal Wilt Diseases of Plants. Academic Press, New York, 81-111

Schöber, B. (1971) Physiologische Veränderungen in der Kartoffelknolle nach Verletzung und Infektion mit Phytophthora infestans. Potato Research 14, 39-48

Schreiber, L.R., Green, R.J.Jr (1963) Effects of root exudates on germination of conidia and microsclerotia of Verticillium albo-atrum inhibited by the soil fungistatic principle. Phytopathology 53, 260-264

Shan, X.C., Goodwin, P.H. (2004) Monitoring host nuclear migration and degradation with green fluorescent protein during compatible and incompatible interactions of Nicotiana tabacum with Colletotrichum species. Journal of Phytopathology 152: 454-560

Shiraishi, T., Yamaoka, N., Kunoh, H., 1989. Association between increased phenylalanine ammonia-lyase activity and cinnamic synthesis and the induction of temporary inaccessibility caused by Erysiphe graminis primary germ tube penetration of the barley leaf. Physiological and Molecular Plant Pathology 34, 75-83.

Shuen, S.K., Buswell, J.A. (1992) Effect of lignin derived phenols and their methylated derivatives on the growth of Lentinus spp. Letters in Applied Micobiology 15, 12-14

Simko, I., Costanzo, S., Haynes, K.G., Christ, B.J., Jones, R.W. (2004a) Linkage disequilibrium mapping of a Verticillium dahliae resistance quantitative trait locus in tetraploid potato (Solanum tuberosum) through a candidate gene approach. Theoretical and Applied Genetics 108, 217-224

Simko, I., Haynes, K.G., Ewing, E.E., Costanzo, S., Christ, B.J., Jones, R.W. (2004c) Mapping genes for resistance to Verticillium albo-atrum in tetraploid and diploid potato populations using haplotype association tests and genetic linkage analysis. Molecular Genetics and Genomics 271, 522-531

Singleton, V.L., Orthofer, R, Lamuela-Raventós, R.M. (1999) Analysis of total phenols and other oxidation substrates and antioxidants by means of Folin-Ciocalteu reagent. Methods in Enzymology 299, 152-178 
Sinha, A.K., Wood, R.K.S. (1968) Studies on the nature of resistance in tomato plants to Verticillium albo-atrum. Annals of Applied Biology 59, 143-154

Siranidou, E., Kang, Z., Buchenauer, H. (2002) Studies on symptom development, phenolic compounds and morphological defence responses in wheat cultivars differing in resistance to Fusarium head blight. Journal of Phytopathology 150, 200-208

Smith, D.N., King, W.J., Tooper, C.P., Boma, F., Cooper, J.F. (1995a) Alternative techniques for the application of sulphur dust to cashew trees for the control of powdery mildew caused by the fungus Oidium anacardii in Tanzania. Crop Protection 14, 555-560

Smith, R., Walker, J.C. (1930) A cytological study of cabbage plants in strains susceptible or resistant to yellows. Journal of Agricultural Research 41, 17-35

Snook, M.E., Csions, S., Chortyk, O.T. (1992) Inhibition of growth of Phytophthora parasitica var. nicotianae by aromatic acids and coumarins in a laboratory bioassay. Journal of Chemical Ecology 18, 1287-1297

Söchting, H.P., Verreet, J.-A. (2004) Effects of different cultivation systems (soil management, nitrogen fertilization) on the epidemics of fungal diseases in oilseed rape (Brassica napus L. var. napus). Journal of Plant Diseases and Protection 111: 1-29

Solla, A., Gil, L. (2002) Xylem vessel diameter as a factor in resistance of Ulmus minor to Ophiostoma novo-ulmi. Forest Pathology 32, 123-134

Spellig, T., Bottin, A. Kahmann, R. (1996) Green fluorescent protein (GFP) as a new vital marker in the phytopathogenic fungus Ustilago maydis. Molecular and General Genetics 252: 503-509

Sprague, S.J., Balesdent, M.H., Brun, H., Hayden, H.L., Marcroft, S.J., Pinochet, X., Rouxel, T., Howlett, B.J. (2006) Major gene resistance in Brassica napus (oilseed rape) is overcome by changes in virulence of populations of Leptosphaeria maculans in France and Australia. European Journal of Plant Pathology 114, 33-40

Stark, C. (1961) Das Auftreten der Verticillium-Tracheomykosen in Hamburger GartenbauKulturen. Gartenbauwissenschaft 26: 493-528

Statistisches Bundesamt (2007) Statistik kurz gefasst: Anbauflächen der Europäischen Union 2007

Steinbach, P., Kreye, H., Wolf, G.A. (2005) Bundesweites „Verticillium-Monitoring“. Bewertung von Diagnosesicherheit und Risikofaktoren. Raps 23, 20-26

Steventon, L.A., Okori, P., Dixelius, C. (2001) An investigation of the susceptibility of Arabidopsis thaliana to isolates of two species of Verticillium. Journal of Phytopathology $149,395-401$

Steventon, L.A., Fahleson, J., Hu, Q., Dixelius, C. (2002a) Identification of the causal agent of Verticillium wilt of winter oilseed rape in Sweden, Verticillium longisporum. Mycological Research 106: 570-578 
Steventon, L.A., Happstadius, I., Okori, P., Dixelius, C. (2002b) Development of a rapid technique for the evaluation of the response of Brassica napus to Verticillium wilt. Plant Disease $86,854-858$

Strack, D., Heilemann, J., Mömken, M., Wray, V. (1988) Cell wall-conjugated phenolics from coniferae leaves. Phytochemistry 27: 3517-3521

Street, P.F.S., Robb, J., Ellis, B.E. (1986) Secretion of vascular coating components by xylem parenchyma cell of tomatoes infected with Verticillium albo-atrum. Protoplasma 132, 1-11

Subbarao, K.V., Chassot, A., Gordon, T.R., Hubbard, J.C., Bonello, P., Mulin, R., Okamoto, D., Davis, R.M., Koike, S.T. (1995) Genetic relationships and cross pathogenicities of Verticillium dahliae isolates from cauliflower and other crops. Phytopathology 85, 1105-1112

Sung, G.-H., Spatafora, J.W., Zare, R., Hodge, K.T., Gams, W. (2001) A revision of Verticillium sect. Prostrata. II. Phylogenetic analyses of SSU and LSU nuclear rDNA sequences from anamorphs and teleomorphs of the Clavicipitaceae. Nova Hedwigia 72,311328

Svenson, C.H., Lerenius, C. (1987) An investigation on the effect of Verticillium wilt (Verticillium dahliae Kleb.) on oilseed rape. Working group integrated control in oilseed rape. IOBC/WPRS Bulletin X/4: 30-34

Swain, T., Hillis, W.E. (1959) The phenolic constituents of Prunus domestica I. The quantitative analysis of phenolic constituents. J. Sci. Food Agric. 10, 63-68

Talboys, P. W. (1957) The possible significance of toxic metabolites of Verticillium alboatrum in the development of hop wilt symptoms. Transactions of the British Mycological Society 40, 415-427

Talboys, P.W. (1958a) Degradation of cellulose by Verticillium albo-atrum. Transactions of the British Mycological Society 41, 242-248

Talboys, P.W. (1958b) Association of tylosis and hyperplasia of the xylem with vascular invasion of the hop by Verticillium albo-atrum. Transactions of the British Mycological Society 41, 249-260

Talboys, P.W. (1964) A concept of the host-parasite realtionship in Verticillium wilt diseases. Nature 202, 361-362

Talboys, P.W. (1968) Water deficits in vascular disease. In: Kozlowski, T.T. (ed.) Water Deficits and Plant Growth, Vol. 2. Academic Press Inc, New York, pp. 255-311

Talboys, P.W. (1984) Damage symptoms and crop loss caused by vascular pathogens. In: Plant Diseases: Infection, Damage, and Loss. Wood, R.K.S., Jellis, G.J. (eds.) Blackwell Scientific Publications, Oxford. pp. 171-187

Taylor, A.O., Zucker, M. (1966) Turnover and metabolism of chlorogenic acid in Xanthium leaves. Plant Physiology 41, 1350-1359 
Thordal-Christensen, H. (2003) Fresh insights into processes of nonhost resistance. Current Opinion in Plant Biology 6, 351-357

Threlfall, R.J. (1959) Physiological studies on the Verticillium wilt disease of tomato. Annals of Applied Biology 47, 57-77

Tian, S., Wolf, G.A., Weinert, J. 2005. Accurate assesment of wheat and triticale cultivar resistance to Septoria tritici and Stagonospora nodorum infection by Biotin/Avidin ELISA. Plant Disease 89, 1229-1234

Tjamos, E.C., Smith, I.M. (1974) The role of phytoalexins in the resistance of tomato to Verticillium wilt. Physiological Plant Pathology 4, 249-260

Tjamos, E.C., Smith, I.M. (1975) The expression of resistance to Verticillium albo-atrum in monogenically resistant tomato varieties. Physiological Plant Pathology 6, 215-225

Tsien, R.Y. (1998) The green fluorescent protein. Annual Review of Biochemistry 67, 509544

Tuncel, G., Nergiz, C. (1993) Antimicrobial effect of some olive phenols in a laboratory medium. Letters in Applied Microbiology 17, 300-302

Valette, C., Andary, C., Geiger, J.P., Sarah, J.L., Nicole, M. (1998) Histochemical and cytochemical investigations of phenols in roots of banana infected by the burrowing nematode Radopholus similis. Phytopathology 88, 1141-1148

Van Alfen NK (1989) Molecular bases for virulence and avirulence of fungal wilt pathogens. In: Tjamos EC, Beckman CH (eds.) Vascular Wilt Diseases of Plants. Springer, Berlin, 19-32

Van den Berg, N., Berger, D.K., Hein, I., Birch, P.R.J., Wingfield, M.J., Viljoen, A. (2007) Tolerance in banana to Fusarium wilt is associated with early up-regulation of cell wallstrengthening genes in the roots. Molecular Plant Pathology 8, 333-341

Van den Berg, N., Crampton, B.G., Hein, I., Birch, P.R.J., Berger, D.K. (2004) High throughput screening of suppression subtractive hybridization cDNA libraries using DNA microarray analysis. Biotechniques $37,818-824$

Vance, C.P., Kirk, T.K., Sherwood, R.T. (1980) Lignification as a mechanism of disease resistance. Annual Reviews of Phytopathology 18, 259-288

VanderMolen, G.E., Beckman, C.H., Rodehorst, E. (1987) The ultrastructure of tylose formation in resistant banana following inoculation with Fusarium oxysporum f. sp. cubense. Physiological and Molecular Plant Pathology 31, 185-200

Veronese, P., Narasimham, M.L., Stevenson, R.A. (2003) Identification of a locus controlling Verticillium disease symptom response in Arabidopsis thaliana. Plant Journal 35, 574-587

Waggoner, P.E., Dimond, A.E. (1956) Polyphenol oxidases and substrates in potato and tomato stems. Phytopathology 46, 495-497 
Walker, J.C. (1963) The physiology of disease resistance. W. Va. Agr. Expt. Sta. Bull. 488T, $1-25$

Walker, J.C. (1965) Use of environmental factors in screening for disease resistance. Annual Reviews of Phytopathology 3, 197-208

Walker, J.C., Smith, R. (1930) Effect of environmental factors upon the resistance of cabbage to yellows. Journal of Agricultural Research 41, 1-15

Walsh, J.A., Sharpe, A.G., Jenner, C.E., Lydiate, D.J. (1999) Characterisation of resistance to turnip mosaic virus in oilsed rape (Brassica napus) and genetic mapping of TuRB01. Theoretical and Applied Genetics 99, 1149-1154

Wardrop, A.B. (1971) Occurrence and formation in plants. In: Lignins: Occurrence, Formation, Structure, and Reactions. Sarkanen, K.V., Ludwig, C.H. (eds.) Wiley Interscience, New York, pp. 19-41

Warkentin, T.D., Rashid, K.Y., Xue, A.G. (1996) Fungicidal control of powdery mildew in field pea. Canadian Journal of Plant Science 76, 933-935

Weisshaar, B, Jenkins, G.I. (1998) Phenylpropanoid biosynthesis and its regulation. Current Opinions in Plant Biology 1, 251-257

Wen-Jun, S., Forde, B.G. (1989) Efficient transformation of Agrobacterium spp. by high voltage electroporation. Nucleic Acid Research 17, 8385

Whetten, R., Sederoff, R. (1995) Lignin biosynthesis. Plant Cell 7, 1001-1013

Whetten, R.W., MacKay, J.J., Sederoff, R.R. (1998) Recent advances in understanding lignin biosynthesis. Annual Review of Plant Physiology and Plant Molecular Biology 49, 585-609

Whitmore, F.W. (1978a) Lignin-protein complex catalyzed by peroxidase. Plant Science Letters 13, 241-245

Whitmore, F.W. (1978b) Lignin-carbohydrate complex formed in isolated cell walls of callus. Phytochemistry 17, 421-425

Wilhelm, S., Sagen, J.E., Tietz, H (1970) Seabrook Sea Island (Gossypium barbadense) x Rex (Gossypium hirsutum) crosses give Verticillium wilt-resistant, upland-type all fertile offspring. In: Proc. Beltwide Cooton Prod. Res. Conf. Houston, Texas, pp 70-76

Wilhelm, S., Sagen, J.E., Tietz, H (1974) Resistance to Verticillium wilt in cotton: Sources, techniques of identification, inheritance trends and the resistance of potential multiline cultivars. Phytopathology 64, 924-931

Williams, J., Hall, S.A., Hawkesford, M.J., Beale, M.H., Cooper, R.M. (2002) Elemental sulfur and thiol accumulation in tomato and defense against a fungal vascular pathogen. Plant Physiology 128, 150-159 
Williams, J.S., Cooper, R.M. (2003) Elemental sulphur is produced by diverse plant families as a component of defence against fungal and bacterial pathogens. Physiological and Molecular Plant Pathology 63, 3-16

Williams, J., Cooper, R.M. (2004) The oldest fungicide and newest phytoalexin - a reappraisal of the fungitoxicity of elemental sulphur. Plant Pathology 53, 263-279

Wink, M. (1997) Compartmentation of secondary metabolites and xenobiotics in plant vacuoles. Advances in Botanical Research 25, 141-169

Wolf, G.A., Weinert, J. (2003) Problemschläge früh erkennen. DLG-Mitteilungen 7, 48-50

Wolter, K.E., Harkin, J.M., Kirk, T. (1974) Guaiacyl lignin associated with vessels in aspen callus cultures. Physiol. Plant. 31, 140-143

Wood, J.R., Vivian, A., Jenner, C., Mansfield, J.W., Taylor, J.D. (1994) Detection of a gene in pea controlling nonhost resistance to Pseudomonas syringae pv. phaseolicola. Molecular Plant-Microbe Interactions 7, 534-537

Wood, R.K.S. (1961) Verticillium wilt of tomatoes and the role of pectic and cellulolytic enzymes. Annals of Applied Biology 49, 120-139

Woodward, S., Pearce, R.B. (1988) The role of stilbenes in resistance of Sitka spruce (Picea sitchensis (Bong.) Carr.) to entry of fungal pathogens. Physiological and Molecular Plant Pathology 33, 127-149

Xiao, C.L., Subbarao, K.V. (2000) Effects of irrigation and Verticillium dahliae on cauliflower root and shoot growth dynamics. Phytopathology 90: 995-1004

Young, J.E., Zhao, X., Carey, E.E., Welti, R., Yang, S, Wang, W. (2005) Phytochemical phenolics in organically grown vegetables. Molecular Nutrition \& Food Research 49, 11361142

Yui, S., Ashizawa, M., Yamagishi, H. (1985) New soilborne disease of Chinese cabbage caused by Verticillium dahliae Klebahn, and the resistance in Brassica campestris. Cruciferae newsletter 10, 106-107

Zare, R., Gams, W., Culham, A. (2000) A revision of Verticillium sect. Prostata I. Phylogenetic studies using ITS sequences. Nova Hedwigia 71:465-480

Zare, R., Gams, W., Schroers, H.-J. (2004) The type species of Verticillium is not congeneric with the plant-pathogenic species placed in Verticillium and it is not the anamorph of 'Nectria' inventa. Mycol Res 108:576-582

Zeise, K. (1992) Gewächshaustest zur Resistenzprüfung von Winterraps (Brassica napus L. var. oleifera Metzger) gegen den Erreger der Rapswelke Verticillium dahliae Kleb. Nachrichtenblatt Deutscher Pflanzenschutzdienst 44: 125-128

Zeise, K., Seidel, D. (1990) Zur Entwicklung und Schadwirkung der VerticilliumWelkekrankheit am Winterraps. Raps 8: 20-22 
Zeise, K., von Tiedemann, A. (2001) Morphological and physiological differentiation among vegetative compatibility groups of Verticillium dahliae in relation to $V$. longisporum. Journal of Phytopathology 149: 469-475

Zeise, K., von Tiedemann, A. (2002a) Host specialization among vegetative compatibility groups of Verticillium dahliae in relation to Verticillium longisporum. Journal of Phytopathology 150: 112-119

Zeise, K., von Tiedemann, A. (2002b) Application of RAPD-PCR for virulence type analysis within Verticillium dahliae and Verticillium longisporum. Journal of Phytopathology 150: 557-563

Zeise, K., Seidel, D. (1990) Zur Entwicklung und Schadwirkung der VerticilliumWelkekrankheit am Winterraps. Raps 8: 20-22

Zeise, K., Steinbach, P. (2004) Schwarze Rapswurzeln und der Vormarsch der VerticilliumRapswelke. Raps 2004 (4) 170-174

Zeyen, R.J., Bushnell, W.R., Carver, T.L., Robbins, M.P., Clark, T.A., Boyles, D.A., Vance, C.P. (1995) Inhibiting phenylalanine ammonia lyase and cinnamyl-alcohol dehydrogenase suppresses MLa1 (HR) but not mLo5 (non-HR) barley powdery mildew resistances. Physiological and Molecular Plant Pathology 47, 119-140

Zhou, L., Hu, Q., Johannson, A., Dixelius, C. (2006) Verticillium longisporum and Verticillium dahliae: infection and disease in Brassica napus. Plant Pathology 55: 137-144

Zielenski, D., Sadowski, C. (1995) A preliminary study on Verticillium dahliae Kleb. in winter oilseed rape in Poland. In: Murphy D (ed.). Proceedings of the 9th International Rapeseed Conference, Cambridge. 4-7 July 1995. GciRC, Cambridge, UK, 649-651

Zinkernagel, V. (1982) Zur Entwicklung von Verticillium spp. in anfälligen und toleranten Hopfensorten nach natürlicher und künstlicher Infektion. Zeitschrift für Pflanzenkrankheiten und Pflanzenschutz 89, 205-218

Zou WJ, Yoneyama K, Takeuchi Y, Iso S, Rugmekarat S, Chae SH, Sato D, Joel DM (2004) In vitro infection of host roots by differentiated calli of the parasitic plant Orobranche. Journal of Experimental Botany 55: 899-907 


\section{Appendix}

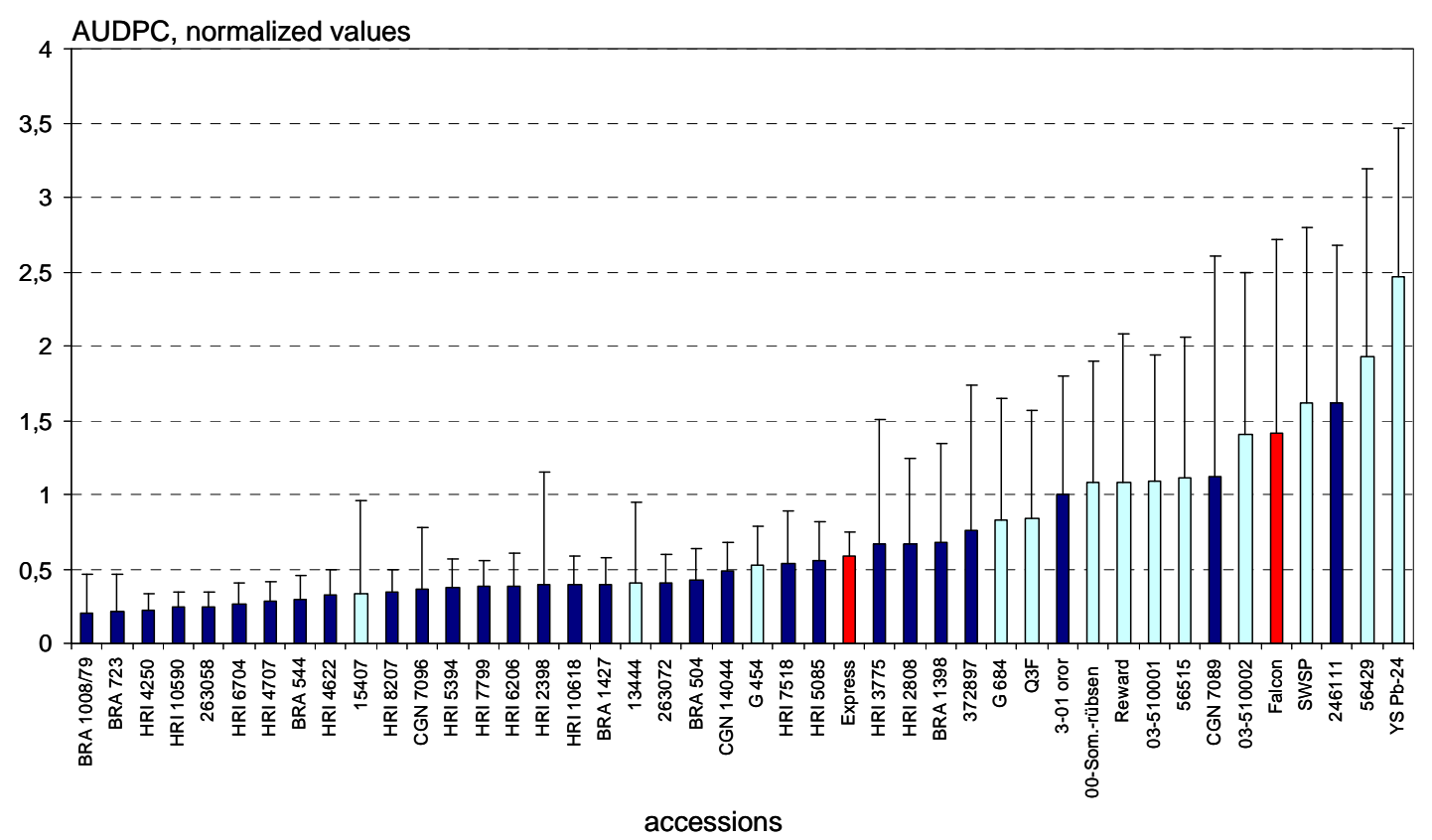

Fig. A 1. Verticillium longisporum resistance response of Brassica rapa (light blue), B. oleracea (dark blue) accessions and reference rapeseed cultivars 'Express' and 'Falcon' (red) measured by area under the disease progress curve (AUDPC) from 23.04.2004 to 13.05.2004. Columns and whiskers represent mean values and standard errors from 20 infected plants of each accession. AUDPC value of Express represents that of the second screening run.

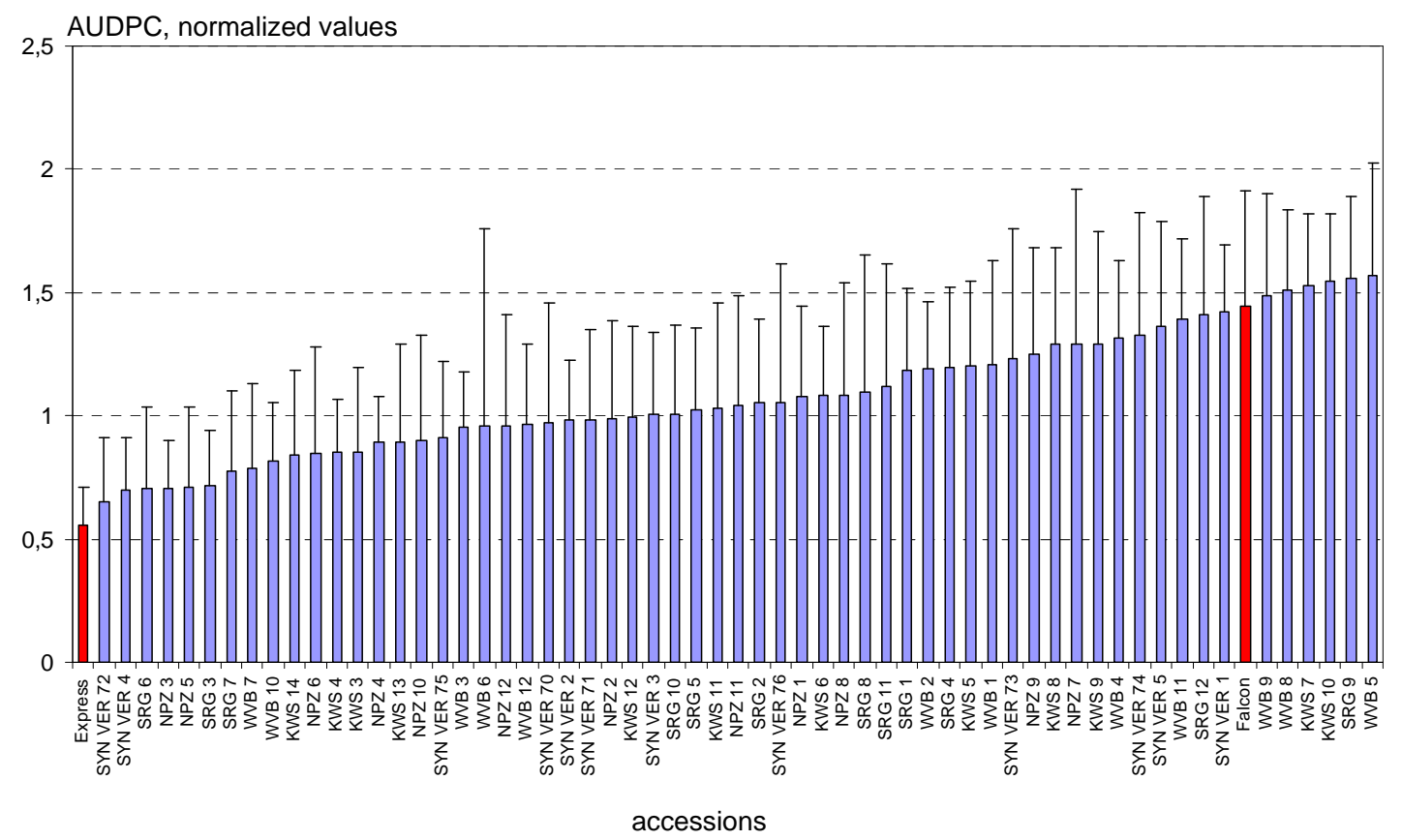

Fig. A 2. Verticillium longisporum resistance response of Brassica napus (purple) accessions and reference rapeseed cultivars 'Express' and 'Falcon' (red) measured by area under the disease progress curve (AUDPC) from 01.06.2004 to 22.06.2004. Columns and whiskers represent mean values and standard errors from 20 infected plants of each accession. 


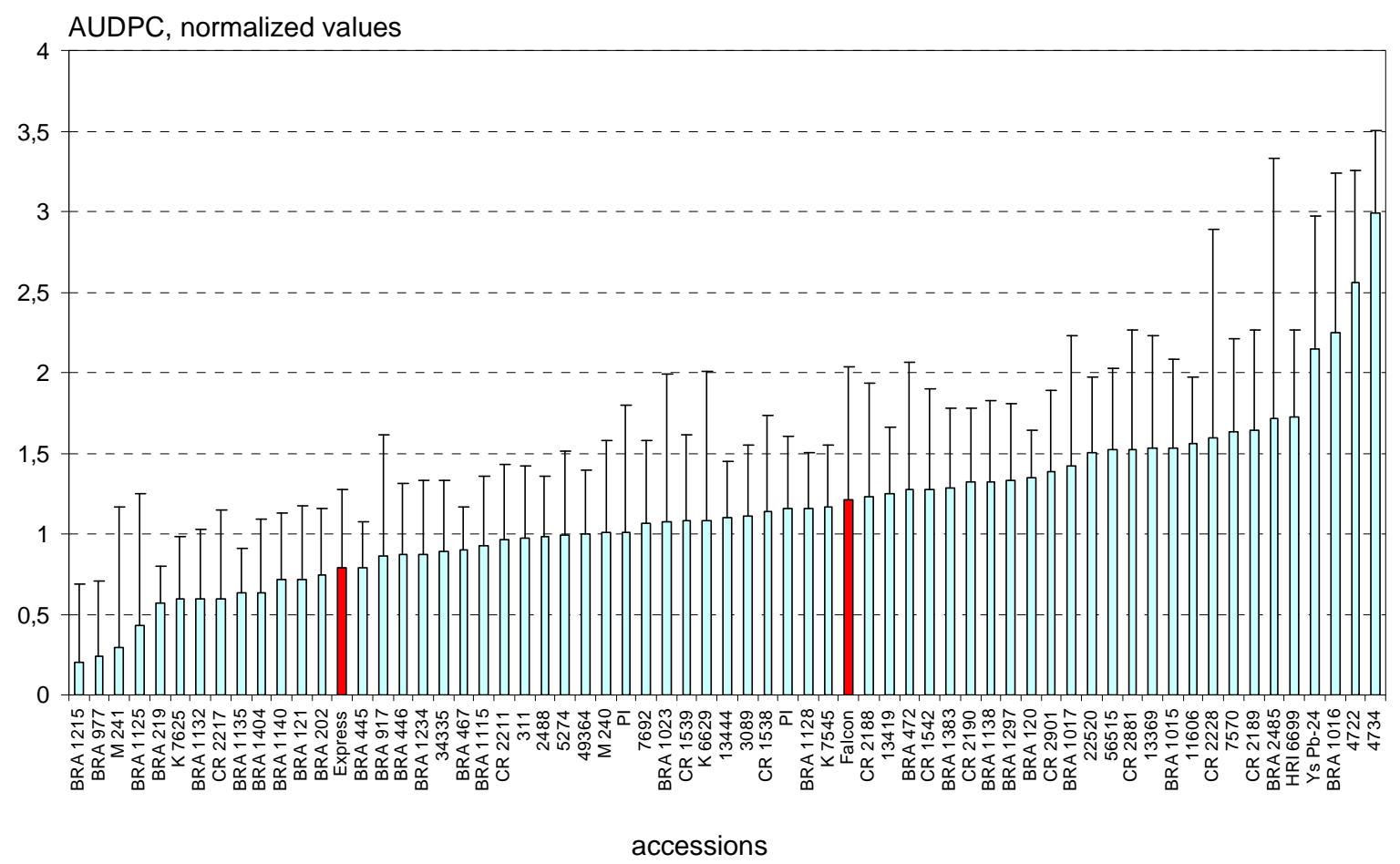

Fig. A 3. Verticillium longisporum resistance response of Brassica rapa (light blue) accessions and reference rapeseed cultivars 'Express' and 'Falcon' (red) measured by area under the disease progress curve (AUDPC) from 12.07.2004 to 02.08.2004. Columns and whiskers represent mean values and standard errors from 20 infected plants of each accession.

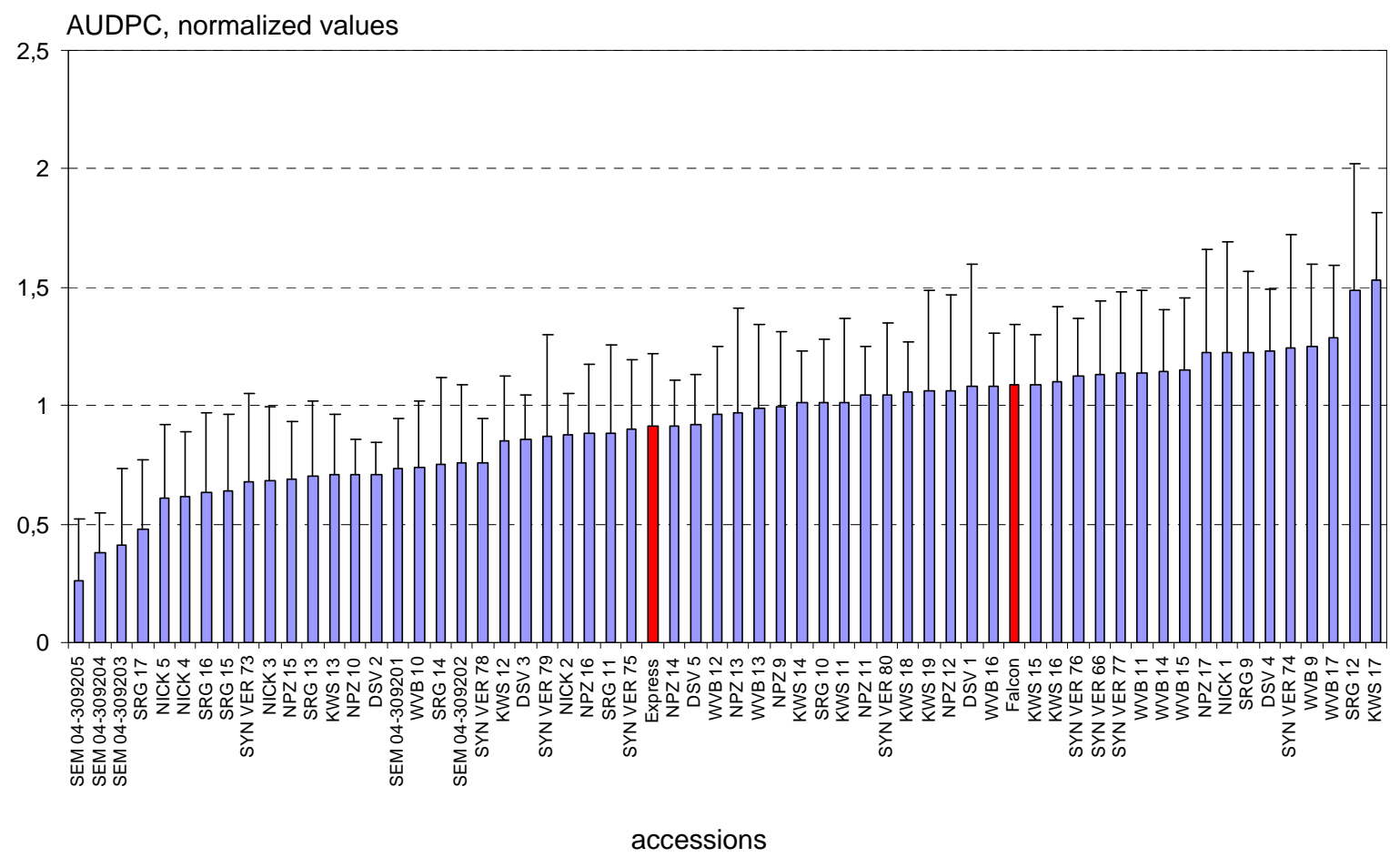

Fig. A 4. Verticillium longisporum resistance response of Brassica napus (purple) accessions and reference rapeseed cultivars 'Express' and 'Falcon' (red) measured by area under the disease progress curve (AUDPC) from 11.08.2004 to 01.09.2004. Columns and whiskers represent mean values and standard errors from 20 infected plants of each accession. 


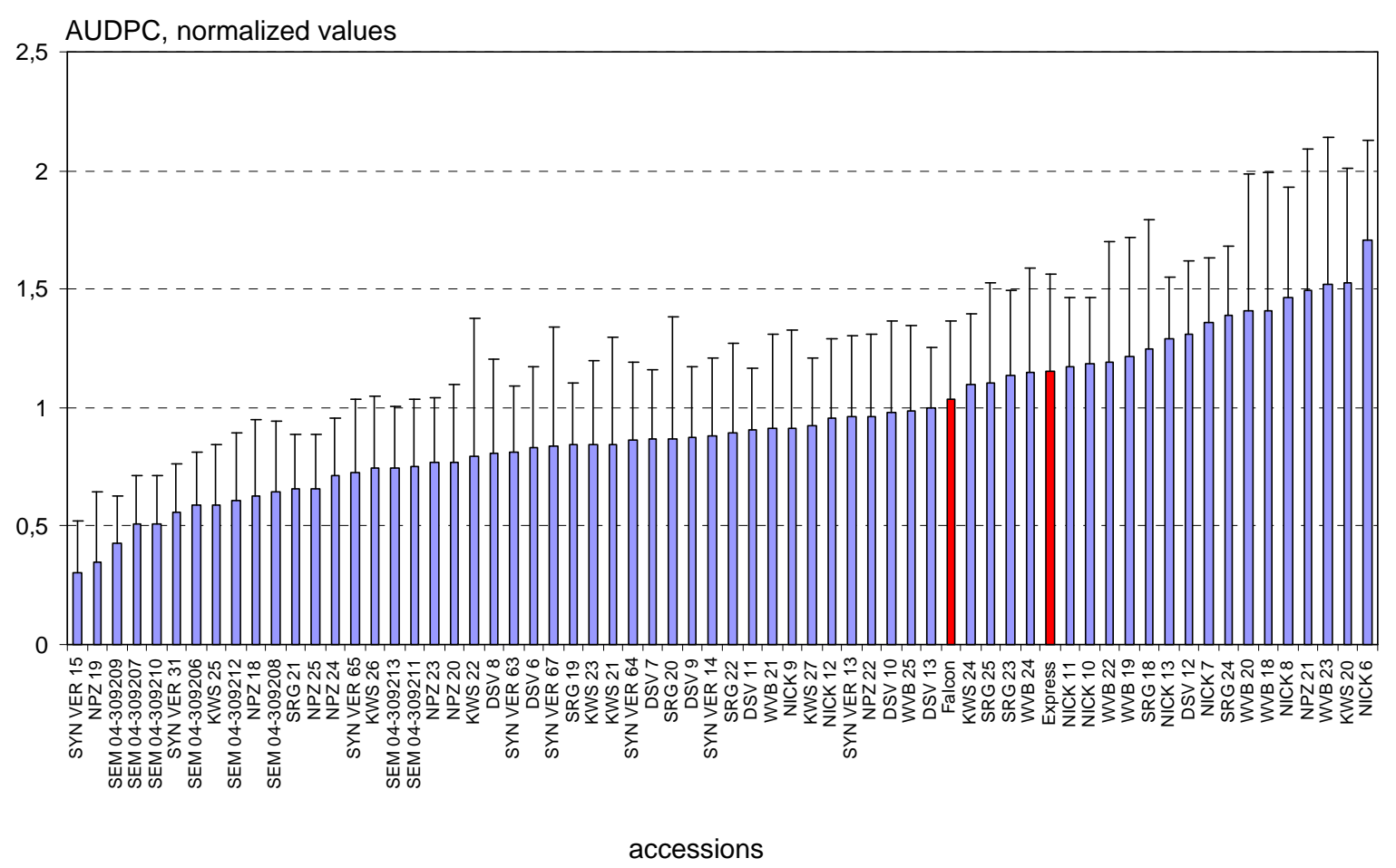

Fig. A 5. Verticillium longisporum resistance response of Brassica napus (purple) accessions and reference rapeseed cultivars 'Express' and 'Falcon' (red) measured by area under the disease progress curve (AUDPC) from 14.09.2004 to 05.10.2004. Columns and whiskers represent mean values and standard errors from 20 infected plants of each accession.

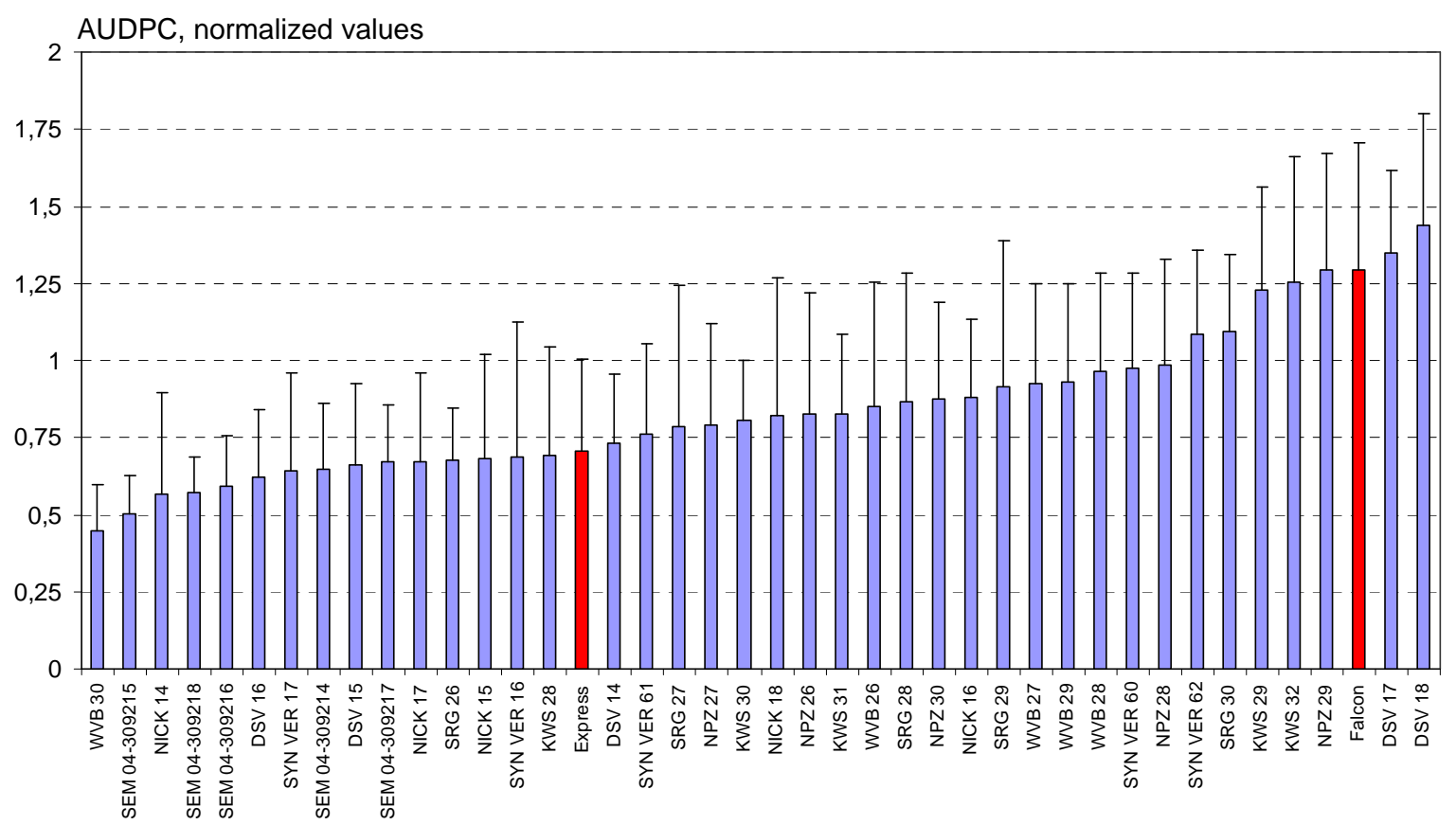

accessions

Fig. A 6. Verticillium longisporum resistance response of Brassica napus (purple) accessions and reference rapeseed cultivars 'Express' and 'Falcon' (red) measured by area under the disease progress curve (AUDPC) from 10.11.2004 to 01.12.2004. Columns and whiskers represent mean values and standard errors from 20 infected plants of each accession. 


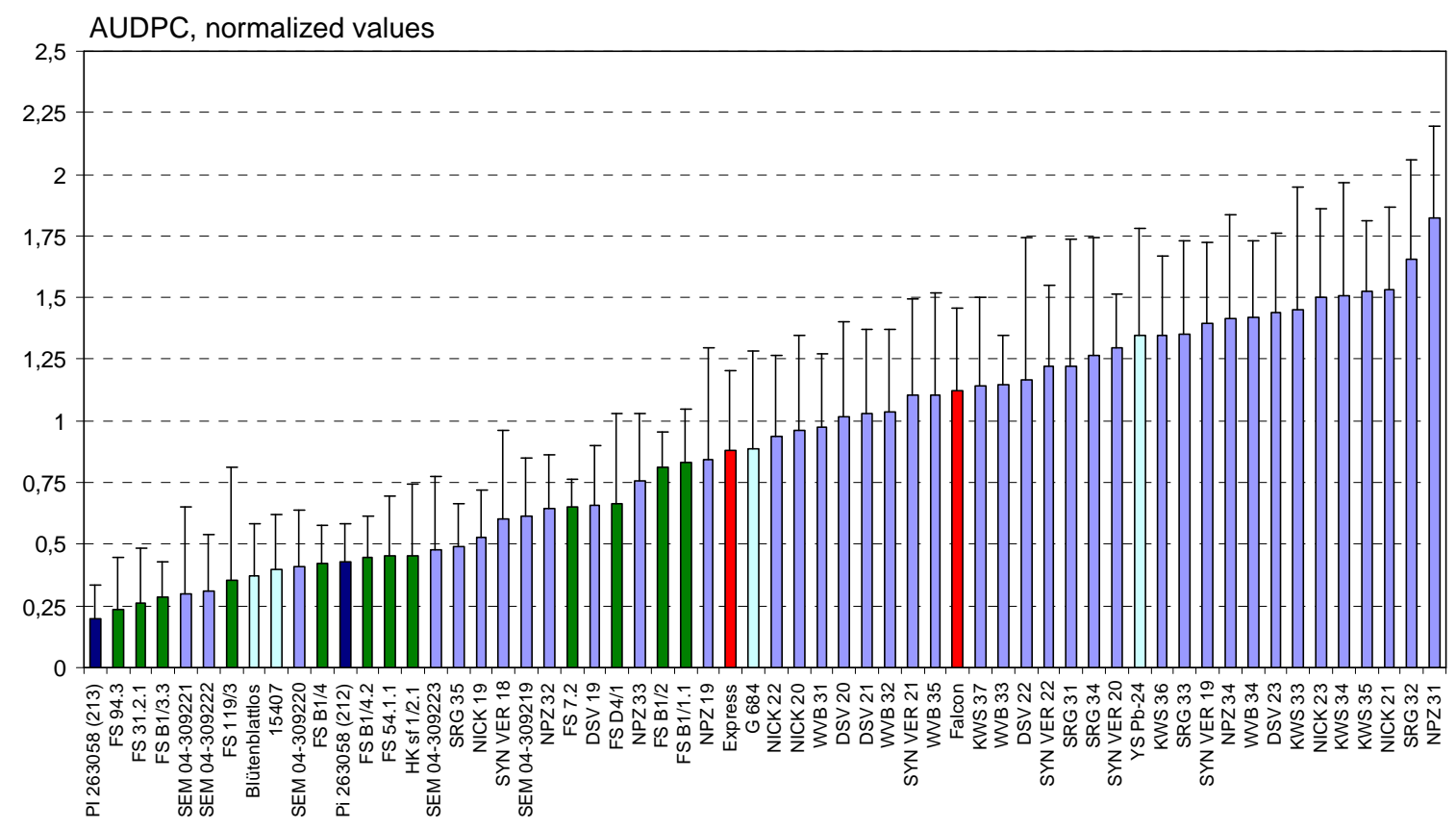

accessions

Fig. A 7. Fig. A 6. Verticillium longisporum resistance response of Brassica rapa (light blue), B. oleracea (dark blue), B. napus (purple) accessions, resynthesized rape seed lines (green) and reference rapeseed cultivars 'Express' and 'Falcon' (red) measured by area under the disease progress curve (AUDPC) from 12.01.2005 to 02.02.2005. Columns and whiskers represent mean values and standard errors from 20 infected plants of each accession.

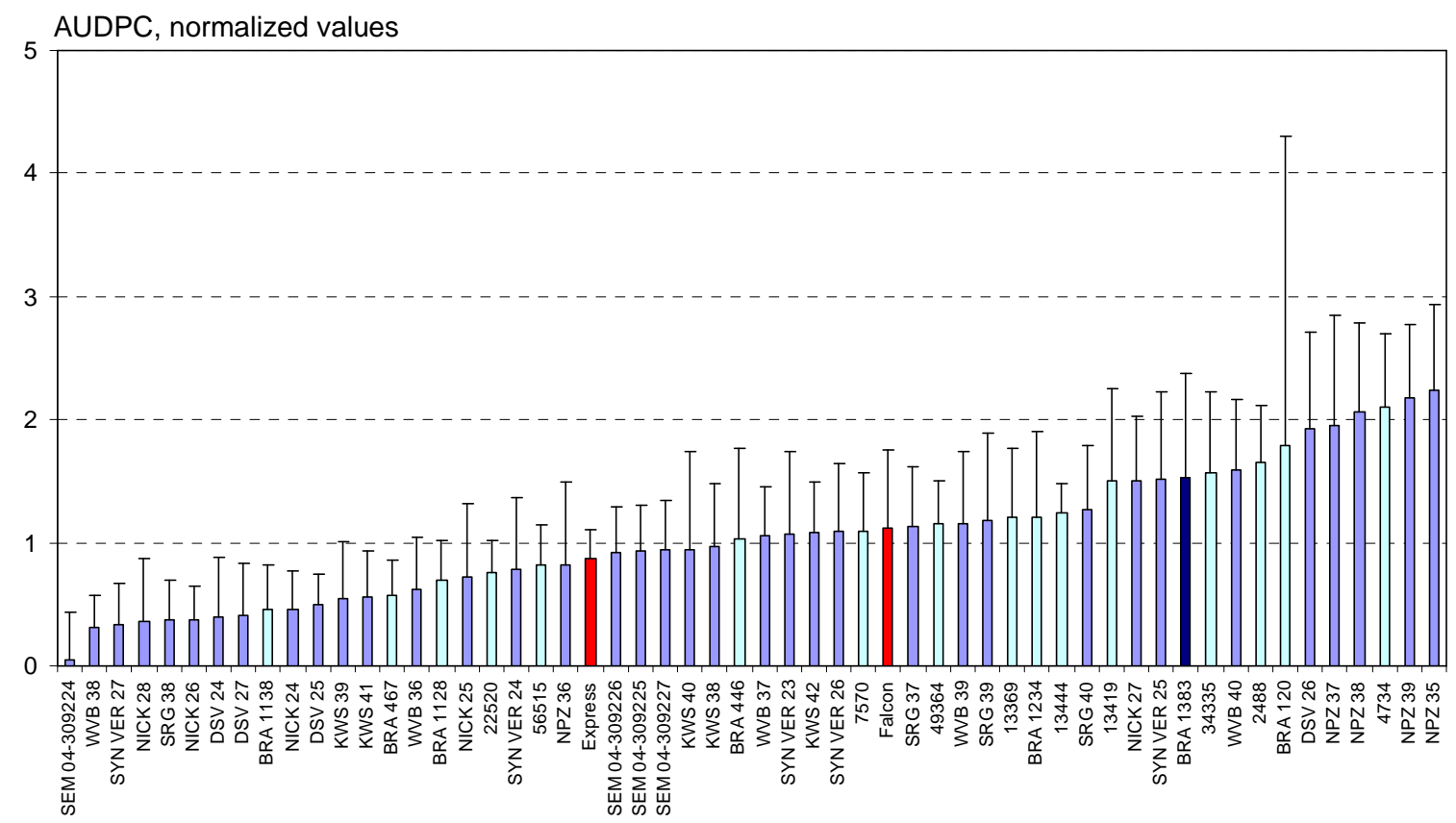

accessions

Fig. A 8. Verticillium longisporum resistance response of Brassica rapa (light blue), B. oleracea (dark blue), B. napus (purple) accessions, and reference rapeseed cultivars 'Express' and 'Falcon' (red) measured by area under the disease progress curve (AUDPC) from 15.02.2005 to 08.03.2005. Columns and whiskers represent mean values and standard errors from 20 infected plants of each accession. 


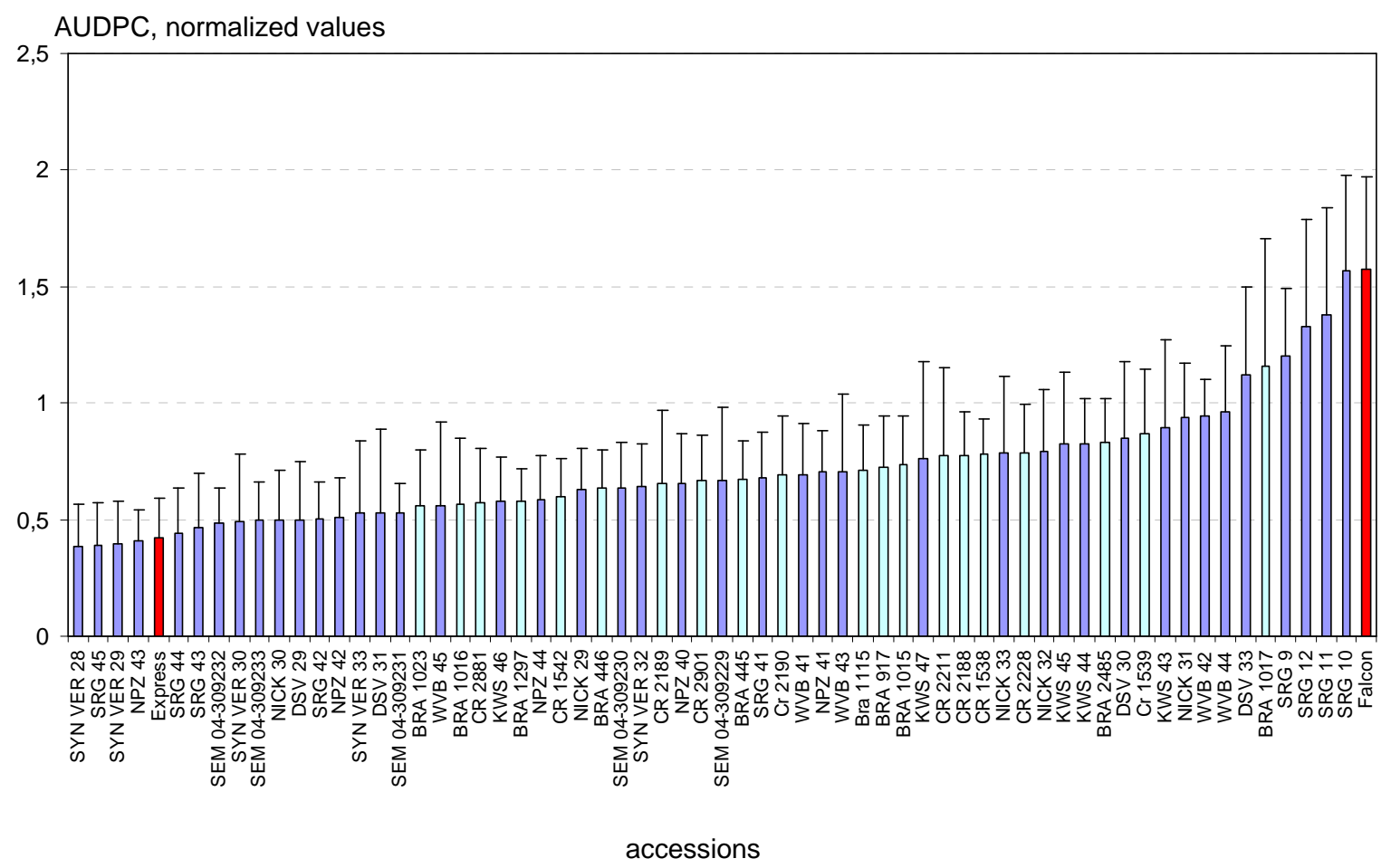

Fig. A 9. Verticillium longisporum resistance response of Brassica rapa (light blue), B. napus (purple) accessions, and reference rapeseed cultivars 'Express` and 'Falcon' (red) measured by area under the disease progress curve (AUDPC) from 12.04.2005 to 03.05.2005. Columns and whiskers represent mean values and standard errors from 20 infected plants of each accession.

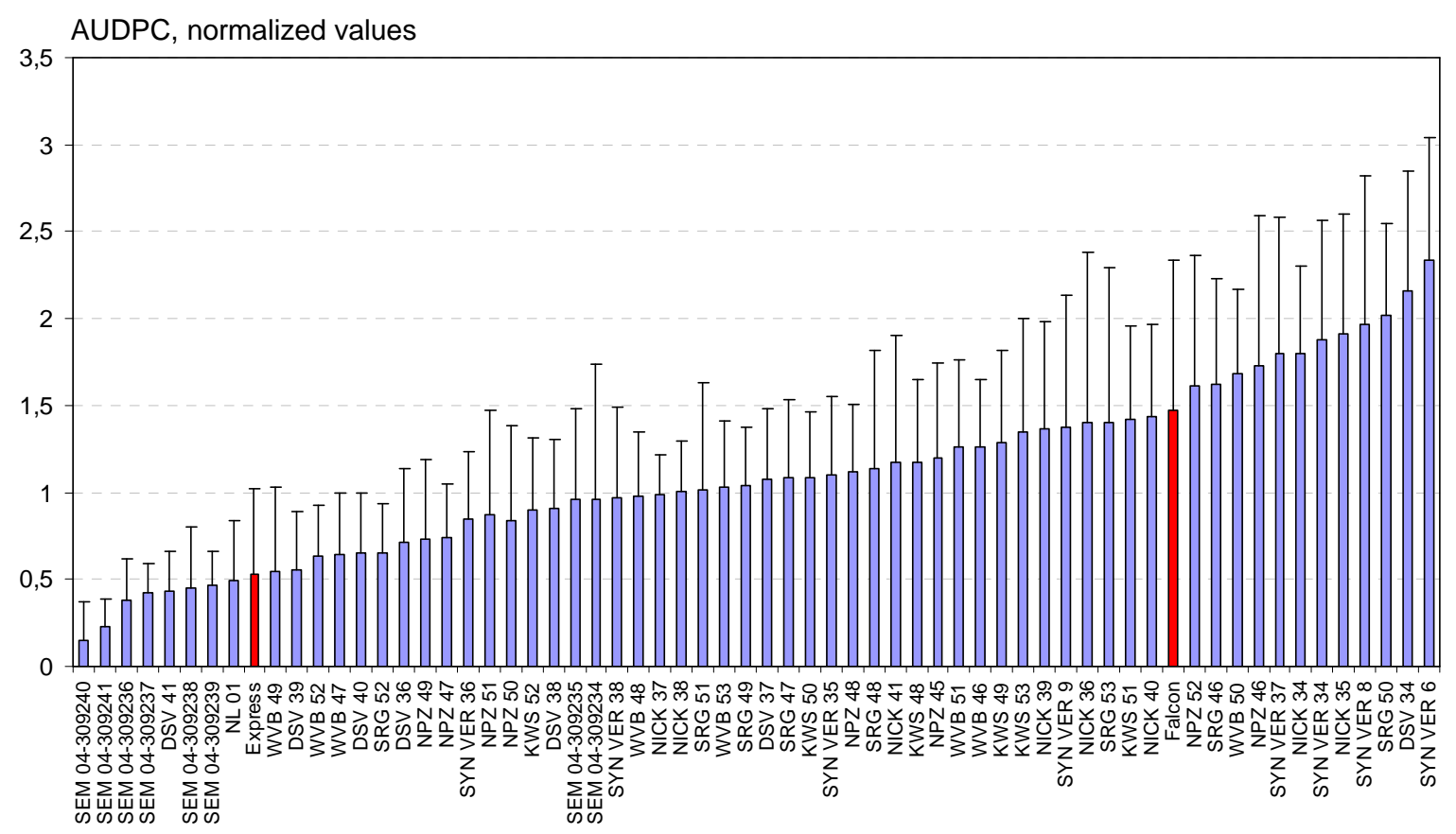

accessions

Fig. A 10. Verticillium longisporum resistance response of B. napus (purple) accessions and reference rapeseed cultivars 'Express' and 'Falcon' (red) measured by area under the disease progress curve (AUDPC) from 17.05.2005 to 07.06.2005. Columns and whiskers represent mean values and standard errors from 20 infected plants of each accession. 


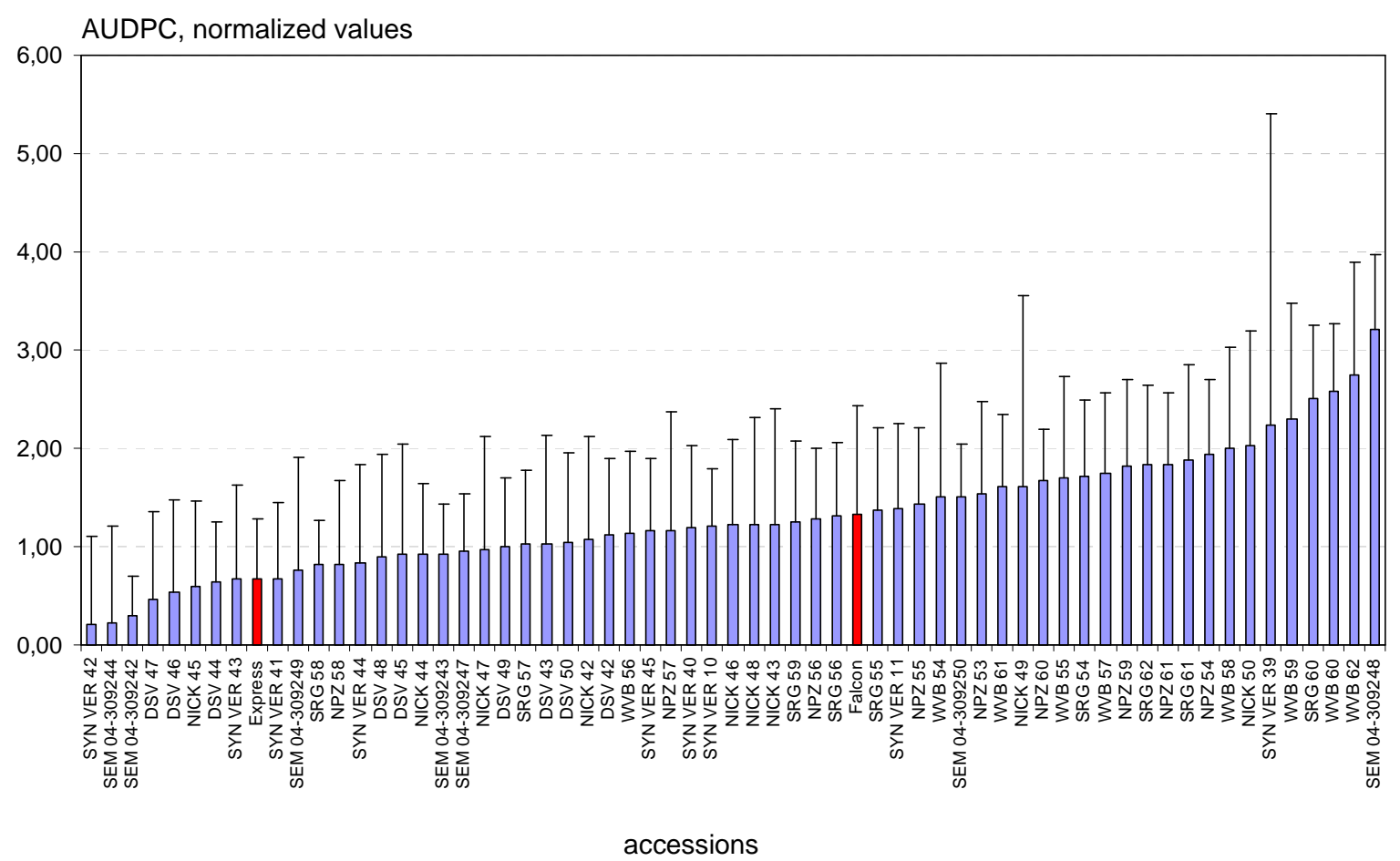

Fig. A 11. Verticillium longisporum resistance response of B. napus (purple) accessions and reference rapeseed cultivars 'Express' and 'Falcon' (red) measured by area under the disease progress curve (AUDPC) from 20.06.2005 to 11.07.2005. Columns and whiskers represent mean values and standard errors from 20 infected plants of each accession.

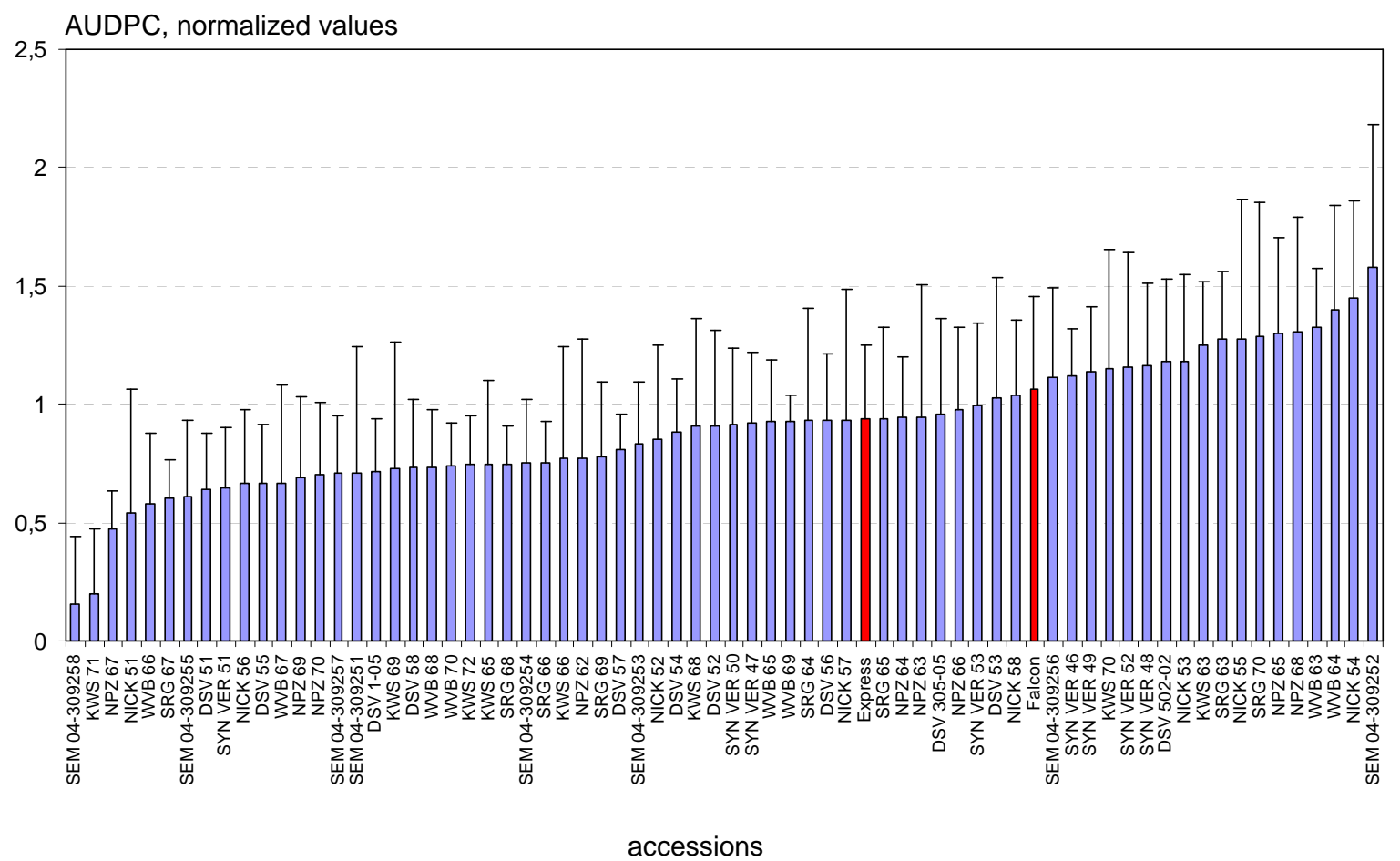

Fig. A 12. Verticillium longisporum resistance response of B. napus (purple) accessions and reference rapeseed cultivars 'Express' and 'Falcon' (red) measured by area under the disease progress curve (AUDPC) from 23.08.2005 to 13.09.2005. Columns and whiskers represent mean values and standard errors from 20 infected plants of each accession. 


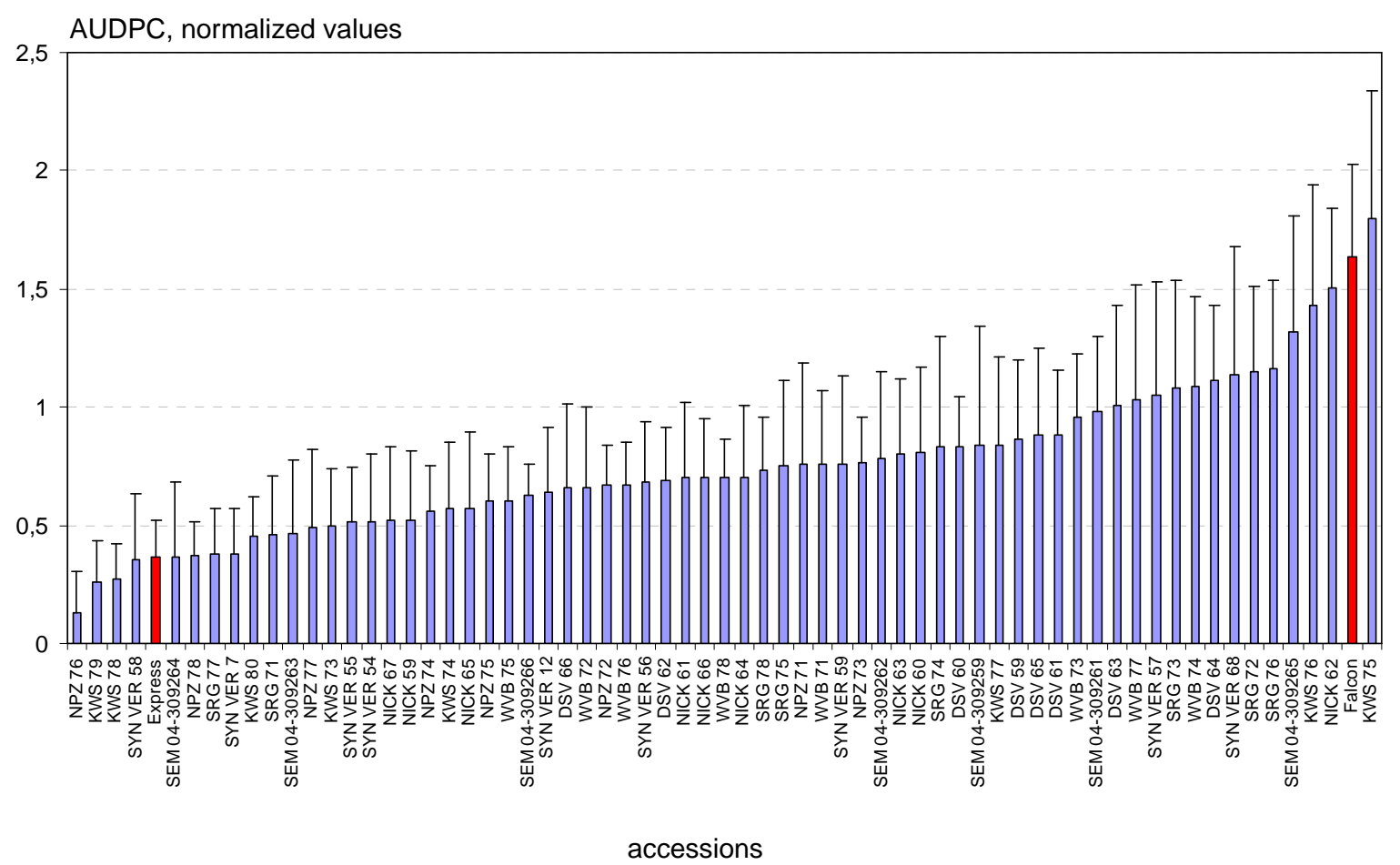

Fig. A 13. Verticillium longisporum resistance response of B. napus (purple) accessions and reference rapeseed cultivars 'Express' and 'Falcon' (red) measured by area under the disease progress curve (AUDPC) from 18.10.2005 to 08.11.2005. Columns and whiskers represent mean values and standard errors from 20 infected plants of each accession.

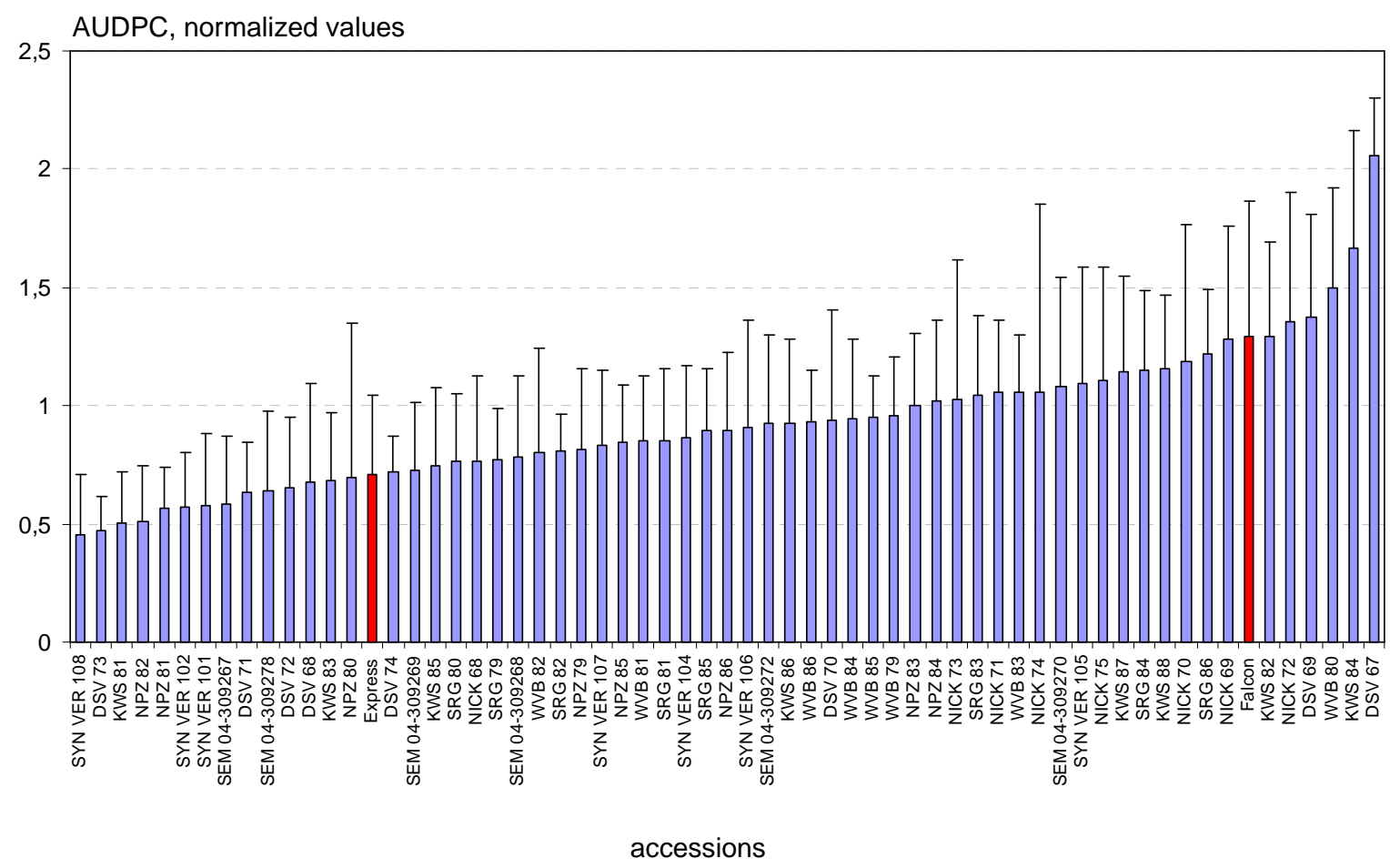

Fig. A 14. Verticillium longisporum resistance response of B. napus (purple) accessions and reference rapeseed cultivars 'Express' and 'Falcon' (red) measured by area under the disease progress curve (AUDPC) from 21.11.2005 to 12.12.2005. Columns and whiskers represent mean values and standard errors from 20 infected plants of each accession. 


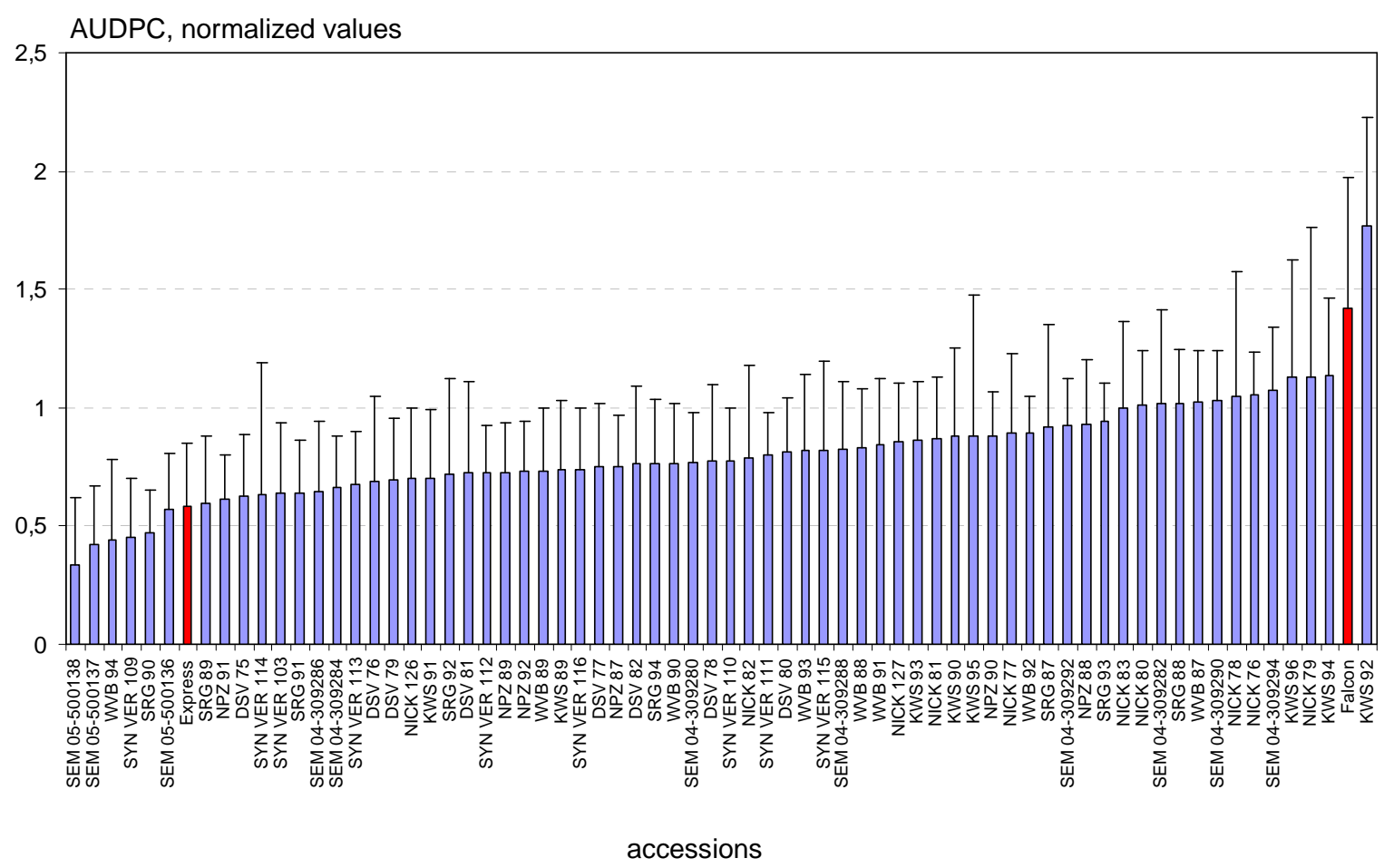

Fig. A 15. Verticillium longisporum resistance response of B. napus (purple) accessions and reference rapeseed cultivars 'Express' and 'Falcon' (red) measured by area under the disease progress curve (AUDPC) from 09.01.2006 to 30.01.2006. Columns and whiskers represent mean values and standard errors from 20 infected plants of each accession.

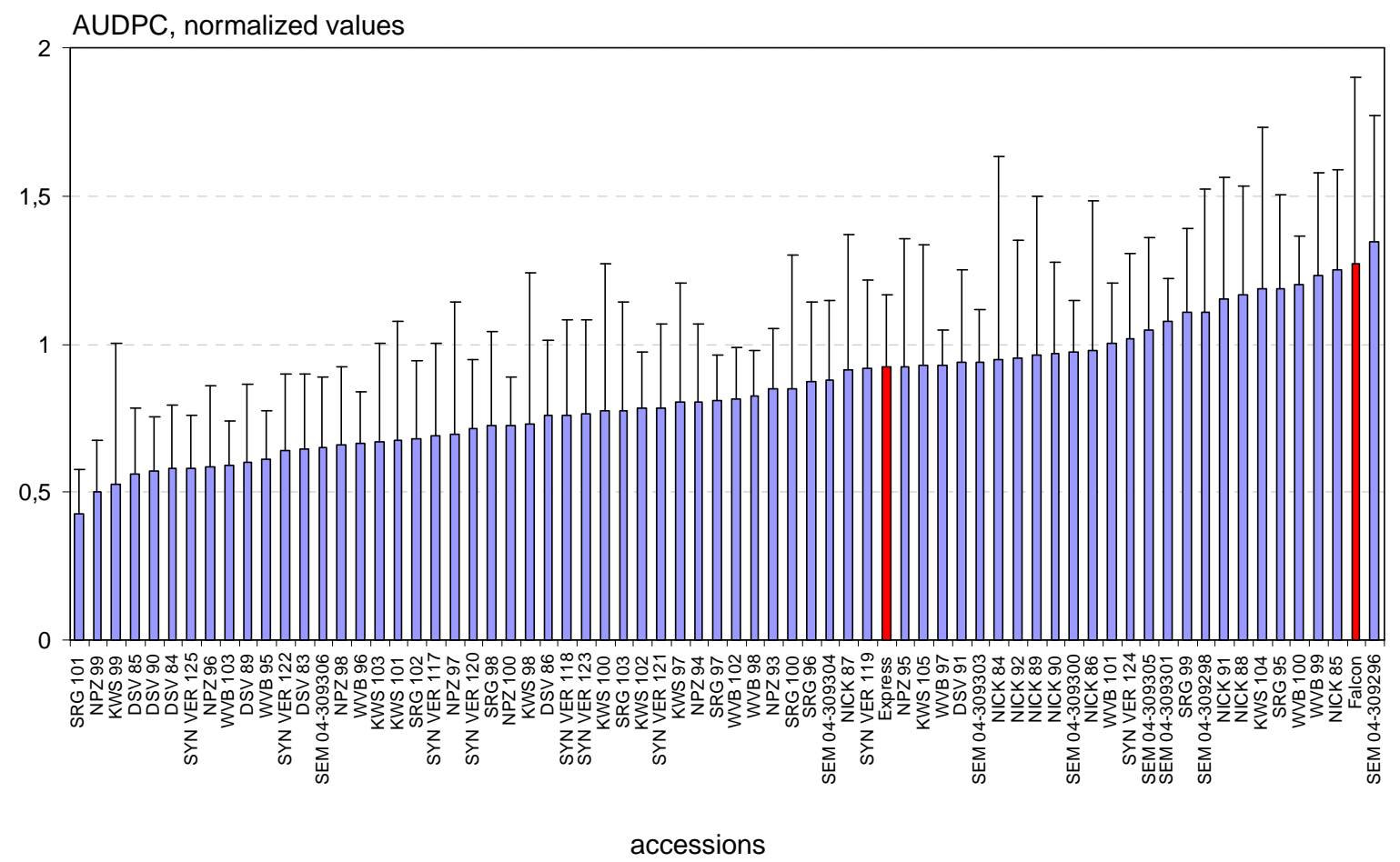

Fig. A 16. Verticillium longisporum resistance response of B. napus (purple) accessions and reference rapeseed cultivars 'Express' and 'Falcon' (red) measured by area under the disease progress curve (AUDPC) from 13.02.2006 to 06.03.2006. Columns and whiskers represent mean values and standard errors from 20 infected plants of each accession. 


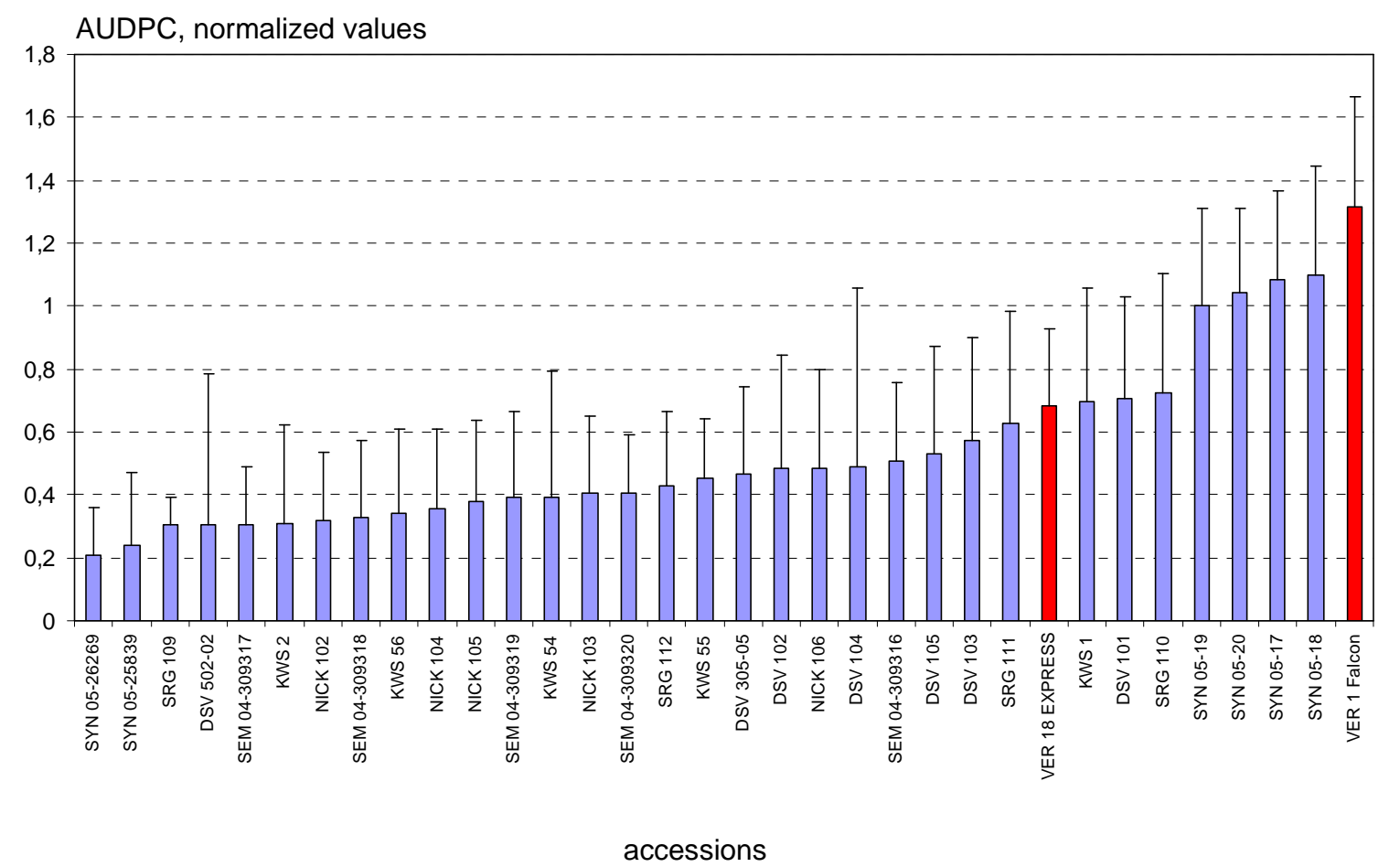

Fig. A 17. Verticillium longisporum resistance response of B. napus (purple) accessions and reference rapeseed cultivars 'Express' and 'Falcon' (red) measured by area under the disease progress curve (AUDPC) from 09.05.2006 to 30.05.2006. Columns and whiskers represent mean values and standard errors from 20 infected plants of each accession.

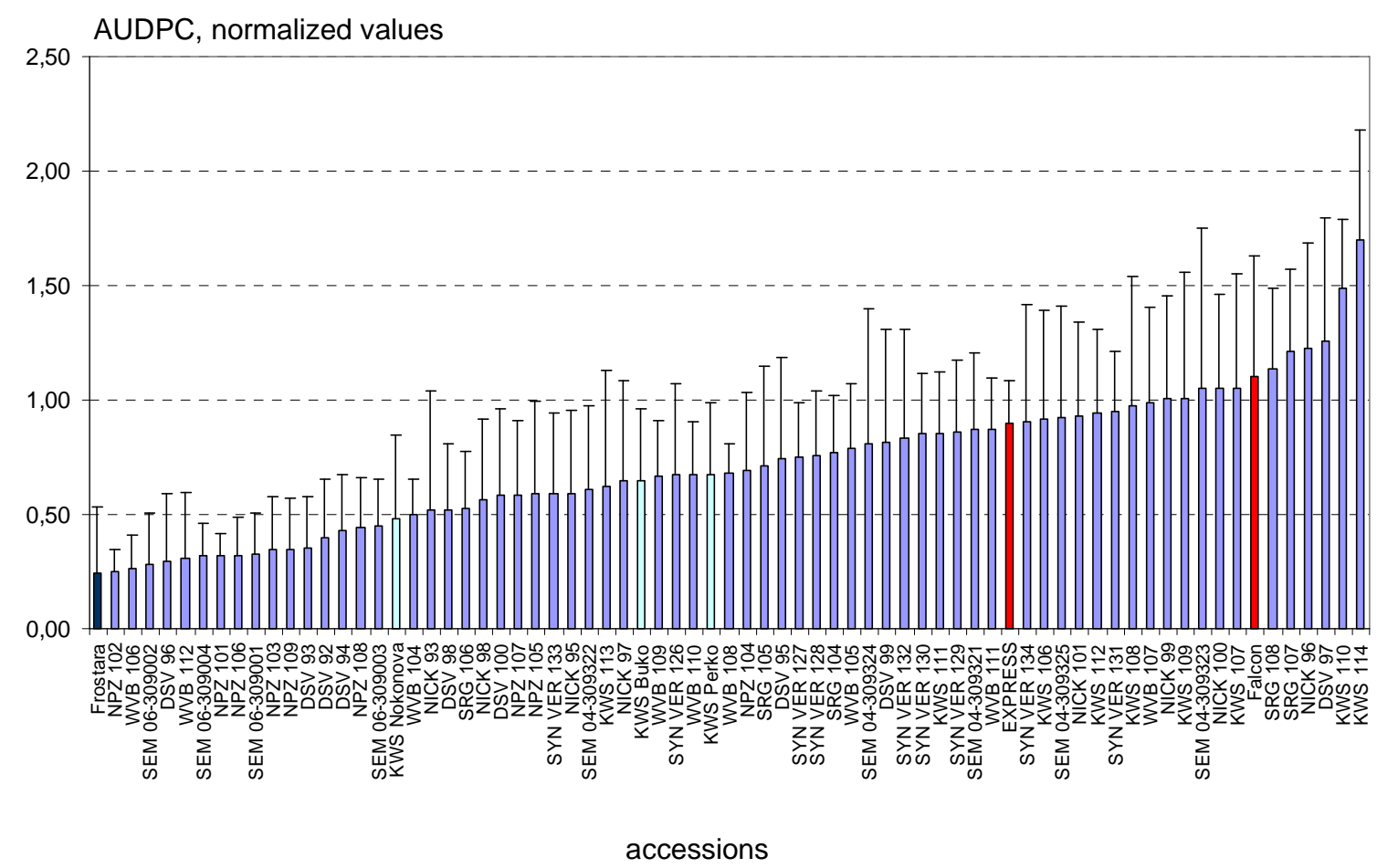

Fig. A 18. Verticillium longisporum resistance response of B. napus (purple), B. oleracea (dark blue) accessions and reference rapeseed cultivars 'Express`and 'Falcon` (red) measured by area under the disease progress curve (AUDPC) from 13.06.2006 to 04.07.2006. Columns and whiskers represent mean values and standard errors from 20 infected plants of each accession. 


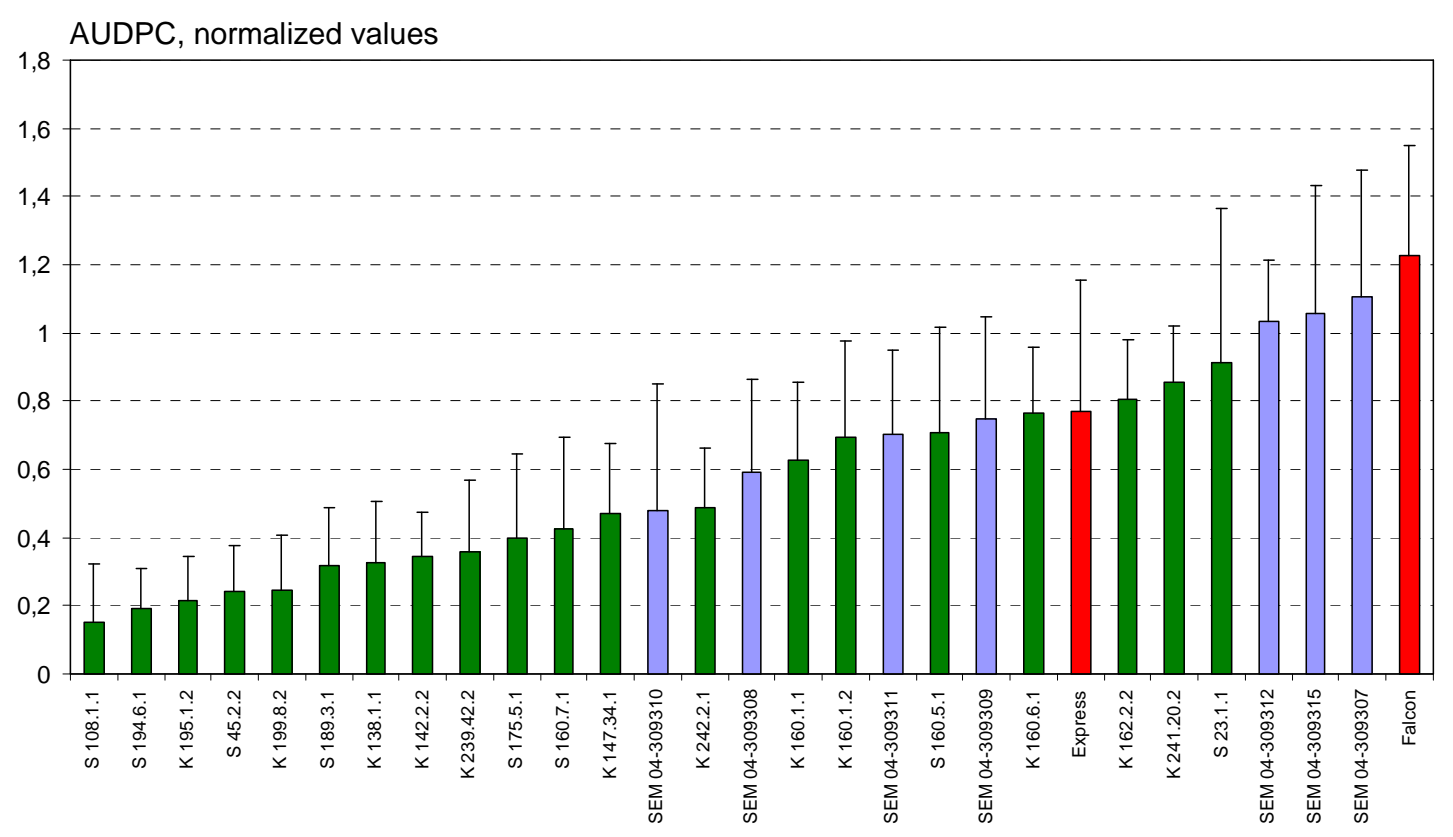

accessions

Fig. A 19. Verticillium longisporum resistance response of B. napus (purple) accessions, resynthesized oilseed rape lines (green) and reference rapeseed cultivars 'Express' and 'Falcon' (red) measured by area under the disease progress curve (AUDPC) from 17.07.2006 to 07.08.2006. Columns and whiskers represent mean values and standard errors from 20 infected plants of each accession.

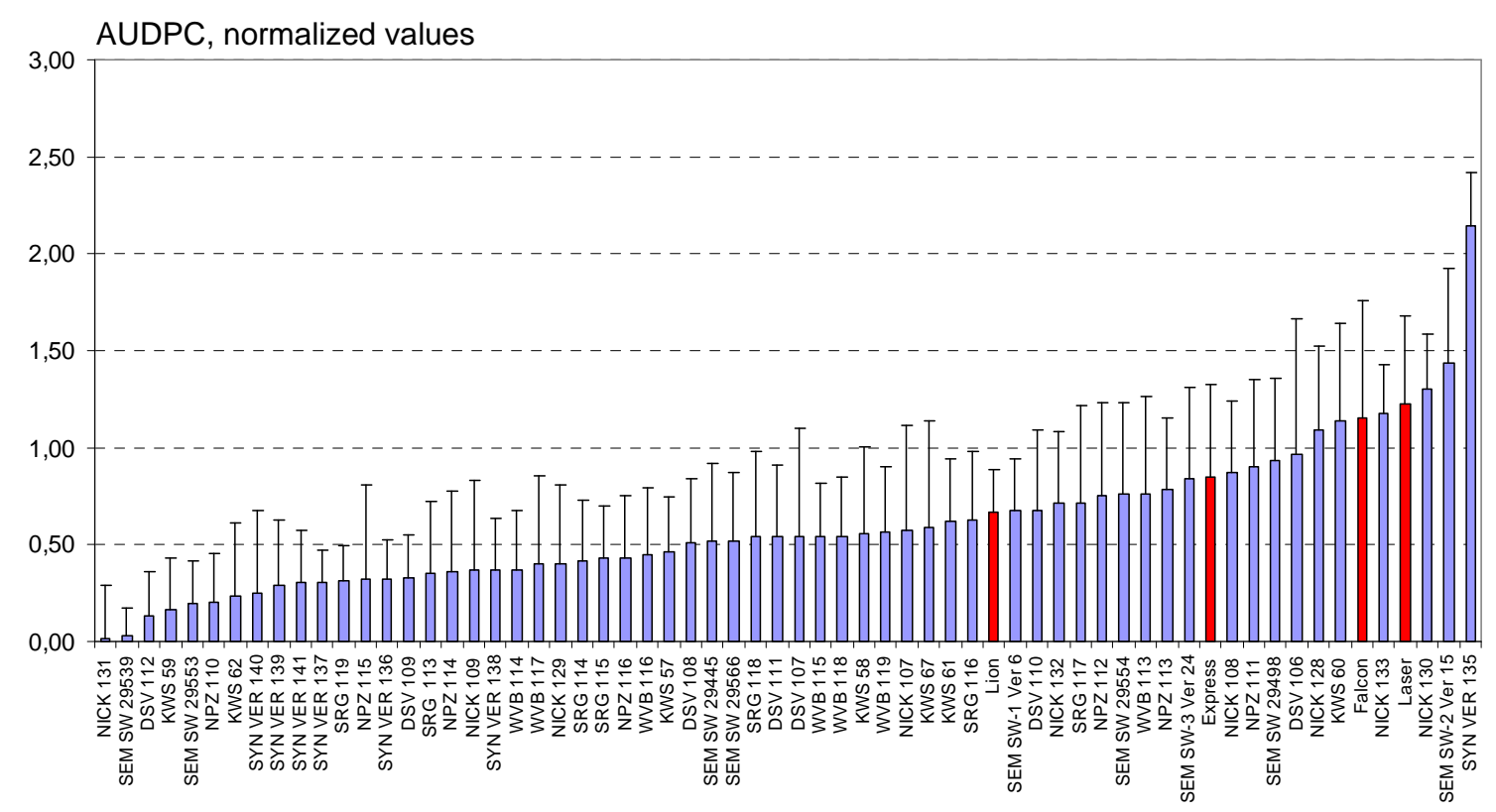

accessions

Fig. A 20. Verticillium longisporum resistance response of B. napus (purple) accessions and reference rapeseed cultivars 'Express' and 'Falcon' (red) measured by area under the disease progress curve (AUDPC) from 27.11.2006 to 18.12.2006. Columns and whiskers represent mean values and standard errors from 20 infected plants of each accession. 


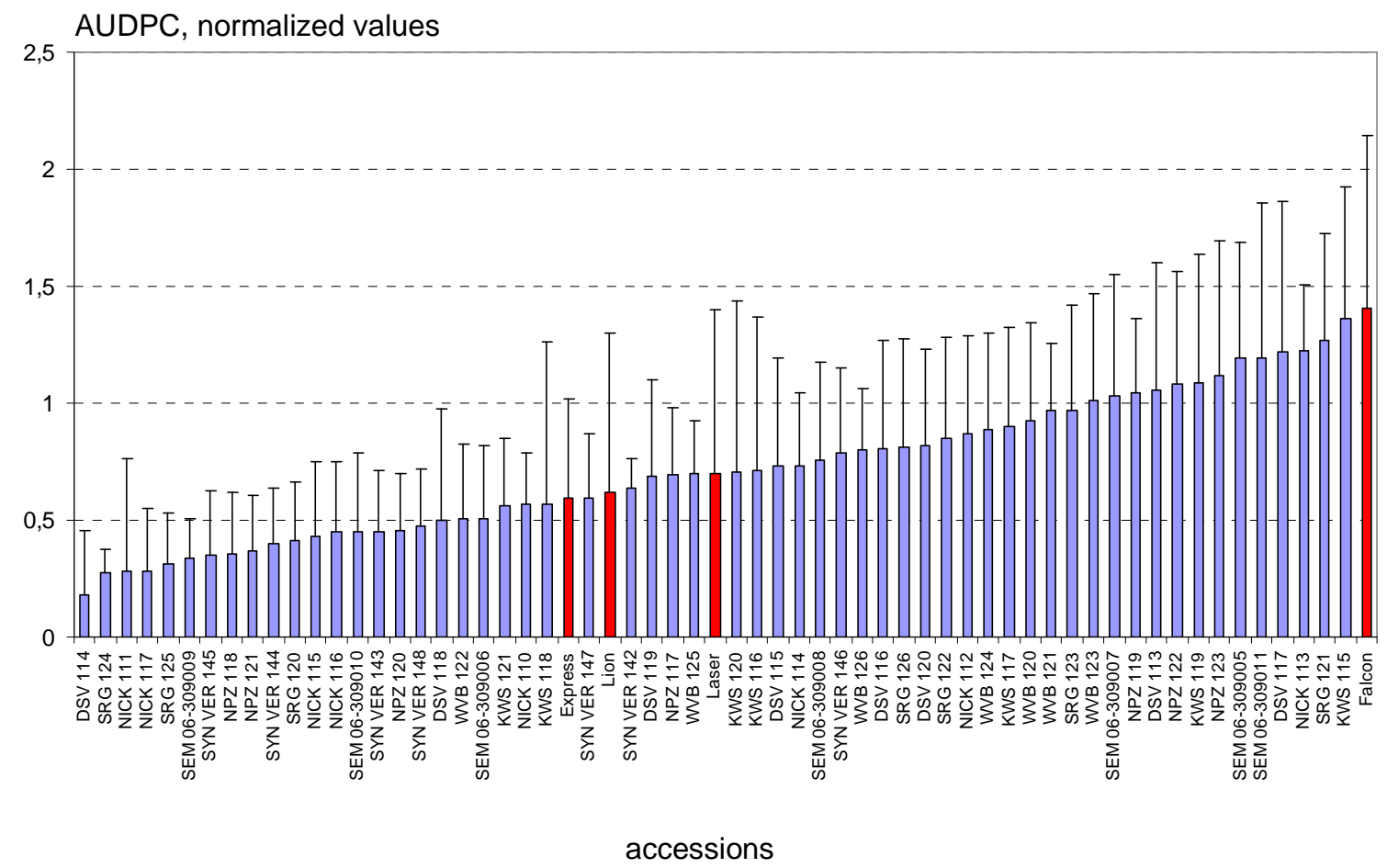

Fig. A 21. Verticillium longisporum resistance response of B. napus (purple) accessions and reference rapeseed cultivars 'Express' and 'Falcon' (red) measured by area under the disease progress curve (AUDPC) from 22.01.2007 to 12.02.2007. Columns and whiskers represent mean values and standard errors from 20 infected plants of each accession.

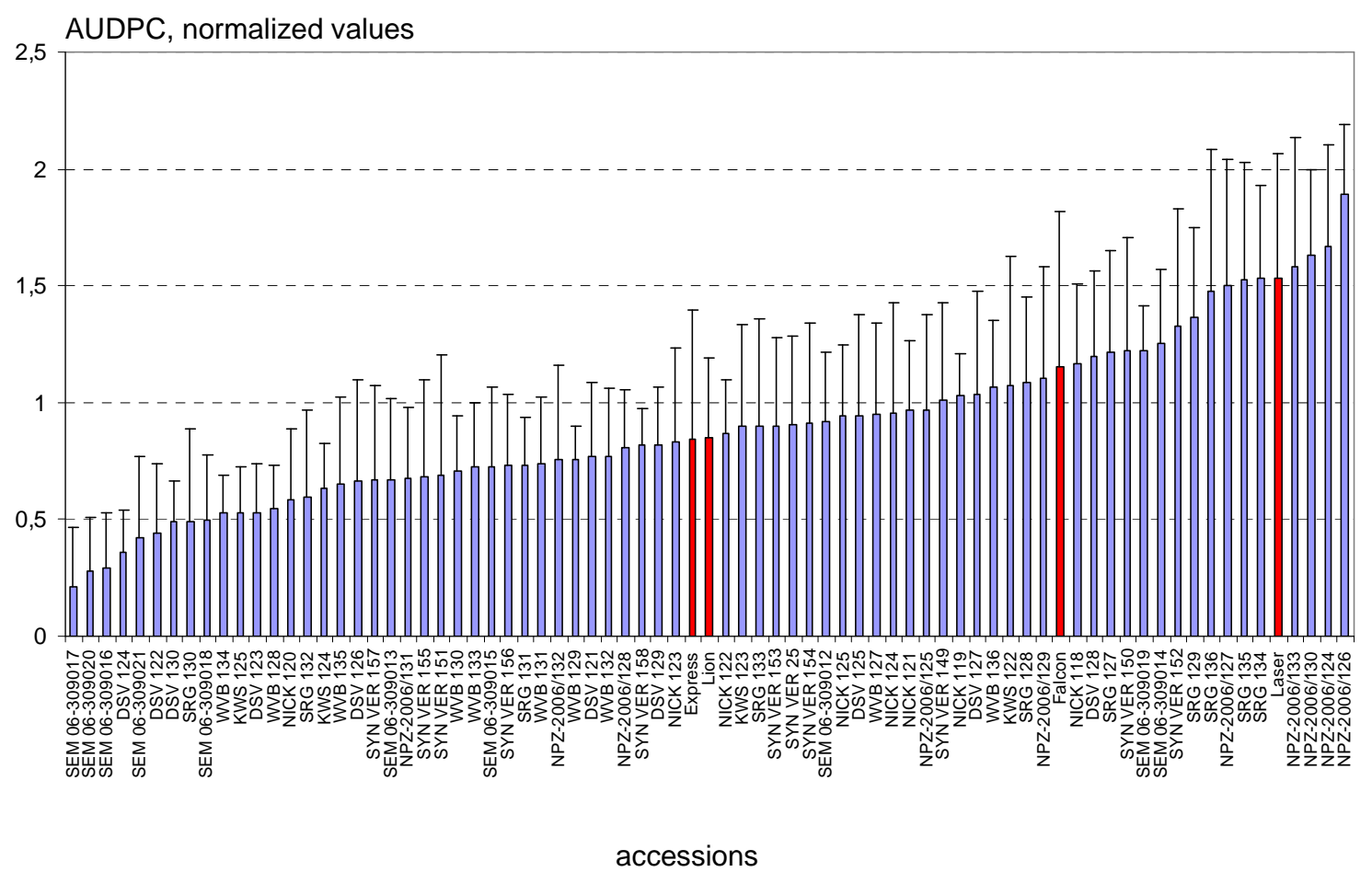

Fig. A 22. Verticillium longisporum resistance response of B. napus (purple) accessions and reference rapeseed cultivars 'Express' and 'Falcon' (red) measured by area under the disease progress curve (AUDPC) from 14.03.2007 to 04.04.2007. Columns and whiskers represent mean values and standard errors from 20 infected plants of each accession. 


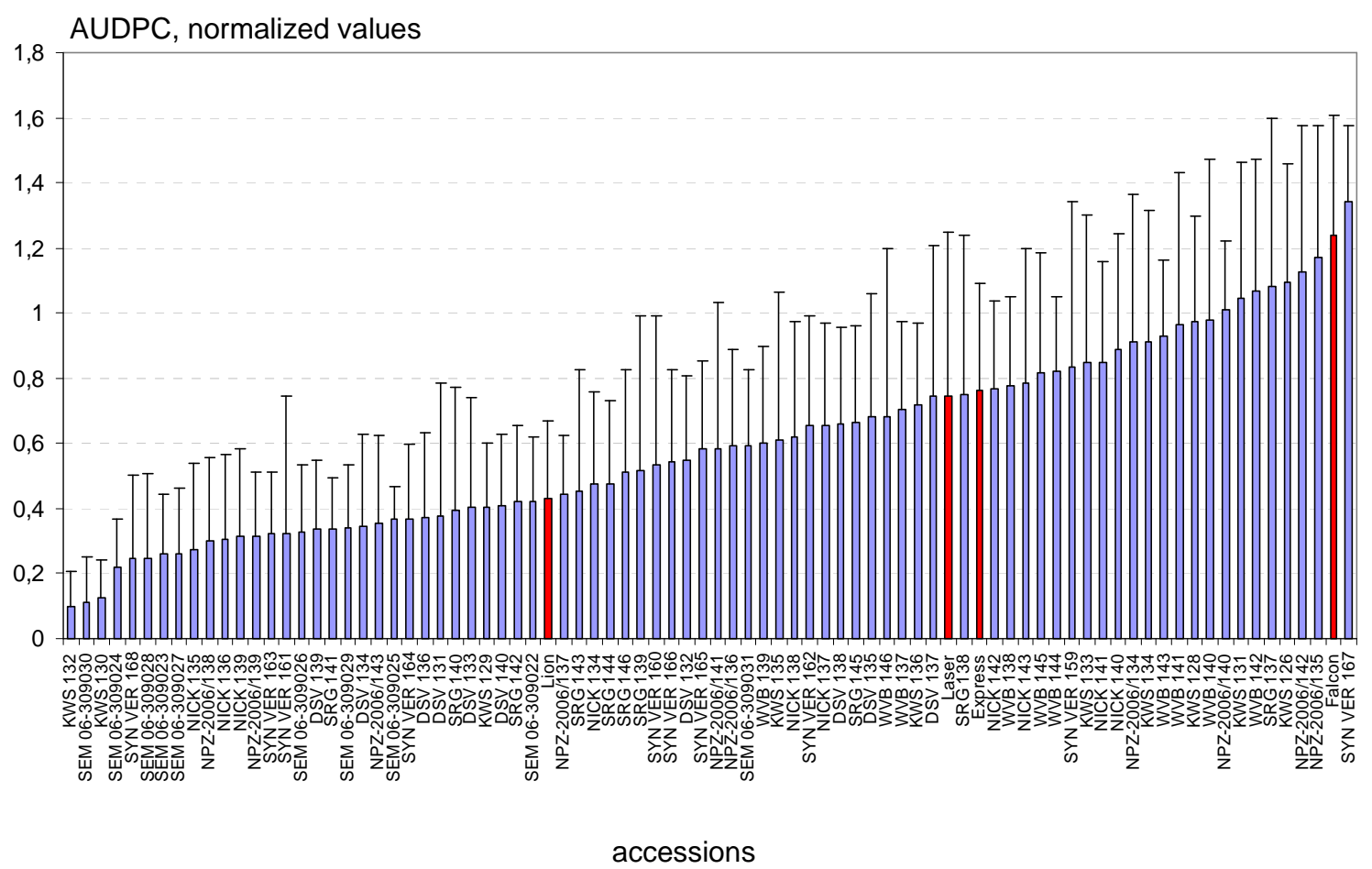

Fig. A 23. Verticillium longisporum resistance response of B. napus (purple) accessions and reference rapeseed cultivars 'Express' and 'Falcon' (red) measured by area under the disease progress curve (AUDPC) from 30.04.2007 to 21.05.2007. Columns and whiskers represent mean values and standard errors from 20 infected plants of each accession.

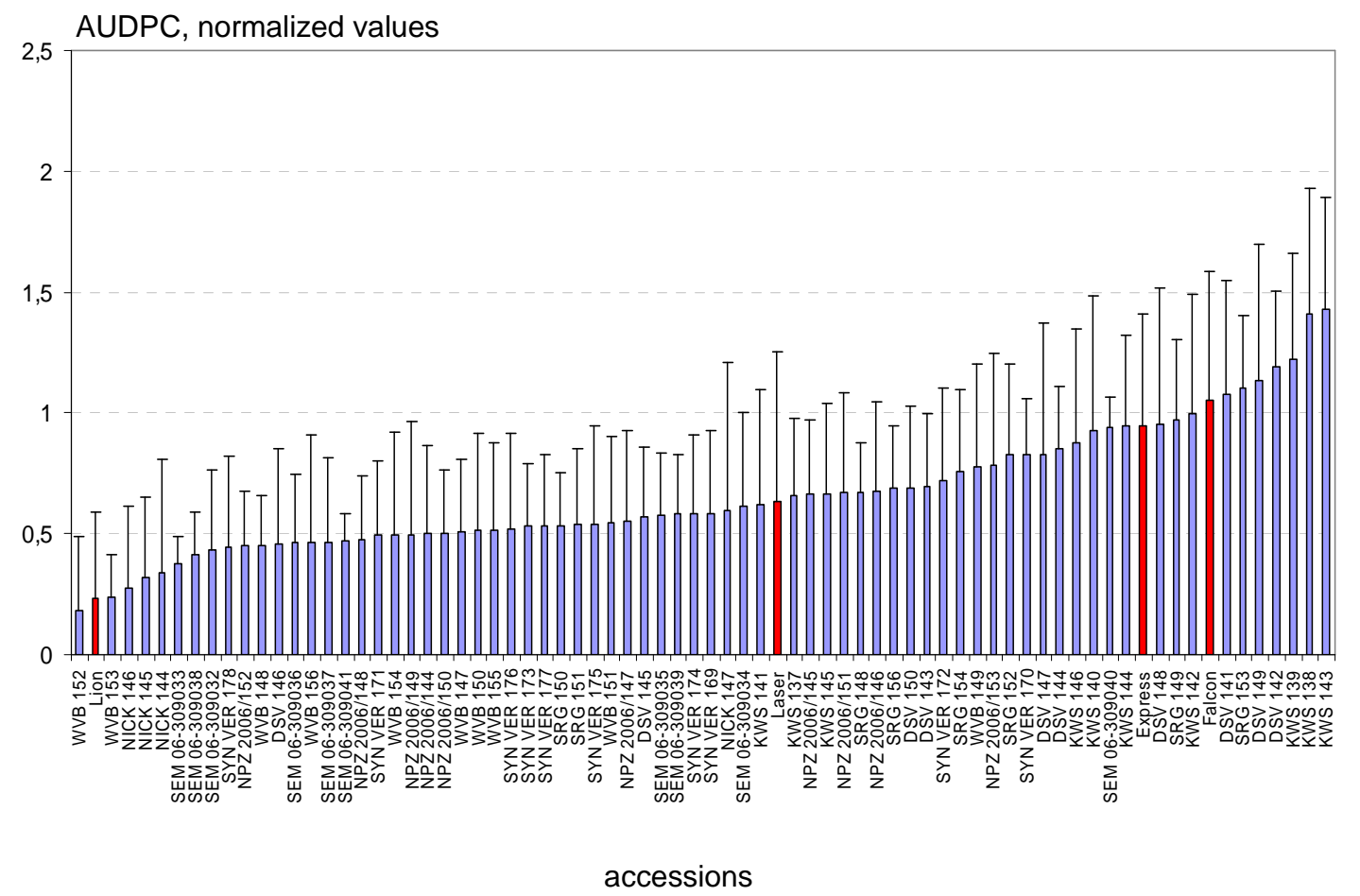

Fig. A 24. Verticillium longisporum resistance response of B. napus (purple) accessions and reference rapeseed cultivars 'Express' and 'Falcon' (red) measured by area under the disease progress curve (AUDPC) from 05.06.2007 to 26.06.2007. Columns and whiskers represent mean values and standard errors from 20 infected plants of each accession. 


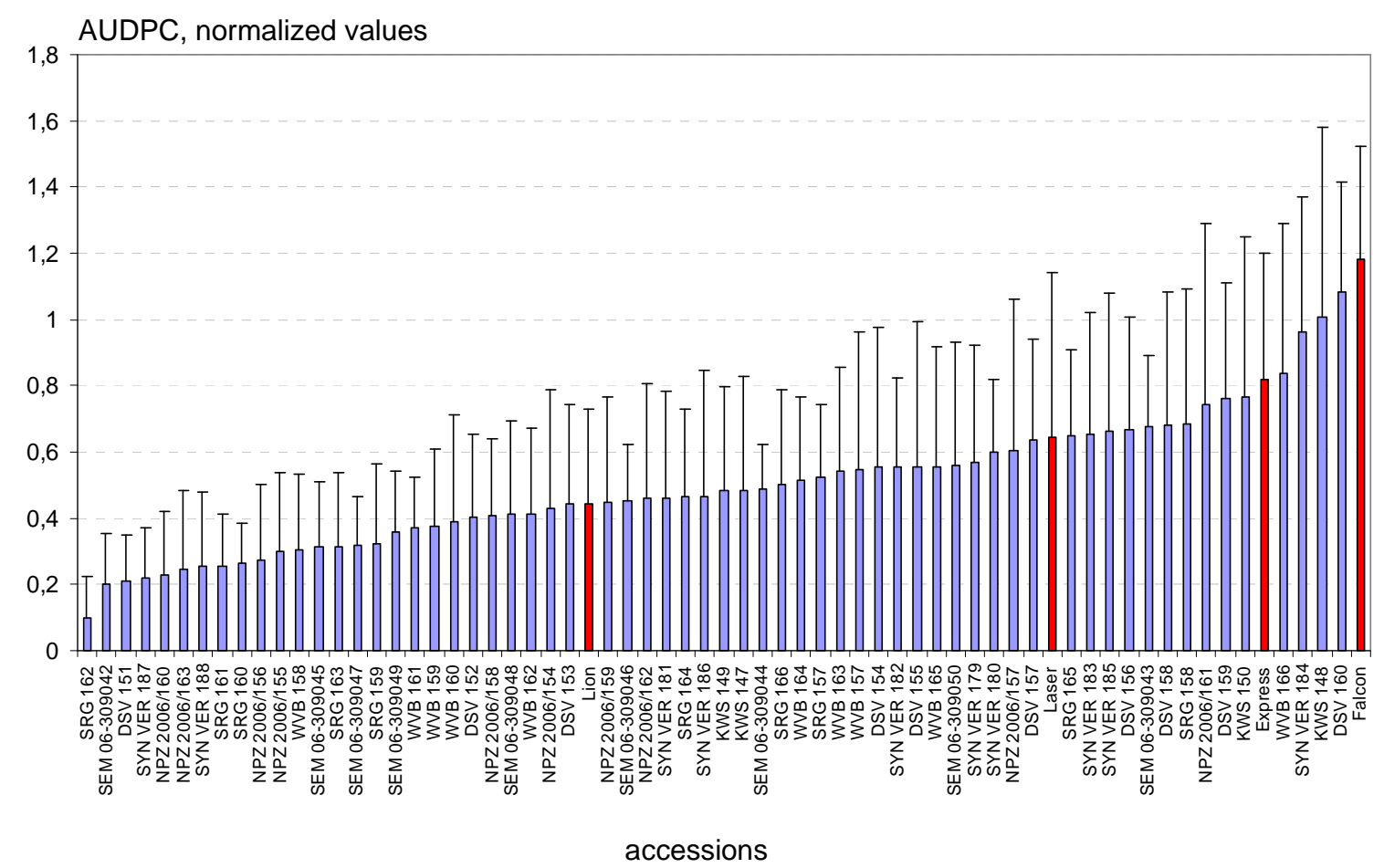

Fig. A 25. Verticillium longisporum resistance response of B. napus (purple) accessions and reference rapeseed cultivars 'Express' and 'Falcon' (red) measured by area under the disease progress curve (AUDPC) from 31.07.2007 to 21.08.2007. Columns and whiskers represent mean values and standard errors from 20 infected plants of each accession. 


\section{Danksagung}

Ich möchte mich ganz besonders bei Herrn Prof. Dr. Andreas von Tiedemann für die Bereitstellung des Themas, die Diskussions- und Hilfsbereitschaft sowie für die gewährte Freiheit und die Unterstützung bei der Durchführung dieser Arbeit und schließlich für die Durchsicht der Manuskripte bedanken.

Bei Herrn Prof. Dr. Heiko Becker bedanke ich mich für die bereitwillige Übernahme des Koreferates.

Birger Koopmann möchte ich für die intensive Betreuung während der Anfangsphase meiner Arbeit, die Einführung in die Molekularbiologie und die stetige Hilfs- und Diskussionsbereitschaft danken.

Bei Andres Schützendübel möchte ich mich für die Unterstützung bei den biochemischen Arbeiten bedanken, seine Diskussionsbereitschaft sowie seinen regen und kritischen Geist, der immer wieder Denkanstöße gibt.

Frau Dr. Gisela Grunewaldt-Stöcker danke ich für die Einführung in die Konfokale Laser Scanning Mikroskopie und die diskussions- und lehrreichen Stunden am CLSM.

Mein herzlicher Dank gilt Jutta Schaper für die Unterstützung im Labor, im Gewächshaus und auf dem Feld, ihre freundliche Art und die Bereitschaft, auch das Wochenende für Probenahmen (und Ausflüge an die Ostsee) zu opfern. Ich bin sehr froh, dass sie in diesem Projekt mitgearbeitet hat.

Bei Evelin Vorbeck möchte ich mich für die herzliche Unterstützung jeglicher Art, das allzeit offene Ohr und für all die Köstlichkeiten bedanken, die sie uns so manches Mal kredenzt hat. Hmmmhh...!

Von ganzem Herzen danken möchte ich Dagmar Tacke für die schöne Zeit in Labor 105 - und nicht nur da -, für die „Zigarettenpausen“ auf der Feuertreppe, das Zuhören und dass sie immer für mich da war.

Bei Nadine Riediger möchte ich mich für das nette Neben- und Miteinander in Labor 163 bedanken. 
Bei Frank Gremmes möchte ich mich dafür bedanken, dass man sich trotz allem immer auf ihn verlassen konnte.

Herrn Hodyl, der mich immer wieder mit seiner dann doch überaus herzlichen und fürsorglichen Art überaschte, gilt mein herzlicher Dank für die exzellente Betreuung unserer Pflanzen im Gewächshaus.

Hubertus Reintke möchte ich für die Hilfe und Unterstützung bei der Durchführung der Feldversuche danken, dafür, dass er so nett über meine Unkenntnisse zur Bestandesführung hinweggesehen hat und für seine freundliche und unkomplizierte Art. Ich habe mich immer auf die Feldtage mit Hubertus und Evi gefreut.

Mein herzlicher Dank gilt auch Martina Bode für die Unterstützung bei allen administrativen Problemen.

Heike Rollwaage, Patricia Bartoschek und Sabine Nutz möchte ich für die Einführung und die Hilfe bei Problemen mit der real-time PCR danken.

Bei Ruth Pilot möchte ich mich für die Durchsicht der Manuskripte bedanken.

Ich möchte mich bei einer ganzen Zahl von Hiwis bedanken, die mir bei der undankbaren Arbeit, Rapsstoppeln zu bonitieren, so sehr geholfen haben: Siv Ahlers, Robert Hodyl, Domingo Uricare, Jorge Saavedra, Anna Kollert, Armin Kollert, Jessica Schramm und Sina Gehlhaar.

Bei der Fachagentur Nachwachsende Rohstoffe (FNR) des Bundesministeriums für Ernährung, Landwirtschaft und Verbraucherschutz (BMELV) und der Gemeinschaft zur Förderung der privaten deutschen Pflanzenzüchtung (GFP e.V.) möchte ich mich für die finanzielle Unterstützung meiner Arbeit bedanken.

Ich bedanke mich bei den am Projektvorhaben beteilgten Züchtern, namentlich Frau Meier zu Beerentrup, Frau Liebenau, Herrn Girke, Herrn Gertz, Herrn Stiewe, Herrn Pleines, Herrn Hemker, Herrn Abel, Herrn Duchscherer, Herrn Hauska und Herrn Horn für die gute 
Zusammenarbeit und die nette Atmosphäre während der alljährlichen Arbeitstreffen in Göttingen und Giessen.

Des Weiteren möchte ich mich bei unseren Projketpartnern von der Pflanzenzüchtung der Universität Giessen für die sehr gute Zusammenarbeit und die netten Arbeitstreffen bedanken. Mein Dank gilt hierbei Herrn Prof. Dr. Wolfgang Friedt, Wilfried Lühs, Rod Snowdon und ganz besonders Wiebke Rygulla; ich hätte keine bessere Projektpartnerin finden können. In Vorfreude auf den gemeinsamen Segeltörn auf der Schlei...

Allen Mitarbeitern des Fachgebietes Pflanzenschutz und Pflanzenpathologie möchte ich für das gute Arbeitsklima und die mir entgegengebrachte Unterstützung meiner Arbeit danken.

Mein tiefer Dank gilt meiner Familie, die an mich geglaubt und mich immer unterstützt hat. 


\section{Publications}

\section{Refereed journals:}

Eynck, C., Koopmann, B., Grunewaldt-Stöcker, G., Karlovsky, P., von Tiedemann, A. (2007) Differential interactions of Verticillium longisporum and $V$. dahliae with Brassica napus detected with molecular and histological techniques. European Journal of Plant Pathology $118,259-274$

Rygulla, W., Snowdon, R.J., Eynck, C., Koopmann, B., von Tiedemann, A., Lühs, W., Friedt, W. (2007a) Broadening the genetic basis of Verticillium longisporum resistance in Brassica napus by interspecific hybridisation. Plant Breeding, Online Early Articles

Rygulla, W., Seyis, F., Lühs, W., Eynck, C., von Tiedemann, A., Friedt, W, Snowdon, R.J. (2007b) Combination of resistance to Verticillium longisporum from zero erucic acid Brassica oleracea and oilseed Brassica rapa genotypes in resynthesized rapeseed (Brassica napus) lines. Phytopathology 97 (11), 1391-1396

\section{Conference Proceedings:}

Eynck, C., Grunewaldt-Stöcker, G., Koopmann, B., von Tiedemann, A. (2007) Verticillium longisporum - identifying resistance to a new threat to winter oilseed rape. Proceedings of the 12th International Rapeseed Congress (IV).

Eynck, C., Grunewaldt-Stöcker, G., Koopmann, B., von Tiedemann, A. (2006) Comparative histological studies on the interaction of Verticillium longisporum and $V$. dahliae with roots of Brassica napus. IOBC/wprs Bulletin Vol. 29 (7): 371-377 


\section{Curriculum Vitae}

\section{Personal data}

name:

date of birth:

place of birth:

family status:

place of residence:

phone:

e-mail:
Christina Eynck

17.01.1978

Bad Karlshafen

unmarried

Föhrenwg 4, 37077 Göttingen

0551/250 4943

ceynck@gwd.de

\section{Secondary education}

09/1984 until 07/1988

primary school, Oster-Ohrstedt

08/1988 until 06/1997

grammar school (Theodor-Storm-Schule), Husum

graduation:

high school diploma

\section{Tertiary education}

from $10 / 1997$

04/1998 until 07/1999

10/1999

$06 / 2002$

$11 / 2002$

08/2002 until 05/2003

from $01 / 2004$
Christian-Albrechts-Universität zu Kiel, studies of biology and agricultural sciences, specialization plant production intermediate diploma agricultural sciences intermediate diploma biology Bachelor of Science agricultural sciences Master of Science agricultural sciences trainee fellowship MPIZ Köln PhD student at Department of Crop Sciences, Section Plant Pathology and Plant Protection, University of Göttingen 


\section{Presentations at national and international conferences:}

Eynck, C., von Tiedemann, A. (2007) Resistenzfaktoren in Raps gegen das vaskuläre Pathogen $V$. longisporum. Oral presentation on the Annual Meeting for Resistance in Crop Plants. Fulda, Germany, 10./11. 12.2007

Eynck, C., Grunewaldt-Stöcker, G., Koopmann, B., von Tiedemann, A. (2007) Verticillium longisporum - identifying resistance to a new threat to winter oilseed rape. Oral presentation on $12^{\text {th }}$ International Rapeseed Congress. Wuhan, China, 26.-30.03.2007

Eynck, C., von Tiedemann, A. (2006) Screening for new sources of resistance and identification of resistance factors against Verticillium wilt on oilseed rape. Oral presentation on the Annual Meeting of the Association of German Plant Breeders (GFP). Bonn, Germany, 08.11.2006

Eynck, C., Grunewaldt-Stöcker, G., Koopmann, B., von Tiedemann, A. (2006) Infection process and spread of Verticillium longisporum on oilseed rape. Oral presentation on $55^{\text {th }}$ German Congress of Plant Protection. Goettingen, Germany, 25.-28.09.2006

Eynck, C., Grunewaldt-Stöcker, G., Koopmann, B., von Tiedemann, A. (2006) Host- and non-host interaction of $V$. longisporum and $V$. dahliae with $B$. napus. Oral presentation on Work Shop „Mycology“ of the German Phytopathological Society (DPG). Berlin, Germany, 16./17.03.2006

Eynck, C., von Tiedemann, A. (2005) Screening for new sources of resistance and identification of resistance factors against Verticillium wilt on oilseed rape. Oral presentation on the Annual Meeting of the Association of German Plant Breeders (GFP). Bonn, Germany, 03.11.2005

Eynck, C., Grunewaldt-Stöcker, G., Koopmann, B., von Tiedemann, A. (2005) Comparative histological studies on the interaction of Verticillium longisporum and $V$. dahliae with roots of Brassica napus. Oral presentation on IOBC Working Group Meeting. Poznan, Poland, 11./12.10.2005

Eynck, C., Koopmann, B., Grunewaldt-Stöcker, G., von Tiedemann, A. (2005) Interaction of Verticillium longisporum and Verticillium dahliae with oilseed rape - root infection and systemic spread. Oral presentation on Verticillium Symposium, California, USA, 19.06.2005

Eynck, C., Koopmann, B., von Tiedemann, A. (2005) Investigations on the interaction of Verticillium spp. on roots of Brassica napus and Linum usitatissimum using GFP-tagged strains of $V$. longisporum and $V$. dahliae. Oral presentation on Work Shop "Plant-microbeinteractions" of the German Phytopathological Society (DPG). Freiburg, Germany, 17.03.2005

Eynck, C., von Tiedemann, A. (2004) Screening for new sources of resistance and identification of resistance factors against Verticillium wilt on oilseed rape. Oral presentation on the Annual Meeting of the Association of German Plant Breeders (GFP). Bonn, Germany, 03.11.2004

Rygulla, W., Eynck, C., Koopmann, B., von Tiedemann, A., Lühs, W., Snowdon, R., Friedt, W. (2004) Resynthesised Brassica napus as a genetic resource for improvement of Verticillium wilt resistance in oilseed rape. Poster presentation on the XVIIth EUCARPIA General Congress, Tulln, Austria, 08.-11-09.2004 


\section{Eidestattliche Erklärung}

Hiermit erkläre ich eidestattlich, dass dises Dissertation selbständig und ohne unerlaubte Hilfe angefertigt wurde.

Göttingen, im Dezember 2007 